


\title{
Pfadabhängigkeit strategischer Entscheidungen
}

\author{
Eine Fallstudie am Beispiel des
}

\section{Bertelsmann Buchclubs Deutschland}

Inaugural-Dissertation zur Erlangung des akademischen Grades eines Doktors der Wirtschaftswissenschaft (Dr. rer. pol.)

des Fachbereichs Wirtschaftswissenschaft der Freien Universität Berlin

\author{
vorgelegt von: $\quad$ Dipl.-Kfm. Jan Philip Holtmann \\ Anschrift: $\quad$ Fregestraße 70, 12159 Berlin \\ Telefon: $\quad 017620037660$
}

Berlin, Juni 2008
1. Gutachter:
Prof. Dr. Georg Schreyögg
2. Gutachterin:
PD Dr. habil. Michaela Haase 
Dekan:

Erstgutachter:

Zweitgutachterin:

Tag der Disputation:
Prof. Dr. Jörg Sydow

Prof. Dr. Georg Schreyögg

PD Dr. habil. Michaela Haase

16. Juli 2008 

Jan Philip Holtmann

\section{Pfadabhängigkeit strategischer Entscheidungen}

Eine Fallstudie am Beispiel des Bertelsmann Buchclubs Deutschland

Mit einem Geleitwort von

Prof. Dr. Georg Schreyögg 


\section{Bibliografische Information Der Deutschen Bibliothek}

Die Deutsche Bibliothek verzeichnet diese Publikation in der Deutschen Nationalbibliografie; detaillierte bibliografische Daten sind im Internet über http://dnb.ddb.de abrufbar.

Dissertation D 188 (Diss. Freie Universität Berlin, 2008).

Alle Rechte vorbehalten

(C) Jan Philip Holtmann und

Kölner Wissenschaftsverlag

Albers, Peters \& Reihlen GbR

Köln, 2008

http://www.primescience.com

http://www.koelnerwissenschaftsverlag.de

Das Werk einschließlich seiner Teile ist urheberrechtlich geschützt. Jede Verwertung außerhalb der engen Grenzen des Urheberrechtsgesetzes ist ohne Zustimmung des Verlages unzulässig und strafbar. Das gilt insbesondere für Vervielfältigungen, Übersetzungen, Mikroverfilmungen und die Einspeicherung und Verarbeitung in elektronischen Systemen.

Die Wiedergabe von Gebrauchsnamen, Handelsnamen, Warenbezeichnungen, usw. in diesem Werk berechtigt auch ohne besondere Kennzeichnung nicht zu der Annahme, dass solche Namen im Sinne der Warenzeichen- und Markenschutz-Gesetzgebung als frei zu betrachten wären und daher von jedermann benutzt werden dürfen.

Umschlaggestaltung: Susanne Klee

Printed in Germany

ISBN: 978-3-937404-57-8 


\section{Geleitwort}

In der Betriebswirtschaftslehre wurde strategische Entscheidungsforschung lange Zeit primär aus einer präskriptiven Perspektive betrieben. Man strebte danach, optimale Entscheidungen zu bestimmen und falsche oder suboptimale Entscheidungen als solche zu markieren.

Deskriptive Elemente im Sinne einer empirischen Entscheidungstheorie fanden in diese Perspektive nur mühsam Eingang. Neben informatorischen Beschränkungen in Form von „bounded rationality“ waren es vor allem potenzielle Interessengegensätze zwischen „Prinzipalen“ und „Agenten“, die sich Gehör verschaffen konnten. In den letzten Jahren machen ganz andere Studien auf sich aufmerksam, die faktische Entscheidungsverläufe in Organisationen und ihre Bestimmungsgründe vor dem Hintergrund systemischer Dynamiken und sich selbst verstärkender Prozesse analysieren. Ein besonderes Interesse findet dabei die Theorie der Pfadabhängigkeit, in der Rigiditäten oder Beharrungskräfte in den Vordergrund gerückt werden, die Organisationen daran hindern, einmal getroffene Entscheidungen zu verändern oder neue Vorhaben aufzunehmen.

Die Pfad-Perspektive stellt das Prozessgeschehen und nicht mehr die Einzelentscheidung in das Zentrum der Analyse, die Eigenart des Prozessgeschehens bestimmt das Prozessergebnis und seine Irreversibilität. Die Logik des Prozessgeschehens bedarf einer genauen Analyse.

Die Erklärung von Rigiditäten und Verriegelungen („lock-ins“) durch Pfadabhängigkeit ist noch ein relativ junges Forschungsfeld. Nicht jede Rigidität kann als Ausdruck von Pfadabhängigkeit interpretiert werden, hier ist gleich zu Beginn Präzision erforderlich, sonst verfließt das Konzept der Pfadabhängigkeit ins Unbestimmte. In genau dieses Bemühen ist auch vorliegende Studie einzuordnen. Auf der Basis einer präzisen Fassung der Theorie der Pfadabhängigkeit analysiert der Autor Entscheidungspro- 
zesse bei dem Bertelsmann Buchclub Deutschland, um eine Erklärung für das Festhalten an dem Buchclub-Geschäftsmodell trotz jahrelanger Verluste zu gewinnen.

Um einen Einblick in das Wirkungsgeschehen zu gewinnen, wird eine Intensivfallstudie durchgeführt. Dabei legt der Autor von vorne herein klar, dass mit einer solchen Einzelfallstudie eine Generalisierung auf eine ganze Population nicht möglich ist, aber auch gar nicht angestrebt wird. Es ist nicht seine Absicht zu behaupten, dass alle Buchclubs pfadabhängig sind, zu zeigen ist vielmehr, dass bei Vorliegen der spezifizierten Voraussetzungen auch tatsächlich Pfadabhängigkeit eintritt.

Die Arbeit ist insgesamt sehr stringent angelegt; die Argumentation ist sauber strukturiert und folgt einem schlüssigen Konzept. Besonders hervorzuheben ist das Bestreben des Verfassers, den pfadtheoretischen Analyserahmen bei der Interpretation der empirischen Beobachtungen und des Fallgeschehens stets als Bezugsrahmen zu verwenden. Die strenge Einhaltung dieses Prinzips ist ein Qualitätsmerkmal vorliegender Arbeit.

Der Verfasser hat mit sehr viel Engagement und Eigenständigkeit ein neues Gebiet wissenschaftlich bearbeitet und erste empirische Ergebnisse zu einem bislang nur wenig untersuchten Gegenstand vorgelegt. Es zeichnet sich bereits jetzt ab, dass die Arbeit geeignet ist, die Fachdiskussion fundiert zu bereichern.

Ich wünsche dieser innovativen Arbeit weite Verbreitung und das hohe Maß an Aufmerksamkeit, das ihr gebührt. 


\section{Vorwort}

Eine Entscheidung liegt dann vor, wenn eine von mehreren, sich gegenseitig ausschließenden Handlungsmöglichkeiten ausgewählt wird. Die Entscheidung für eine Handlungsmöglichkeit impliziert somit zugleich den Verzicht auf Alternativen. Diese Definition des Entscheidungsbegriffs ist theoretische Grundlage für die vorliegende Dissertationsschrift - und trifft in erstaunlicher Klarheit auf die Entscheidung zu deren Erstellung zu: Auch Promovieren heißt verzichten!

Dass mir dieser Verzicht alles andere als schwer gefallen ist und zugleich eine fachliche wie persönliche Bereicherung für mich bedeutete, verdanke ich einer ganzen Reihe von Menschen, die mich während meiner Zeit an der Freien Universität Berlin ganz nah oder aus der Ferne unterstützt haben.

Allen voran gebührt mein Dank meinem dreiköpfigen Betreuungsteam - Herrn Prof. Dr. Georg Schreyögg, Frau PD Dr. Michaela Haase sowie Herrn Dr. Jochen Koch -, das von Beginn an meinen Ideen offen und interessiert gegenüberstand und sich stets Zeit für Diskussionen und wissenschaftlichen Austausch nahm. Doktorandenseminare, Vortragsreihen und die permanente Möglichkeit zum persönlichen Diskurs - all diese Mosaiksteine trugen auf ihre Art und Weise zum Fortschritt, aber auch zur kritischen Auseinandersetzung mit Theorie, Methodik und Empirie, bei.

Eine empirische Arbeit ist nicht ohne umfangreiches Datenmaterial zu erstellen. Aus diesem Grund möchte ich mich ausdrücklich bei den zahlreichen ehemaligen und derzeitigen Mitarbeitern der Bertelsmann AG und Branchenkennern des Buchmarkts für Interviewtermine, Gesprächsbereitschaft und ausführliche Recherchemöglichkeiten bedanken.

Einen mindestens ebenso großen Beitrag zum Gelingen dieser Arbeit lieferte mein soziales Umfeld - allen Voran meine 15 Doktoranden-Kollegen, mit denen ich nicht nur den Arbeitsplatz teilte, sondern aus denen im Laufe der Zeit über den fachlichen 
Diskurs hinaus private Freundschaften entstanden sind. Besonders bedanken möchte ich mich an dieser Stelle bei Anja Mante, Marc Rödenbeck, Jan Strobel, John Lüttel sowie Sebastian Botzem, ohne deren fachliche sowie menschliche Unterstützung die Arbeit in dieser Form sicherlich nicht möglich gewesen wäre.

Den immer wieder notwendigen Abstand zur Wissenschaft, die Vermeidung meines persönlichen Lock-Ins, verdanke ich meinen Freunden und Wegbegleitern Didi, Lars, Sille, Verena, Nina, Anne, Juraj, Zirn, Tina, Jerôme, Enrique, Christian, Markus, der Bielefelder WG und Stephan, der wohl als damaliger Assistent am Lehrstuhl für Organisation an der Universität Bayreuth - um in der Sprache der Pfadabhängigkeit zu bleiben - das auslösende Small Event für meine akademische Laufbahn bedeutete. Euch allen vielen Dank!

Schließlich gilt mein besonderer Dank meiner Familie, die mich während meiner Promotion permanent tatkräftig unterstützte und immer an mich geglaubt hat. Euch ist diese Arbeit gewidmet.

Diese Arbeit wurde im Juli 2008 vom Fachbereich Wirtschaftswissenschaft der Freien Universität Berlin als Dissertation angenommen. Dabei wurde ich als Stipendiat der Deutschen Forschungsgemeinschaft unterstützt, der mein herzlicher Dank gilt. Für die Drucklegung wurden lediglich redaktionelle Änderungen vorgenommen. 


\section{Inhaltsverzeichnis}

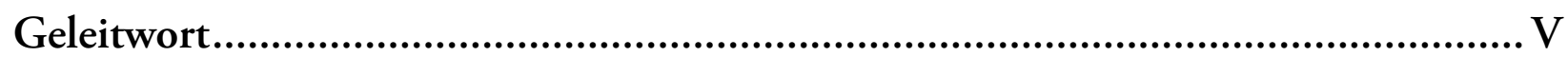

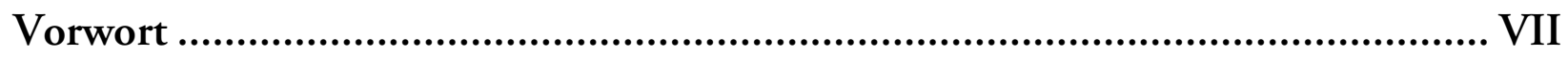

Abbildungsverzeichnis .................................................................................

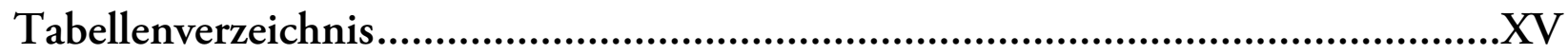

Abkürzungsverzeichnis.....................................................................................

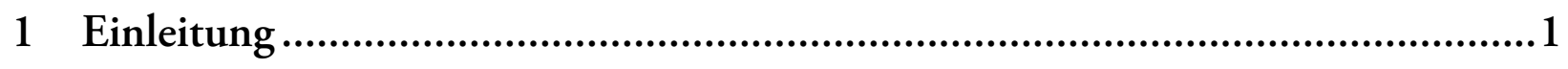

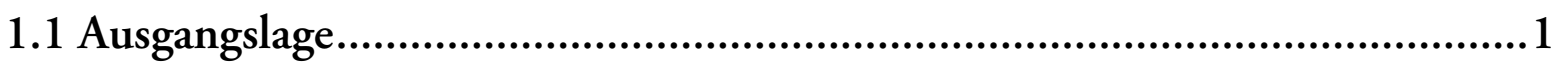

1.2 Problemstellung und Forschungsfrage ..................................................2

1.3 Theoretischer Ansatz der Arbeit................................................................4

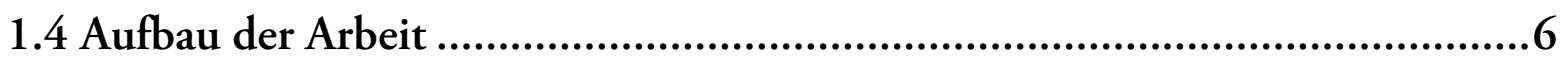

2 Theoretischer Bezugsrahmen zu strategischen Entscheidungen und pfadbedingter Rigidität ...........................................................................11

2.1 Organisation und Management: Begriffliche Grundlagen ..........................11

2.1.1 Zum Begriff der Organisation ..................................................... 11

2.1.2 Grundlagen des Managements ............................................. 13

2.1.2.1 Komplexität als modernes Managementproblem......................... 16

2.1.2.2 Dynamik als modernes Managementproblem ............................. 18

2.2 Strategische Entscheidungen: Theoretische Grundlagen..............................20

2.2.1 Zum Begriff der Entscheidung ................................................. 20

2.2.2 Zum Begriff der Strategie ..................................................... 22

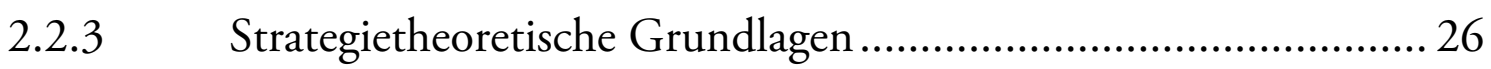

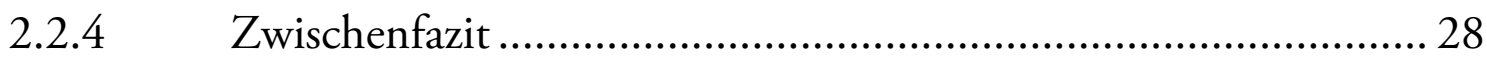

2.3 Theorie der Pfadabhängigkeit ...................................................................29

2.3.1 Ursprung und konzeptionelle Öffnung der Pfadtheorie ............. 29 
2.3.2 Fokussierung: Pfadabhängigkeit im engeren Sinne..................... 35

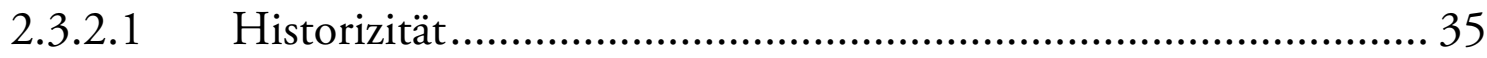

2.3.2.2 Der Mechanismus der positiven Rückkopplung........................ 36

2.3.2.2.1 Zum Begriff des Mechanismus................................................. 36

2.3.2.2.2 Selbstverstärkende Effekte und positive Rückkopplung.............. 39

2.3.2.3 Lock-In und Rigidität .............................................................. 46

2.3.2.4 Zwischenfazit zur Theorie der Pfadabhängigkeit........................ 50

2.4 Theoriediskussion zu organisationaler Trägheit.........................................51

$2.4 .1 \quad$ Imprinting ..................................................................... 52

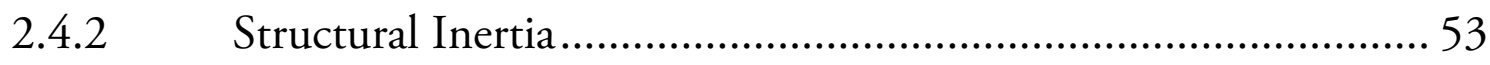

2.4.3 Lerntrajektorien und Kernrigiditäten ………............................... 55

2.4.4 Kognitive Verfestigungen ....................................................... 58

2.4.5 Konzeptionelle Zusammenführung............................................. 60

2.5 Konzeption strategischer Pfadabhängigkeit .................................................64

3 Der Fallstudienansatz als Forschungsstrategie.................................................69

3.1 Fallstudien und ihre Abgrenzung zu alternativen Forschungsstrategien ......69

3.2 Grundtypen eines Fallstudienaufbaus .........................................................73

3.2.1 Fall, Kontext und Analyseobjekt .............................................. 75

3.2.2 Einzel- und Mehrfallstudie....................................................... 76

3.3 Qualitätsansprüche an die Fallstudienforschung ..........................................77

3.4 Bertelsmann Buchclub: Eine holistische Einzelfallstudie............................79

3.5 Datenerhebung und -auswertung..............................................................82

3.5.1 Multiple Datenquellen und Triangulation ................................. 82

3.5.1.1 Dokumente und Archivdaten................................................... 84

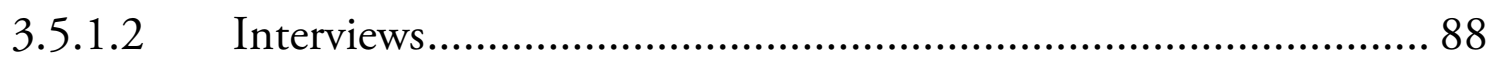

3.5.2 Fallstudiendatenbank und Nachvollziehbarkeit......................... 90

$4 \quad$ Fallstudie zum Bertelsmann Buchclub .....................................................95

4.1 Das Buch und das deutsche Buchhandelssystem ............................................95

4.1.1 Zum Begriff des Buches ............................................................ 96

4.1.2 Der Buchhandel als Mittler zwischen Autoren und Lesern......... 98 
4.1.3 Gesetz über die Preisbindung für Bücher ................................. 103

4.2 Buchgemeinschaften als Mitgliedschaftssysteme......................................105

4.2.1 Definition und Besonderheiten ................................................. 105

4.2.2 Historische Entwicklung der Buchgemeinschaften .................... 108

4.3 Der Bertelsmann Buchclub und die Bertelsmann AG: Historische Entwicklung und Meilensteine ............................................................111

4.3.1 Vom Lesering zur Direct Group................................................. 112

4.3.1.1 Der C. Bertelsmann-Verlag (1835-1950) ................................ 112

4.3.1.2 Gründung und Aufbau (1950-1970)....................................... 115

4.3.1.3 Übernahmen und Auslandsexpansion (1970-1990) .................. 119

4.3.1.4 Öffnung und Refokussierung (1990-2007) .............................. 121

4.3.2 Der Bertelsmann Konzern als Systemumwelt ............................ 124

4.3.2.1 Arvato: Druck, Dienstleistung, Onlinebetriebe ......................... 125

4.3.2.2 Random House: Buchverlage ................................................... 127

4.3.2.3 Gruner+Jahr: Zeitschriften und Zeitungen.............................. 131

4.3.2.4 Sony BMG: Musikvermarktung ............................................ 132

4.3.2.5 RTL-Group: Film, Fernsehen, Hörfunk .................................. 134

4.3.3 Zwischenfazit zur Bertelsmann AG ....................................... 138

4.4 Pfadtheoretische Analyse der strategischen Entwicklung ...........................140

4.4.1 Operationalisierung der Pfadelemente.................................... 140

4.4.2 Schritt 1: Rigiditätsnachweis .................................................. 147

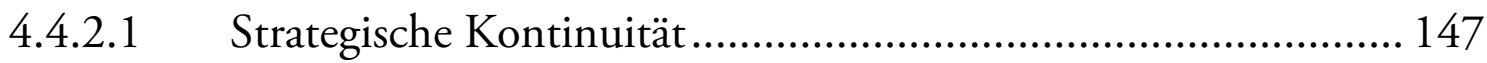

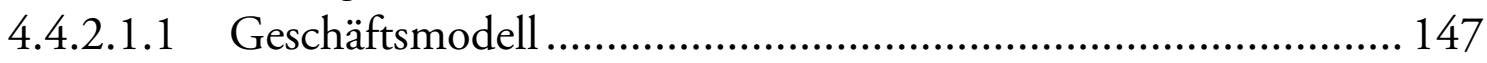

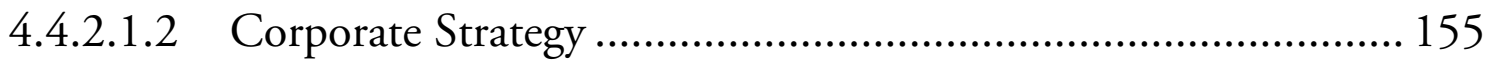

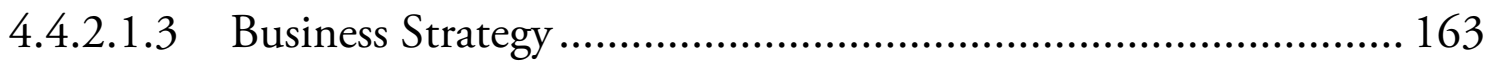

4.4.2.1.4 Zwischenfazit zu strategischer Kontinuität ............................... 167

4.4.2.2 Umweltdynamik .................................................................... 169

4.4.2.2.1 Wettbewerb unter den Anbietern ......................................... 170

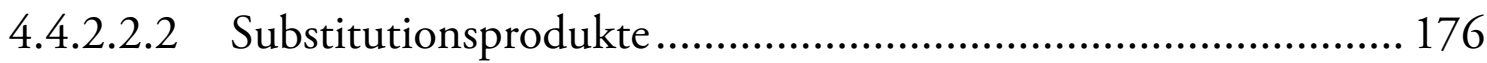

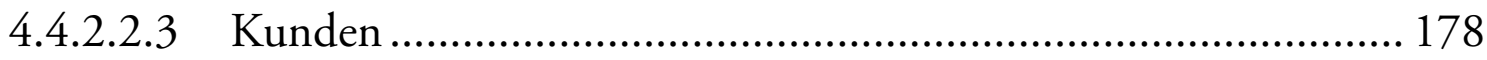

4.4.2.2.4 Politische Rahmenbedingungen ............................................. 179

4.4.2.2.5 Zwischenfazit zu Umweltdynamik ............................................ 181

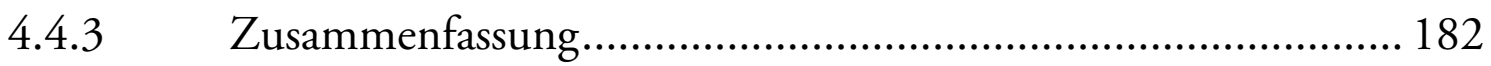




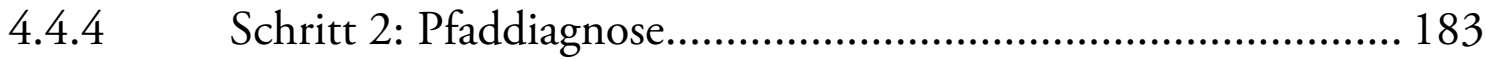

4.4.4.1 Komponente 1: Historizität ..................................................... 183

4.4.4.2 Komponente 2: Critical Juncture ............................................ 186

4.4.4.3 Komponente 3: Positive Rückkopplung................................. 187

4.4.4.3.1 Economies of Scale und Risikominimierung ............................ 187

4.4.4.3.2 Zwischenfazit zu Economies of Scale und Risikominimierung. 207

4.4.4.3.3 Komplementaritätseffekte ..................................................... 209

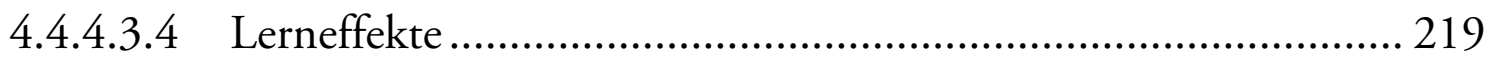

4.4.4.3.5 Zwischenfazit zu Komplementaritäts- und Lerneffekten .......... 222

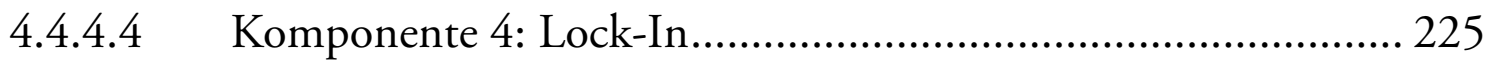

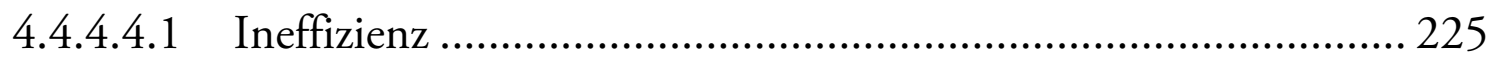

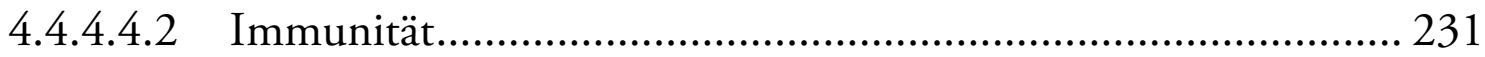

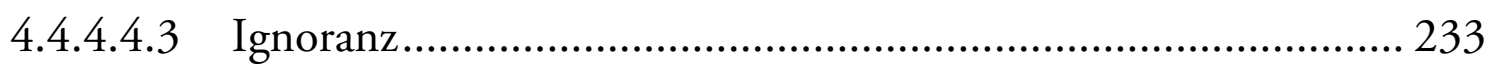

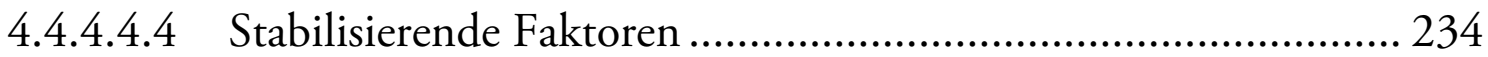

4.4.4.4.5 Zwischenfazit zum Lock-In..................................................... 240

4.4.5 Zusammenfassung und Einordnung in das Drei-PhasenModell der Pfadentstehung ................................................... 241

4.4.5.1 Phase I: Historizität........................................................... 242

4.4.5.2 Phase II: Critical Juncture und Effekte der Selbstverstärkung... 243

4.4.5.3 Phase III: Lock-In, verstärkende und stabilisierende Effekte..... 245

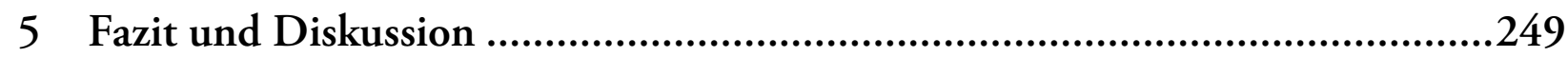

5.1 Zusammenfassung der Ergebnisse.............................................................249

5.2 Zuverlässigkeit der Ergebnisse ..................................................................252

5.3 Weiterführende Diskussion ..................................................................254

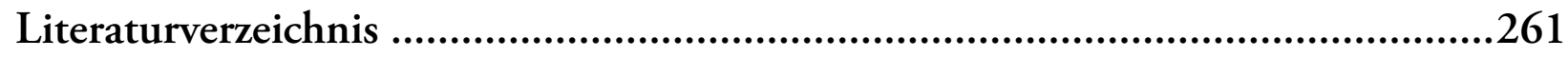

Anhang 1: Stichwortkataloge ..............................................................................28

Anhang 2: Beispiel eines Interviewleitfadens ...................................................287

Anhang 3: Chronologie des Bertelsmann Buchclubs Deutschland........................289 


\section{Abbildungsverzeichnis}

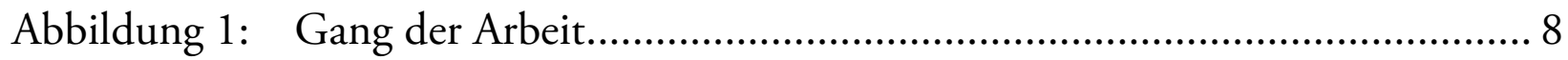

Abbildung 2: Moderner Managementprozess........................................................ 19

Abbildung 3: Idealtypischer Entscheidungsprozess ............................................... 21

Abbildung 4: Drei Ebenen des Strategiebegriffs ................................................... 24

Abbildung 5: Strategie als Verbindung zwischen Unternehmen und Umwelt........ 25

Abbildung 6: Wirkrichtungen kausaler Prozessketten ............................................. 38

Abbildung 7: Drei-Phasen-Modell der Pfadentstehung.......................................... 49

Abbildung 8: Grundtypen eines Fallstudienaufbaus............................................... 74

Abbildung 9: Einfaches inhaltsanalytisches Kommunikationsmodell ...................... 83

Abbildung 10: Buchhandel zwischen Autor und Leser .............................................. 99

Abbildung 11: Fokales System ,Buchclub Deutschland' und Kontextfaktoren der Bertelsmann AG ....................................................................... 139

Abbildung 12: Übersicht der Corporate Strategy anhand der Produkt/MarktMatrix

Abbildung 13: Hauptwettbewerber des Bertelsmann Buchclubs im Jahr 2007 ...... 176

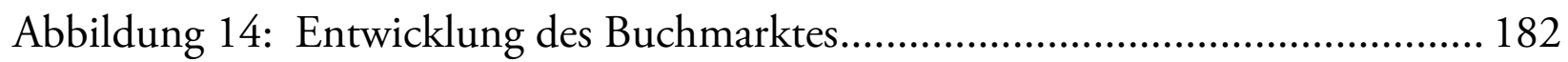

Abbildung 15: Strategischer Fit zwischen Umwelt und System ............................. 190

Abbildung 16: Absatzentwicklung belletristischer Bücher ...................................... 194

Abbildung 17: Stückkosten-Degressionseffekte der Buchherstellung...................... 199

Abbildung 18: Gewinn in Abhängigkeit der Kalkulationsform und Auflage .......... 206

Abbildung 19: Erfolgsspirale des Buchclubs .......................................................... 208

Abbildung 20: Trägheitsspirale des Buchclubs ..................................................... 224

Abbildung 21: Entwicklung des Mitgliedsbestands des Bertelsmann Buchclubs Deutschland

Abbildung 22: Drei-Phasen-Modell der Pfadentstehung am Beispiel des Buchclubs 



\section{Tabellenverzeichnis}

Tabelle 1: Klassifikationsschema für Unternehmensstrategien .........................28

Tabelle 2: Formen von Selbstverstärkungsmechanismen.................................. 41

Tabelle 3: $\quad$ Vergleichende Einordnung der Theorien zu

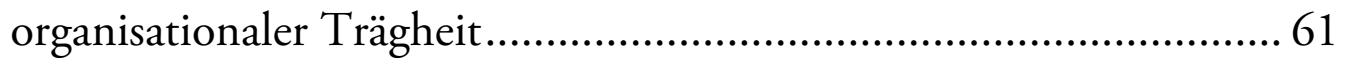

Tabelle 4: Rigiditätsnachweis als Schritt 1 der Pfadanalyse ................................ 65

Tabelle 5: $\quad$ Pfaddiagnose als Schritt 2 der Pfadanalyse........................................6 68

Tabelle 6: Abgrenzung der Fallstudie von alternativen Forschungsansätzen .......71

Tabelle 7: Datenbasis aus Dokumenten und Archivmaterial............................. 86

Tabelle 8: Datenbasis aus problemzentrierten Experteninterviews .................... 90

Tabelle 9: $\quad$ Auszug aus der Fallstudiendatenbank ……......................................... 93

Tabelle 10: Operationalisierung strategischer Rigidität als Schritt 1 der Pfadanalyse .............................................................................. 142

Tabelle 11: Operationalisierung der Elemente zur Pfaddiagnose als Schritt 2 der Pfadanalyse ........................................................................... 146

Tabelle 12: Beispielrechnung der Stückkostendegression der Buchherstellung.. 198

Tabelle 13: Hochrechung der Verkaufsauflage des Hauptvorschlagsbands

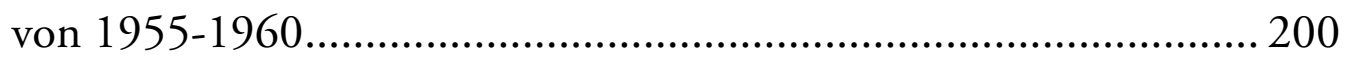

Tabelle 14: Kalkulationsschemata von Verlag, einstufiger und zweistufiger Buchgemeinschaft 



\section{Abkürzungsverzeichnis}

Aufl.

BG

BI

BUA

et al.

FAS

FAZ

FR

FTD

Hrsg.

LRR

o. ä.

o. D.

o. V.

SZ

Verf.

WIC

$\mathrm{W} \& \mathrm{~V}$
Auflage

Bertelsmann Geschäftsbericht

Bertelsmann Illustrierte

Bertelsmann Unternehmensarchiv

et alii

Frankfurter Allgemeine Sonntagszeitung

Frankfurter Allgemeine Zeitung

Frankfurter Rundschau

Financial Times Deutschland

Herausgeber

Lesering-Rundbrief

oder ähnliches

ohne Datum

ohne Verfasser

Süddeutsche Zeitung

Verfasser

Wir im Club (Magazin für Bertelsmann Clubmitarbeiter)

Werben \& Verkaufen 



\section{$1 \quad$ Einleitung}

Das einleitende Kapitel stellt zunächst die empirische Ausgangslage dar, auf deren Basis der theoretische Forschungsbedarf aufgebaut und die Forschungsfrage abgeleitet werden. Es folgen eine Übersicht über den der Arbeit zugrunde liegenden theoretischen Forschungsansatz und ein zusammenfassender Ausblick auf das Forschungsvorhaben. Die Einleitung schließt mit einem chronologischen Ausblick auf den Aufbau der Arbeit.

\subsection{Ausgangslage}

Der Bertelsmann Buchclub kann zweifelsohne als die Keimzelle der heutigen Bertelsmann AG, Deutschlands größtem, international agierenden Medienkonzern, bezeichnet werden. Gegründet im Jahr 1950 beschrieb die ertragsreiche Buchgemeinschaft eine beispielslose Erfolgsgeschichte. Die Bücher des Buchclubs füllten innerhalb nur weniger Jahre die Regale in Millionen von deutschen Haushalten und befriedigten gleichermaßen den Drang nach Unterhaltung und Bildung in der Bevölkerung.

Der Glanz der Gründungs- und Aufbaujahre des Buchclubs wurde jedoch sukzessive von sinkenden Umsätzen, Imageverlust und Mitgliederschwund abgelöst. Den wirtschaftlichen Schwierigkeiten der Buchgemeinschaft von Bertelsmann entzog sich auch die mediale Öffentlichkeit nicht. Seit Mitte der 80er wurden immer wieder kritische Stimmen laut, welche die Ertragsschwäche und strukturelle Krise des Buchclubs benannten:

"Clubgeschäft verspricht keine weitere Expansion“ (1988), ${ }^{1}$

„Das Buchclub-Geschäft scheint ausgereizt.“ (1995), ${ }^{2}$

„Buchclub bremst Bertelsmann“ (1998), ${ }^{3}$

"Kunden verzweifelt gesucht" (2005), ${ }^{4}$

„Bertelsmann ringt um Buchclub-Profite“ (2006). ${ }^{5}$

1 Handelsblatt (1988, 29.02.), S. 25.

2 Handelsblatt $(1995,23.06)$, S. 12.

3 Die Welt $(1998,20.11)$.

$4 \quad$ W\&V $(2005,28.04)$, S. 10. 
Anfänglich verhalten, zuletzt auch expressis verbis, sieht sich die traditionsreiche Unternehmung Buchclub der Diskussion ausgesetzt, „fast schon ein Auslaufmodell“ ${ }^{\text {“6 }} \mathrm{zu}$ sein.

Angesichts dieser öffentlichen Diskussionen, langjähriger Verluste und Mitgliedererosionen überrascht die Betrachtung der strategischen Entwicklung des Bertelsmann Buchclubs: Die grundsätzliche Geschäftsstrategie ist seit mehr als 50 Jahren kaum verändert worden und zeichnet sich durch ein hohes Maß an Gleichförmigkeit und Kontinuität aus. Zwar sprechen Managementwechsel und Reorganisationsprozesse für Bemühungen, die Ertragswende strategisch einleiten zu wollen, allerdings ohne eine grundsätzliche Neuausrichtung des Clubgeschäfts nachhaltig forciert zu haben.

Das Festhalten an der tradierten strategischen Ausrichtung des Buchclubs trotz massiver wirtschaftlicher Schwierigkeiten ist aus rational ökonomischer Perspektive kaum zu erklären. Vor diesem Hintergrund werden fernab rationaler Entscheidungskalküle häufig emotionale Ursachen für das persistente strategische Entscheidungsverhalten des Buchclubs angeführt. Besonderes Gewicht liegt dabei auf der Tatsache, dass die Wurzeln des Bertelsmann Konzerns zur Entstehungsgeschichte des Buchclubs zurückführen. So interessant wie der Gründungseinfluss aus unternehmenshistorischer Perspektive auf die strategische Entwicklung ist, um so interessanter ist die Erforschung der augenscheinlich rational nicht zu erklärenden strategischen Persistenz aus einer Perspektive des strategischen Managements. Dieser Beitrag soll in der vorliegenden Arbeit geleistet werden.

\subsection{Problemstellung und Forschungsfrage}

Management bedeutet Steuerung. Steuerung wiederum setzt Handlungsautonomie voraus. Je nach Situation müssen alte Wege fortgesetzt, allerdings auch - sofern es die

FTD (2006, 06.06.), S. 6.

Handelsblatt (2005, 22.03.), S. 21. 
Umstände erfordern - Richtungswechsel erfolgen und neue Wege beschritten werden können. Um ein Unternehmen zu steuern, strategische Entscheidungen zur Sicherung langfristiger Wettbewerbsvorteile zu treffen, ist es daher unabdingbar, über Flexibilität und Entscheidungsfreiheit zu verfügen. Das Ziel von Management muss daher sein, ein Mindestmaß an Handlungs- oder Entscheidungsfreiheit zu sichern, um sich wandelnden Markt- und Wettbewerbsbedingungen anpassen oder aktiv als Treiber neuer Marktentwicklungen agieren zu können.

Dass dies nicht immer selbstverständlich ist, zeigen zahlreiche Beispiele von Unternehmen, welche in der Vergangenheit sehr erfolgreich geführt wurden, jedoch ihre Flexibilität einbüßten und zunehmend handlungsunfähig wurden.7 Perioden lang anhaltenden Erfolgs oder der langfristige Ausbau spezifischer Kernkompetenzen bergen z. B. die Gefahr, resistent gegenüber notwendigen Veränderungsprozessen zu werden und in eine Trägheitsfalle zu geraten. Wichtig ist dabei, dass kontinuierliche Entscheidungsverläufe nicht per se mit Rigidität und Handlungsunfähigkeit einhergehen müssen: Unter stabilen Umweltbedingungen und Wettbewerbsvorteilen kann die Kontinuität von Unternehmensentscheidungen durchaus vorteilhaft sein. Unternehmen können spezifische Kernkompetenzen entwickeln, spezielle, unimitierbare Technologien hervorbringen und sich über lange Zeit mit derselben Strategie erfolgreich im Markt positionieren und behaupten. Problematisch wird die strategische, Trajektorie' erst, sobald sich die Umweltbedingungen ändern, der Markt dynamisch ist und strategischer Handlungsbedarf, z. B. durch lang anhaltende wirtschaftliche Verluste, entsteht. Sofern also strategische Kontinuität und Handlungsmonotonie eines Unternehmens auf die Notwendigkeit zum Wandel trifft, ist von Trägheit und Rigidität auszugehen, wodurch das langfristige Überleben der Unternehmung gefährdet sein kann.

7 Vgl. hierzu exemplarisch Tripsas, M./Gavetti, G. (2000), S. 1147-1161. 
Diese Phänomene von strategischer Rigidität und Trägheit - wie im Fall des Bertelsmann Buchclubs - stehen im Zentrum der vorliegenden Arbeit.

Neben der ökonomischen Brisanz - nicht selten mündet strategische Rigidität in der Existenzbedrohung von Unternehmen - ist die Entscheidungspersistenz wirtschaftlich ineffizienter Strategien aus einem ökonomischen Rationalitätskalkül nicht zu erklären und bietet daher eine viel versprechende Oberfläche zur Generierung neuer Forschungserkenntnisse.

Ziel dieser Arbeit ist es daher, exemplarisch am Beispiel des Bertelsmann Buchclubs aufzuzeigen, warum eine Organisation langfristig an verlustreichen Strategien festhält. Ein Schwerpunkt der Untersuchung liegt dabei auf der Analyse der zur Trägheit führenden Entscheidungsprozesse. Das Einnehmen einer Prozessperspektive zur Betrachtung von Rigidität mag paradox erscheinen, legt ,Rigidität' doch gerade die Konnotation mit Statik, also Nicht-Bewegung oder Nicht-Wandel, nahe. Rigidität als Form von Nicht-Wandel zeichnet sich zwar durch eine inhaltliche Gleichförmigkeit aus, die allerdings nur durch die Erhebung zu mindestens zwei unterschiedlichen Zeitpunkten zu messen ist. Für eine fundierte Analyse von Rigiditätsphänomenen ist es somit zwingend notwendig, eine Prozessperspektive einzunehmen.

Zur Beantwortung der zentralen Forschungsfrage „Warum hält eine Organisation langfristig an verlustreichen Strategien fest?" greift die vorliegende Arbeit daher auf einen theoretischen Ansatz zurück, der Rigiditätsphänomene auf der Grundlage ihres eigenen Entstehungsprozesses selbst (prozessendogen) zu erklären vermag - die Theorie der Pfadabhängigkeit.

\subsection{Theoretischer Ansatz der Arbeit}

Ihren Ursprung in der ökonomischen Wissenschaftsdisziplin nahm die Pfadtheorie Mitte der 80er Jahre durch die zentralen Artikel von DAVID (1985) und ARTHUR (1989), welche die pfadbedingte Durchsetzung von technologischen Standards unter 
Ausschluss konkurrierender Technologien modelltheoretisch (ARTHUR) sowie empirisch (DAVID) erklärten. ${ }^{8}$ Zusammen mit der Übertragung der Pfadtheorie in institutionelle ${ }^{9}$ und organisationale, ${ }^{10}$ politische ${ }^{11}$ und gesellschaftliche ${ }^{12}$ Wissensbereiche erfolgte sukzessive eine konzeptionelle Ausweitung der pfadtheoretischen Annahmen.

In Anlehnung an SCHREYÖGG ET AL. (2003) wird dieser inflationären Erweiterung mit der Einführung eines engen Pfadverständnisses begegnet. ${ }^{13}$ Dieses basiert auf vier zentralen Komponenten: Zum einen sind pfadabhängige Prozesse zwar historisch vorgeprägt, jedoch in der Anfangsphase nahezu kontingent, da eine Vielzahl von Handlungsmöglichkeiten besteht. Die Ergebnisse von Entscheidungsprozessen sind in dieser frühen Phase weder vorhersagbar noch determiniert. Durch das Auftreten eines kritischen Ereignisses (Critical Juncture) als zweite Komponente wird der Mechanismus positiver Rückkopplungen als dritte zentrale Pfadkomponente ausgelöst, dessen selbstverstärkende Kräfte die (strategischen) Handlungsmöglichkeiten des Unternehmens fortlaufend reduzieren. Abweichendes Entscheidungsverhalten ist noch möglich, allerdings immer unwahrscheinlicher. Schließlich wird der Zustand eines Lock-Ins als vierte Pfadkomponente erreicht, welcher sich in kaum zu ändernden, organisationalen Beharrungstendenzen, Rigidität und Wandlungswiderständen äußert - auch, wenn sich eine ökonomisch inferiore Entscheidungsalternative durchgesetzt hat. ${ }^{14}$ Phänomene organisationaler Trägheit und Rigidität werden somit von der Theorie der Pfadabhängigkeit aus dem Entstehungsprozess selbst heraus erklärt. Das beobachtbare Ergebnis einer pfadabhängigen Entwicklung, der Lock-In, wird unter Berücksichtigung der dazu führenden Prozessdynamik betrachtet.

8 Vgl. David, P.A. (1985), Arthur, W.B. (1989).

9 Vgl. North, D. (1990).

10 Vgl. Schreyögg, G. (2003).

11 Vgl. Pierson, P. (2000).

12 Vgl. Mahoney, J. (2000).

13 Vgl. Schreyögg, G. et al. (2003).

14 Vgl. Sydow, J. et al. (2005), S. 5 f. 
In der vorliegenden Arbeit wird die strategische Entwicklung des Bertelsmann Buchclubs über den Zeitraum von 1950 bis 2007 aus einer pfadtheoretischen Perspektive analysiert. Schritt 1 überprüft die Vermutung einer strategischen Rigidität, worauf aufbauend Schritt 2 untersucht, ob sich der strategische Entscheidungsverlauf durch pfadabhängige Prozesse erklären lässt.

\subsection{Aufbau der Arbeit}

Nach der Einleitung (Kapitel 1) werden im 2. Kapitel die theoretischen Grundlagen zur Analyse des Forschungsgegenstandes vorgestellt: Zunächst erfolgt eine definitorische Einordnung zu den Begriffen Organisation, Management (Kapitel 2.1) und strategischen Entscheidungen (Kapitel 2.2). Kapitel 2.3 schließt mit einer ausführlichen Theoriediskussion zur Pfadabhängigkeit an, welche im darauf folgenden Kapitel 2.4 mit den zentralen theoretischen Ansätzen zu organisationaler Trägheit kontrastiert und konzeptionell zusammengeführt wird. Gemeinsamkeiten und Unterschiede werden herausgearbeitet. Der Theorieteil endet mit einem konzeptionellen Modell zur Analyse strategischer Pfadabhängigkeit (Kapitel 2.5).

Im Anschluss an die Theoriediskussion wird in Kapitel 3 das methodische Vorgehen ausführlich dargelegt. Nach einer kurzen Erörterung der Forschungsfrage wird die Forschungsstrategie der Fallstudie erläutert und mit alternativen Forschungsdesigns kontrastiert (Kapitel 3.1). Hierauf aufbauend werden unter Kapitel 3.2 die Grundtypen des Fallstudienaufbaus eingeführt und die zentralen Qualitätskriterien zu dessen Beurteilung vorgestellt (Kapitel 3.3). Nachdem die einzelnen Merkmale der Fallstudienstrategie auf einer theoretischen Ebene erarbeitet worden sind, werden diese im folgenden Kapitel 3.4 auf den empirischen Fall, den Bertelsmann Buchclub, übertragen. Der methodische Teil schließt in Kapitel 3.5 mit einer detaillierten Beschreibung der Datenerhebungsprinzipien sowie der Datenquellen ,Dokumente', ,Archivdaten’ und ,Interviews'. 
Im empirischen Teil unter Kapitel 4 werden die konkreten Ergebnisse der pfadtheoretischen Analyse im Fall des Bertelsmann Buchclubs dargelegt. Einleitend werden zunächst grundlegende Fakten zum Produkt Buch, dem deutschen Buchhandelssystem (Kapitel 4.1) sowie Buchgemeinschaften (Kapitel 4.2) erörtert. Es folgt eine ausführliche Beschreibung der historischen Entwicklung des Bertelsmann Buchclubs sowie der weiteren Divisionen der Bertelsmann AG (Kapitel 4.3). Auf Basis der historischen Daten erfolgt in Anlehnung an das im Theorieteil entwickelte Analysemodell unter Kapitel 4.4 die zentrale Pfadanalyse des Bertelsmann Buchclubs in zwei Schritten. Als Ausgangspunkt der Pfadanalyse werden die zu erhebenden Indikatoren vorgestellt (Kapitel 4.4.1). Zum Nachweis von strategischer Rigidität (Kapitel 4.4.2) werden in Schritt 1 der Pfadanalyse die Ergebnisse zu strategischer Kontinuität (Kapitel 4.4.1.1) und Umweltdynamik (Kapitel 4.4.1.2) diskutiert. Als Schritt 2 schließt sich in Kapitel 4.4.4 eine detaillierte Darstellung der Pfaddiagnose anhand der vier Komponenten Historizität (Kapitel 4.4.4.1), Critical Juncture (Kapitel 4.4.4.2), positive Rückkopplung (Kapitel 4.4.4.3) sowie Lock-In (Kapitel 4.4.4.4) an. Im darauf folgenden Kapitel 4.4.5 werden die Ergebnisse zusammengeführt und in das Drei-PhasenModell der Pfadentstehung eingeordnet, bevor die Arbeit in Kapitel 5 mit einem zusammenfassenden Fazit und einer weiterführenden Diskussion abschließt. 
Abbildung 1: Gang der Arbeit

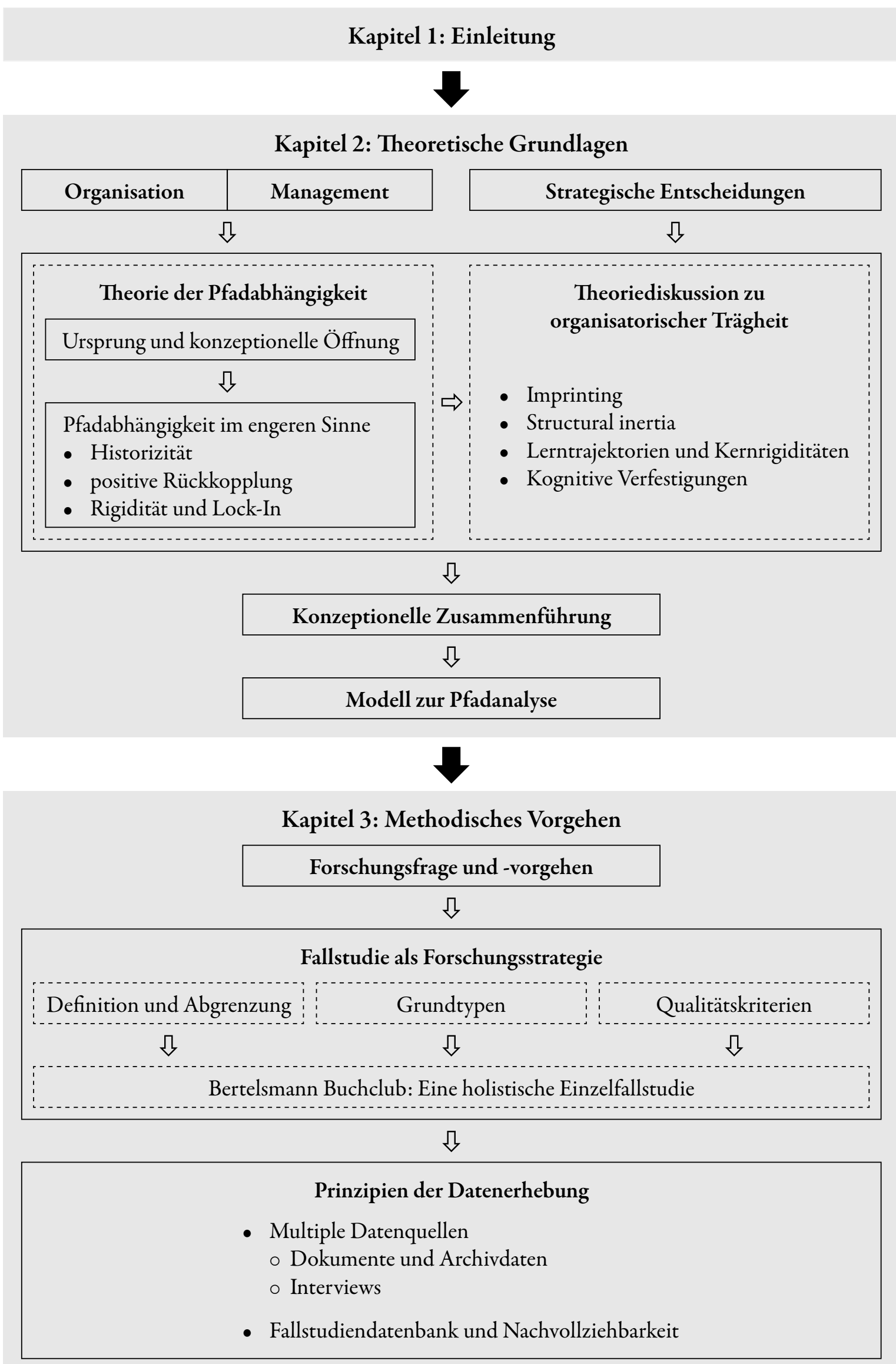




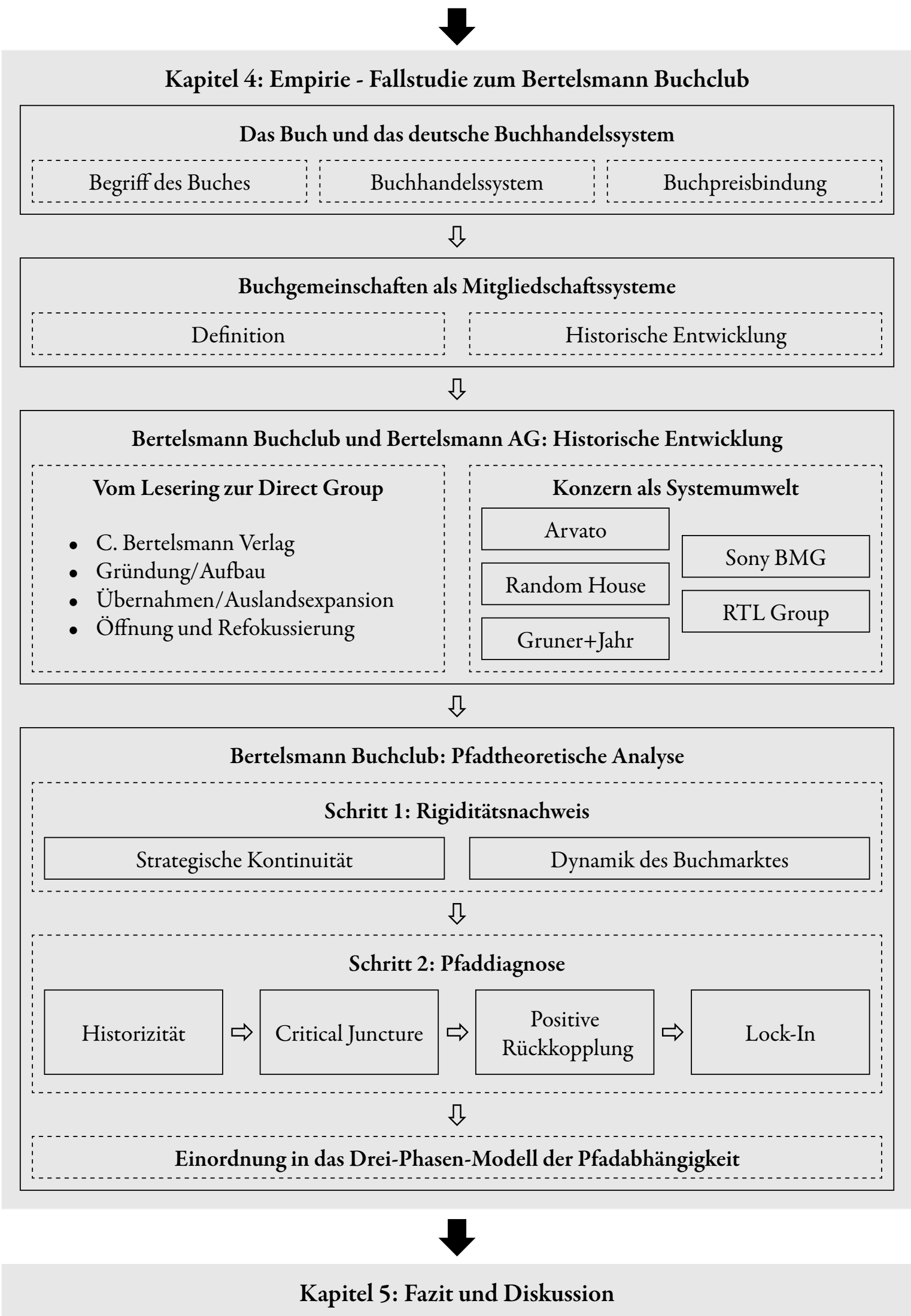

Quelle: Eigene Darstellung. 



\section{Theoretischer Bezugsrahmen zu strategischen Entscheidungen und pfadbedingter Rigidität}

Im Rahmen dieses Kapitels sollen die theoretischen Grundlagen, auf denen die empirische Pfadanalyse in Kapitel 4 basiert, vorgestellt werden. Nach Klärung der zentralen Begriffe und definitorischen Einordnungen erfolgt eine umfangreiche Theoriediskussion zu Pfadabhängigkeit in Abgrenzung zu alternativen Erklärungsansätzen von organisationaler Trägheit. Die Theoriediskussion mündet schließlich in der Entwicklung eines konzeptionellen Analysemodells von strategischer Pfadabhängigkeit, das in der empirischen Pfadanalyse in Kapitel 4 zur Anwendung kommt.

\subsection{Organisation und Management: Begriffliche Grundlagen}

Zur Beantwortung der Forschungsfrage, warum eine Organisation langfristig an verlustreichen Strategien festhält, soll zunächst das definitorische Grundverständnis der zentralen Begriffe ,Organisation' und ,Management' vorgestellt werden. Neben der Festlegung einer klaren Arbeitsdefinition wird so bereits die Perspektive, aus welcher die pfadtheoretische Analyse erfolgen soll, deutlich.

\subsubsection{Zum Begriff der Organisation}

Der Organisationsbegriff hat neben seiner wissenschaftlichen Verwendung längst Einzug in die Alltagssprache gehalten, so dass die Notwendigkeit einer engen definitorischen Abgrenzung für die vorliegende Arbeit immanent ist.

Vor allem zwei Ansätze zur Beschreibung von Organisationen haben sich durchgesetzt: Der instrumentelle und der institutionelle Begriff. Während nach instrumentellem Verständnis eine Organisation als strukturelle Regelung und Führungsinstrument aufzufassen ist, weitet der institutionelle Organisationsbegriff die Perspektive auf die 
ganze Institution bzw. das ganze System aus. ${ }^{15}$ Letzterem Begriffsverständnis folgt auch die vorliegende Arbeit, da durch die Abbildung des gesamten Systems nicht nur die formale, planmäßig entworfene Organisationsstruktur eingefangen, sondern der Blickwinkel auf die für die Pfadanalyse wichtigen ungeplanten, emergenten und dysfunktionalen Prozesse ausgeweitet werden kann. ${ }^{16}$

In diesem Sinne basiert das institutionelle Organisationsverständnis auf drei zentralen Merkmalen:

Zunächst unterliegen Organisationen einer spezifischen Zweckorientierung. ${ }^{17} \mathrm{Im}$ Falle des Bertelsmann Buchclubs handelt es sich um eine erwerbswirtschaftlich ausgerichtete Organisation, eine Unternehmung. Im Gegensatz zu Non-ProfitOrganisationen unterliegen Unternehmungen produktiv-wirtschaftlichen Zwecken als übergeordneter Zielsetzung. ${ }^{18}$ Auch diese Zielausrichtung ist nicht monolithisch zu sehen, sondern kann als Zielsystem verstanden werden, dessen einzelne Unterziele sich unter Umständen partiell widersprechen (Liquidität vs. Rentabilität). ${ }^{19}$

Zur Zielerreichung bilden geregelte Arbeitsteilung und -koordination - manifestiert in Organisationsstrukturen - das zweite Merkmal von Organisationen. ${ }^{20}$ Die Handlungen von Personen als Elemente von Organisationen werden so nach bestimmten Mustern differenziert und mit Rollenerwartungen zur Zielerfüllung belegt.

Schließlich definieren sich Organisationen durch eine Grenzziehung zur Umwelt als drittes Merkmal..21 Organisationen haben keine natürlichen Grenzen, sondern schaffen diese durch Differenzbildung autonom und absichtsvoll. Die Unterscheidung zwischen organisatorischer Innenwelt und Umwelt impliziert auch die Zuordnung

\footnotetext{
15 Vgl. Schreyögg, G. (2006), S. 4-11.

16 Vgl. Schreyögg, G. (2006), S. 11.

17 Vgl. Schreyögg, G. (2006), S. 9f.

18 Vgl. Ulrich, H. (1970), S. 166.

19 Vgl. Schreyögg, G. (1991), S. $268 f$.

20 Vgl. Schreyögg, G. (2006), S. 10.

${ }^{21}$ Vgl. Schreyögg, G. (2006), S. 11.
} 
von Organisationsmitgliedern und Nicht-Mitgliedern als Teile der Außen- oder Umwelt.

Das Kriterium der Grenzziehung zur Umwelt deutet bereits auf den Systemcharakter von Organisationen hin. Systeme bestehen aus Elementen und deren Beziehungen zueinander. Im Hinblick auf die Beziehungen zwischen den Elementen ist also ein System stets mehr als die Summe seiner einzelnen Elemente. Unternehmen als zweckbezogene Organisationen lassen sich speziell als soziale bzw. Handlungssysteme beschreiben:22 Die einzelnen Systemelemente bilden miteinander verbundene menschliche Handlungen, so dass Probleme durch kollektive Leistungsprozesse gelöst werden. ${ }^{23}$

Nach diesen einführenden Aussagen zum Systemcharakter von Organisationen und Unternehmungen soll im nächsten Kapitel auf den Begriff des Managements und - damit verbunden - den Managementprozess eingegangen werden. Wie einleitend erwähnt, soll der Bertelsmann Buchclub aus der Perspektive des strategischen Managements analysiert werden, so dass zunächst das managementtheoretische Grundverständnis geklärt werden muss, auf dem die Theorie der Pfadabhängigkeit in der vorliegenden Arbeit fußt.

\subsubsection{Grundlagen des Managements}

Parallel zum Organisationsbegriff haben sich in der Managementlehre ebenfalls zwei maßgebliche Ansätze durchgesetzt:

Management als Institution und Management als Funktion. ${ }^{24}$ Während ein institutionelles Managementverständnis auf einen spezifischen Personenkreis mit Führungs- und Anweisungsbefugnissen abstellt, bezieht sich der funktionale Ansatz, welcher der vorliegenden Arbeit zugrunde liegt, auf die Steuerung des Leistungsprozesses

22 Vgl. Steinmann, H./Schreyögg, G. (2005), S. 140.

23 Vgl. Schreyögg, G. (1984), S. 248.

24 Vgl. Steinmann, H./Schreyögg, G. (2005), S. 5-7; Staehle, W. (1999), S. 71-73; Ulrich, P./ Fluri, E. (1995), S. 13-15. 
innerhalb der Organisation. Management definiert sich in diesem Sinne als „Komplex von Steuerungsaufgaben, die bei der Leistungserstellung und -sicherung in arbeitsteiligen Systemen erbracht werden müssen. ${ }^{25}$ Management erhebt somit vor allem einen generellen Gestaltungsanspruch, eine Organisation an ihrer Zielsetzung ausrichten zu können. Steuerung und Gestaltung setzen demzufolge implizit einen Handlungs- oder Entscheidungsspielraum voraus, aus dem durch das Treffen bewusster (strategischer) Entscheidungen auf die Organisationsentwicklung Einfluss genommen werden kann. Obwohl diese Steuerungsaufgaben breit gefächert sind, lässt sich nach KoONTZ/O’DONNELL (1995) ein grober Kanon an fünf Managementfunktionen subsumieren: Planung, Organisation, Personaleinsatz, Führung und Kontrolle. ${ }^{26}$

Gemäß der traditionellen Managementlehre bauen die einzelnen Funktionen logisch aufeinander auf und müssen als Ablaufmodell verstanden werden. Hierbei nimmt die Funktion der Planung den logischen Anfang und erhält im Vergleich zu den weiteren Managementfunktionen einen Sonderstatus. ${ }^{27}$ Im Rahmen der Planung werden die zu erreichenden Ziele als Soll-Größen festgelegt und daraus diejenigen Wege abgeleitet, welche die Ziele optimal erfüllen können. Neben der Festlegung von langfristigen Zielen umfasst die Planung auch kurzfristige Programme und Richtlinien zur Programmumsetzung für die Gesamtunternehmung und einzelne Teilbereiche. ${ }^{28}$

25 Steinmann, H./Schreyögg, G. (2005); S. 7.

26 Vgl. Koontz, H./O’Donnell, C. (1955).

27 In diesem Zusammenhang wird in der klassischen Managementliteratur vom ,Primat der Planung' gesprochen. Vgl. hierzu Koontz, H./O’Donnell, C. (1955); Steinmann, H./Schreyögg, G. (2005), S. 131-139.

28 Nach der konzeptionell geprägten Phase der Planung erfolgt deren Realisation durch die zweite Managementfunktion, der Organisation. Ziel des Organisierens ist es, ein arbeitsteiliges Handlungsgefüge durch Stellen zu erschaffen, um Aufgaben und deren Verbindungen untereinander zu spezifizieren. Diese Stellen müssen im darauf folgenden Schritt mit Personal besetzt werden, dessen Erfüllung der dritten Managementfunktion, dem Personaleinsatz, zu Teil wird. Die nächste Managementaufgabe befasst sich mit der kontinuierlichen Anweisung und Veranlassung zur konkreten Arbeitsumsetzung durch die Wahrnehmung von Führungsaufgaben der Vorgesetzten. Der Soll/Ist-Vergleich im Rahmen der logisch letzten Managementfunktion, der Kon- 
Die schrittweise Abfolge der einzelnen Funktionen im traditionellen Managementprozess, und allen voran die Plandeterminiertheit zu Beginn des Prozesses, beruhen auf zentralen Prämissen, die mit der Konzeption der Pfadtheorie nur schwer in Einklang zu bringen sind. SCHREYÖGG (1991) verweist vor allem auf die modellhaften Prämissen der ,strukturierten Umwelt' und der ,Einzelaktor-Rationalität', die angesichts jüngerer theoretischer Erkenntnisse und empirischer Beobachtungen kaum durchzuhalten sind. ${ }^{29}$ Probleme wie Informationsmangel und Unsicherheit, Zielkonflikte und Implementationswiderstände werden somit per definitionem ausgeblendet und können nicht konzeptionell erschlossen werden.

Die Erweiterung des traditionellen Managementprozesses um zwei grundlegende Dimensionen - Komplexität und Dynamik - vermag große Teile der bestehenden Erklärungslücke zu füllen. Deren Einbezug und somit die Reformulierung des Managementprozesses aus systemtheoretischer Sicht erscheint in doppelter Hinsicht lohnenswert: Zum einen werden die Hauptkritikpunkte am traditionellen Managementkonzept aufgegriffen und verarbeitet, zum anderen in ein überarbeitetes Modell integriert und für die vorliegende empirische Fragestellung fruchtbar gemacht. Aufgrund der konzeptionellen Relevanz für die in Kapitel 4 behandelte Fallstudie wird im Folgenden auf die Integration von Komplexität und Dynamik in den Managementprozess näher eingegangen.

trolle, stellt sicher, ob die Pläne auch realisiert wurden und reagiert gegebenenfalls mit Korrekturmaßnahmen oder Planrevisionen. Ausgehend von diesem letzten Schritt schließt sich der Kreis im Managementprozess, indem neue Pläne und Zielvorgaben eingeleitet werden.

29 Vgl. hierzu ausführlich Schreyögg, G. (1991), S. 264-276. 


\subsubsection{Komplexität als modernes Managementproblem}

Wie einleitend dargestellt, definieren sich Unternehmen als soziale Systeme, deren Existenz erst durch die Komplexitätsdifferenz ${ }^{30}$ zwischen System und Umwelt möglich ist. In diesem Sinne liegt die zentrale Funktion der Systembildung in der Reduktion von Komplexität, da ein System nur durch eine geringere Komplexität als die seiner Umwelt zu identifizieren ist. ${ }^{31}$ Hierbei beschreibt die Systemgrenze die Trennung von geringerer System- und höherer Umweltkomplexität. Im System werden nur bestimmte Umweltausschnitte abgebildet, um die für das System nicht verarbeitbare Umweltkomplexität zu reduzieren und somit Überschaubarkeit und Orientierung herzustellen. ${ }^{32}$ „Durch Reduktion wird Orientierung in einer überwältigend komplexen Umwelt möglich." ${ }^{33}$

Die systemspezifische Auswahl bestimmter Umweltausschnitte impliziert jedoch nicht den gleichzeitigen Wegfall der Umweltkomplexität. Diese ist nach wie vor vorhanden, wird jedoch durch die Selektionsleistung vom System ausgeblendet. ${ }^{34}$ Welche Umweltausschnitte als relevant und welche als vernachlässigbar erachtet werden, unterliegt den spezifischen Selektionen des Systems. Die jeweiligen Selektionsleistungen können daher auch nicht den Anspruch auf Allgemeingültigkeit erheben, da die Relevanz von Umweltausschnitten nicht objektivierbar ist. ${ }^{35}$ LUHMANN spricht in diesem Zusammenhang von Kontingenz: Alles kann so sein kann wie gedacht, aber eben auch

30 Komplexität lässt sich anhand der Anzahl und Verschiedenartigkeit von Elementen und der Beziehungen zwischen den Elementen charakterisieren. Vgl. Luhmann, N. (1985), S. 45-51; Schreyögg, G. (1993), Sp. 4232-4247; Bronner, R. (1992), Sp. 1121-1130.

31 Vgl. Luhmann, N. (1985), S. 12-14.

32 Vgl. Wygoda, S. (2005), S. 71.

33 Steinmann, H./Schreyögg, G. (2005), S. 140.

34 „Alle Selektion setzt Einschränkungen (constraints) voraus.“ Luhmann, N. (1985), S. 57.

35 Ein prominentes Beispiel für die situative Anpassung des als relevant erachteten Marktes ist das Unternehmen Nokia, welches sich vom Gummihersteller zum Marktführer im Mobilfunkbereich entwickelt hat. 
nicht. ${ }^{36}$ Grundsätzlich ist die Umwelt(entwicklung) für ein System unbestimmt, wodurch zugleich die Adäquanz der getroffenen Selektion permanent in Frage gestellt werden muss. ${ }^{37}$ Die Bildung und Erhaltung von Systemen sind damit untrennbar mit Unsicherheit und Risiko verbunden: „Komplexität in dem angegebenen Sinne heißt Selektionszwang, Selektionszwang heißt Kontingenz, und Kontingenz heißt Risiko. “" ${ }^{38}$ Vollkommene Sicherheit würde bedeuten, dass die Systeme auf jeden Umweltzustand reagieren bzw. die Umwelt systemadäquat einrichten können. Die hierfür notwendige ,requiste variety ${ }^{39}$ fehlt den Systemen. ${ }^{40}$

In direktem Zusammenhang mit der Komplexitätsreduktion steht eine zweite Dimension, die durch die Selektionsleistung von Systemen Berücksichtigung finden muss - die Dynamik. Laut LuHMANN ist der Begriff ,Zeit' mit dem Vorgang der Selektion eng verwoben: „Der Zusammenhang von Komplexität und Selektion [...] ist keine Zustandsbeschreibung. Er impliziert bereits Zeit, er kommt nur durch Zeit und in der Zeit zustande. Zeit ist der Grund für den Selektionszwang in komplexen Systemen, denn wenn unendlich viel Zeit zur Verfügung stünde, könnte alles mit allem abgestimmt werden. ${ }^{\text {"11 }}$ Im Folgenden soll daher die Betrachtung auf die zeitliche Dimension und - damit verbunden - die Dynamik gelenkt werden. Gerade für die Untersuchung von Pfadabhängigkeiten ist der Faktor ,Zeit' unabdingbar, da Pfade per Definition Prozesscharakter aufweisen und nicht losgelöst von einer zeitlichen Perspektive betrachtet werden können.

36 Vgl. Luhmann, N. (1985), S. 47.

37 Vgl. Wygoda, S. (2005), S. 71f.

38 Luhmann, N. (1985), S. 47. In diesem Zusammenhang wird auf das Bild der ,nichttrivialen Maschine' verwiesen, welche unanalysierbar und unvoraussagbar ist. Vgl. Steinmann, H./Schreyögg, G. (2005), S. 137.

39 Vgl. hierzu Ashby, W.R. (1965), S. 202-218.

40 Vgl. Luhmann, N. (1985), S. 47.

41 Luhmann, N. (1985), S. 70. 


\subsubsection{Dynamik als modernes Managementproblem}

Die im vergangenen Kapitel erörterte Problematik der Komplexitätsverarbeitung erfährt durch die zeitliche Dimension eine weitere Verschärfung. Zum einen beansprucht Selektion Zeit, zum anderen ist auch die Umwelt keine statische Größe, sondern beweglich und ständiger Veränderung ausgesetzt. Der Komplexitätsdruck erzeugt einen Selektionszwang, wodurch Umweltdynamik zu einer kritischen Größe wird. ${ }^{42}$ Selektionen können zu einem bestimmten Zeitpunkt sinnvoll und relevant sein, später jedoch ihre Adäquanz einbüßen. Als Beispiel dient das sog. Ikarus-Paradox:43

Besonders erfolgreiche Unternehmen laufen Gefahr, ihren Erfolg nicht langfristig fortsetzen zu können. Ursächlich dafür ist die Schwierigkeit, die ursprünglich stimmigen Selektionen neu zu justieren und gegebenen Umweltveränderungen anzupassen. Die ehemals erfolgreiche Selektion und daraus abgeleitete Ausrichtung der Unternehmung behindern in der Konsequenz dessen nötige Flexibilität und Weiterentwicklungsmöglichkeiten. Eine zu starke Fokussierung auf etablierte Strategien und Strukturen birgt somit ein um so höheres Risikopotential.

Es lässt sich schließen, dass eine rein statische Betrachtung von Organisation und Management zu kurz greifen würde und grundlegende Problemfelder nicht zu fassen vermag. Selektionen, z. B. in Form von Unternehmensstrategien und die Bearbeitung als relevant erachteter Geschäftsfelder, können zu einem bestimmten Zeitpunkt sehr erfolgreich sein, sich jedoch zu einem späteren Zeitpunkt aufgrund von wesentlichen Umweltveränderungen als problematisch erweisen. An diese Problematik wird im Rahmen der Theoriediskussion zur Pfadabhängigkeit sowie im empirischen Fallbeispiel in der vorliegenden Arbeit angeknüpft, spielt doch die Schwierigkeit, einst erfolg-

42 Vgl. Steinmann, H./Schreyögg, G. (2005), S. 142. Dynamik impliziert Veränderung und lässt sich durch die Größen Häufigkeit, Stärke und Regelmäßigkeit der Veränderungen einordnen. Vgl. Kubicek, H./Thom, N. (1976), Sp. 4001.

43 Vgl. Miller, D. (1990). 
reiche Strategien zu ersetzen bzw. neu auszurichten in der Pfadanalyse eine entscheidende Rolle.

Durch die Integration der Konstrukte Komplexität und Dynamik muss der klassische Managementprozess reformuliert werden, eine logisch-lineare Ableitung der Managementfunktionen Organisation, Personal, Führung und Kontrolle aus der primären Funktion der Planung erscheint obsolet. In einem modernen Managementverständnis treten die einzelnen Funktionen gleichberechtigt nebeneinander, und jeder Managementaufgabe muss ein eigenständiges Steuerungspotential zugesprochen werden. ${ }^{44}$ Durch hohe Unsicherheit kann es so z. B. erforderlich sein, starre Planvorgaben durch flexible Organisationsstrukturen zu ersetzen, so dass strukturelle Bedingungen die strategischen Pläne beeinflussen. ${ }^{45}$

Ziel ist es dabei, dynamische und unsichere (Umwelt)Bedingungen durch einen kontinuierlichen Selektionsprozess abzufedern, um die langfristige Bestandserhaltung der Organisation zu sichern.

\section{Abbildung 2: Moderner Managementprozess}

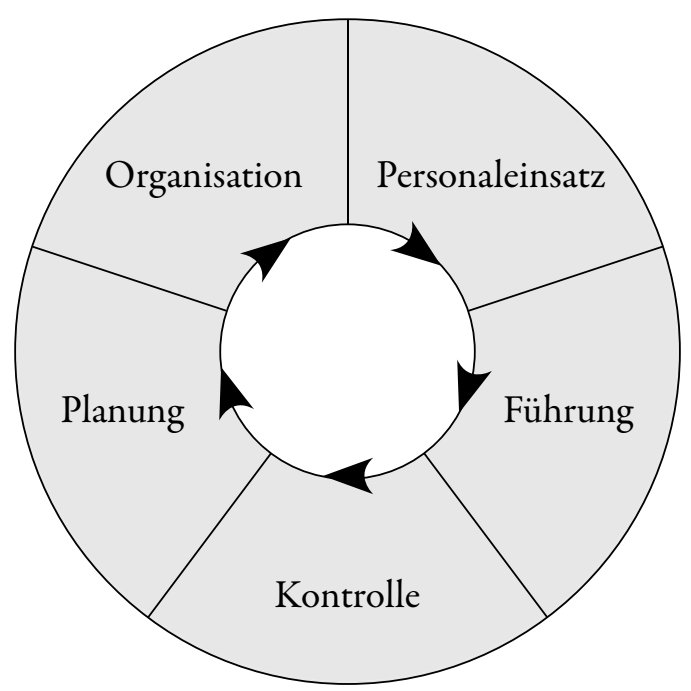

Quelle: Eigene Darstellung in Anlehnung an Steinmann, H./Schreyögg, G. (2005), S. 13.

44 Vgl. Steinmann, H./Schreyögg, G. (2005); S. 150.

45 Diese Bedingungen widersprechen den traditionellen Annahmen von ,structure follows strategy' Vgl. Chandler, A.D. (1962). 
Selektion versteht sich in dem beschriebenen Sinn nicht als freie Entscheidung, sondern unterliegt dem Zwang der Komplexitätsreduktion. ${ }^{46}$ Dass selektiert wird, lässt sich somit als Notwendigkeit auffassen; wie bzw. welche Umweltausschnitte als relevant erachtet und in welcher Form das weitere Handeln ausgerichtet wird, ist jedoch das Ergebnis von kontinuierlichen Entscheidungsprozessen.

Genau diese stehen im Zentrum der zu beantwortenden Forschungsfrage, gilt es doch den strategischen Entscheidungsverlauf des Bertelsmann Buchclubs zu analysieren, um Rückschlüsse auf eine potentielle Pfadabhängigkeit ziehen zu können. Für eine fundierte Analyse ist es daher zunächst notwendig, den für den Forschungsgegenstand zentralen Begriff der strategischen Entscheidungen definitorisch zu bestimmen.

\subsection{Strategische Entscheidungen: Theoretische Grundlagen}

Um den zentralen Begriff der strategischen Entscheidungen besser erschließen zu können, erfolgt zunächst eine Aufschlüsselung in die beiden Bestandteile ,Entscheidung' und ,Strategie’. Die Terminierung des Entscheidungsbegriffs und der idealtypischen Konzeption von Entscheidungsprozessen sollen insbesondere dabei helfen, die Trägheit von Entscheidungsverläufen im Rahmen der Pfadanalyse theoretisch zu fundieren, bevor auf die konkrete inhaltliche Bedeutung von strategischen Entscheidungsprozessen eingegangen werden kann. Ähnlich wie bereits bei den Grundlagen des Managements schließt das Kapitel mit den zentralen Prämissen der Strategietheorie, auf denen die Pfadanalyse in Kapitel 4 aufbaut.

\subsubsection{Zum Begriff der Entscheidung}

Entscheidungen liegen vor, sofern eine von mehreren, sich gegenseitig ausschließenden Handlungsmöglichkeiten ausgewählt wird. ${ }^{47}$ Der Entscheidungsbegriff basiert somit auf zwei grundlegenden, sich gegenseitig bedingenden Merkmalen: Der Wahl

46 Vgl. Steinmann, H./Schreyögg, G. (2005), S. 140.

47 Vgl. Witte, E. (1993), S. 910; Witte, E. (1992), S. 552; Sieben, G./Schildbach, T. (1994), S. 1; Wöhe, G. (2002), S. 120; Höschgen, H. (1992), S. $111 \mathrm{f}$. 
und dem Vorhandensein von Alternativen. Es liegt daher nur dann eine echte Entscheidungssituation vor, sofern mindestens zwei realisierbare Alternativen existieren, von denen unter Ausschluss der anderen die eine ausgewählt wird. ${ }^{48}$ Freie Entscheidungen setzen somit einen Entscheidungsspielraum voraus.

In Anlehnung an die Verhaltenswissenschaft werden Entscheidungen im Kontext eines Entscheidungsprozesses, der sich aus einzelnen Entscheidungsakten zusammensetzt, gesehen. ${ }^{49}$ Nachfolgende Abbildung 3 illustriert die einzelnen Schritte (Entscheidungsakte) des Entscheidungsprozesses.

Abbildung 3: Idealtypischer Entscheidungsprozess

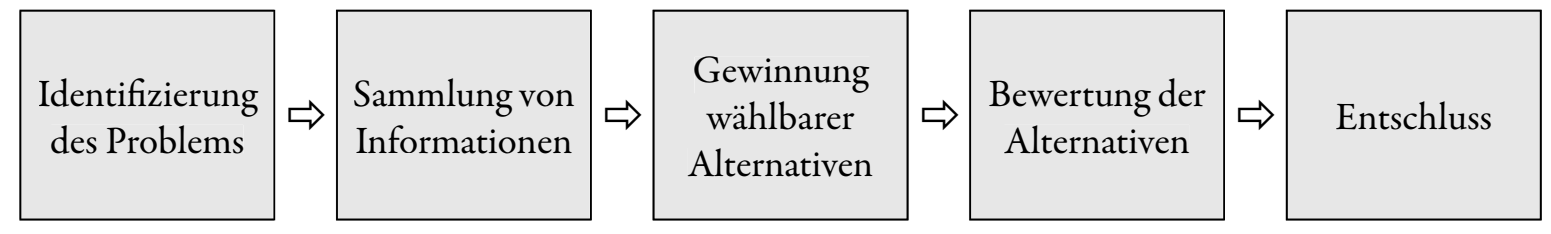

Quelle: Eigene Darstellung in Anlehnung an Witte, E. (1992), S. 554.

Nach der Problemfeststellung als erstem Schritt werden in einem zweiten Schritt Informationen gesammelt, um in einem dritten Schritt unterschiedliche Alternativen als Entscheidungsmöglichkeiten zu generieren. Diese werden in einem vierten Schritt bewertet und führen als Ergebnis des Entscheidungsprozesses zu einem Entschluss. Hierbei ist zu beachten, dass das beschriebene Modell des Entscheidungsprozesses eine idealtypische Konstruktion ist, anhand derer die einzelnen Stufen zur Entscheidungsfindung rein konzeptionell erläutert werden können..$^{50}$ Reale Entscheidungsprozesse

48 Im strengen Sinne bezieht sich der Begriff ,Alternative' lediglich auf eine, nämlich ,die andere' Handlungs- oder Entscheidungsmöglichkeit. In allgemeinen Sprachgebrauch hat sich ein weiteres Verständnis durchgesetzt, welches von Alternativen im Sinne von ,Varianten' ausgeht. Alternativen sowie Varianten bezeichnen mindestens eine Wahlmöglichkeit. Diesem Begriffsverständnis folgt auch die vorliegende Arbeit.

49 Vgl. Witte, E. (1992), S. 552-554.

50 Vgl. Höschgen, H. (1992), S. 114. 
sind dagegen schwer zu vereinheitlichen; einzelne Entscheidungsakte können einander überlagern, in umgekehrter Reihenfolge ablaufen oder gänzlich wegfallen.

Wichtig ist, dass - egal, welche Entscheidungsakte in welcher Reihenfolge den Prozess konstituieren - am Ende ein Entschluss steht, der als Ergebnis implementiert und als solcher beobachtbar ist. ${ }^{51}$ In diesem Sinne soll in der vorliegenden Arbeit von Entscheidungen als Entschlüssen oder auch punktuellen Akten gesprochen werden; wohl wissend, dass diese aus einem Entscheidungsprozess entstanden sind.

Nachdem Begriff und Entstehung von Entscheidungen geklärt sind, gilt es im nun folgenden Kapitel auf einen speziellen Typus von Entscheidungen einzugehen. Es handelt sich um strategische Entscheidungen, die den zentralen Forschungsgegenstand der vorliegenden Arbeit ausmachen.

\subsubsection{Zum Begriff der Strategie}

Der Terminus ,Strategie' - aus dem Griechischen von ,strategos' stammend - meint in seiner ursprünglichen Bedeutung ,die Kunst der Heerführung's2 und bezieht sich auf die Erstellung einer übergreifenden Gesamtkonzeption, um ein langfristiges Ziel zu erreichen. Seinen Einzug in die Wirtschaftswissenschaft hielt das Strategiekonzept erstmals in den 50er Jahren an der Harvard Business School. ${ }^{53}$ Seitdem genießt der Strategiebegriff in der Management- und Organisationsforschung bis heute uneingeschränkte Popularität, was die Festlegung einer klaren Definition für die vorliegende Arbeit unumgänglich macht. ${ }^{54}$

Die Pfadanalyse soll eine umfassende Untersuchung des strategischen Entscheidungsverlaufs im Bertelsmann Buchclub leisten. Hierfür lohnt sich eine Unterteilung in unterschiedliche Strategieebenen, anhand derer sich strategischer Wandel bzw.

51 Vgl. Witte, E. (1992), S. 553; Staehle, W.H. (1991), S. 485.

52 Vgl. Kreikebaum, H. (1993), S. $24 \mathrm{f}$.

53 Vgl. Snow, C.C./Hambrick, D.C. (1980), S. 527.

54 Für eine ausführliche Übersicht siehe Bracker, J. (1980), S. 219-224. 
Kontinuität möglichst vollständig erfassen lassen. Einen systematischen Überblick über die verschiedenen Facetten von Unternehmensstrategien liefern STEINMANN/SCHREYÖGG (2005), indem sie drei zentrale Bestandteile aus einer Vielzahl von Strategiekonzepten herausfiltern: die Festlegung der strategischen Geschäftsfelder, die Positionierung gegenüber den Wettbewerbern sowie die eigene Kompetenzbasis.

Während das letzte Feld, die Frage nach den Unternehmenskompetenzen, eine konzeptionelle Sonderposition einnimmt, ${ }^{55}$ verbleiben mit den ersten beiden Feldern zwei grundlegende Dimensionen von Unternehmensstrategien:

Zunächst werden im Rahmen der Gesamtunternehmensstrategie (Corporate Strategy) strategische Geschäftsfelder der Organisation bestimmt und entsprechende Ressourcen verteilt. Zentrale Frage ist hier, wo sich die Unternehmung positioniert, d. h. welche Produkt- und Marktsegmente bearbeitet werden. ${ }^{56}$ Die Wettbewerbsstrategie (Business Strategy) justiert dann die zentralen Aktionsparameter in den zuvor differenzierten Geschäftsfeldern. Grundlage bilden hierbei die unternehmensinternen und -externen Wettbewerbsbedingungen, welche je nach Geschäftsfeld sehr unterschiedlich ausfallen können. ${ }^{57}$

Mit den Ebenen der Corporate und Business Strategy können zwar grundlegende Bereiche der strategischen Ausrichtung von Unternehmen erfasst werden; für eine vollständige Analyse der Unternehmensstrategien fehlt jedoch der konzeptionelle Überbau, der das eigene strategische Selbstverständnis einer Unternehmung einfängt. Dies wird in der vorliegenden Arbeit mit der Integration der dritten, übergreifenden Ebene des Strategiebegriffs nachgeholt: dem Geschäftsmodell. ${ }^{58}$ In Abgrenzung zu den

55 Vgl. Steinmann, H./Schreyögg, G. (2005), S. 171.

56 Vgl. Hamel, G. (2000), S. 73; Steinmann, H./Schreyögg, G. (2005), S. 169.

57 Hofer, C.W./Schendel, D (1978), S. 27-29; Grant, R.M. (2002), S. 23-25.

58 Vgl. Grant, R.M./Nippa, M. (2006), S. 41 f. 
Termini ,Vision' und ,Leitbild'59 wird unter einem Geschäftsmodell die Geschäftsidee bzw. die hinter dem Geschäft stehende ökonomische Logik verstanden. Mit Hilfe des Geschäftsmodells soll die Frage beantwortet werden, „was [...] die Gewinn generierende Grundlage in [einem] Geschäft ist" ${ }^{60}$ Das Geschäftsmodell bildet somit die übergeordnete Ebene, aus der sich die Bestimmung der strategischen Geschäftsfelder (Corporate Strategy) und der Wettbewerbsstrategien (Busines Strategy) zur Umsetzung der Geschäftsidee ableitet. Die drei aufeinander aufbauenden Dimensionen dieses Strategieverständnisses sind in Abbildung 4 graphisch dargstellt.

Abbildung 4: Drei Ebenen des Strategiebegriffs

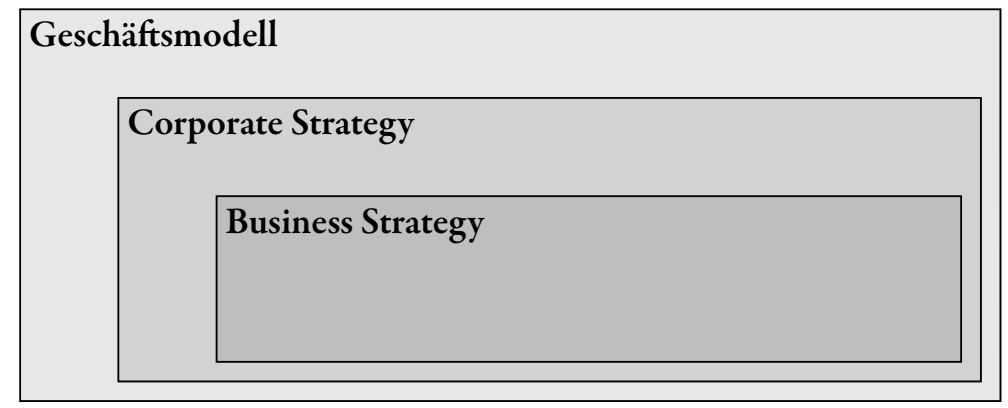

Quelle: Eigene Darstellung.

Die Aufgabe von Unternehmensstrategien ist, eine möglichst große Konsistenz (,Strategic Fit') zwischen Unternehmen und der externen Umwelt herzustellen. Zur nachhaltigen Erfolgssicherung müssen hierzu die Ziele, Ressourcen und Strukturen auf Unternehmensseite mit den Bedingungen des relevanten Marktes wie Wettbewerberund Kundenstruktur übereinstimmen. ${ }^{61}$ Denkbar ist hierbei nicht nur die strategische Anpassung des Unternehmens an herrschende Umweltbedingungen, sondern auch umgekehrt die aktive Einflussnahme und Veränderung derselben, z. B. durch die Ge-

59 Während der Begriff, Vision' auf die zukünftige Ausrichtung eines Unternehmens Bezug nimmt, richtet sich ein unternehmerisches, Leitbild' an Wertvorstellungen, Normen und Verhaltensmustern aus. Vgl. Hamel, G. (2000), S. 72f. sowie Steinmann, H./Schreyögg, G. (2005), S. 171; S. 715.

60 Grant, R.M./Nippa, M. (2006), S. 41.

${ }_{61}$ Vgl. Grant, R.M./Nippa, M. (2006), S. 34-36. 
nerierung neuer Marktsegmente. ${ }^{62}$ Das Bindeglied zwischen beiden Seiten - Unternehmung und Umwelt - bildet die Strategie, wie folgende Abbildung 5 verdeutlicht.

Abbildung 5: Strategie als Verbindung zwischen Unternehmen und Umwelt

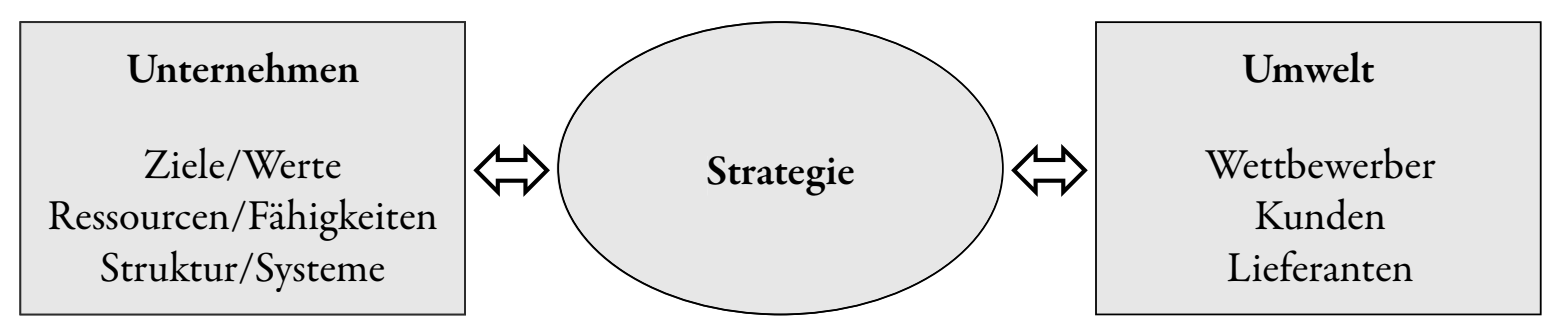

Quelle: Grant, R.M./Nippa, M. (2006), S. 34.

Abschließend lassen sich also unter Unternehmensstrategien die langfristig orientierten Entscheidungen verstehen, welche grundsätzliche Idee verfolgt wird, in welchen Branchen und Märkten eine Unternehmung agieren soll und welche Handlungsweisen und Ressourcenverwendungen zu wählen sind, um eine vorteilhafte Wettbewerbsposition zu erreichen. ${ }^{63}$

Anhand der definitorischen Abgrenzung des Strategiebegriffs wird bereits deutlich, dass in einem managementtheoretischen Kontext der Strategiebegriff eng, ja sogar untrennbar mit dem der Entscheidung verbunden ist. (Unternehmens)Strategien lassen sich - sofern eine Wahlmöglichkeit zwischen mindestens zwei unterschiedlichen Strategien besteht - als Gegenstand strategischer Entscheidungsprozesse auffassen. Eine bestimmte realisierte Unternehmensstrategie (z. B. Kostenführerschaft im Teilmarkt) ist somit ein beobachtbarer strategischer Entschluss als Ergebnis eines vorhergegangenen Entscheidungsprozesses. Auch wenn strategische Entscheidungen häufig mit Attributen wie langfristig und planvoll konnotiert werden, wird von einer exklusiven Anbindung an die Managementfunktion der Planung abgesehen. Entscheidungen, auch

\footnotetext{
62 Vgl. Steinmann, H./Schreyögg, G. (2005), S. 177.

63 Schreyögg, G. (1984), S. 5.
} 
strategische, können im gesamten Managementprozess entstehen und getroffen werden. ${ }^{64}$

Nachdem die Zusammenhänge von Entscheidung und Strategie deutlich geworden sind, soll im Folgenden auf die theoretischen Prämissen unternehmensstrategischer Entscheidungen eingegangen werden. Diese schließen an das in Kapitel 2.1.2 erörterte Managementverständnis an und sollen zeigen, vor welchen theoretischen Grundannahmen die Pfadanalyse zur Anwendung kommt.

\subsubsection{Strategietheoretische Grundlagen}

Das dieser Arbeit zugrunde liegende Strategieverständnis fußt auf den zentralen Annahmen der deskriptiven Strategietheorie, die das tatsächliche strategische Verhalten in und von Organisationen auf Muster und Gesetzmäßigkeiten hin untersucht. ${ }^{65}$ Werden - wie in der Fragestellung der vorliegenden Arbeit - empirische Entscheidungsverläufe analysiert, muss konsequenterweise von den präskriptiven Annahmen einer Individualrationalität und eines Planungsdeterminismus Abstand genommen werden. Obwohl planerisch entworfen, können praktische Entscheidungsverläufe durch Störfaktoren wie z. B. unvollkommene Information, Macht oder persönliche Emotionen beeinflusst werden und somit einen nicht $\mathrm{zu}$ antizipierenden Verlauf nehmen. Es können weder sämtliche Entscheidungsoptionen wahrgenommen, noch

64 Vgl. Schreyögg, G. (2005), S. 10. Vgl. hierzu die Ausführungen zur Strategietheorie im folgenden Kapitel 2.2.3.

65 Im Gegensatz zur deskriptiven Strategietheorie verfolgt der präskriptive Ansatz das Ziel, Gestaltungsanleitungen und Empfehlungen für das strategische Management auszusprechen. Entsprechend wird die präskriptive Strategie- oder auch Entscheidungstheorie ,normative Theorie' genannt. Neben den beiden Reinformen der deskriptiven und präskriptiven Theorie haben sich weitere Strategietheorien entwickelt, die beide Ansätze miteinander vereinen. So gibt es präskriptive Ansätze, deren Erkenntnisse aus Praxisbeobachtungen generiert werden und umgekehrt deskriptive Modelle, die Handlungsempfehlungen aus Beobachtungen ableiten. Vgl. Schreyögg, G. (1985), S. 133-138; Jungermann, H. (1976), S. 17f.; vgl. Becker, A. (1996), S. 8. 
deren Konsequenzen vollständig abgewogen und beurteilt werden, so dass (strategische) Entscheidungen prinzipiell unter begrenzter Rationalität getroffen werden. ${ }^{66}$

In diesem Sinne lassen sich Strategien zwar intentional planen, diese müssen jedoch nicht zwangsweise auch zur Realisation gelangen. Umgekehrt ist es ebenso möglich, dass sich Strategien emergent entwickeln. Für das strategische Management bedeuten diese Annahmen zwar die Unmöglichkeit einer vollständigen Planbarkeit, allerdings darf ebenso wenig absolute Steuerungslosigkeit unterstellt werden, ist es doch gerade der Anspruch des Managements, die Entwicklung einer Organisation intentional zu gestalten. Problematisch wird die intentionale Steuerung erst, sofern - wie im Falle der Pfadabhängigkeit - strategische Entscheidungsprozesse eine Eigendynamik entwickeln, auf die das Management nur noch bedingt Einfluss nehmen kann. Eine ausführliche Diskussion zu dieser Dynamik und den managementtheoretischen Implikationen folgt in Kapitel 2.3 zur Theorie der Pfadabhängigkeit.

Tabelle 1 fasst die beschriebene Unterscheidung nach intendierten und emergenten sowie realisierten und nicht realisierten Strategien in einer Vier-Felder Matrix graphisch zusammen. Die Matrix zeigt, dass logisch vier, praktisch relevant jedoch nur drei Typen von Unternehmensstrategien möglich sind: nicht realisierte, beabsichtigte und emergente Strategien. Strategien sind nach diesem Schema daher keinesfalls ausschließlich Ergebnis einer intendierten, planvollen Gestaltung, sondern können sich selbständig, plötzlich und permanent im zeitlichen Verlauf entwickeln. Denkbar sind auch Mischformen, so dass sich eine einst absichtsvoll geplante Strategie emergent weiterentwickelt und zum Selbstläufer wird.

66 Zum Begriff ,bounded rationality' vgl. ausführlich March, J.G./Simon, H.A. (1958), S. 136171; vgl. Jungermann, H. (1976), S. 36-39. 
Tabelle 1: Klassifikationsschema für Unternehmensstrategien

\begin{tabular}{l|c|c} 
Strategien & nicht realisiert & realisiert \\
\hline intendiert & $\begin{array}{c}\text { "Unrealized Strategy“ } \\
\text { (intendiert, aber nicht realisiert) }\end{array}$ & $\begin{array}{c}\text { "Deliberate Strategy“ } \\
\text { (intendiert und realisiert) }\end{array}$ \\
\hline emergent & $(\mathrm{X})$ & $\begin{array}{c}\text { „Emergent Strategy“ } \\
\text { (realisiert, aber nicht intendiert) }\end{array}$ \\
\hline
\end{tabular}

Quelle: Eigene Darstellung in Anlehnung an Mintzberg, H. (1977), S. 945; Schreyögg, G. (1985), S. 148.

\subsubsection{Zwischenfazit}

Auf Basis der getroffenen Aussagen werden Unternehmen aus einer systemtheoretischen Perspektive als soziale Handlungssysteme aufgefasst. Die Steuerung der Unternehmung wird durch die Managementfunktionen Planung, Organisation, Personaleinsatz, Führung und Kontrolle vollzogen, wobei jeder Funktion autonomes Steuerungspotential zugeschrieben wird. Strategische Entscheidungen - wenn auch konzeptionell eng mit der Planungsfunktion verbunden - sind im Kontext des gesamten Managementsystems zu sehen. Hierbei impliziert eine Entscheidung die unter begrenzter Rationalität getätigte Auswahl einer unter mehreren Handlungsoptionen. Unternehmensstrategien als Gegenstand bzw. Ergebnis strategischer Entscheidungsprozesse beziehen sich auf die gesamte Organisation und / oder einzelne Geschäftsfelder im Sinne einer Wettbewerbsstrategie. Grundsätzlich können (nicht) realisierte Strategien sowohl planvoll entworfen als auch emergent gewachsen sein.

Auf diesem Grundverständnis von strategischen Entscheidungen in Organisationen baut im nun folgenden Kapitel die Diskussion zur Theorie der Pfadabhängigkeit auf. Unter Vorgriff auf die detaillierten pfadtheoretischen Ausführungen sowie die empirische Pfadanalyse in Kapitel 4 sei an dieser Stelle bereits angemerkt, dass gerade 
die Emergenz von strategischen Entscheidungsprozessen, die sich zunehmend einer strategischen Steuerung entziehen, von großer Bedeutung ist.

\subsection{Theorie der Pfadabhängigkeit}

Auch wenn der Begriff 'Pfad' bereits auf eine lange Tradition in der Wirtschaftswissenschaft zurückblickt, ${ }^{67}$ so besteht dennoch Einigkeit darüber, den eigentlichen Ursprung der ökonomisch motivierten Pfadtheorie mit den zwei zentralen Artikeln von DAVID (1985) und ARTHuR (1989) zu erfassen. ${ }^{68}$ Die Pfadtheorie fordert die neoklassische Annahme von langfristig effizienten Marktgleichgewichten heraus, indem sie das Phänomen eines Lock-Ins benennt - einen Zustand, in dem sich unter bestimmten Bedingungen ein ineffizientes, nicht reversibles Marktergebnis einstellt.

\subsubsection{Ursprung und konzeptionelle Öffnung der Pfadtheorie}

Beispielhaft beschreibt DAVID (1985) die emergente Entstehung eines technologischen Pfades anhand der Marktdurchsetzung der Schreibmaschinentastatur ,QWERTY' ${ }^{69}$ Als 1867 das Patent für die erste mechanische Schreibmaschine verliehen wurde, spielten bei der Anordnung der Tastatur vor allem zwei Faktoren eine wesentliche Rolle: Zum einen sollten sich die einzelnen Typenblätter beim Bedienen der Schreibmaschine möglichst selten verhaken, zum anderen galt es als förderlich, sofern die Schreibmaschinenvertreter in Verkaufsgesprächen das Wort ,typewriter' eindrucksvoll zu Papier bringen konnten. Dieses setzte sich daher ausschließlich aus der ersten Tastaturzeile zusammen. Obwohl die mechanische Schreibmaschine zunächst durch elektrische Varianten und später vollständig durch Computer abgelöst wurde und bereits mehrfach

67 Bereits SCHUMPETER führte wirtschaftliche Entwicklungen unter anderem auf die von zunehmender Erfahrung profitierenden Nachfolger der ersten Entrepreneure zurück und beschrieb einen ,Innovationspfad'. Vgl. Schumpeter, J.A. (1939), S. 131.

68 Vgl. Lawson, T. (1997), S. 247-271; Hultén, S. (2003), S. 114-117.

69 Vgl. David, P.A. (1985), S. 333. 
Tastaturen erfunden wurden, die ein bis zu $40 \%$ schnelleres Schreiben ermöglichen können, ${ }^{70}$ hat sich bis heute die QWERTY-Tastatur als marktführender Standard durchgesetzt. Ursächlich hierfür sind sog. positive Rückkopplungseffekte, welche die Etablierung des QWERTY-Standards bei gleichzeitiger Verdrängung von Alternativen bewirkten.

Das Beispiel macht vor allem drei zentrale Eigenschaften pfadabhängiger Prozesse deutlich: Non-Ergodizität, Irreversibilität und potentielle Ineffizienz. Unter NonErgodizität lässt sich die Möglichkeit unterschiedlicher Marktergebnisse fassen, da der Ausgang pfadabhängiger Prozesse per se nicht antizipierbar ist. ${ }^{71}$ Scheinbar unbedeutende, zufällige Ereignisse können einen Pfadprozess auslösen, dessen Ausgang jedoch zunächst noch unbestimmt ist. Bifurkationspunkte im Prozessverlauf beschreiben Punkte, an denen unterschiedliche Richtungen eingeschlagen werden können. Gemäß den pfadtheoretischen Annahmen wird ein Richtungswechsel allerdings mit fortschreitender Entwicklungsstufe zunehmend unwahrscheinlicher, weil der Mechanismus der positiven Rückkopplung vergangene Entscheidungen verstärkt und somit die Durchsetzung eines Standards wie der QWERTY-Tastatur bewirkt. Pfadverläufe sind somit irreversibel, d. h. ein sich herausbildender Pfad kann nur schwerlich verlassen werden, selbst, wenn sich das Ergebnis als einer Alternative gegenüber unterlegen erweist. Hierfür ist der Zustand des Lock-Ins als Resultat pfadabhängiger Prozesse ursächlich.

Wie bereits zu Beginn dieses Kapitels erwähnt, genießt der Begriff der Pfadabhängigkeit seit einiger Zeit starke Popularität. Neben gesellschaftspolitischen Phänomenen wie Reformstau, wirtschaftsgeographischen Entwicklungen und Biographieforschung werden mit Hilfe der Pfadtheorie die Verbreitung von technologischen Standards im Rahmen der Innovationsforschung, aber auch Strategieprozesse auf organisa-

\footnotetext{
$70 \quad$ Vgl. David, P.A. (1985), S. 332.
}

71 Vgl. Ackermann, R. (2003), S. 229. 
tionaler Ebene analysiert. ${ }^{72}$ Gerade durch die sich auf den ersten Blick erschließende Eingängigkeit der pfadtheoretischen Grundannahmen sowie der bildlichen Analogie des spontane Assoziationen auslösenden ,Pfades' werden häufig ganz allgemein historisch gewachsene Prozesse, Momente von Trägheit und Irreversibilität mit Pfadabhängigkeiten gleichgesetzt. Aufgrund der vielfältigen Anwendung und Übertragung der Pfadtheorie auf sehr unterschiedliche wissenschaftliche Disziplinen wird der Pfadabhängigkeit auch ein breites Spektrum von teils divergierenden notwendigen sowie hinreichenden Bedingungen zugeschrieben. Diese hohe Ausprägungsvielfalt impliziert logischerweise eine unterschiedliche Qualität von Pfadabhängigkeiten, welche sich neben der reinen Identifizierung von Pfadphänomenen auch auf die Behandlung von Wandel, Pfadbruch und generellen Gestaltungsmöglichkeiten niederschlägt. Aus diesen Gründen erscheint eine kurze Übersicht über die wesentlichen Strömungen in der pfadtheoretischen Debatte unvermeidbar. Bei der Darstellung der einzelnen Ansätze wird auf einen Bezug zur Ebene der Organisation geachtet, um die theoretischen Konzepte für die empirische Analyse im Rahmen der Fallstudie fruchtbar zu machen. ${ }^{73}$

Sowohl DAVID (1985) als auch ARTHUR (1989) betrachten Pfadabhängigkeiten aus einer ökonomisch geprägten Perspektive und betonen die Notwendigkeit von Increasing Returns als zentralen Mechanismus der Selbstverstärkung zur Pfadidentifikation. ${ }^{74}$ Hierbei nennt ARTHUR vier sich selbst verstärkende Effekte, die einen pfadabhängigen Verlauf nach sich ziehen können: Start- bzw. Fixkostenintensität als Voraussetzung für Degressionseffekte, Lerneffekte, Kooperationseffekte sowie adaptive Erwartungen, über die der Zusammenhang zwischen aktueller Verbreitung und zukünftiger Nutzung hergestellt wird.

72 Vgl. Beyer, J. (2005), S. 6 ; Sydow et al. (2005), S. 3f. ; Gartland, M.P. (2005); sowie Guinnane, T. et al. (2003), Mahoney, J. (2000), Pierson, P. (2000),

73 Für eine ausführlichere Übersicht siehe Beyer, J. (2006), S. 11-20; Beyer, J. (2005), S. 5-23; Dievernich, F.E.P. (2007), S. 12-28; Thelen, K. (1999), S. 369-404.

74 Vgl. David, P.A. (1985), S. 332-337; Arthur, W.B. (1989), S. 116-131. 
DAVID bezieht sich bei der Analyse der QWERTY-Diffusion auf drei zentrale Ursachen mit selbstverstärkendem Charakter. Alle drei Faktoren werden in einem kombinatorischen Wirkungszusammenhang gesehen: Steigende Skalenerträge, technische Verknüpfungen als Zusammenspiel von technologischer Verbreitung und gelerntem Erfahrungswissen sowie Quasi-Irreversibilitäten von Investitionen im Sinne von versunkenen Kosten (Sunk Costs).

Im Gegensatz zu dem von DAVID und ARTHUR getroffenen Konsens, Increasing Returns als notwendige Bedingung für Pfadabhängigkeit $\mathrm{zu}$ sehen, erweitert ARrow (2003) $)^{75}$ das bestehende Pfadkonzept um Constant Returns, also konstante Erträge. Für ARROW sind nicht steigende Skalenerträge, sondern ist ausschließlich die Quasi-Irreversibilität von Investitionen der kritische Faktor einer Pfadabhängigkeit. Gestützt auf die Ergebnisse empirischer Studien können sich demzufolge pfadabhängige Prozesse neben der Voraussetzung von steigenden Skalenerträgen auch bei vollkommenem Wettbewerb und unter konstanten Erträgen herausbilden.

Eine weitergehende Ausdehnung der Pfadtheorie wird durch MAHONEY (2000) ${ }^{76}$ vorgenommen. Zunächst unterscheidet MAHONEY zwei unterschiedliche Typen von Pfadabhängigkeit: Pfadabhängigkeiten, die auf Selbstverstärkung beruhen und Pfadabhängigkeiten auf Basis reaktiver Sequenzen (,Reactive Sequences'). Während sich die Form der selbstverstärkenden Pfadabhängigkeit im Grundsatz an die von DAVID und ARTHUR beschriebenen Prozesse anlehnt, wird mit der Einführung von reaktiven Sequenzen eine gänzlich neue Form von Pfadabhängigkeit beschrieben.

Unter reaktiven Sequenzen wird eine zeitliche Abfolge kausal verknüpfter Ereignisse verstanden, so dass frühe Ereignisse das potentielle Prozessergebnis beeinflus-

75 Vgl. Arrow, K.J. (2003), S. 23-35.

76 Vgl. Mahoney, J. (2000), S. 507-548. 
sen. ${ }^{77}$ Wichtig ist, und hier liegt auch ein wesentlicher Unterschied zur selbstverstärkenden Pfadabhängigkeit, dass ein gegebenes Muster, eine Entscheidung nicht notwendigerweise verstärkt wird, sondern als Auslöser für die folgende Kausalkette gilt. Aktion und Reaktion können das System somit in eine neue Richtung bewegen.

Auch die von MAHONEY beschriebene selbstverstärkende Pfadabhängigkeit muss differenziert betrachtet werden, da sich die Grundannahme der selbstverstärkenden Prozesse zwar mit den klassischen Pfadbedingungen deckt, jedoch durch die Angabe von vier unterschiedlichen Reproduktionsmechanismen entscheidend modifiziert wird. Ein erster Reproduktionsmechanismus basiert auf utilitaristischen Gründen, indem die (rationalen) Entscheidungen von Akteuren zur institutionellen Stabilität führen. Hierbei können adaptive Erwartungen - wie bereits bei ARTHUR erwähnt sowie Koordinationseffekte zu einer Verfestigung ineffizienter Muster beitragen. Funktionalistische Gründe beruhen auf der Funktionserfüllung einer Organisation innerhalb eines Gesamtsystems. Ineffizienzen können sich herausbilden, sofern eine potentiell schlechtere Funktionalität im Gegensatz zu vorherigen Alternativen vorliegt. Als dritte Ursache für Pfadabhängigkeiten werden elitäre Machtstrukturen angeführt. In einem sukzessiven, politisch motivierten Prozess kann es einem Teilsystem gelingen, Machtpotential zu generieren und entscheidenden Einfluss innerhalb der Organisation zu gewinnen. Das Entscheidungsverhalten des machtvoll elitären Subsystems kann so zu einer Reproduktion von für die Gesamtorganisation ineffizienten Entscheidungsmustern führen. Schließlich bildet die Legitimation den letzen selbstverstärkenden Mechanismus zur Pfadabhängigkeit. Moralische Verpflichtungen sowie persönliche Wertvorstellungen und Empfindungen beteiligter Akteure können das

77 „Reactive sequences are chains of temporally ordered and causally connected events. These sequences are 'reactive' in the sense that each event within the sequence is in part a reaction to temporally antecedent events. Thus, each step in the chain is 'dependent' on prior steps." Mahoney, J. (2000), S. 509. 
Handeln beeinflussen und für Entscheidungsreproduktionen und die Herausbildung von Pfadabhängigkeiten ursächlich sein. ${ }^{78}$

Wie diese vorgestellten Ansätze von ARROW und MAHONEY bereits andeuten, ist die eher klassische Pfadtheorie von DAVID und ARTHUR - nicht zuletzt aufgrund der Übertragung in unterschiedliche Wissenschaftsbereiche - modifiziert und erweitert worden.

PIERSON (2000) weist in diesem Zusammenhang auf die inflationäre Anwendung und somit die Gefahr der Überdehnung der Pfadtheorie hin. ${ }^{79}$ Um einer konzeptionellen Verwässerung vorzubeugen, empfiehlt sich eine begriffliche Aufspaltung in zwei unterschiedliche Pfadverständnisse: Pfadabhängigkeit im weiteren und engeren Sinne. Ein weit gefasstes Pfadverständnis bezieht sich im Kern auf die Historizität von Prozessen (,history matters'), so dass späte Ereignisse und Entscheidungen aus einer historischen Prozessperspektive betrachtet und analysiert werden können. Ein aktueller $\mathrm{Zu}$ stand lässt sich somit auf die Sequenz vorhergehender Ereignisse zurückführen. ${ }^{80}$

Historizität und eine dynamische Prozessperspektive vermögen sicherlich gegenüber rein statischen Analysen einen beachtlichen Erkenntnisgewinn generieren; dennoch muss die Frage nach Originarität und Erklärungskraft des ,history matters'Ansatzes gestellt werden. Bereits seit den 80er Jahren wird in der Managementliteratur beschrieben, dass der Kontext früher Entscheidungen die spätere Entscheidungsfindung und Strategieentwicklung beeinflusst. ${ }^{81}$ Aktuelle Strategien werden somit nicht isoliert, sondern vor dem Hintergrund ihrer strukturellen Verankerung und des vorangegangenen Entscheidungsverlaufs betrachtet. Eine Bewertung sämtlicher histo-

78 Vgl. Mahoney, J. (2000), S. 517-524.

79 „Analysts are increasingly inclined to invoke the concept of path dependence, but clear definitions are rare. [...] Path dependence has been a victim of what Sartori (1970) called concept stretching." Pierson, P. (2000), S. 252. Neben der Gefahr spricht Pierson der Ausweitung des Pfadkonzepts jedoch auch großes Potential zu.

80 Vgl. Pierson, P. (2000), S. $251 \mathrm{f}$.

81 Vgl. Hall, D.J./Saias, M.A. (1980), S. 149-163. 
risch verankerter (Entscheidungs-)verläufe und Prozessketten als pfadabhängig muss sich somit mit dem Vorwurf von Beliebigkeit auseinandersetzen. Der zentrale Erklärungsgehalt der Pfadtheorie, auf den im Folgenden noch ausführlich eingegangen werden wird, kann in diesem weiten Begriffsverständnis nur bedingt greifen, gerade weil sämtliche historisch geprägten Prozesse als pfadabhängig gelten könnten.

In diesem Sinne stützt sich die vorliegende Arbeit auf ein enges Pfadverständnis, deren zentrale Annahmen im folgenden Kapitel ausführlich erörtert werden.

\subsubsection{Fokussierung: Pfadabhängigkeit im engeren Sinne}

Das Konzept der Pfadabhängigkeit im engeren Sinne fußt auf drei zentralen Säulen, die zugleich in chronologischer Abfolge die einzelnen Phasen im Pfadentstehungsprozess beschreiben: Historizität, der Mechanismus der positiven Rückkopplung sowie der Lock-In. Alle drei Säulen werden in den folgenden Kapiteln detailliert erläutert.

\subsubsection{Historizität}

Die erste Säule des engen Pfadverständnisses wird durch die zentrale Annahme der Historizität des weiten Konzepts beschrieben. Während das ,history matters'Argument in einer weiten Auslegung notwendige und zugleich hinreichende Bedingung für einen pfadabhängigen Prozess beschreibt, bildet die strukturell-historische Einbettung strategischer Entscheidungen in einer engen Auslegung zwar einen notwendigen, jedoch nicht ausreichenden Bestandteil eines pfadabhängigen Prozesses. Auch wenn die Pfadtheorie idealtypisch von einer anfänglichen Kontingenz ${ }^{82}$, d. h. einem nahezu unbegrenzten Entscheidungsspielraum ausgeht, sind gerade in einem Setting von Organisationen als soziale Systeme strategische Entscheidungsprozesse

82 Kontingenz meint, „dass historische Entwicklungen offen sind und nicht in irgendeinem Sinn ex ante festgelegt." Ackermann, R. (2001), S. 2f. Die Unmöglichkeit, den Eintritt von Ereignissen zu antizipieren, beschreibt auch MAHONEY, indem er Ereignisse als kontingent bezeichnet, „when its explanation appears to fall outside of existing scientific theory." Mahoney, J. (2000), S. 514. 
nicht losgelöst von ihrem organisationalen Kontext zu sehen. ${ }^{83}$ Prägnante Beispiele für prägende Kontextfaktoren können bestehende Organisationsstrukturen, aber auch die machtvolle Einflussnahme durch Eigentümer, Unternehmensgründer sowie Stakeholder sein.

Festzuhalten bleibt, dass zu Beginn eines sich herausbildenden Pfades von ,eingeschränkter Kontingenz' auszugehen ist, da der Handlungsspielraum bereits durch Kontextfaktoren beeinflusst ist. Der bestehende Kontext kann zwar auf den Pfadverlauf einwirken, Form und Richtung der potentiellen Entscheidungstrajektorie sind in dieser Frühphase jedoch noch offen und undeterminiert. Diese anfängliche Ungerichtetheit pfadabhängiger Prozesse wird im weiteren Verlauf auf Basis von Selbstverstärkungsmechanismen zunehmend eingeengt. Als Auslöser gilt ein Critical Juncture, ein kritisches Ereignis, das zum Zeitpunkt des Eintretens häufig als solches nur schwer zu erkennen ist, da es auf kleinen Ereignissen (Small Events) beruht, die allerdings im anschließenden Prozessverlauf durch die Initiierung des positiven Rückkopplungsmechanismus große Wirkung zeigen. Auf diese zweite Phase des Pfadentstehungsprozesses, die Phase der positiven Rückkopplung, wird im folgenden Kapitel detailliert eingegangen.

\subsubsection{Der Mechanismus der positiven Rückkopplung}

Zum besseren Verständnis der Wirkweise von positiver Rückkopplung soll zunächst der Begriff des Mechanismus genauer erläutert werden, um im Anschluss daran die einzelnen Formen von Selbstverstärkungsmechanismen ausführlich vorzustellen.

\subsection{Zum Begriff des Mechanismus}

Um die Wirkungsweise der positiven Rückkopplung besser erschließen zu können, lohnt es sich zunächst, den Begriff des Mechanismus genauer zu durchleuchten.

83 Vgl. Teece, D.J. (1997), S. 522f.; Schreyögg, G. et al. (2003), S. $271 \mathrm{f}$. 
Der Terminus ,Mechanismus' stammt ursprünglich aus der Technologie und bezeichnet „gekoppelte Bauelemente (einer Maschine, einer technischen Vorrichtung, eines technischen Geräts, Instruments o. ä.), die so konstruiert sind, dass jede Bewegung eines Elements eine Bewegung anderer Elemente bewirkt. ${ }^{\text {84 }}$ Mit dieser Definition sind bereits zwei zentrale Eigenschaften von Mechanismen benannt.

Zunächst lässt sich durch einen Mechanismus das Zusammenspiel mehrerer Elemente beschreiben. Sofern bestimmte Anfangsbedingungen gegeben sind, lässt sich durch den Mechanismus die Konsequenz, das Ergebnis, aus den Startkonditionen ableiten. Implizit ist somit bereits die zweite Eigenschaft von Mechanismen formuliert. Mechanismen beschreiben wiederholt auftretende Prozesse, so dass - technologisch gesprochen - eine Hebelbewegung A durch den Mechanismus stets ein Ergebnis B (z. B. der Antrieb eines Zahnrades) zur Folge hat.

Dieses maschinell geprägte Verständnis von Mechanismen muss zur Übertragung auf soziale Phänomene verallgemeinert werden, so dass als Mechanismen „wiederkehrende Prozesse, die bestimmte Ausgangsbedingungen mit einem bestimmten Ergebnis verknüpfen" ${ }^{\text {"85 }} \mathrm{zu}$ verstehen sind. Zentraler Gedanke ist dabei die Unterstellung von Kausalität. Mit Hilfe des Mechanismus lässt sich feststellen, wie die einzelnen Schritte einer Prozesskette logisch miteinander verknüpft sind. Somit wird - anders als bei der bloßen Bestimmung von Korrelationen und statistischen Zusammenhängen - der Fokus auf den Modus der einzelnen Verbindungen gelegt. In diesem Sinne erklärt ein Mechanismus nicht nur, dass bestimmte Korrelationen bestehen, sondern weiß auch, wie die Zusammenhänge zueinander in Bezug stehen. ${ }^{86}$ Wichtig ist hierbei, dass die

84 Duden (1989), S. 1000.

85 Mayntz, R. (2005), S. 207.

86 „Es reicht beispielsweise nicht aus, festzustellen, dass Ideen usw. Handeln beeinflussen; eine erklärende Theorie erfordert vielmehr ,a plausible mechanism to account for how symbols, traditions, rituals, and myths influence social and political interaction.'" Manytz, R. (2005), S. 208; Johnson, J. (2002), S. 227. 
Kausalstruktur von Mechanismen sowohl linear als auch nicht linear sein kann. ${ }^{87}$ Die einzelnen Elemente (z. B. Entscheidungen) einer Prozesskette können im Sinne einer Kettenreaktion nur einmal im gesamten Entwicklungsverlauf auftreten, sich aber auch wiederholen und rekursiv oder gegenseitig verstärkend aufeinander beziehen.

Abbildung 6 veranschaulicht idealtypisch drei zu unterscheidende Arten von durch einen Mechanismus verknüpften Kausalketten. ${ }^{88}$ Im ersten Fall, der linearen Verknüpfung, führt Element A zu Element B, ohne auf den Ausgang der Prozesskette rückzuwirken. Der zweite Fall beschreibt eine zirkulär-rekursive Rückkopplung, so dass sich ein Kreislauf zwischen den Elementen A und B ergibt. Schließlich impliziert der dritte Fall der zirkulär-verstärkenden Verknüpfung eine Spiralwirkung, die sich aus dem Mechanismus der positiven Rückkopplung ergibt. Element A führt zu einer Verstärkung von Element B, das wiederum rückverstärkend auf Element A einwirkt.

Abbildung 6: Wirkrichtungen kausaler Prozessketten

\begin{tabular}{c|c} 
Kausalkette & Art der Kausalität \\
\hline A $\Rightarrow$ B & linear \\
\hline A $\mathrm{A}$ \\
B $\diamond$
\end{tabular}

Quelle: Eigene Darstellung.

87 Vgl. Mayntz, R. (2005), S. 209.

88 Aus Gründen der Übersichtlichkeit bezieht sich die Übersicht bewusst auf die Wirkrichtungen von Mechanismen zwischen zwei Elementen A und B. Monge (1995) wählt eine andere Kategorisierung und unterteilt nach ,self-loop', ,mutual causal loop' sowie ,traditional feedback loop'. Vgl. Monge, P.A. (1995), S. 285-288. 
Wichtig ist hierbei, dass Mechanismen zwischen Elementen desselben Systems, allerdings auch zwischen Systemelementen und Elementen der Umwelt wirken können.

Der letzte Fall von kausaler Verknüpfung - der Mechanismus positiver Rückkopplung oder auch Selbstverstärkungsmechanismus beschreibt einen zentralen Faktor von pfadabhängigen Prozessen. Ein Selbstverstärkungsmechanismus wirkt durch das Vorliegen selbstverstärkender Effekte, auf deren Basis gehandelt wird ${ }^{89}$ Beispielsweise steigert sich die Attraktivität, eine bestimmte Entscheidung zu treffen, durch das Treffen gerade dieser Entscheidung, wodurch zunehmend diese eine Entscheidung getroffen wird. Welche unterschiedlichen Formen von Selbstverstärkungsmechanismen existieren und auftreten können, wird im nun folgenden Kapitel diskutiert.

\subsection{Selbstverstärkende Effekte und positive Rückkopplung}

Die klassische Pfadtheorie benennt als zentralen Selbstverstärkungsmechanismus 'Increasing Returns', also steigende Skalenerträge: ${ }^{90}$ „Increasing returns are the tendency for that which is ahead to go farther ahead, for that which loses advantage, to lose farther advantage." ${ }^{91}$ Die Zunahme bzw. Abnahme eines bestimmten Faktors bewirkt also eine steigende Wahrscheinlichkeit der weiteren Zunahme bzw. Abnahme desselben Faktors mit dem Ergebnis einer sich selbst verstärkenden Spiralwirkung.

Lässt sich diese Wirkung im mathematischen Modell der Polya-Urne ${ }^{92}$ noch klar messen, ist eine Übertragung der Increasing Returns in einen organisationalen Kontext aus zwei Gründen problematisch. Wie bereits einleitend dargestellt, werden strategische Entscheidungen häufig unter begrenzter Rationalität getroffen, so dass sich die dem Konzept der Increasing Returns zugrunde liegenden Prämissen der rationalen

89 Vgl. Koch, J. (2007a), S. 286.

90 Vgl. David, P.A. (1985); Arthur, B.W. (1989) sowie Kapitel 2.3.1 dieser Arbeit.

91 Arthur, B.W. (1996), S. 100.

92 Vgl. Arthur, B.W. (1989). 
Wahl und individueller Nutzenkalküle nicht durchhalten lassen. ${ }^{93}$ Zum zweiten lassen sich strategische und organisationale Entscheidungsverläufe nur schwer skalieren und messen, um eindeutige Aussagen zu steigenden Skalenerträgen treffen zu können.

Beispielhaft sei an dieser Stelle auf kognitive pfadabhängige Prozesse verwiesen, deren Selbstverstärkung im Sinne von Increasing Returns kaum metrisierbar ist. ${ }^{94}$ In Anlehnung an SCHREYÖGG ET AL. (2003) empfiehlt sich folglich eine Ausdehnung der nutzentheoretisch orientierten Increasing Returns auf einen allgemeinen Mechanismus positiver Selbstverstärkung. Diese Erweiterung der Increasing ReturnsBedingung darf nicht mit der in Kapitel 2.3.1 beschriebenen weiten Auslegung des Pfadverständnisses verwechselt werden, stellt sie doch, wie erläutert, eine forschungspragmatische Notwendigkeit bei der Betrachtung organisationaler und strategischer Pfade dar.

In der einschlägigen Literatur ist bereits ein breites Spektrum an unterschiedlichen Formen von Selbstverstärkungsmechanismen zusammengetragen worden. ${ }^{95}$ Hierbei wird grob zwischen technologisch und institutionell geprägten Formen unterschieden.

Folgende Tabelle 2 benennt die wesentlichen Ausprägungsformen von Selbstverstärkungsmechanismen. Erneut ist zu betonen, dass der Mechanismus der Selbstverstärkung erst durch das akteursgeleitete Handeln als Aktion und Reaktion auf selbstverstärkende Effekte zum Tragen kommt. ${ }^{96}$

93 Vgl. Schreyögg, G. et al. (2003), S. 266.

94 Vgl. Schreyögg, G. et al. (2003), S. 269.

95 Vgl. hierzu Beyer, J. (2006); Sydow, J. et al. (2005); Dievernich, F.E.P. (2007).

96 Vgl. Koch, J. (2007), S. 6. 
Tabelle 2: Formen von Selbstverstärkungsmechanismen

\begin{tabular}{l|l|l} 
& Form des Mechanismus & Kontext \\
\hline 1 & Economies of Scale/Economies of Scope & Technologie \\
\hline 2 & Direkte/indirekte Netzexternalitäten & Technologie \\
\hline 3 & Lernen & Technologie/Institution \\
\hline 4 & $\begin{array}{l}\text { Adaptive Erwartungen/ } \\
\text { Erwartungserwartungen }\end{array}$ & Institution \\
\hline 5 & Koordinationseffekte & Institution \\
\hline 6 & Komplementaritätseffekte & Institution \\
\hline
\end{tabular}

Quelle: Eigene Darstellung in Anlehnung an Koch, J. (2007), S. 6; Sydow, J. et al. (2005), S. 7.

Bereits aus der Kontextbezeichnung wird deutlich, dass die einzelnen Selbstverstärkungsformen aus unterschiedlichen Forschungsfeldern stammen. Im Folgenden sollen daher die verschiedenen Mechanismen auf ihre Übertragbarkeit in einen organisationalen Kontext hin analysiert werden, um deren Relevanz für die vorliegende Fragestellung überprüfen zu können. Eine Differenzierung von Anbieter- und Konsumentenperspektive erweist sich dabei als hilfreich, da in dieser Arbeit ein klarer Fokus auf dem anbietenden Handlungssystem, der Unternehmung, liegt. Die Kundenperspektive wird zwar nicht außer Acht gelassen, fließt jedoch als die strategischen Entscheidungen des Unternehmens beeinflussender Kontextfaktor nur bedingt mit in die Analyse ein.

Economies of Scales bzw. steigende Skalenerträge liegen auf Anbieterseite vor, sofern bei proportional zunehmendem Faktoreinsatz der Output überproportional steigt. ${ }^{97}$ Dieser Effekt kann auf unterschiedliche Ursachen zurückgeführt werden. Ein wesentlicher Faktor ist die Kostenstruktur der hergestellten Produkte. Je höher die

97 In der Literatur wird mitunter zwischen statischen und dynamischen Skalenerträgen unterschieden. Diese Differenzierung wird hier nicht vorgenommen. Vgl. hierzu ausführlich Junius, K. (1997); Ackermann, R. (2001), S. 59-61. 
anteiligen Fix-kosten an den Gesamtkosten sind, desto stärker sinken die Durchschnittskosten bei Ausweitung der Produktion. Dem ,Gesetz der Massenproduktion'98 folgend, lassen sich so mit steigender Ausbringungsmenge Fixkostendegressionseffekte erzielen. Steigende Skalenerträge ziehen per se allerdings noch keine Selbstverstärkungseffekte nach sich. Eine positive Rückkopplungsschleife bildet sich erst dann heraus, wenn die Increasing Returns die getroffenen (strategischen) Entscheidungen bestätigen und einen weiteren Anstieg der Skalenerträge zur Folge haben. Es entsteht eine „Erfolgsspirale’ aus sich gegenseitig beeinflussenden und verstärkenden Returns und organisationalen Entscheidungen.

Ähnlich wie schon bei den Economies of Scale, lassen sich auch Economies of Scope auf der Anbieterseite verorten. Diese treten dann auf, wenn sich dieselben Inputs für die Herstellung mehrerer Produkte nutzen lassen. In der Literatur werden dazu drei Fälle unterschieden: Die sog. ,Kuppelproduktion' beschreibt den ersten Fall, in dem sich durch die technische Unteilbarkeit der Herstellung zwangsläufig Kostenvorteile durch Verbundvorteile ergeben. Ein zweites Beispiel bilden Portfolioeffekte, indem bei der gleichzeitigen Entwicklung unterschiedlicher Produkte die Flop-Risiken breiter gestreut werden können. Schließlich lassen sich als dritter Verbundvorteil durch die Mehrfachnutzung von Kapazitäten, wie beispielsweise Archivmaterial für verschiedene Redaktionen im Medienbereich, ebenfalls die Durchschnittskosten senken. ${ }^{99}$ Auch hier gilt, dass erst die akteursgeleitete Entscheidung, aufgrund der Verbund- und Synergieeffekte, bestehende Strategien fortzusetzen bzw. zu intensivieren, die selbstverstärkende Schleife zur Generierung weiterer Economies of Scope in Gang setzt.

Netzexternalitäten sind stark technologiegetrieben und konstituieren sich über den Zusammenhang zwischen Verbreitung (der Technologie) und dem unmittelbaren 
Anwendernutzen. Ein prägnantes Beispiel für direkte Netzexternalitäten liegt in der Verbreitung des Telefonnetzes. Je mehr Anwender über ein Telefonnetz miteinander verbunden sind, desto höher steigt der Nutzen jedes einzelnen Anwenders. Indirekte Netzexternalitäten liegen dann vor, wenn - wie im Beispiel von Hard- und Software der Nutzen eines Produkts mit der Kompatibilität komplementärer Waren und Dienstleistungen korreliert. ${ }^{100}$ Aus beiden Beispielen wird deutlich, dass Netzeffekte vornehmlich technologiegetrieben sind und sich auf Nutzen und Anwendung aus Konsumentenperspektive beziehen. Aufgrund des dieser Arbeit zugrunde liegenden organisationalen Fokus sowie der Betrachtung nicht technologischer Güter, finden Netzexternalitäten im weiteren Verlauf nur noch eingeschränkte Berücksichtigung.

Eine weitere Ursache für positive Rückkopplung liegt in Lerneffekten, die sowohl in einem technologischen als auch institutionellen Kontext auftreten können. Der wissenschaftliche Begriff des Lernens blickt auf eine lange Tradition zurück und fußt dabei auf der Konzeption der ,Logik der Veränderung'. ${ }^{101}$ Während der Ausgangspunkt von Lerntheorien auf individueller Ebene zu finden ist, ${ }^{102}$ wurden analog mit dem Terminus ,Organisatorisches Lernen ${ }^{\prime 103}$ Lernprozesse auf einen organisationalen Kontext übertragen. ${ }^{104}$ Gemäß diesem kollektiven Lernverständnis ist Lernen zwar auf individuelles Denken und Handeln zurückführbar, jedoch bildet die Organisation als Wissenssystem den Referenzrahmen, in welchem diese Lernprozesse stattfinden. In diesem Sinne konstituieren Organisationen spezifische Lernmuster - ,collective minds' - als prägende und das kollektive Handeln beeinflussende Schemata. ${ }^{105}$ Gerade in dieser Interdependenz zwischen gelernten Mustern und den daraus resultierenden Ent-

\footnotetext{
100 Vgl. Ackermann, R. (2001), S. 62-64.

101 Vgl. Schreyögg, G. (2006), S. 544.

102 Individuelle Lerntheorien gehen auf behavioristische Ansätze zurück. Wegweisend hierfür war das sog. ,Stimulus-Response Paradigma'. Vgl. Skinner, B.F. (1938).

103 Vgl. hierzu ausführlich March, J.G./Olsen, J.P. (1979), S. $12 f$.

104 Vgl. Schreyögg, G. (2006), S. 550.

105 Vgl. Willke, H. (1996), S. $287 f$.
} 
scheidungen der beteiligten Akteure können sich selbstverstärkende Effekte herausbilden. ${ }^{106}$ Die Entscheidung, ein spezielles Marktsegment zu bearbeiten und gleichzeitig alternative strategische Geschäftsfelder auszublenden, kann beispielsweise zur Ausbildung sehr spezifischer Kompetenzen führen. Diese erhöhen im Umkehrschluss die Attraktivität, das bisherige, ,gelernte' Geschäftsfeld weiter zu bearbeiten. ${ }^{107}$ Neben dieser reinen Organisations- bzw. Angebotsperspektive lassen sich pfadrelevante Lernprozesse im Sinne von Habitualisierungseffekten durch eine Betrachtung des Zusammenspiels mit der Konsumentenseite ergänzen. ${ }^{108}$ Strategische Entscheidungen auf Organisationsebene (z. B. die Einführung von Technologien, aber auch die Etablierung spezifischer Geschäftskonzepte) können zu einer erzieherischen Gewöhnung bzw. Habitualisierung von Kundenverhalten führen. Die Konsumenten prägen auf das jeweilige Angebot abgestimmte, Consumption Skills' aus, welche die weitere Nachfrage nach genau dem bestehenden Angebot verstärken und die Adaption von Alternativen aufgrund nur unzureichend ausgebildeter Fähigkeiten zunehmend unmöglich werden lässt. ${ }^{109}$

Auf kognitiver Ebene, aber auch in ähnlich wechselseitiger Interaktion zwischen Kunden und Anbieter, lassen sich adaptive Erwartungen als weitere Ursache für Rückkopplungsschleifen ausmachen. In der Organisation werden (strategische) Entscheidungen getroffen, weil erwartet wird, dass die Konsumenten gerade diese strategischen Entscheidungen erwarten. Die Kunden wiederum erwarten, dass bestimmte

Vgl. Ackermann, R. (2001), S. 67-72.

107 In engem Zusammenhang steht der Ansatz der Kernkompetenzen, deren überdurchschnittlicher Anfangserfolg in nur wenig experimentierfreudigem, verwandtem Lernen resultiert und eine Verfestigung bestehender organisationaler Handlungsschemata nach sich ziehen kann. Vgl. hierzu Leonad-Barton, D. (1992); Levitt, B./March, J.G. (1988).

108 Wie oben stehend erwähnt, liegt ein klarer Fokus auf der handelnden Organisation, jedoch wird genau dann die Konsumentenseite in die Betrachtung mit einbezogen, wenn ein direkter $\mathrm{Zu}$ sammenhang zwischen strategischen Entscheidungen auf Organisationsebene und Konsumentenhandlung vermutet wird.

109 Vgl. Stigler, G.J./Becker, G.S. (1977), S. 76-90; Wathieu, L. (2004), S. 587-596. 
Entscheidungen auf Organisationsseite verabschiedet werden, so dass sie bereits im Vorfeld ihr Verhalten auf das erwartete Eintreten der (strategischen) Entscheidungen seitens der Organisation einstellen. ${ }^{110}$ In einem wechselseitigen Prozess bestätigen sich die Erwartungen sukzessive selbst.

Wie schon die Selbstverstärkung durch Erwartungserwartungen beziehen sich Koordinationseffekte als weitere Ursache für positive Rückkopplung auf einen institutionellen Kontext, ähneln in ihrer Wirkweise allerdings stark den (technologiegetriebenen) direkten Netzexternalitäten. ${ }^{111}$ Analog zu den direkten Netzexternalitäten steigt im Fall der Koordinationseffekte der Nutzen (sich z. B. an bestimmte Regeln zu halten) durch die steigende Verbreitung (dieser Regeln) insgesamt. Übertragen auf einen Organisationskontext können selbstverstärkende Koordinationseffekte so z. B. bewirken, dass sich innerhalb eines Gesamtsystems die strategische Ausrichtung verschiedener Subsysteme zunehmend angleicht. Je regelkonformer sich die einzelnen Subsysteme bezüglich des Gesamtsystems verhalten, umso leichter gestaltet sich die gegenseitige Interaktion. Im Sinne eines Benchmarkings kann dies zu einer stark vereinheitlichten strategischen Ausgestaltung von miteinander verbundenen Organisationen in einem Gesamtsystem führen. Auch innerhalb eines Subsystems können sich strategische Entscheidungen und - in Anlehnung an kollektive Lernprozesse - Denkschemata stark einander annähern und uniformieren. Ab- und aufeinander eingestimmtes Systemverhalten ist die Folge.

Auch die Wirkweise von Komplementaritätseffekten als letzte Ursache positiver Selbstverstärkung wurde bereits in Teilen schon anhand der vorherigen Rückkopplungsmechanismen erörtert. Während sich durch Economies of Scope Verbundvorteile bei der technischen Herstellung von Gütern einstellen, können Komplementarität-

110 Pierson, P. (2000), S. 254, 258. Es wird in diesem Zusammenhang von einem self-fulfilling character of expectations' gesprochen.

111 Vgl. Ackermann, R. (2001), S. $92 \mathrm{f}$. 
seffekte auf organisationaler Ebene zu Verbundvorteilen innerhalb einer Organisation sowie zwischen Organisation und Kontextfaktoren führen. Dies sei am Beispiel von vertikaler Integration in einem System erläutert: Zur Steigerung der Produktionskapazität werden konzerneigene Zuliefererbetriebe aufgebaut, die sich an den Bedürfnissen des Produktionsbetriebs ausrichten. Die Steigerung des Produktionsvolumens bewirkt einen erhöhten Auftragseingang der Zulieferer, welche wiederum die weitere Produktionssteigerung des Kernunternehmens ermöglichen. So richten sich die einzelnen Systemeinheiten in einem co-evolutorischen Prozess komplementär aneinander aus. Die zirkulär-verstärkende Rückkopplung resultiert in einer zunehmenden Verflechtung der einzelnen Betriebe.

Das breite Spektrum von Selbstverstärkungsmechanismen verdeutlicht neben dem interdisziplinären Ursprung auch die Vielfältigkeit der Bezugsebenen und Analyseperspektiven. In logischer Konsequenz ist es daher empirisch sehr schwer, die einzelnen Rückkopplungstypen vollkommen trennungsscharf voneinander abzugrenzen; vielmehr können sich einzelne Mechanismen überlagern, sequentiell aneinanderreihen, jedoch auch ineinander greifen oder sich kannibalisieren. Gerade in der Komplexität und diffizilen Wirkweise der positiven Rückkopplungen liegt die besondere Herausforderung der pfadtheoretischen Analyse, die im empirischen Teil der vorliegenden Arbeit detailliert diskutiert wird.

\subsubsection{Lock-In und Rigidität}

Historizität und positive Rückkopplungen geben bereits erste Anhaltspunkte auf einen sich herausbildenden (Entscheidungs-)Pfad, von Pfadabhängigkeit lässt sich jedoch erst sprechen, sofern sich der Zustand eines Lock-Ins als Übergang zur dritten Phase pfadabhängiger Prozesse eingestellt hat.

Die soeben beschriebenen Selbstverstärkungskräfte wirken dergestalt auf die organisatorische Entscheidungskultur ein, dass mit fortschreitendem Verlauf der Hand- 
lungsspielraum immer weiter eingeschränkt wird. Dem Druck der Selbstverstärkung folgend, werden und können Entscheidungen nur noch einem festen Muster entsprechend getroffen werden. Sofern eine kritische Masse erreicht wird, genug ,Momentum' generiert ist, befindet sich die Organisation im Zustand eines Lock-Ins. Der Prozess ist irreversibel, d. h. die Kontingenz der Ausgangsbedingungen nicht wiederherstellbar. Mit dem Lock-In liegt organisatorische oder - im Fall strategischer Entscheidungen - strategische, pfadbedingte Rigidität vor, die sich in der trägen Gleichförmigkeit des Entscheidungsverlaufs äußert.

Sofern selbst Wandlungsbemühungen scheitern oder Marktbewegungen keine Änderung hervorrufen, ist das pfadabhängige System immun gegenüber Störgrößen und intentionaler Einflussnahme.

Ursächlich hierfür sind drei zu unterscheidende Fälle von Ignoranz: ${ }^{112}$ Im ersten Fall kennt die betroffene Organisation keine alternativen Entscheidungswege, da z. B. die jeweilige Ressourcen- oder Kompetenzausstattung keine divergierende Unternehmensstrategie zulässt (,Nicht wissen'). Der Pfad kann nicht verlassen werden. Im zweiten Fall resultiert die Änderungs-Ignoranz aus der mangelnden Wahrnehmung von Handlungsalternativen (,Nicht beachten'). Der Pfad wird aus Gründen limitierter Einsicht und Blindheit nicht verlassen. Schließlich beschreibt der dritte Fall von Ignoranz (,Nicht wissen wollen') die bewusste Entscheidung einer Organisation, keine alternativen Handlungswege zu beschreiten. Der Pfad soll nicht verlassen werden. ${ }^{113}$ Der letzte Fall liegt z. B. bei einem ,ökonomisch effizienten Pfad' vor, bei dem volles Bewusstsein über das bestehende Abhängigkeitsverhältnis vorhanden ist, die Organisati-

112 Ignoranz zeichnet sich dadurch aus, dass jemand etwas nicht weiß, nicht wissen will oder nicht beachtet. Vgl. Duden (1989), S. 752.

113 Vgl. hierzu die Unterscheidung von ,unable', ,unaware' und ,unwilling' von Régner, P. (2005) sowie Stufe 5 der Analyseschritte strategischer Pfade von Koch (2007a), S. 287. 
on sich jedoch nicht wandeln möchte. ${ }^{114}$ In Zeiten wirtschaftlichen Erfolgs und stabiler Wettbewerbsverhältnisse ist dies sicherlich als problemlos einzustufen. Sofern sich die Marktbedingungen wandeln, können sich pfadbedingte Verfestigungen jedoch schnell als entscheidende Hemmnisse für die Organisation herausstellen. ${ }^{15}$ In diesem Sinne sind pfadabhängige Prozesse generell als problematisch zu beurteilen, deutet doch allein der Begriff ,Abhängigkeit' auf eine eingeschränkte Entscheidungsfreiheit und damit auf die Schwierigkeit, wenn nicht sogar Unmöglichkeit, intentionaler Gegensteuerungsmaßnahmen hin.

Selbst wirtschaftlich effiziente Unternehmensstrategien können zwar kurzfristig effektiv sein und das angestrebte (Gewinn-)Ziel erreichen, sich jedoch langfristig als Sackgasse erweisen, da nicht nur die Zielerreichung nicht mehr gewährleistet, sondern zusätzlich ineffizient gewirtschaftet wird. Aufgrund der pfadabhängigen Kräfte, die den (strategischen) Handlungsspielraum nachhaltig limitieren, ist es kaum mehr möglich, die Rigidität des einst effizienten Pfades aufzubrechen.

Im Zustand des Lock-Ins ist die Entscheidungsfreiheit bereits so stark eingeschränkt, dass selbstverstärkende Kräfte nicht mehr aktiv wirken müssen, um die Rigidität aufrechtzuerhalten; dennoch können diese den Entscheidungsprozess weiterhin verstärkend oder stabilisierend beeinflussen.

Zusätzlich ist die Existenz weiterer - nicht verstärkender, sondern einseitig linear wirkender - Stabilisationsfaktoren möglich, welche zwar nicht ursächlich für die Ausbildung der Pfadabhängigkeit sind, jedoch als Ergänzung stabilisierend auf den gleichförmigen Strategieverlauf einwirken.

114 Einen ,effizienten' Pfadverlauf zu erkennen stellt den Beobachter dabei vor ganz besondere Herausforderungen, da für die jeweilige Organisation ja zunächst keine Veranlassung zum Wandel besteht. Dennoch ist von einem Pfad zu sprechen, sofern die Bedingungen der Historizität, positiven Rückkopplung und des Lock-Ins gegeben sind.

115 Siehe hierzu die Diskussion zu Kernkompetenzen, die zu Kernrigiditäten werden können. Vgl. Leonard-Barton, D. (1992), S. 112-126. 
Die nachfolgende Abbildung 7 verdeutlicht den beschriebenen Pfadentstehungsverlauf. Von einer tatsächlichen Pfadabhängigkeit lässt sich erst sprechen, sofern auch ein Lock-In in Phase III erreicht wird. Erst dann ist abweichendes Verhalten nahezu unmöglich. Durch die Einteilung in drei Phasen wird der dynamische Charakter der Pfadtheorie besonders klar.

Abbildung 7: Drei-Phasen-Modell der Pfadentstehung

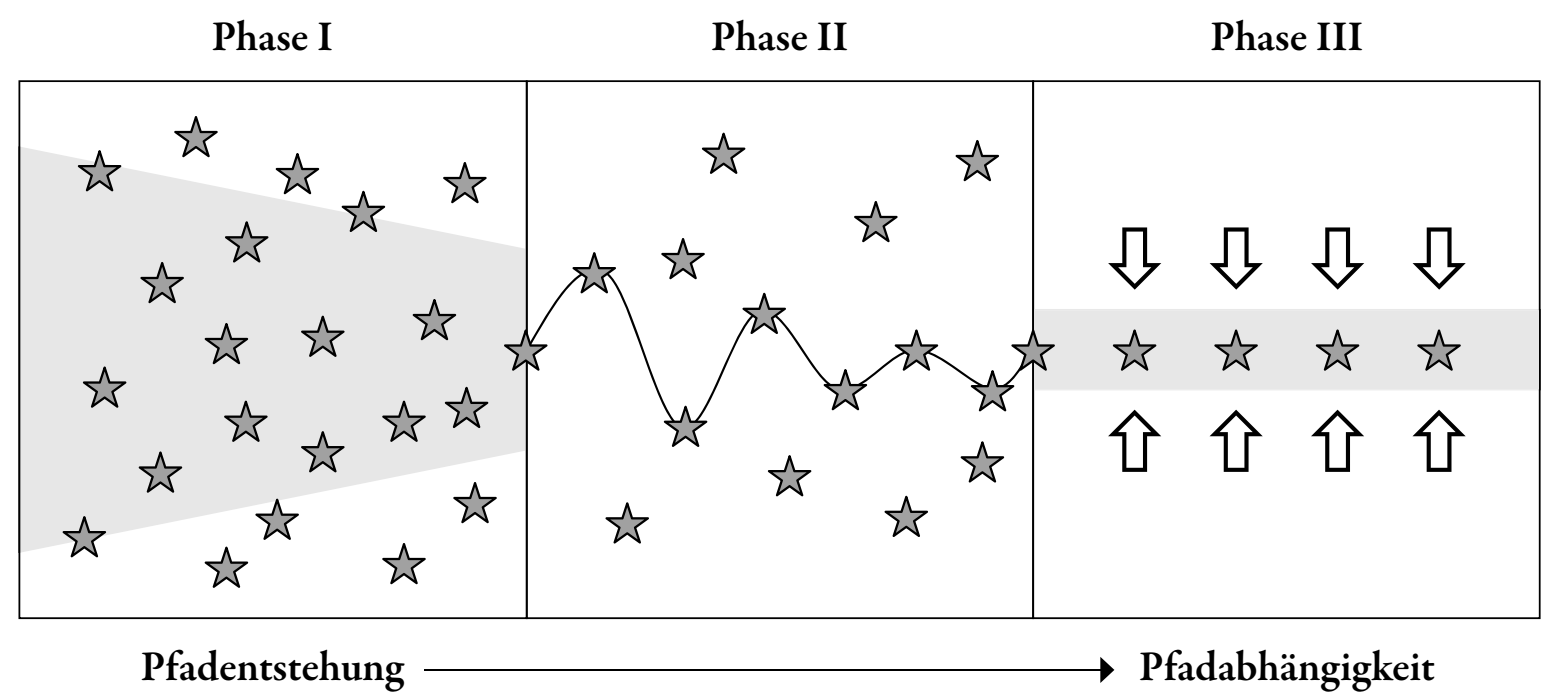

Quelle: Eigene Darstellung in Anlehnung an Schreyögg, G. et al. (2003), S. 272.

Der graue Schatten in Phase I unterstreicht die historische Vorprägung von Organisationen unter ansonsten kontingenten Anfangsbedingungen. Der Übergang von Phase I zu Phase II ist durch das ,Critical Juncture' gekennzeichnet. Ereignisse und Entscheidungen mit ungewissem Ausgang (,Small Events') werden zum Auslöser von Selbstverstärkungsprozessen, so dass Small Events in der rückblickenden Beobachtung als kritische Meilensteine und eben nicht als unbedeutende Ereignisse einzustufen sind. Der Übergang von Phase II zu Phase III ist schließlich derjenige kritische Zeitpunkt, der den Beginn des Lock-Ins und somit der prozessualen Irreversibilität beschreibt. Die einseitig gerichteten Pfeile in Phase III stellen eine Erweiterung des originären 
Drei-Phasen-Modells von SCHREYÖGG ET AL. dar, indem sie das Element der stabilisierenden Faktoren in den Pfadprozess integrieren.

\subsubsection{Zwischenfazit zur Theorie der Pfadabhängigkeit}

In einem ersten Schritt wurden die zentralen Beiträge zur ökonomisch orientierten Pfadabhängigkeitstheorie erläutert sowie ein weiter und enger Pfadbegriff eingeführt. Ausgehend von einem engen Pfadkonzept wurden in einem zweiten Schritt die zentralen Phasen der Historizität, Selbstverstärkung und des Lock-Ins in einem organisationalen Kontext ausführlich diskutiert. Die klassischen Pfadbedingungen wurden dafür um einen anfänglich eingeschränkten Kontingenzbegriff und die Aufhebung des vollständigen Determinismus in der Phase des Lock-Ins erweitert.

Der von der Pfadtheorie konstatierte eingeschränkte Handlungsspielraum sowie der Zustand eines pfadbedingten Lock-Ins in Phase III beschreiben Phänomene von organisationaler Rigidität und Trägheit. Zu diesen besteht in der Literatur bereits ein breiter Kanon an Konzepten und Theorien. Zwar kann im Rahmen dieser Arbeit kein vollständiges Bild der Theoriediskussion abgegeben werden; dennoch sollen im folgenden Kapitel einige zentrale Ansätze zu organisationaler Trägheit vorgestellt und mit der Pfadtheorie in Verbindung gebracht werden.

Ziel des synoptischen Vergleichs ist zum einen die Generierung alternativer Erklärungsansätze für empirische, nicht pfadbedingte Trägheitsphänomene. Zum anderen hilft die Gegenüberstellung divergierender Theoriegebilde, den Blick auf die Pfadtheorie selbst zu schärfen und somit das theoretische Grundverständnis in Vorbereitung auf die empirische Pfadanalyse in Kapitel 4 zu vertiefen. 


\subsection{Theoriediskussion zu organisationaler Trägheit}

Im allgemeinen Sprachgebrauch ist unter ,Rigidität' das „starre[...] Festhalten an früheren Einstellungen, Gewohnheiten o. ä. " ${ }^{116}$ zu verstehen. Analog hierzu hat sich der ursprünglich aus der Physik stammende Begriff ,Trägheit' ${ }^{117}$ in der Strategie- und Organisationsforschung etabliert: „Absent to other forces, inertia describes the tendency to remain with the status quo and the resistence to strategic renewal outside the frame of current strategy. " 118 Trägheit oder Rigidität implizieren somit organisationale Beharrungstendenzen gegenüber (strategischem) Wandel. Eine Organisation hält an etablierten strategischen Mustern fest.

Zur Ursachenklärung von organisationaler Trägheit sind in der wissenschaftlichen Forschung eine Vielzahl von Konzepten und Theorien entwickelt worden. Wesentliche Ansätze sollen nun in ihren Grundzügen dargelegt und diskutiert werden. ${ }^{119} \mathrm{Da}$ die vorliegende Arbeit das Ziel verfolgt, eine Pfadanalyse an einem konkreten Fallbeispiel vorzunehmen, kann und soll an dieser Stelle keine lückenlose Theoriediskussion zu organisationaler Trägheit stattfinden. Vielmehr gilt es, die Kernargumente der zentralen Ansätze darzustellen, einem inhaltlichen Vergleich mit der Pfadtheorie zu unterziehen, voneinander abzugrenzen bzw. zu integrieren. Diese konzeptionelle $\mathrm{Zu}$ sammenführung wird in Kapitel 2.4.5 vorgenommen.

116 Vgl. Duden (1989), S. 1257.

117 Trägheit beschreibt die „Eigenschaft jeder Masse, ihren Bewegungszustand beizubehalten, solange keine äußere Kraft einwirkt, die diesen Zustand ändert.“ Duden (1989), S. 1545.

118 Huff, J.O. et al. (1992), S. 56.

119 Die Literaturübersicht beschränkt sich auf einige zentrale Erklärungsansätze. Alle Ansätze nähern sich - teilweise vor unterschiedlichem erkenntnistheoretischem Hintergrund - dem Phänomen der Trägheit in Organisationen. Aufgrund des gemeinsamen Erklärungsinteresses sind die einzelnen Ansätze nicht immer vollkommen trennungsscharf voneinander abzugrenzen. Daher wurde versucht, die jeweils zentralen Argumentationsstränge zur Erklärung von organisationaler Trägheit herauszustellen. 


\subsubsection{Imprinting}

Der Imprinting-Ansatz betrachtet Organisationen aus einer biographischen Perspektive, weist auf den starken Einfluss früher Entscheidungen auf den weiteren Unternehmensverlauf hin und betont somit dessen historische Verankerung. ${ }^{120}$ Unabhängig davon, ob externe oder interne Faktoren die frühen Entscheidungen herbeigeführt oder geprägt haben, können diese auf nachfolgende Entscheidungen ausstrahlen. Spätere Systemzustände lassen sich zwar vor dem Hintergrund anfänglicher Entscheidungen analysieren, jedoch nicht im gesamten Prozessverlauf rekonstruieren. ${ }^{121}$ Hierbei wird vor allem auf die Gründungsphase eines Unternehmens abgestellt, in welcher grundlegende Entscheidungen zu Eigentümer- und Governance-Strukturen, Marktbearbeitung, Personalpolitik und Organisationsstrukturen getroffen werden. ${ }^{122}$ Neben dem starken Einfluss von Gründungsentscheidungen können auch Ereignisse und Entscheidungen zu einem späteren Zeitpunkt den Organisationsverlauf entscheidend prägen. ${ }^{123}$ Wichtig ist, dass diese kritischen Ereignisse auf die weitere Unternehmensentwicklung ausstrahlen und die Veränderungsvarianz einschränken.

Der Imprinting-Ansatz weist somit starke Analogien zu der in Phase I des DreiPhasen-Modells der Pfadentstehung beschriebenen Historizität (,history matters') von Ereignisketten und Entscheidungsverläufen auf. Wie und unter welchen Bedingungen die einzelnen Anschlussentscheidungen zusammenhängen und miteinander verknüpft sind (linear, zirkulär-rekursiv, zirkulär-verstärkend), ist allerdings nicht Gegenstand des Imprinting-Ansatzes.

120 Vgl. Stinchcombe, A.L. (1965), S. 142-193; Boeker, W. (1989), S. 489-515.

121 Vgl. Burton, M.D./Beckman, C.M. (2005); Kimberly (1987), S. 223-237.

122 KIMBERLY differenziert vier Klassen von Grundsatzentscheidungen: Governance decisions, domain decisions, expertise decisions und design decisions. Vgl. Kimberly, J.R. (1987), S. $234 \mathrm{f}$.

123 "Imprinting refers to a process by which events occurring at certain key developmental stages have persisting, if not lifelong, consequences." Hannan, M.T. et al. (1996), S. 507. 
Eine ergänzende Betrachtung stellt auf die sog. ,Role Imprints' ab. Unternehmensgründer und frühe Organisationsmitglieder erschaffen bzw. prägen die Rollenerwartungen für das jeweilige Handlungssystem - und somit auch an die Handlungen neu gewonnener Mitglieder. Neben den normativen Umwelterwartungen beeinflussen diese internen Rollenfestlegungen das zukünftige Mitarbeiterverhalten entscheidend, so dass die Handlungsvarianz der Unternehmensteilnehmer bereits durch die Historie richtungsweisend beeinflusst wird. Ein Wandel und das Aufbrechen etablierter Strukturen werden aufgrund von formalisierten Rollenerwartungen erheblich erschwert. ${ }^{124}$

Unter Vorgriff auf die konzeptionelle Zusammenführung in Kapitel 2.4.5 lässt sich bereits erkennen, dass die Historizität von Entscheidungsprozessen des Imprinting-Ansatzes nicht mit den zentralen Annahmen der Pfadtheorie konkurriert, sondern mit der historischen Vorprägung von pfadabhängigen Prozessen in Einklang zu bringen ist.

\subsubsection{Structural Inertia}

Im Gegensatz zum Ansatz des Imprintings führt die evolutionstheoretisch motivierte Theorie der ,Structural Inertia' ${ }^{125}$ Widerstand gegenüber Wandel auf die Formalisierung und Standardisierung von Zielen und Handlungsmustern in Organisationen zurück. Zur Zielerreichung, einen größtmöglichen Fit zwischen den Ressourcen der Organisation und der Umwelt herzustellen, erprobt eine Organisation unterschiedliche Variationsmöglichkeiten. Ist eine erfolgreiche Variation von Unternehmensressourcen gefunden worden, gilt es, diese dauerhaft zu sichern und vor weiteren Veränderungen zu bewahren.

HANNAN/FREEMAN (1984) beziehen sich konkret auf die Größen von Verlässlichkeit (Reliability) und Zurechnungsfähigkeit (Accountability), welche den Unterneh-

\footnotetext{
124 Vgl. Burton, M.D./Beckman, C.M. (2005).

125 Vgl. Hannan, M.T./Freeman, J. (1984), S. 149-164.
} 
men dabei helfen, Stabilität aufzubauen und sich so im Markt zu behaupten. Diese Formalisierung von Organisationen geht mit der Ausbildung stabiler Strukturen und Routinen einher, wodurch auf der einen Seite Transparenz, Vorhersagbarkeit und Zuverlässigkeit gefördert, auf der anderen Seite jedoch Wandel und Flexibilität limitiert wird. ${ }^{126}$ Die Tendenz zu struktureller Trägheit ist umso stärker, je älter und größer Organisationen sind. ${ }^{127}$ Die Korrelation zwischen dem Alter einer Organisation und ihrer Trägheit erklärt sich durch die im Zeitverlauf zunehmende Formalisierung und Standardisierung ihrer Routinen. Ähnlich wird der Zusammenhang zwischen Größe und Trägheit hergeleitet: Je größer Organisationen werden, desto höher ist auch ihr Komplexitätsgrad und die Gefahr des Kontrollverlusts. Zur Gegensteuerung werden formalisierte Strukturen und Routinen ausgebildet. ${ }^{128}$

Konkret benennen HANNAN/FREEMAN sieben organisationsinterne sowie -externe Faktoren als Ursache für Structural Inertia: Sunk Costs, Kommunikationsstrukturen, interne Wertvorstellungen sowie institutionelle Normen bilden interne Komponenten, wohingegen gesetzliche Markteintritts- und -austrittsschranken, unvollständige Informationen sowie Legitimationsbarrieren als externe Quellen organisationaler Rigidität angesehen werden. ${ }^{129}$

Sunk Costs entstehen beispielsweise durch die Mobilisierung von Ressourcen in Form von irreversiblen Investitionen in Zeit oder persönlichen Einsatz, deren Höhe ausschlaggebend für den Widerstand gegenüber Wandel ist. Hohe, und vor allem unwiederbringliche Investitionen in bestimmte Ressourcen erhöhen somit die Wahrscheinlichkeit von Trägheit. ${ }^{130}$

126 "Thus, the very characteristics that give an organization stability also generate resistance to change.” Kelly, D./Amburgey, T.L. (1991), S. 593.

127 Vgl. Betton, J./Dess, G.G. (1985), S. 752.

128 Vgl. Kelly, D./Amburgey, T.L. (1991), S. 594.

129 Vgl. Hannan, M.T./Freeman, J. (1989), S. 66-90; Gresov, C. et al. (1993), S. 181-208.

130 Vgl. Schreyögg, G. (2006), S. 502. 
Als weiteres Beispiel können unvollständige Informationen dazu führen, dass Umweltveränderungen und somit die Notwendigkeit zum Wandel in der Organisation erst gar nicht wahrgenommen werden. Die Ursache der unvollständigen Informationen ist in den informellen Strukturen zu suchen, deren Informationskanäle über die Aufnahme und Weiterleitung von Informationen in der Organisation bestimmen. ${ }^{31}$

Die Theorie der Structural Inertia beschreibt zusammenfassend ein breites Spektrum von Ursachen, anhand derer sich organisationale Beharrungstendenzen sowie Widerstand gegenüber Wandel erklären lassen. ${ }^{32}$ Wie schon der Imprinting-Ansatz schließt auch das Structural Inertia-Konzept die Dimension ,Zeit' in seinen Erklärungsansatz mit ein und betont die Nachwirkungen von frühen Entscheidungen (z. B. unwiederbringliche Investitionen im Sinne von Sunk Costs) auf spätere Ereignisse. Im Gegensatz zu der rein historischen Betrachtung des Imprinting liefert der Structural Inertia-Ansatz darüber hinaus konkrete (strukturelle) Ursachen für Trägheit und benennt damit klare Kausalitäten. Gemeinsamkeiten und Unterschiede zur Theorie der Pfadabhängigkeit werden in Kapitel 2.4.5 ausführlich erläutert wird.

\subsubsection{Lerntrajektorien und Kernrigiditäten}

Ein weiterer Ansatz zur Analyse von Trägheiten und Rigiditäten in Organisationen basiert auf der Tradition des Ressource-Based-Views und beschäftigt sich mit unternehmensspezifischen Kompetenzen oder Capabilities. ${ }^{133}$

Zum Aufbau eines strategischen Wettbewerbsvorteils müssen Unternehmen neben den ,allgemeinen' Produktionsressourcen wie Personal und Kapital ,spezifische' Un-

131 Vgl. Hannan, M.T./Freeman, J. (1989), S. 67.

132 Für eine detaillierte Darstellung des Ansatzes siehe Hannan, M.T./Freeman, J. (1989), S. 66-90.

133 Eng verwandt mit den Begriffen ,Kernkompetenz', ,Fähigkeit', ,Routine’ oder ,Best Practice’ leisten SCHREYÖGG/KLIESCH-EBERL eine konzeptionelle Übersicht und filtern drei zentrale Eigenschaften von Capabilities heraus: Problem-solving und Complexity, Practicing und Success sowie Reliability und Time. Vgl. Schreyögg, G./Kliesch-Eberl, M. (2007), S. 913-933. 
ternehmensressourcen wie Kompetenzen, Fähigkeiten oder Wissen entwickeln. Diese sollten möglichst wertig und selten sowie schwer von Wettbewerbern zu imitieren und zu substituieren sein (VRIN-Kriterien). ${ }^{134}$

Eine Erweiterung stellt das Konzept der Kernkompetenzen dar: Unterschiedliche, Erfolg versprechende Unternehmensressourcen werden zu einem „pattern of internal coordination and learning “135 im Sinne einer „spezifischen replizierbaren ,Problemlösungs-Architektur'“136 zusammengefasst, welche ein Unternehmen dazu befähigt, Wettbewerbsvorteile in verschiedenen Geschäftsfeldern aufzubauen. Hierfür ist Beständigkeit notwendig. Kernkompetenzen entstehen durch kollektive Lernprozesse, in welchen spezifische Muster der Auswahl und Verknüpfung von Ressourcen - ähnlich einer Routine - zu Kernkompetenzen verdichtet werden. Deutlich wird hierbei vor allem der historische Charakter von Kernkompetenzen, der auf der einen Seite Einzigartigkeit und Unimitierbarkeit sicherstellt, auf der anderen Seite jedoch zu Trägheit und Rigidität führen kann. ${ }^{137}$

Die Etablierung besonders erfolgreicher Kernkompetenzen, deren tiefe organisatorische Verankerung und das eingespielte Problemlösungsverhalten bergen die Gefahr, nur unzureichend auf Umweltbewegungen reagieren zu können. Sofern die Dynamik der Umwelt, z. B. durch den plötzlichen Wegbruch eines Marktsegments, Neuerungen erfordert, werden die historisch gewachsenen ,Kernkompetenzen’ zu ,Kernrigiditäten', da die Organisation nicht in der Lage ist, sich den geänderten Marktverhältnissen anzupassen. ${ }^{138}$

134 Vgl. Steinmann, H./Schreyögg, G. (2005), S. 260.

135 Pralahad, C.K./Hamel, G. (1990), S. 84.

136 Steinmann, H./Schreyögg, G. (2005), S. 261.

137 Vgl. Leonard-Barton, D. (1992), S. 118-121.

138 Vgl. Leonhard-Barton, D. (1992), S. 111-125; Dougherty, D. (1995), S. 113-135; siehe hierzu auch den Begriff der ,competency trap': Levitt, B./March, J.G. (1988), S. $322 f$. 
Verdeutlicht wird dieser Zusammenhang durch die Form des ,ExploitationLernens': ${ }^{139}$ Im Gegensatz zu ,Explorations-Prozessen', ${ }^{140}$ in denen losgelöst von bestehenden Kompetenzen z. B. neue Märkte erobert oder Produkte innoviert werden, bauen exploitative Lernprozesse auf bestehendem Wissen auf und entwickeln es von dort aus weiter ${ }^{141}$ Als Konsequenz verhindert Exploitation-Lernen ein experimentelles Vorstoßen in noch unbekannte Bereiche zugunsten der Replikation und Verbesserung bestehender Kompetenzen. ${ }^{142}$ Es bilden sich Lerntrajektorien heraus, welche auf bereits bekannten Kompetenzen basieren, so dass sich etablierte (strategische) Muster verfestigen. Neues, nicht verwandtes Lernen wird verlernt.

Ein ähnliches Phänomen beschreibt das bereits erörterte ,Ikarus Paradox’. Besonders erfolgreiche Unternehmen laufen Gefahr, ihre Arbeitsabläufe sehr stark zu fokussieren und ihre Kompetenzausstattung zuzuspitzen. ${ }^{143}$ Der Markterfolg einer bestimmten Technologie kann so z. B. zu einer Desensibilisierung gegenüber Marktentwicklungen (Substitutionsprodukte, Wettbewerber) führen, so dass gerade die organisatorische Ausrichtung auf den einstigen Erfolgstreiber den ökonomischen Niedergang verursachen kann.

Zusammenfassend lässt sich festhalten, dass der Kompetenz-Ansatz organisationale Rigidität und Trägheit auf Basis von historisch gewachsenen Lern- und Verfestigungstrajektorien erklärt, deren zunächst erfolgreiche Muster Flexibilität und schnelle Reaktionen auf Veränderungen der Rahmenbedingungen erschweren. Trotz des un-

139 "Exloitation includes such things as refinement, choice, production, efficiency, selection, implementation, execution.” March, J.G. (1991), S. 71.

140 "Exploration includes things captured by terms such as search, variation, risk taking, experimentation, play, flexibility, discovery, innovation.” March, J.G. (1991), S. 71.

141 DANNEELS stellt Lerntrajektorien am Beispiel von Produktentwicklungen dar: Produkte entstehen auf Basis bereits bestehender (Kunden- bzw. Technologie-)Kompetenzen, so dass Neuprodukte nicht losgelöst von der etablierten Wissensbasis entwickelt werden. Vgl. Danneels, E. (2002), S. 1095-1121.

142 Vgl. March, J.G. (1991), S. 71-87; Teece, D.J. (1988), S. 256-281.

143 Vgl. Miller, D. (1993), S. 116-138; Miller, D. (1994), S. 325-358. 
terschiedlichen theoretischen Hintergrundes zeigt die Betrachtung von (Kern-) Kompetenzen wie bereits die Theorie der Structural Inertia einen Erklärungsansatz von organisationaler Trägheit auf, der in einer zeitlichen Dimension auf historische Prozesse und in einer kausalen Dimension auf die Reaktivität von strategischen Entscheidungen (der Erfolg von spezifischen Kompetenzen führt zum weiteren Ausbau derselben) Bezug nimmt. Wie bereits angekündigt, erfolgt eine genauere Erörterung von Analogien bzw. Gegensätzen zur Theorie der Pfadabhängigkeit in Kapitel 2.4.5. Zunächst sollen jedoch noch weitere zentrale Ansätze zur Erklärung von organisationaler Trägheit vorgestellt werden.

\subsubsection{Kognitive Verfestigungen}

Kognitive Verfestigungen entstehen auf einer sozial-psychologischen Ebene von bzw. zwischen Individuen und sollen aufgrund der Betrachtung von strategischen Entscheidungen auf Organisationsebene in der vorliegenden Arbeit nur ergänzend diskutiert werden. ${ }^{144}$

Kognitive Verfestigungen können auf vielfältige Arten entstehen: Unter den bereits erörterten Annahmen von begrenzter Rationalität und Selektionsnotwendigkeit ${ }^{145}$ besteht z. B. die Gefahr, akteursbasierte Entscheidungen auf Basis von selektiver Wahrnehmung ${ }^{146}$ zu treffen. Relevante Umweltbewegungen werden nicht erkannt, so dass ein gleichförmiges, träges Entscheidungsverhalten resultiert, ohne die Notwendigkeit zum strategischen Wandel wahrzunehmen.

Ähnlich wirken mentale Modelle (,Mental Models') oder ,Cognitive Maps', ${ }^{147}$ die den Akteuren aufgrund von hoher Umweltkomplexität einen strukturierten und zugleich stark reduktionistischen Orientierungsrahmen bieten. Mentale Modelle sind

144 Vgl. Garud, R./Rappa, M. (1994), S. 344-362.

145 Siehe Kapitel 2.1.3 und 2.1.6 dieser Arbeit.

146 Vgl. Dearborn, W.C./Simon, H.A. (1958), S. 140-144; Walsh, J.P. (1988), S. 873-896.

147 Vgl. Weick, K.E./Bougon, M. (1986), S. 102-135. 
nicht in der Lage, die Gesamtheit potentiell relevanter Informationen abzubilden, erfüllen sie ja gerade den Zweck, die Informationsvielfalt handhabbar und überschaubar verarbeiten zu können. Selektion und vereinfachte Abbilder der Umwelt können somit zu verzerrter Wahrnehmung und einseitigen Perspektiven führen - mit der Gefahr, die Dynamik des relevanten Markts nicht vollständig erfassen zu können. Die Trägheit (strategischer) Entscheidungsprozesse lässt sich somit auf die mangelnde Wahrnehmungsfähigkeit durch verfestigte kognitive Strukturen zurückführen.

Auch der psychologisch motivierte Ansatz des ,Escalating Commitment ${ }^{148}$ basiert auf der subjektiven Wahrnehmung, an einer getroffenen Entscheidung festzuhalten. Im Sinne von psychologischen versunkenen Kosten zieht die mangelnde Einsicht, vergangene Entscheidungen zu revidieren, die Replizierung des konservativen Entscheidungsmusters nach sich.

Formen von individueller Entscheidungsbindung finden sich auch auf kollektiver und organisationaler Ebene wieder. Phänomene wie ,Groupthink ${ }^{149}{ }^{149}$ oder die Herausbildung einer ,Dominant Logic'150 führen zu einer einheitlichen Systemwahrnehmung, indem sich Sichtweisen und Handlungen durch Kräfte wie Kohäsion oder die Vermeidung von kognitiven Dissonanzen einander annähern und vereinheitlichen. Eine Organisation entscheidet als Folge aus einer stark selektiven, systemspezifischen Perspektive, welche einseitiges und rigides Entscheidungsverhalten zur Folge haben kann.

Abschließend lässt sich festhalten, dass die Untersuchung von organisationaler Trägheit aus kognitiv-psychologischer Sicht eine Vielzahl von Erklärungsansätzen bietet. Zentraler Fokus sind die Kognitionen von Akteuren, auf deren Basis zu Trägheit führende Entscheidungen getroffen werden. Obwohl die kognitiv motivierten Ansätze somit im Gegensatz zu Imprinting, Structural Inertia oder dem Kompetenz-Ansatz or-

\footnotetext{
148 Vgl. Staw, B.M. (1976), S. 27-44; Staw, B.M. (1981), S. 577-587.

149 Vgl. ausführlich Janis, I.L. (1982).

150 Vgl. Pralahad, C.K./Bettis, R.A. (1986), S. 485-501.
} 
ganisationale Trägheit aus einer akteursgerichteten Perspektive betrachten, lassen sich Gemeinsamkeiten bezüglich der Analysedimensionen ,Zeit' und ,Kausalität' feststellen. Mentale Modelle oder dominante Logiken ziehen ihre Erklärungskraft wie die anderen Ansätze auch aus einer zeitlichen Dimension, indem sich Strukturen erst im Zeitverlauf verfestigen. Aus der Ursache (eingeschränkte Wahrnehmung) leitet sich schließlich durch kausale Verknüpfung die Wirkung (Stabilität der Entscheidungen) ab.

\subsubsection{Konzeptionelle Zusammenführung}

Zusammenfassend lässt sich festhalten, dass Rigiditäten und Trägheit in Organisationen durch ein breites Spektrum von Forschungsansätzen und Theorien erklärt werden. Die Tatsache, dass der Ursprung der einzelnen Konzepte auf unterschiedliche wissenschaftliche Disziplinen und erkenntnistheoretische Grundlagen zurückzuführen ist und die Betrachtung von Trägheit auf verschiedenen Ebenen abläuft, erschwert eine klare inhaltliche Vergleichbarkeit der unterschiedlichen Ansätze. Dennoch sollen die einzelnen Konzepte zusammengeführt werden, jedoch nicht mit dem Ziel eines detaillierten inhaltlichen Vergleichs oder einer normativen Beurteilung. Vielmehr soll durch die theoretische Übersicht der Blick für das konzeptionelle Fundament und die inhaltliche Ausgestaltung der Pfadtheorie geschärft werden. Angesichts der Fülle von unterschiedlichen Erklärungsansätzen zu organisationaler Trägheit ist dies vor allem dazu notwendig, um den Mehrwert der Theorie der Pfadabhängigkeit zur Erklärung von Rigiditätsphänomenen herauszustellen.

In einem ersten Schritt werden hierfür die einzelnen Forschungsansätze zu organisationaler Trägheit auf konzeptioneller Ebene miteinander in Verbindung gebracht. Wie in Tabelle 3 dargestellt, wurde zu diesem Zweck ein dreigliedriges Kategoriensystem entwickelt, dessen einzelne Stufen sich aus den jeweiligen Dimensionen, auf deren Basis die Theorien argumentieren, zusammensetzen: 
Zeit im Sinne von organisationaler Historizität, Kausalität als UrsacheWirkungsbeziehung innerhalb der Prozesskette sowie die Dimension des Mechanismus, der den Automatismus der (non)linearen Zusammenhänge zwischen Anfangsund Endzustand beschreibt.

Tabelle 3: Vergleichende Einordnung der Theorien zu organisationaler Trägheit

\begin{tabular}{l|l|l|l|l}
\multicolumn{1}{c|}{$\begin{array}{l}\text { Dimension } \\
\text { abstrakt }\end{array}$} & konkret & Imprinting & $\begin{array}{c}\text { Structural inertia } \\
\text { Lerntrajektorien } \\
\text { Kognitive } \\
\text { Verfestigungen }\end{array}$ & $\begin{array}{c}\text { Pfadabhängig- } \\
\text { keit }\end{array}$ \\
\hline Zeit & Historizität & & & \\
\hline Kausalität & Reaktivität & & & \\
\hline Mechanismus & (Non-)Linearität & & & \\
\hline
\end{tabular}

Quelle: Eigene Darstellung.

Die Übersicht lässt erkennen, dass sämtliche vorgestellten Ansätze eine zeitliche Dimension mit einschließen, indem eine retrospektive Prozessperspektive zur Erklärung von organisationaler Rigidität berücksichtigt wird. Dies liegt allein schon in der Natur des zu messenden Trägheits-Konstrukts, da sich Trägheit und Rigidität gerade über den Wandel bzw. Nicht-Wandel im Zeitverlauf definieren. ${ }^{151}$

Bezüglich der zweiten Dimension - Kausalität bzw. Reaktivität - muss eine differenziertere Betrachtung vorgenommen werden.

Während der Ansatz des Imprintings keine Aussagen über die logische Verknüpfung innerhalb der (Entscheidungs)-Prozesskette trifft, benennen die Theorien zu Structural Inertia, Lerntrajektorien, kognitiven Verfestigungen und Pfadabhängigkeiten klare Faktoren und Ursache-Wirkungszusammenhänge zur Entstehung von orga-

151 Siehe hierzu die Definition von ,Trägheit' in Kapitel 2.4 dieser Arbeit. 
nisationaler Rigidität. ${ }^{152}$ Die Gemeinsamkeit dieser Ansätze liegt somit zunächst auf der Beschreibung von reaktiven, historischen Ereignisketten, ${ }^{153}$ deren Ergebnis in Rigidität und Trägheit mündet. Mit Ausnahme der Pfadtheorie wird jedoch offen gelassen, in welcher Form die einzelnen Prozessschritte miteinander verbunden sind.

Diese Lücke schließt die Pfadtheorie mit Hilfe der dritten Analysedimension dem Mechanismus. Besonders deutlich wird dabei der Charakter der Pfadtheorie als Prozesstheorie, indem nicht nur erforscht wird, dass eine historisch fundierte, kausal verknüpfte Ereignis- bzw. Entscheidungskette vorliegt, sondern auch wie die einzelnen Glieder miteinander verknüpft sind. Die Pfadtheorie erklärt somit Trägheit aus dem Prozess selbst heraus. Tabelle 3 ist somit integrativ zu verstehen: Die Theorie der Pfadabhängigkeit vereint die Analysedimensionen der Historizität, der Kausalität und des Mechanismus.

Im Anschluss an diese konzeptionelle Erfassung der Pfadtheorie stellt sich in einem zweiten Schritt die Frage nach der inhaltlichen Ausgestaltung von pfadabhängigen Prozessen im Vergleich zu alternativen Trägheitsphänomenen. Welcher Argumente bedient sich die Pfadtheorie im Gegensatz zu den anderen vorgestellten Theorien zu organisationaler Trägheit? Wie dargestellt, beschreibt auch der Zustand des Lock-Ins organisationale Trägheit als Resultat pfadabhängiger Prozesse. ${ }^{154}$ Der Typus pfadbedingter Trägheit grenzt sich jedoch durch den selbstverstärkenden Prozesscharakter von den traditionellen Trägheitskonzepten ab. Dabei stehen Ursachen und Faktoren, welche gemäß den Ansätzen zu Structural Inertia, organisationalem Lernen und kognitiven Strukturen zur Ausbildung von Rigidität und Trägheit führen, nicht im Wi-

152 Beispielhaft seien an dieser Stelle Sunk Costs und Kommunikationsstrukturen (Structural Inertia), die Fokussierung auf Kernkompetenzen (Lerntrajektorien), selektive Wahrnehmung und Groupthink (kognitive Verfestigungen) sowie Netzeffekte (Pfadabhängigkeit) als Ursachen für organisationale Trägheit angeführt.

153 Vgl. hierzu die Ausführungen zu ,reaktiven Sequenzen' in Kapitel 2.3.1 dieser Arbeit.

154 Ein Lock-In zeichnet sich ebenfalls durch Kontinuität und Wandlungswiderstand aus. Vgl. hierzu die Definition von Trägheit in Kapitel 2.4 dieser Arbeit. 
derspruch zu einer pfadtheoretischen Erklärung von Trägheit. Im Gegenteil: Wie anhand der Typologie selbstverstärkender Mechanismen gesehen, bedient sich die Pfadtheorie eines breiten Kanons potentieller Quellen positiver Rückkopplungseffekte, auf die sich auch andere Theorien beziehen.

Beispielsweise führen sowohl die Theorie des Structural Inertia als auch die Pfadtheorie Sunk Costs im Sinne von Investitionen in Anlagen und Personal als Ursachen für organisationale Trägheit an. ${ }^{155} \mathrm{Im}$ Unterschied $\mathrm{zu}$ klaren UrsacheWirkungsbeziehungen zwischen Sunk Costs und Trägheit im Rahmen des Structural Inertia-Ansatzes, argumentiert die Theorie der Pfadabhängigkeit auf einer anderen, prozessualen Ebene. Pfadbedingte Trägheit auf Basis von Sunk Costs bildet sich erst durch das anschließende Entscheidungsverhalten aus. Sunk Costs stellen somit dasjenige Ereignis dar, welches den pfadabhängigen und zur Trägheit führenden Prozess auslöst. Erst die Entscheidung, eine bestimmte Strategie weiterzuverfolgen, weil bereits erhebliche Investitionen in die Ausgestaltung und Implementierung genau dieser Strategie getätigt wurden, formt die Prozesskette aus sich gegenseitig verstärkenden, miteinander verbundenen Entscheidungen. Das Resultat ist organisationale bzw. strategische Trägheit, ausgelöst durch Sunk Costs, jedoch zu erklären durch den Mechanismus der Selbstverstärkung.

In diesem Sinne liefert die Pfadtheorie eine alternative Erklärung zu Rigiditäten in Organisationen, die den Erklärungsansätzen der übrigen Konzepte nicht widerspricht, sondern diese in Teilen integriert und um eine non-lineare Prozessperspektive erweitert. So mag zwar das Sunk Costs-Argument ursächlich für Trägheit sein, aber diese ist nur dann pfadabhängigen Charakters, sofern die Sunk Costs einen positiven Rückkopplungsmechanismus bedingen. 
Hieran anschließend soll in dem nun folgenden Kapitel konkret auf die Konzeption strategischer Pfadabhängigkeit eingegangen werden. Hierzu wird ein zweischrittiges Modell entwickelt, das wiedergibt, wie sich das Konstrukt der strategischen Pfadabhängigkeit in das umfassende Feld von strategischer Rigidität eingliedert und somit eine spezielle, durch den Entstehungsprozess zu erklärende Ausprägung von Rigidität bzw. Trägheit darstellt. Ziel des Modells ist es, ein systematisches Diagnoseraster zur Analyse strategischer Pfadabhängigkeit bereitzustellen und dieses - wie im empirischen Teil unter Kapitel 4.4 vorgestellt wird - auf den Fall des Bertelsmann Buchclubs anzuwenden.

\subsection{Konzeption strategischer Pfadabhängigkeit}

Die empirische Analyse strategischer Pfadabhängigkeit basiert in der vorliegenden Arbeit auf zwei grundlegenden Schritten. Wie bereits erläutert, beschreibt das Phänomen der Pfadabhängigkeit durch den Lock-In eine spezifische Form von Rigidität, so dass vor der eigentlichen Pfaddiagnose zunächst der Nachweis von strategischer Rigidität erbracht werden muss.

Schritt 1 umfasst somit eine detaillierte Untersuchung des strategischen Entscheidungsverlaufs, um herauszufinden, ob sich der Verdacht auf strategische Rigidität erhärtet. Unter Verweis auf Kapitel 2.4 definieren sich Trägheit und Rigidität durch organisationale Beharrungstendenzen, obwohl eine Notwendigkeit zum Wandel vorliegt. ${ }^{156}$ Sofern sich eine Organisation unter stabilen Verhältnissen erfolgreich im Markt positioniert hat, liegt kein unmittelbarer Änderungsgrund vor, so dass Rigiditäten unauffällig und unbemerkt in Form von kontinuierlichen Entscheidungsprozessen bestehen können. Erst das Auftreten einer Änderungsnotwendigkeit bei gleichzeitiger strategischer Kontinuität enttarnt die tatsächliche Rigidität der Entscheidungstrajekto-

156 Vgl. Huff, J.O. et al. (1992) sowie Kapitel 2.4 dieser Arbeit. 
rie. Das Konstrukt der strategischen Rigidität lässt sich somit in zwei zentrale Elemente unterteilen:

Das Element ,strategische Kontinuität' bildet die gleichförmige, allerdings nur potentiell rigide Entscheidungstrajektorie ab, während mit dem zusätzlichen Element ,dynamische Umwelt' die Dringlichkeit des strategischen Wandels eingefangen werden soll. Unter dynamischen Marktbedingungen wird die strategische Flexibilität und somit die Fähigkeit, aus kontinuierlichen Entscheidungstrajektorien auszubrechen - zur kritischen Größe, um sich schnell ändernden Wettbewerbsfaktoren anzupassen, gegenzusteuern oder sogar proaktiv handeln zu können. Der Nachweis von strategischer Rigidität stützt sich somit auf die beiden Elemente ,strategische Kontinuität' und ,dynamische Umwelt'. Nachfolgende Tabelle 4 illustriert den soeben beschriebenen Schritt 1 der Pfadanalyse.

Tabelle 4: Rigiditätsnachweis als Schritt 1 der Pfadanalyse

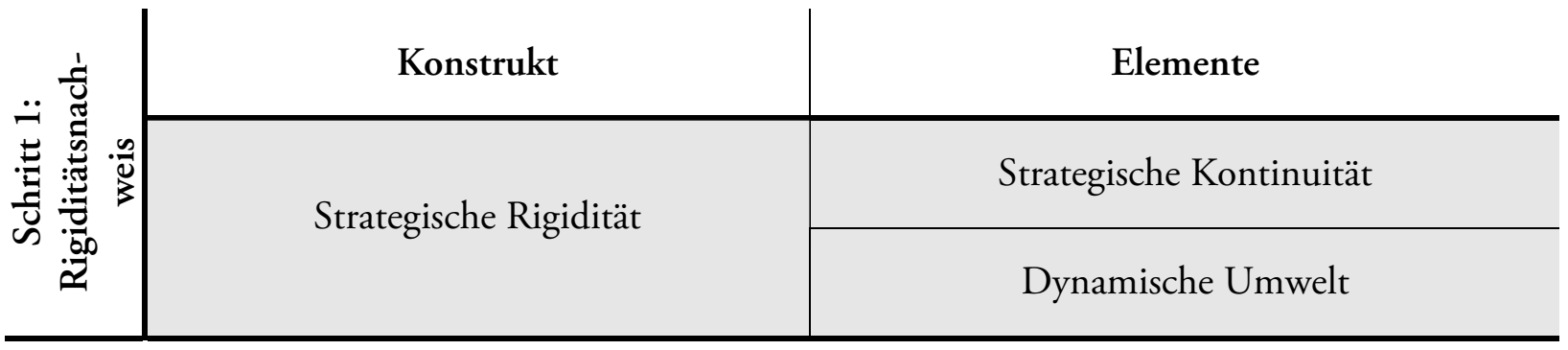

Quelle: Eigene Darstellung.

Sofern in Schritt 1 Rigidität nachgewiesen werden kann, gilt es in Schritt 2 herauszufinden, ob die strategische Rigidität auf pfadabhängigen Prozessen beruht, sich also durch selbstverstärkende Mechanismen ein Lock-In eingestellt hat, durch welchen der Befund der strategischen Rigidität zu erklären ist. Gemäß der einzelnen Phasen des Drei-PhasenModells der Pfadentstehung müssen hierzu vier Komponenten vorliegen: Historizität, Critical Juncture, positive Rückkopplung sowie der Lock-In. Ähnlich wie schon das Konstrukt der strategischen Rigidität weisen sämtliche Pfadkomponenten einen so hohen Abstrakti- 
onsgrad auf, dass deren Identifikation und Messung für die empirische Pfaddiagnose auf diesem Abstraktionsniveau unmöglich ist. Aus diesem Grund sollen die Komponenten auch in einzelne Elemente unterteilt werden, denen sich in einem zweiten Schritt (Kapitel 4.4.1) Indikatoren für die empirische Pfadanalyse zuordnen lassen.

Wie in Kapitel 2.3.2.1 ausführlich dargelegt, ist Phase I des Pfadentstehungsprozesses durch die zentrale Pfadkomponente der Historizität geprägt. Diese historische Verankerung von Entscheidungssituationen - unabhängig davon, ob sich ein Pfad herausbildet oder nicht - zeichnet sich durch eine ,eingeschränkte Kontingenz' aus. Auf der einen Seite ist der Entscheidungs- bzw. Handlungsspielraum in Phase I noch so weit geöffnet, dass eine Vielzahl von (strategischen) Entscheidungsverläufen möglich ist, auf der anderen Seite bedingt die strukturelle Vorprägung von Organisationen bereits eine leichte Einschränkung der Entscheidungsvarianz. Die Komponente „Historizität' in Phase I des Phasenmodells untergliedert sich somit in die beiden Elemente ,Kontingenz' und ,Vorprägung'.

Das Critical Juncture als zweite Pfadkomponente stellt dasjenige kritische Ereignis im Pfadprozess dar, welches die selbstverstärkenden Mechanismen auslöst und somit den eigentlichen Pfadentstehungsprozess einleitet. Ein Critical Juncture muss sich nicht zwangsweise auf nur ein wegweisendes Ereignis (z. B. eine Entscheidung) beziehen, sondern kann sich auch aus mehreren Ereignissen zusammensetzen, deren $\mathrm{Zu}$ sammenspiel die selbstverstärkenden Prozesse hervorruft. Die zu operationalisierenden Elemente der Pfadkomponente sind somit ,Small Events' (kleine Ereignisse). Hierbei ist zu beachten, dass das Attribut ,klein’ ein relativer Begriff und daher abhängig von der jeweiligen Referenzgröße ist. Zusätzlich ist die kritische Wirkung der kleinen Ereignisse vor dem Hintergrund der aktuellen pfadtheoretischen Forschung nur aus einer retrospektiven Betrachtung zu ermitteln - gerade weil zum Zeitpunkt des Eintretens noch keine kritische Auswirkung zu erahnen ist. 
Als dritte Komponente strategischer Pfadabhängigkeit ist der Mechanismus der positiven Rückkopplung in Phase II zu nennen. Bereits ausführlich in Kapitel 2.3.2.2 dargelegt, bietet die Literatur einen umfangreichen Katalog unterschiedlicher Formen von Selbstverstärkungsmechanismen an. Diese erheben jedoch nicht den Anspruch auf Vollständigkeit, sondern spiegeln den derzeitigen Forschungsstand wider. Eine theoriegeleitete Weiterentwicklung der unterschiedlichen Formen von Selbstverstärkungsmechanismen - hier als Elemente der Pfadkomponente ,positive Rückkopplung' bezeichnet - ist möglich; gleichwohl lässt sich der Katalog durch empirische Befunde ergänzen.

Durch die Wirkkraft der selbstverstärkenden Mechanismen wird schließlich der Lock-In als vierte Komponente und zugleich als Übergang zu Phase III der strategischen Pfadabhängigkeit erreicht. Hierzu benennt die Theorie der Pfadabhängigkeit das Element der ,potentiellen Ineffizienz'. Diese liegt vor, sofern sich eine gegenüber potentiellen Alternativen (ökonomisch) inferiore Strategieausrichtung eingestellt hat, deren Trajektorie nicht mehr verlassen wird. Als zweites Element zeichnet sich der Zustand des Lock-Ins durch die ,Immunität' des Systems gegenüber externer Beeinflussung und internem Wandel aus, da die pathologischen Strukturen der Pfadabhängigkeit ein Verlassen der strategischen Trajektorie nur schwer ermöglichen. Ob alternative Entscheidungswege nicht existieren, nicht wahrgenommen werden oder nicht wahrgenommen werden wollen, lässt sich schließlich durch das dritte Element der ,Ignoranz' näher beschreiben. Ignoranz indiziert, auf welcher Ebene (z. B. ressourcenbasiert oder kognitiv) die pfadabhängigen Kräfte wirken. Als viertes und letztes Element können zusätzlich ,stabilisierende Faktoren' auf die Aufrecherhaltung des LockIns Einfluss nehmen. Stabilisierende Faktoren wirken einseitig in einem linearen, nicht rekursiven bzw. verstärkenden Verhältnis auf strategische Entscheidungsprozesse ein, können den Lock-In unterstützen, jedoch nicht als alleinige Ursache für dessen Entstehung angesehen werden. 
Eine Übersicht über die einzelnen Elemente dieses zweiten Schritts der Pfadanalyse - der Pfaddiagnose - sowie deren Eingliederung in das Drei-Phasenmodell der Pfadentstehung - veranschaulicht nachfolgende Tabelle 5.

Tabelle 5: Pfaddiagnose als Schritt 2 der Pfadanalyse

\begin{tabular}{|c|c|c|c|}
\hline \multirow{8}{*}{ 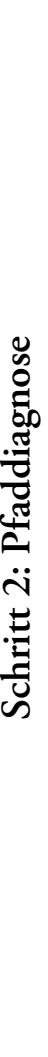 } & & Komponenten & Elemente \\
\hline & 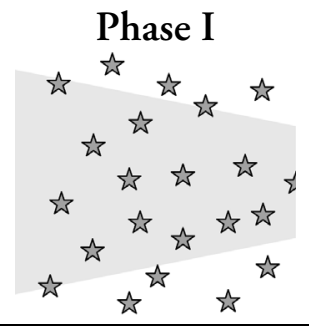 & Historizität & Kontingenz \\
\hline & & Critical Juncture & Small Events \\
\hline & Phase II & Positive Rückkopplung & $\begin{array}{l}\text { Selbstverstärkende } \\
\text { Mechanismen }\end{array}$ \\
\hline & \multirow{2}{*}{ 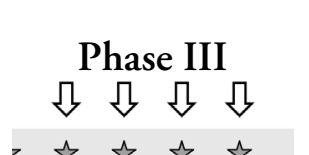 } & \multirow{4}{*}{ Lock-In } & Ineffizienz \\
\hline & & & Immunität \\
\hline & \multirow{2}{*}{ 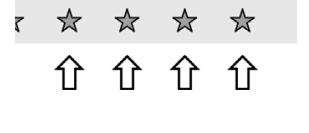 } & & Ignoranz \\
\hline & & & Stabilisierende Faktoren \\
\hline
\end{tabular}

Quelle: Eigene Darstellung.

Sämtliche Elemente aus Schritt 1 und Schritt 2 der soeben vorgestellten Pfadanalyse werden im empirischen Teil unter Kapitel 4.4 .1 in messbare Indikatoren überführt, um diese dann anhand der strategischen Entwicklung des Bertelsmann Buchclubs von 1950 bis 2007 erheben zu können.

Bevor mit der empirischen Pfadanalyse begonnen wird, muss jedoch zuvor geklärt werden, auf welches methodische Vorgehen sich die Pfadanalyse stützt. Hierzu sollen im nun folgenden Kapitel das Forschungsdesign, die Erhebungs- sowie Auswertungsmethoden diskutiert werden. 


\section{Der Fallstudienansatz als Forschungsstrategie}

Um die soeben vorgestellte Konzeption zur Pfadanalyse empirisch anzuwenden, ist ein adäquates methodisches Instrumentarium notwendig. Bei dessen Auswahl muss vor allem die Forschungsfrage, warum eine Organisation langfristig an verlustreichen Strategien festhält, Berücksichtigung finden. Die anzustellende Untersuchung beschäftigt sich also mit der Trägheit von Unternehmen und sucht konkret nach den Ursachen für strategische Beharrungstendenzen. Zur Beantwortung dieser Frage soll die Pfadtheorie - übertragen auf strategische Unternehmensentscheidungen - herangezogen werden.

Aufgrund des Prozesscharakters der Phänomene ,Trägheit' sowie ,Pfadabhängigkeit' muss das Forschungsdesign der Anforderung gerecht werden, unternehmensstrategische Entscheidungen im Zeitverlauf messen zu können. Zweitens kommt hinzu, dass die Datenanalyse detaillierten Aufschluss über strategische Zusammenhänge und kausale Verknüpfungen geben soll. Beide Anforderungen vermag der Fallstudienansatz zu erfüllen, so dass dessen Auswahl als Forschungsstrategie erfolgte. ${ }^{157}$ Die Begründung hierfür sowie das genaue methodische Vorgehen werden in den folgenden Kapiteln ausführlich diskutiert.

\subsection{Fallstudien und ihre Abgrenzung zu alternativen Forschungsstrategien}

Da in der Literatur zum Terminus ,Fallstudie' kein einheitliches Begriffsverständnis vorliegt, muss zunächst das dieser Arbeit zugrunde liegende Verständnis erörtert werden.

Einleitend lässt sich bereits auf eine zentrale Unterscheidung hinweisen: Fallstudien werden häufig forschungsmethodisch im Sinne eines konkreten Instruments zur Datenerhebung aufgefasst. In der Sozialforschung wird der Begriff der Fallstudie bei-

157 Vgl. George, A.L./Bennett, A. (2005), S. 19-22; Siggelkow, N. (2007), S. 20-24. 
spielsweise oft mit der Erhebungsmethode der ,teilnehmenden Beobachtung' gleichgesetzt. Im Gegensatz dazu lassen sich Fallstudien in einem abstrakten Begriffsverständnis als allgemeine Forschungsstrategie auffassen. ${ }^{158}$ Letzterem Verständnis folgt auch die vorliegende Arbeit, indem die teilnehmende Beobachtung als eines von mehreren möglichen Instrumenten der Datenerhebung in den Fallstudienansatz als übergeordnete Einheit integriert wird. In diesem Sinne versucht die Fallstudie als grundlegende Forschungsstrategie, durch ein möglichst umfassendes Spektrum von Erhebungs- und Analysemethoden Antworten auf die forschungsleitende(n) Frage(n) zu finden. ${ }^{59}$

Vor allem im Gegensatz zu quantitativen Forschungsansätzen sehen sich Fallstudien häufig einer erhöhten Kritik ausgesetzt. Kritische Argumente beziehen sich im Kern auf die mangelnde Quantifizierbarkeit, fehlende Objektivität sowie die begrenzte Aussagekraft zur Generalisierung. ${ }^{160}$ Angesichts dieser grundlegenden Kritikpunkte ist es notwendig, eine differenzierte Sichtweise auf den Forschungsgegenstand einzunehmen. Dies erfordert die Auseinandersetzung mit der Art des zu untersuchenden Phänomens sowie des übergeordneten Forschungsziels. Weder Fallstudien noch alternative Ansätze wie Beobachtung oder Umfrage lassen sich pauschal beurteilen, sondern sämtliche Ansätze müssen vor dem Hintergrund des jeweiligen Forschungskontextes bewertet werden. Der Forschungsaufbau muss mit dem Forschungsgegenstand bzw. -ziel übereinstimmen.

Vielfach werden Fallstudien der explorativen Forschung zugeordnet. Explorative Untersuchungen tasten eher intuitiv ein zu untersuchendes Feld ab, ohne vorher konkrete Forschungsfragen bzw. zu testende Hypothesen aufgestellt zu haben. Im Gegenteil: Das Ziel explorativer Forschung liegt häufig in der Generierung konkreter Hypothesen, die anschließend im Rahmen von deskriptiven bzw. erklärenden Forschungsdesigns überprüft werden sollen. Grundsätzlich ist explorative Forschung mit dem

\footnotetext{
158 Vgl. Hamel, J. (1992), S. 1.

159 Vgl. Hamel, J. (1992), S. 1-7.

160 Vgl. Siggelkow, N. (2007), S. 20-24.
} 
Ansatz der Fallstudie möglich, allerdings ist dies nur ein Teilbereich des vollständigen Potentials von Fallstudien. Mit Hilfe von Fallstudien lassen sich ebenso Phänomene beschreiben, um Theorien zu testen und weiterzuentwickeln bzw. neue Theorien zu generieren. ${ }^{161}$ In diesem Sinne ist von einer chronologischen Kategorisierung von Fallstudien abzusehen, die den Fallstudienansatz eher einer explorativen Vorstudie zuordnet, deren Ergebnisse in Form von zu testenden Hypothesen alternativen Forschungsdesigns überlassen wird. Fallstudien stehen somit gleichwohl auf einer Ebene mit weiteren Forschungsstrategien wie z. B. dem Experiment oder der Umfrage. ${ }^{162}$

Zur genaueren Beschreibung des Fallstudienansatzes ist es hilfreich, eine Abgrenzung zu alternativen Ansätzen vorzunehmen. In Anlehnung an YIN (2003) sowie ergänzend HaMMERSLEY/GOMM (2000) lässt sich folgende Matrix aufspannen, welche im Anschluss eingehend erläutert wird.

Tabelle 6: Abgrenzung der Fallstudie von alternativen Forschungsansätzen

\begin{tabular}{l|l|l|l} 
& Fallstudie & Umfrage & Experiment \\
\hline $\begin{array}{l}\text { Form der } \\
\text { Forschungsfragen }\end{array}$ & $\begin{array}{l}\text { Wie? } \\
\text { Warum? }\end{array}$ & $\begin{array}{l}\text { Wer? Was? Wo? } \\
\text { Wie viel? }\end{array}$ & $\begin{array}{l}\text { Wie? } \\
\text { Warum? }\end{array}$ \\
\hline $\begin{array}{l}\text { Kontext-I } \\
\text { Verhaltenskontrolle }\end{array}$ & Nein & Nein & Ja \\
\hline Fallzahl & Gering & Hoch & Gering \\
\hline $\begin{array}{l}\text { Anzahl der analysier- } \\
\text { ten Variablen pro Fall }\end{array}$ & Hoch & Gering & Gering \\
\hline $\begin{array}{l}\text { Art des } \\
\text { Forschungsziels }\end{array}$ & $\begin{array}{l}\text { Qualifizierung } \\
\text { von Daten }\end{array}$ & $\begin{array}{l}\text { Quantifizierung } \\
\text { von Daten }\end{array}$ & $\begin{array}{l}\text { Quantifizierung } \\
\text { von Daten }\end{array}$ \\
\hline
\end{tabular}

Quelle: Eigene Darstellung in Anlehnung an Yin, R.K (1994), S. 6; Hammersley, M./ Gomm, R. (2000), S. 4.

161 Vgl. George, A.L./Bennett, A. (2005), S. 109-124; Blumberg, B. et al. (2005), S. 191.

162 Vgl. Blumberg, B. et al. (2005), S. 190. 
Zunächst hängt die Wahl eines passenden Forschungsansatzes von der Form der zu klärenden Forschungsfragen ab. Während sich eine Umfrage dazu eignet, die Häufigkeit des Auftretens eines bestimmten Phänomens (Wie viel?) oder die Ausprägung eines Phänomens (Wer? Was?) zu ermitteln, ${ }^{163}$ zielen sowohl die Fallstudie als auch das Experiment auf die Beantwortung von Wie- und Warum-Fragen ab. ${ }^{164}$ Grund hierfür ist, dass beide Forschungsansätze eine detaillierte Beobachtung von Elementen im Zeitablauf ermöglichen und somit über die Erfassung von Elementen und deren Verbreitung hinausgehen.

Das zweite Unterscheidungskriterium bezieht sich auf die Kontrollierbarkeit der Kontextfaktoren. Beim Experiment hat der Forscher die Möglichkeit, das Verhalten der beteiligten Akteure zu manipulieren, um so beispielsweise die Wirkung einer abhängigen Variablen auf eine unabhängige unter Ausschluss sämtlicher Störfaktoren zu untersuchen. ${ }^{165}$ Diese Möglichkeit der Manipulation eröffnet sich weder bei der Umfrage noch bei der Fallstudie. In beiden Forschungsansätzen werden die zu untersuchenden Phänomene unter natürlichen Bedingungen studiert. Die Grenzen zwischen fokalem Untersuchungsgegenstand und Kontextfaktoren sind dabei nicht immer klar erkennbar. ${ }^{166}$

Bezüglich des dritten Differenzierungskriteriums - der Höhe der Fallzahl - unterscheidet sich die Umfrage von Experiment und Fallstudie. Ersterer Forschungsansatz ermöglicht es und zielt darauf ab, eine hohe Fallzahl in die Datenbasis zu integrieren, während sowohl in der Fallstudie als auch beim Experiment mit einer vergleichsweise geringen Anzahl von Fällen ausgekommen werden muss. ${ }^{167}$ Nicht selten - wie auch in der vorliegenden Arbeit - beträgt die Fallzahl die Größe eins.

\footnotetext{
163 Vgl. Yin, R.K. (1994), S. 6.

164 Vgl. Yin, R.K. (1994), S. 7.

165 Vgl. Yin, R.K. (1994), S. 8.

166 Vgl. Hartley, J. (2004), S. 323; Yin, R.K. (1994), S. 13.

167 Vgl. Hammersley, M./Gomm, R. (2000), S. 4.
} 
Als Ausgleich für die geringe Fallzahl erlaubt es der Fallstudienansatz, das Auftreten und die Veränderung vieler unterschiedlicher Variablen zu betrachten, wohingegen Umfrage und Experiment auf eine geringere Anzahl limitiert sind. ${ }^{168}$

Schließlich unterscheidet sich die Fallstudie von Experiment und Umfrage durch das übergreifende Forschungsziel: Fallstudien können und sollen keine Quantifizierung von Daten liefern. Generalisierungen und Rückschlüsse auf die Grundgesamtheit - wie bei Experiment und Umfrage - lassen sich nicht vornehmen.

Anhand der vergleichenden Gegenüberstellung lässt sich speziell für den Fallstudienansatz folgende Definition zusammenfassend festhalten:

Eine Fallstudie ist eine empirische Untersuchung, die

- Antworten auf Forschungsfragen mit Wie- und Warum-Charakter sucht,

- ein Phänomen in einem realweltlichen Kontext untersucht, dessen Grenzen nicht immer klar erkennbar sind,

- bei einer geringen Anzahl von Fällen viele Variablen in Betracht zieht,

- keine empirische Generalisierung anstrebt.

\subsection{Grundtypen eines Fallstudienaufbaus}

Nach der definitorischen Abgrenzung des Fallstudienansatzes soll in einem nächsten Schritt auf die genauere Ausgestaltung des Fallstudienaufbaus eingegangen werden. Hierzu wird zunächst der in der vorliegenden Arbeit zur Anwendung kommende Grundtypus der holistischen Einzelfallstudie in Abgrenzung zu alternativen Grundtypen erörtert, um in Kapitel 3.4 auf die konkrete Ausgestaltung des Fallaufbaus zur Analyse des Bertelsmann Buchclubs zu sprechen zu kommen.

In Anlehnung an YIN (1994) stehen prinzipiell vier unterschiedliche Fallstudientypen zur Verfügung:

Vgl. Hammersley, M./Gomm, R. (2000), S. 4. 
Fallstudien können einerseits als Einzel- oder Mehrfallstudien und andererseits nach Anzahl der Analyseobjekte - als holistische oder integrierte Fallstudien konzipiert werden. ${ }^{169}$ Der für die vorliegende Arbeit relevante Typ Nummer eins ist die holistische Einzelfallstudie, welche ein einzelnes Analyseobjekt mit angrenzendem Kontext innerhalb eines Einzelfalls untersucht. Im Gegensatz dazu integriert der zweite Typus - die holistische Mehrfallstudie - mehrere holistische Fälle innerhalb einer Fallstudienuntersuchung. Im Rahmen einer integrierten Einzelfallstudie als Typ drei werden innerhalb eines Falls (z. B. einer Firma) mehrere Analyseobjekte (z. B. Abteilungen) erforscht. Dessen Ausweitung auf mehrere Fälle beschreibt die integrierte Mehrfallstudie als vierter Fallstudientypus. ${ }^{170}$

Abbildung 8: Grundtypen eines Fallstudienaufbaus
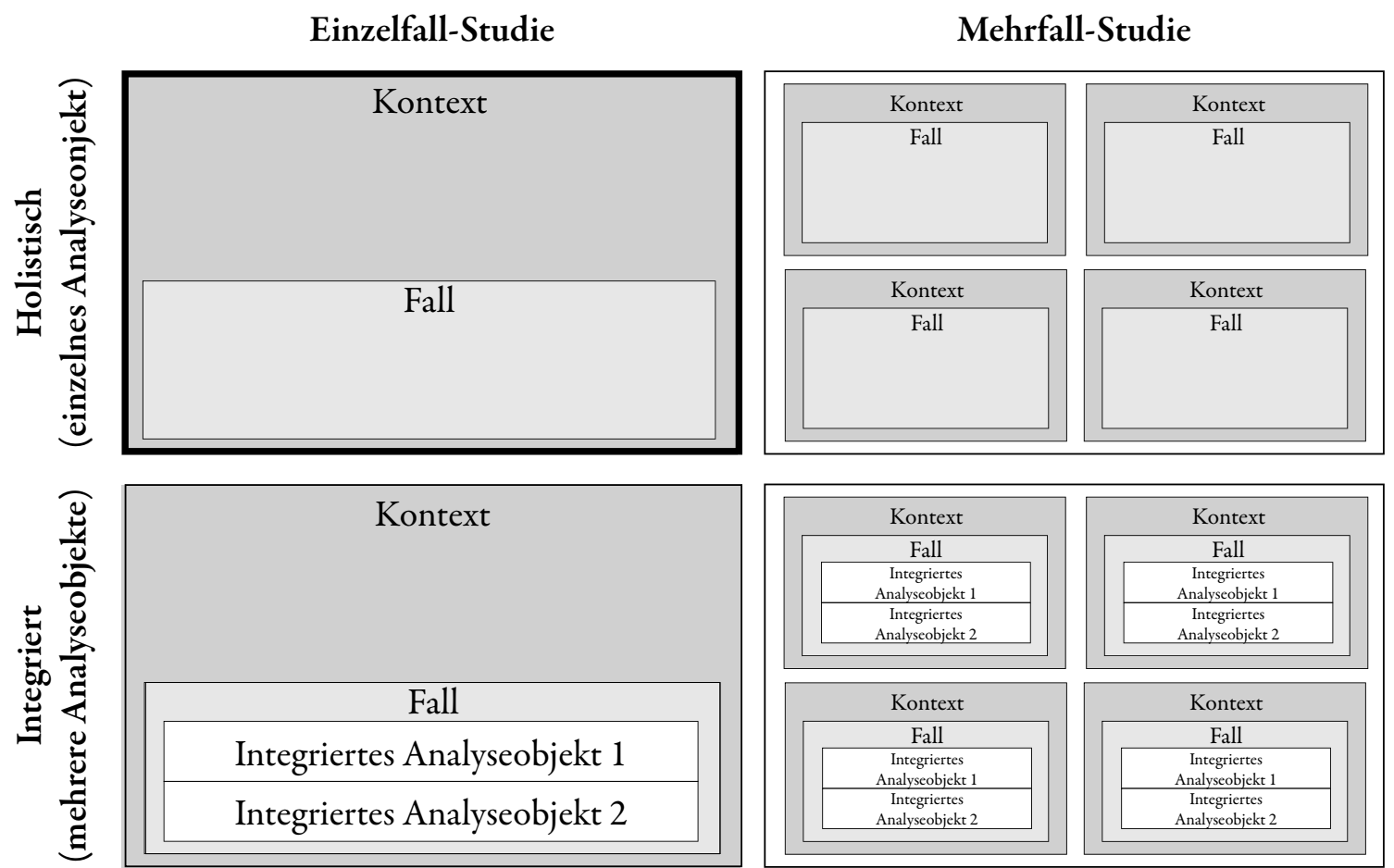

Quelle: Schmidt, L. (2006), S. 113; Yin, R.K. (1994), S. 39.

169 Vgl. Yin, R.K. (1994), S. 38.

170 Vgl. Yin, R.K. (1994), S. 38-53 ; Vaus, de, D. (2003), S. $220 f$. 


\subsubsection{Fall, Kontext und Analyseobjekt}

Anhand der Fallstudientypologisierung wird deutlich, dass ein wesentliches Abgrenzungskriterium die Unterscheidung nach Fall, Kontext sowie Analyseobjekt ist. Nach dieser Kategorisierung zählen sowohl diejenigen Objekte, die für die Beantwortung der Forschungsfrage unmittelbar (Fall) als auch mittelbar (Kontext) relevant sind, zum Gesamtaufbau der Fallstudie. Objekte, die weder unmittelbar noch mittelbar die Forschungsfrage betreffen, werden nicht in der Fallstudie erfasst. Ein Fall kann eine gesamte Organisation umfassen, möglich ist jedoch auch die Betrachtung von Projektteams, die sich aus organisationsinternen und -externen Mitgliedern zusammensetzen. Ein Fall ist somit diejenige organisatorische Einheit, innerhalb derer das zu untersuchende Phänomen, das Analyseobjekt, abläuft. ${ }^{171}$ Sofern sich innerhalb eines Falles mehrere zu unterscheidende Einheiten befinden, deren separate Betrachtung zur Klärung der Forschungsfrage beiträgt, empfiehlt sich die Aufteilung des Falls in mehrere Analyseobjekte im Rahmen eines integrierten Fallstudienaufbaus. ${ }^{172}$ Die Bestimmung des relevanten Kontexts für den zu untersuchenden Fall ist insofern von großer Bedeutung, als dass er wie der Fall gleichermaßen in die Untersuchung mit einfließt. Häufig lassen sich die Analyseobjekte der Fallstudie erst durch die Hinzuziehung der Kontextfaktoren begreifen, da diese direkten bzw. indirekten Einfluss auf die fokalen Phänomene der Fallstudie ausüben. Welche Kontextfaktoren für den Untersuchungsgegenstand relevant sind und welche außer Acht gelassen werden können, stellt eine große Herausforderung an den Forscher dar. Relevante Faktoren lassen sich nicht selten erst in einem fortgeschrittenen Forschungsstadium ermitteln, so dass der Forschungsaufbau in einem iterativen Prozess angepasst werden muss.

171 Vgl. Vaus, de David (2003), S. 220.

172 Vgl. Yin, R.K. (1994), S. 41f. 


\subsubsection{Einzel- und Mehrfallstudie}

Neben der Abgrenzung von Fall, Kontext und Analyseobjekten umfasst die Bestimmung eines geeigneten Fallstudienansatzes ebenfalls die Auswahl der Fallzahl. Die Konstituierung einer Einzelfallstudie bietet sich unter folgenden Szenarien an:

Erstens ist das Design einer Einzelfallstudie geeignet, sofern es gilt, die Annahmen und Rahmenbedingungen einer bestehenden Theorie zu testen. In diesem Fall dient die Fallstudie als ,kritischer Fall', der die Funktion erfüllt, Theorien zu testen, zu widerlegen oder zu erweitern. Eine zweite Situation bezieht sich auf extreme oder einzigartige Situationen, die bislang unerforscht sind und Ausnahmen darstellen. In diesem Fall ist die Einzelfallstudie als, extremer Fall' angelegt. Drittens eignet sich die Einzelfallstudie zur Erforschung von neuartigen Analyseobjekten, zu denen bislang kein $\mathrm{Zu}$ gang bestand und die somit der Wissenschaft verschlossen waren. Es liegt ein ,enthüllender Fall' vor. ${ }^{173}$ In allen drei Fällen ist der Forschungsaufbau einer Einzelfallstudie besonders geeignet, sofern diese als Langzeitstudie angelegt ist. Das Design der Einzelfallstudie ist darauf ausgelegt, zu untersuchende Phänomene intensiv und detailliert zu beobachten und vermag somit Entwicklungen im Zeitverlauf verlässlich einzufangen.

Im Gegensatz dazu lassen sich Situationen anführen, unter denen Mehrfallstudien der geeignete Weg zur Erreichung des Forschungsziels sind. Ein zentraler Grund dafür liegt in der Möglichkeit der Replikation. Sofern in einem zweiten Fall die gleichen oder ähnliche Ergebnisse wie in dem ersten Fall reproduziert werden können, lässt sich von literarischer Replikation sprechen. Theoretische Replikation liegt vor, wenn der zweite Fall ein (vorhersagbar) widersprüchliches Ergebnis hervorbringt. Lässt sich durch Replikation die Bestätigung bestimmter theoretischer Annahmen empirisch untermauern, so darf deren Logik nicht mit einer Generalisierbarkeit im Sinne der quantitativen Datenerhebung verwechselt werden. Wie schon bei der Einzelfallstudie

173 Vgl. Yin, R.K. (1994), S. 38-41; Blumberg et al. (2005), S. 192f.; Gomm, R. et al. (2000), S. 106-108. 
lässt auch der Forschungsaufbau der Mehrfachstudie keine Rückschlüsse auf die ,Grundgesamtheit' anderer Fälle zu.

Bevor unter Kapitel 3.4 auf die konkrete Ausgestaltung des dieser Arbeit zugrunde liegenden Fallstudiendesigns eingegangen wird, sollen zunächst die Gütekriterien, anhand derer sich der Fallstudienansatz messen lässt, vorgestellt werden.

\subsection{Qualitätsansprüche an die Fallstudienforschung}

In den vorangegangenen Kapiteln wurden bereits die Möglichkeiten und Grenzen des Fallstudienansatzes angesprochen. Der Nachteil mangelnder Quantifizierbarkeit der Daten wiegt sich insofern auf, als dass der Forscher durch die Art und Tiefe der Daten die Möglichkeit hat, die beobachteten Vorgänge zu deuten und ausführlich zu erschließen. Gerade durch die Leistung des Forschers, die erhobenen, nur selten quantifizierbaren Daten zu verstehen, sieht sich der Fallstudienansatz einer erhöhten Kritik und Angreifbarkeit ausgesetzt. Daher ist es besonders wichtig, feste Qualitätskriterien zu bestimmen, anhand derer sich die Güte der Fallstudienforschung messen lässt. Im Folgenden soll auf diese Kriterien der Konstrukt-Validität, der internen und externen Validität sowie der Reliabilität eingegangen werden. ${ }^{174}$

Konstrukt-Validität liegt vor, sofern die Messung der operationalisierten Indikatoren auf das dahinter liegende Konstrukt schließen lässt. Aufgrund der Interpretationsleistung des (Fallstudien)Forschers ist dieser besonders häufig dem Vorwurf mangelnder Objektivität ausgesetzt. Aus diesem Grund muss vor allem auf zwei Dinge geachtet werden: Zum einen muss eine klare Eingrenzung und Benennung des zu untersuchenden Forschungsgegenstandes erfolgen, der sich auf die Forschungsfrage bezieht. Zum zweiten müssen die konkreten, zu messenden Indikatoren systematisch und

174 Vgl. Yin, R.K. (1994), S. 33. 
sorgfältig aus dem Untersuchungsgegenstand abgeleitet werden und somit in der Lage sein, die zu erforschenden Phänomene zu beschreiben.

Interne Validität bezieht sich vornehmlich auf Fallstudiendesigns, die darauf ausgelegt sind, kausale Zusammenhänge zu erforschen. Sofern aus den Forschungsergebnissen kausale Zusammenhänge abgeleitet und alternative Erklärungen ausgeschlossen werden können, liegt interne Validität vor. Eine intern valide Fallstudie muss daher sämtliche relevanten Einflussfaktoren in der Untersuchung beachten. ${ }^{175}$

Im Gegensatz zu interner Validität erhebt das Qualitätskriterium der externen Validität den Anspruch der Generalisierbarkeit auch über den konkreten Fall hinaus. Ähnlich wie bei der Umfrage wird so auch an Fallstudien die Forderung nach einer statistischen Generalisierbarkeit herangetragen. Diesen Anspruch kann und will die Fallstudienforschung jedoch gar nicht erfüllen, liegt ihr Grundverständnis doch weniger in der statistischen als viel mehr in der analytischen Generalisierbarkeit. Wie bereits im vorangegangenen Kapitel 3.1 angedeutet, wollen Fallstudien nicht auf andere Fälle generalisieren, sondern die Erkenntnisse zu dem darüber liegenden theoretischen Verständnis replizieren. Die verwendete Theorie wird durch empirische Beobachtungen angereichert. ${ }^{176}$

Schließlich müssen auch Fallstudien dem vierten und letzten Qualitätskriterium der Reliabilität gerecht werden. Im Kern ist eine Untersuchung reliabel, sofern die Messwerte der Fallstudie bei wiederholten und parallelen Messungen unter sonst gleichen Rahmenbedingungen stabil bleiben. Da sich die wiederholte Messung in vielen Fällen kaum umsetzen lässt, ist es wichtig, eine möglichst exakte und intersubjektiv nachvollziehbare Dokumentation über das Forschungsvorgehen zu erstellen. So ist zumindest potentiell die Rekonstruktion des Forschungsprozesses möglich.

\footnotetext{
175 Vgl. Vaus, de D. (2003), S. 233.

176 Vgl. Vaus, de D. (2003), S. 237f.
} 
Anhand der einzelnen Qualitätskriterien von Fallstudien werden auch die pfadanalytischen Ergebnisse der vorliegenden Arbeit kritisch gewürdigt. Die entsprechende Diskussion dazu erfolgt in Kapitel 5.2.

Im nun folgenden Kapitel werden allerdings zunächst die in Kapitel 3.2 vorgestellten Bausteine des Fallstudienansatzes auf die zu beantwortende Forschungsfrage zugeschnitten. Nach der Begründung zur Auswahl des Fallstudienansatzes erfolgt eine genaue Beschreibung des konkreten Fallstudiendesigns.

\subsection{Bertelsmann Buchclub: Eine holistische Einzelfallstudie}

Der Forschungsansatz einer Fallstudie ist grundsätzlich zur Analyse (dem Nachweis und der Ursachenerforschung) von strategischer Rigidität in Organisationen geeignet.

Unter Verweis auf die Definition von Fallstudien in Kapitel 3.1 ermöglichen Fallstudien die Beantwortung von Warum-Fragen, indem sie eine detaillierte Erfassung von $\mathrm{Zu}$ sammenhängen und kausalen Verknüpfungen im Zeitablauf ermöglichen. Hierbei ist keine empirische Generalisierung angestrebt, sondern es wird das Ziel verfolgt, die bestehenden Annahmen der Pfadtheorie empirisch zu testen. Wie ausführlich in der Theoriediskussion zur Pfadabhängigkeit erörtert, zeichnet sich die Theorie der Pfadabhängigkeit durch ihren Prozesscharakter aus. Notwendige Bedingung von Pfadprozessen ist der Mechanismus der positiven Rückkopplung, über welchen die einzelnen Elemente der Prozesskette miteinander verknüpft sind. Zur Erforschung dieser kausalen Verknüpfungen eignet sich der Forschungsaufbau einer Fallstudie besonders: „A final advantage of case studies is their ability to accomodate complex causal relations such as equifinality, complex interactions effects, and path dependency. " ${ }^{177}$ Umfragen mit hohen Fallzahlen vermögen zwar Korrelationen zwischen einzelnen Variablen herauszufinden; um Kausali-

177 George, A.L./Bennett, A. (2005), S. 22. 
täten und die Art der Wirkrichtung der ermittelten Zusammenhänge zu verstehen, ist jedoch das Fallstudiendesign die geeignete Forschungsstrategie. ${ }^{178}$

Nach der Festlegung der Fallstudie als grundlegenden Forschungsansatz muss in einem zweiten Schritt die konkrete empirische Fallauswahl getroffen werden. Im Gegensatz zu quantitativen Untersuchungen erfolgt die Fallbestimmung nicht zufällig, sondern wird bewusst durch das zu erforschende Phänomen gesteuert. ${ }^{179}$ Kriterien für die Fallauswahl der vorliegenden Arbeit waren fundierte Hinweise auf strategische Pfadabhängigkeit in einer Organisation. Diese Bedingung erfüllte die strategische Entwicklung des Bertelsmann Buchclubs. Seit seiner Gründung 1950 erfolgte bis zum Zeitpunkt der ersten Datensichtung 2005 kein fundamentaler Wandel. Gleichzeitig ließen sich vor allem von 1992 an signifikante Mitgliedereinbrüche und Verluste feststellen, und der Buchmarkt erwies sich als sehr dynamisch und kompetitiv. Die Indizien der strategischen Kontinuität bei gleichzeitigen wirtschaftlichen Verlusten und dynamischen Marktbedingungen ließen die Vermutung auf strategische Trägheit zu. Diese Trägheit sollte durch die Annahmen der Pfadtheorie dahingehend überprüft werden, ob sich die strategischen Beharrungstendenzen durch die Pfadtheorie erklären lassen.

Ausgehend von dem zu erforschenden Phänomen strategischer Pfadabhängigkeit auf Organisationsebene wurde das Fallstudiendesign auf eine Einzelfallstudie eingegrenzt. In diesem Sinne wurde die strategische Entwicklung des Bertelsmann Buchclubs als kritischer Fall angelegt, anhand dessen sich die Theorie der Pfadabhängigkeit erproben ließ. Nach Festlegung der Forschungsstrategie (Fallstudie) und Bestimmung der organisatorischen Einheit (Buchclub als Einzelfall) galt es in einem nächsten Schritt, eine Trennung zwischen Analyseobjekt und Kontextfaktoren vorzunehmen. Als fokales Analyseobjekt wurden die strategischen Entscheidungsverläufe des Buch-

178 „As statistical researchers frequently point out, correlation does not imply causation.“ George, A.L/Bennett, A. (2005), S. 21.

179 Vgl. Vaus, de D. (2003), S. 238f. 
clubs festgelegt, die über den zu erfassenden Zeitraum von 1950 bis 2007 untersucht werden sollten. Gemäß der Definition von strategischen Entscheidungen galt es, die beobachtbaren strategischen Entschlüsse (als Ergebnis von bewusst bzw. unbewusst ablaufenden Entscheidungsprozessen) im Bertelsmann Buchclub abzubilden. Die Tatsache, dass sich im Laufe des Analysezeitraums auf Basis des Buchclubs zahlreiche weitere Sparten, Divisionen und eigenständige Firmen im In- und Ausland herausgebildet haben, erforderte eine klare Abgrenzung von fokalem Untersuchungsgegenstand, Kontextfaktoren sowie nicht verwandter Umwelt.

Während die strategische Ausrichtung des deutschen Bertelsmann Buchclubs ein klar umrissenes Analysefeld ergab, musste die Bestimmung der Grenzen zwischen Kontext und weiterer Umwelt im Laufe der Datenerhebung angepasst werden. Als relevante Kontextfaktoren wurden schließlich die Druckerei- und Logistikbetriebe sowie die Eigenverlage des Bertelsmann-Konzerns ermittelt, da diese in direkter Beziehung auf die strategische Entwicklung des Buchclubs Einfluss nahmen. Gleichermaßen flossen die ausländischen Bertelsmann Buchclubs als relevante Einflussfaktoren des Kontexts mit in die Analyse ein. Schließlich wurde die Gesamtheit der externen, für den Bertelsmann Buchclub tätigen Vertriebsfirmen als Kontextfaktor in die Analyse der Fallstudie integriert. Diese befinden sich zwar organisatorisch außerhalb des Systems Buchclub, unterhalten jedoch entscheidende Austauschbeziehungen mit dem Club und stellen somit relevante Faktoren der verwandten Umwelt als Kontext dar.

Zusammenfassend lässt sich folgender Forschungsaufbau festhalten: Die Forschungsfrage, warum eine Organisation langfristig an verlustreichen Strategien festhält, wird im Rahmen einer holistischen Einzelfallstudie untersucht. Ziel ist die empirische Anwendung der zentralen Annahmen der Pfadtheorie. Die fokale Analyseeinheit ist der strategische Entscheidungsverlauf des deutschen Bertelsmann Buchclubs von 1950 bis 2007. Es handelt sich daher um eine retrospektive Längsschnittstudie. Als relevante Kontextfaktoren fließen die organisatorischen Einheiten der Druckerei-, 
Logistikbetriebe und Eigenverlage, die konzerneigenen, ausländischen Buchclubs sowie die externen Vertriebsfirmen mit in die Analyse ein.

\subsection{Datenerhebung und -auswertung}

Nachdem die genaue Ausgestaltung des vorliegenden Fallstudienaufbaus ausführlich dargelegt wurde, soll im nun folgenden Kapitel auf die Methodik der Datenerhebung und -auswertung eingegangen werden. Forschungsleitend waren die von YIN (1994) vorgeschlagenen Prinzipien der Datenerhebung, um die Qualität der empirischen Untersuchung zu optimieren. Die einzelnen Prinzipien - multiple Datenquellen, eine Fallstudiendatenbank sowie die genaue Nachvollziehbarkeit der Argumentation werden nun anhand der Fallstudie des Bertelsmann Buchclubs diskutiert.

\subsubsection{Multiple Datenquellen und Triangulation}

In Anlehnung an Yin (1994) sowie Blumberg (2005) lassen sich Datenquellen grob zu drei Kategorien zusammenfassen: Dokumentationen und Archivdaten, Interviews, sowie (nicht)teilnehmende Beobachtung. Zur Erhöhung der Verlässlichkeit sollte sich die Datenlage nicht auf einzelne Datenquellen beschränken, sondern auf ein möglichst breites Spektrum unterschiedlicher Quellen zurückgreifen. ${ }^{180}$ Da jedoch nicht jede Datenquelle für jedes Untersuchungsdesign geeignet ist, muss der prinzipielle Anspruch einer möglichst breiten Datenbasis vor dem Hintergrund des konkreten Untersuchungsdesigns relativiert werden.

Der in der vorliegenden Arbeit zu untersuchende Analysezeitraum umfasst die Jahre 1950 bis 2007 und entzieht sich somit der Datenerfassung aktueller Ereignisse. Als Konsequenz scheidet die Methodik der teilnehmenden bzw. nicht teilnehmenden Beobachtung aus, da das zu untersuchende Analyseobjekt - die strategische Entwicklung des Buchclubs - in der Vergangenheit liegt. Die Datenquellen in der vorliegen- 
den Arbeit basieren demzufolge auf Dokumentationen und Archivdaten sowie Interviews. Zur genaueren Kategorisierung des Datenmaterials sowie einer kritischen Quellenprüfung wurde eine Einordnung in ein Kommunikationsmodell vorgenommen (Abbildung 9).

Als Leitfaden für die Datenerhebung war zwar der jeweilige inhaltliche Textgegenstand maßgeblich, allerdings wurden bei sämtlichen Inhalten sowohl Quelle und Medium als auch die Rezipienten berücksichtigt.

Abbildung 9: Einfaches inhaltsanalytisches Kommunikationsmodell

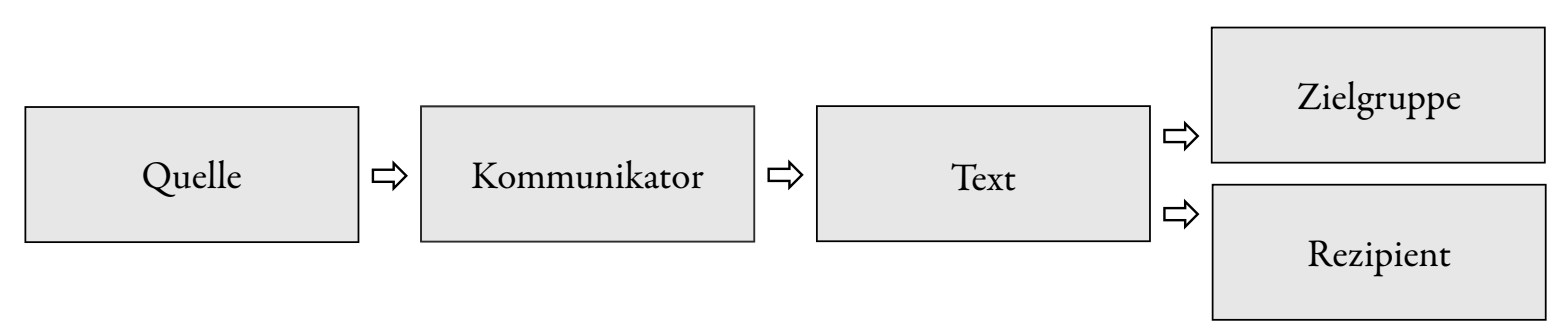

Quelle: Mayring, P. (2003), S. 50.

Das Modell lehnt sich an die Lasswell'sche Formel zur Analyse von Kommunikation („Wer sagt was, mit welchen Mitteln, zu wem, mit welcher Wirkung?“) ${ }^{181}$ an und hilft dabei, das vorliegende Datenmaterial kritisch zu spezifizieren.

Im Gegensatz zur direkten Befragung durch den Forscher selbst ist dies bei der Erhebung von Dokumenten um so wichtiger, da die Aussagekraft von nicht eigens erhobenen Schriftstücken neben der unbewussten Verzerrung auch bewusst verzerrt sein könnte. ${ }^{182}$ So ließen sich beispielsweise Imagebroschüren, welche die Vermutung des Ziels einer möglichst positiven Unternehmensdarstellung in der (ungerichteten) Öffentlichkeit zulassen, von zum internen Verbleib bestimmten Memos und Schriftstücken - teilweise mit dem Vermerk, vertraulich' gekennzeichnet - unterscheiden.

181 Vgl. Mayring, P. (2003), S. 50.

182, „...] most documents are written with a specific purpose in mind, and addressed to a specific audience." Blumberg, B. (2005), S. 194. 
Um eine möglichst hohe Pluralität der Datenquellen zu gewährleisten, wurde folglich neben der Triangulation von Dokumenten-, Archivmaterial und Interviews zusätzlich auf Divergenz von Quellen und Rezipienten innerhalb der einzelnen Datenerhebungsmethoden geachtet. Hierauf wird nun im Folgenden detailliert eingegangen.

\subsubsection{Dokumente und Archivdaten}

Dokumente geben Ergebnisse menschlichen Handelns und Denkens in schriftlicher Form wieder. ${ }^{183}$ Im Gegensatz zu den Erhebungsmethoden von Interview und Beobachtung müssen Dokumente für die Analyse nicht erst erhoben werden, sondern liegen bereits vor. Ihre Entstehung in einem natürlichen, meist forschungsfremden Hintergrund birgt somit den Vorteil, dass „nur bei der Auswahl der Dokumente, nicht aber bei der Erhebung [...] die Subjektivität des Forschers herein[spielt]. ${ }^{184}$ Es handelt sich um nicht reaktives Datenmaterial. Diesem Vorteil steht die Schwierigkeit gegenüber, dass enthaltene Informationen unvollständig oder nicht für die spezifische Forschungsfrage geeignet sein können. Als Lösung bietet sich eine verstärkte Quellenkritik an. ${ }^{185}$

Als weiterer Vorteil lässt die Analyse von Dokumenten die Untersuchung von in der Vergangenheit liegenden Phänomenen zu, welche nicht durch Beobachtung erschlossen, aber auch aufgrund eines eingeschränkten Erinnerungsvermögens Beteiligter häufig nicht mehr durch Befragung erhoben werden können. Besonders bei der Erforschung weit zurückliegender Phänomene ist die Erhebung von Dokumenten und Archivmaterial häufig die einzige Möglichkeit einer verlässlichen Datengenerierung. ${ }^{186}$

\footnotetext{
183 Vgl. Mayring, P. (1990), S. 31.

184 Mayring, P. (1990), S. 32.

185 Vgl. Mayring, P. (1990), S. 32f.

186 Vgl. Mayring, P. (1990), S. 33.
} 
Dokumente können sehr vielfältig sein. Als Beispiele lassen sich Protokolle, Briefe und Schriftverkehr, Memos, Organigramme, aber auch Zeitungsberichte und Pressemitteilungen anführen. ${ }^{187}$

Für die vorliegende Arbeit wurde eine Matrix der erhobenen Dokumente und Archivmaterialien erstellt. Auf der Abszisse ist der zu untersuchende Gegenstand - die Strategie des Bertelsmann Buchclubs sowie die Entwicklung des deutschen Buchmarktes - abgetragen. Die Ordinate unterteilt sich in Anlehnung an das in Abbildung 9 dargestellte Kommunikationsmodell nach Quelle und Rezipient. Es ergeben sich die drei forschungsrelevanten Kategorien ,interne Quelle-interner Rezipient', ,interne Quelle - externer Rezipient' sowie ,externe Quelle - externer Rezipient'. ${ }^{188}$

Das vorliegende Datenmaterial stammt aus unterschiedlichen Beständen. Während Monographien, Artikel aus der Tages- und Fachpresse sowie Veröffentlichungen des Fachverbands in Online-Archiven und öffentlichen Bibliotheken einsehbar waren bzw. auf Nachfrage direkt von der zuständigen Stelle zugeschickt wurden, sind vor allem drei Bestände gesondert hervorzuheben, da sie mit längeren Forschungsaufenthalten verbunden waren: Das Zentrale Unternehmensarchiv der Bertelsmann AG (Gütersloh), die Bestände der Bibliothek und des Historischen Archivs des Börsenvereins des Deutschen Buchhandels in der Deutschen Nationalbibliothek(Frankfurt/M.) sowie das Hanseatische Weltwirtschaftsarchiv (Hamburg).

187 Auch nicht-schriftliche Formen, wie z. B. Fotos, Bilder, Filme oder Gebrauchsgegenstände gelten als Dokumente, sofern sie den Untersuchungsgegenstand repräsentieren. Vgl. Laatz, W. (1993), S. 207.

188 ,Intern' bedeutet im Fall Bertelsmann, dass die jeweiligen Schriftstücke im Unternehmen Bertelsmann entstanden (interne Quelle) sind bzw. für den internen Verbleib bestimmt waren (interner Rezipient). Gegenteiliges gilt für die Bezeichnung, extern'. Eine Unterteilung im Fall des Buchmarktes war nicht notwendig, da sich selbst Daten für den (vertreibenden/herstellenden) Buchhandel an eine große Zielgruppe richten und somit kaum vertraulich sein können. 
Tabelle 7: Datenbasis aus Dokumenten und Archivmaterial

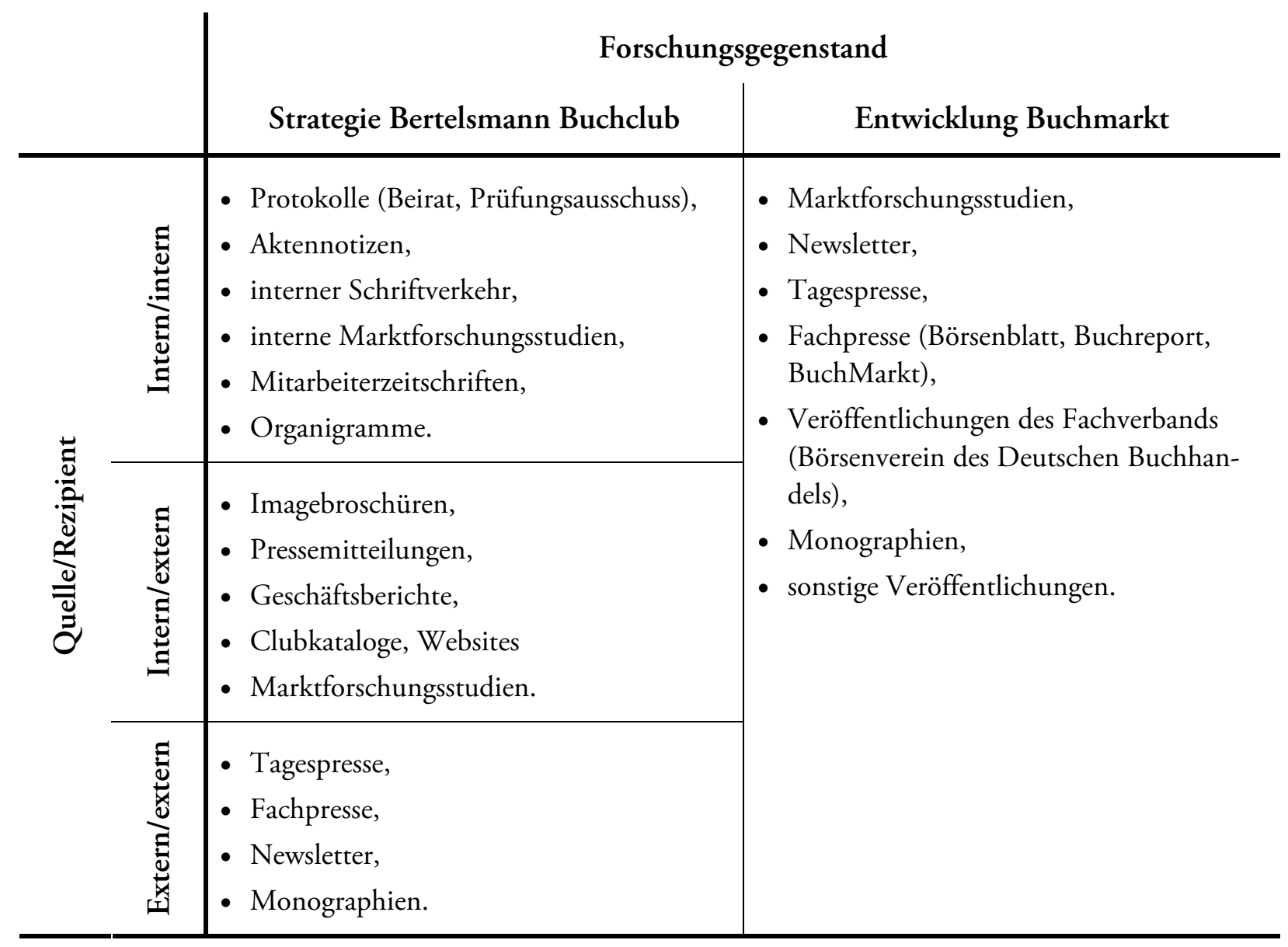

Quelle: Eigene Darstellung.

Eine Vielzahl von Dokumenten wurde in einem mehrwöchigen Rechercheprozess im Zentralen Unternehmensarchiv der Bertelsmann AG im Jahr 2006 eingesehen. Hierzu wurde ein deduktiv aus der Pfadtheorie abgeleitetes Erhebungsraster erstellt, das in einem iterativen Anpassungsprozess induktiv um zusätzliche Kategorien erweitert wurde. ${ }^{189}$ Anhand des Erhebungsrasters wurden die einzelnen im Findbuch verzeichneten Aktenbestände systematisch durchsucht und bei zutreffender Relevanz eingesehen bzw. vervielfältigt. Die Akten umfassen die Jahre von 1950 bis 2006, wobei einschränkend anzumerken ist, dass aufgrund einer dreißigjährigen Sperrfrist vor allem 
Dokumente jüngeren Datums durch die Archivleitung im Einzelfall freigegeben werden mussten. Dies betraf gleichermaßen als, vertraulich' gekennzeichnete Akten.

Ähnlich wurde im Archiv des Börsenvereins des Deutschen Buchhandels verfahren. Die Bestände der Bibliothek und des Historischen Archivs des Börsenvereins wurden in einem mehrtägigen Rechercheaufenthalt in der Deutschen Nationalbibliothek im Jahr 2006 eingesehen. ${ }^{190}$ Hierbei erfolgte unter anderem eine Vollerhebung der Buchhandels-Fachzeitschrift ,Börsenblatt des Deutschen Buchhandels' der Jahrgänge von 1950 bis 2007 . Wie schon im Unternehmensarchiv der Bertelsmann AG wurde theoriegeleitet ein nach Themen geordnetes Erhebungsraster erstellt, anhand dessen die einzelnen Jahresregister durchsucht wurden. ${ }^{191}$ Stellten sich Themenblöcke im Laufe der Recherche als unzureichend bzw. zu umfangreich heraus, wurden entsprechende Anpassungen im Erhebungsraster vorgenommen. Zusätzlich wurden zahlreiche Monographien über den Buchhandel eingesehen.

Schließlich wurde die Dokumenteneinsicht um einen mehrtätigen Forschungsaufenthalt im Hanseatischen Weltwirtschaftsarchiv in Hamburg - ebenfalls im Jahr 2006 ergänzt. Hier wurde eine Vollerhebung des Firmen-Dossiers der Bertelsmann AG, beginnend mit dem Jahr 1981, anhand eines weiteren, theoriegeleiteten Erhebungsrasters vorgenommen. ${ }^{192}$ Das Firmen-Dossier umfasst schwerpunktmäßig Pressemeldungen der nationalen sowie internationalen Tagespresse über die Bertelsmann AG. Ergänzend wurden die Geschäftsberichte der Bertelsmann AG seit ihrer Gründung 1971 bis 2005 eingesehen.

Seit 2002 befinden sich die Bestände der Bibliothek und des Historischen Archivs des Börsenvereins des Deutschen Buchhandels in der Deutschen Nationalbibliothek in Frankfurt am Main. Vgl. Deutsche Nationalbibliothek (2006).

191 Siehe hierzu Anhang 1 dieser Arbeit.

192 Siehe hierzu Anhang 1 dieser Arbeit. 


\subsubsection{Interviews}

Die zweite wichtige Quelle für die Datenerhebung in der vorliegenden Fallstudie stellt das Interview dar. In Abgrenzung zu quantitativ orientierten Umfragen sowie der großen Bandbreite unterschiedlicher Formen von qualitativen Interviews ${ }^{193}$ erfolgten die dieser Arbeit zugrunde liegenden Befragungen in Form von problemzentrierten Interviews. ${ }^{194}$ Diese zeichnen sich durch eine halb strukturierte Befragungstechnik aus, die durch einen Interviewleitfaden auf ein bestimmtes Problemfeld fokussiert ist. ${ }^{195}$ Der Interviewleitfaden gilt hierbei als grobes Interviewraster, anhand dessen die einzelnen zu erforschenden Themenfelder und Fragestellungen abgearbeitet werden können. Mit Hilfe des Leitfadens regt der Interviewer den Interviewten zu freien Narrationen an. ${ }^{196}$ Im Gegensatz zur rein narrativen Gesprächsführung, bei welcher strikt zwischen Erzähl- und Nachfragephase getrennt wird, ermöglicht das problemzentrierte Interview direkte Nachfragen und Vertiefungen der angesprochenen Inhalte. In diesem Sinne ist der Leitfaden nicht als starrer, chronologischer Ablaufplan zu verstehen, sondern Reihenfolge und Schwerpunktsetzung können im Verlauf des Interviews situativ angepasst werden. Problemzentrierte Interviews verlaufen eher dialogisch, Antworten werden nicht vorgegeben. Der Erkenntnisgewinn wird durch ein „induktiv-deduktives Wechselverhältnis“197 erreicht: Das theoretisch generierte Vorwissen wird offen gelegt und geht in Form von heuristisch-analytischen Fragestellungen dialogisch in die Interviewsituation mit ein.

193 Qualitative Interviews lassen sich grob in fünf Gruppen unterteilen: Narrative Interviews, episodische Interviews, fokussierte oder semi-strukturierte Interviews, problemzentrierte Interviews sowie ero-episodische oder ethnographische Interviews. Für eine ausführliche Gegenüberstellung siehe Helfferich, C. (2005), S. 33 sowie Hopf, C. (1991), S. 177-180; Mey, G. (2000), S. 135-151.

194 Vgl. Witzel, A. (2000).

195 Ein Beispiel für einen Interviewleitfaden findet sich im Anhang 2 dieser Arbeit.

196 Vgl. Witzel, A. (2000).

197 Witzel, A. (2000), S. 2. 
In der vorliegenden Arbeit wurde das problemzentrierte Interview eingesetzt, um Experten zu konkreten Themenschwerpunkten zu befragen. ${ }^{198}$ Auf Basis bereits vorliegender, fundierter Vermutungen wurden spezielle Experten zu den einzelnen Fragestellungen ausgewählt und systematisch befragt: „Der Experte klärt auf über ,objektive’ Tatbestände, erläutert seine Sicht der Dinge zu einem bestimmten Themenausschnitt usw. Der Experte wird hier also in erster Linie als ,Ratgeber' gesehen, als jemand, der über ein bestimmtes, dem Forscher nicht zugängliches Fachwissen ver-

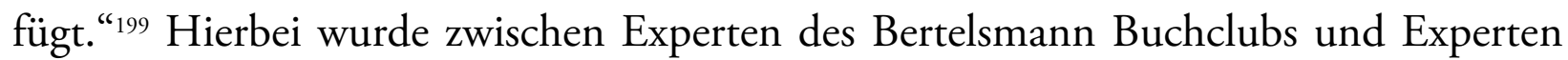
des deutschen Buchmarkts unterschieden. ${ }^{200}$ Tabelle 8 stellt die Auswahl der Personengruppen, die als Experten befragt wurden, dar. Insgesamt wurden dreizehn Experten in dreizehn Interviews befragt. Zwei Interviews wurden mit jeweils zwei Experten durchgeführt, wohingegen zwei Experten zu einem späteren Zeitpunkt ein zweites Mal befragt wurden. Die Anzahl der befragten Personen ist jeweils in Klammern angegeben. Hierbei kam es zu Überschneidungen, da beispielsweise Mitarbeiter bzw. ehemalige Mitarbeiter durch die Tätigkeit im In- und Ausland sowohl als Experten für den deutschen als auch für die ausländischen Bertelsmann Buchclubs angesehen wurden. Sechs der Interviews wurden bei den jeweiligen Interviewpartnern persönlich vor Ort geführt, die übrigen sieben telefonisch. Im Durchschnitt dauerten die Gespräche 80 Minuten, wobei das kürzeste Interview 30, das längste 180 Minuten umfasste. Elf der dreizehn Interviews wurden nach vorheriger Absprache mit den Interviewpartnern aufgezeichnet und anschließend in die schriftliche Form eines Transkriptes überführt.

198 "Der Experte verfügt über ein technisches, Prozess- und Deutungswissen, das sich auf sein spezifisches professionelles oder berufliches Handlungsfeld bezieht.” Bogner, A./Menz, W. (2005), S. 46. Zur Diskussion des Expertenbegriffs vgl. ausführlich Bogner, A./Menz, W. (2005), S. 3947 sowie Meuser, M./Nagel, U. (2005), S. 71-93. Bogner, A./Menz, W. (2005), S. 37.

200 Vor allem aufgrund des langen und weit zurückliegenden Zeithorizonts des vorliegenden Untersuchungsobjekts wurden die Interviews mit dem Bewusstsein eines potentiell eingeschränkten Erinnerungsvermögens der Auskunftspersonen geführt. Vgl. Sudman, S./Blair, E. (1998), S. $258 f$. 
Tabelle 8: Datenbasis aus problemzentrierten Experteninterviews

\begin{tabular}{|c|c|c|c|}
\hline & & \multicolumn{2}{|c|}{ Forschungsgegenstand } \\
\hline & & Strategie Bertelsmann Buchclub & Entwicklung Buchmarkt \\
\hline \multirow{3}{*}{$\frac{\mathscr{0}}{\bar{\Xi}}$} & 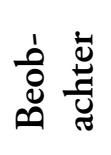 & $\begin{array}{l}\text { - Redakteure }(1 \mathrm{x}) \\
\text { - Wissenschafter }(2 \mathrm{x}) \\
\text { - Wettbewerber }(2 \mathrm{x})\end{array}$ & \multirow[t]{3}{*}{$\begin{array}{l}\text { - Redakteure (1x), } \\
\text { - Wissenschaftler }(2 \mathrm{x}) \text {, } \\
\text { - Buchhändler/Verleger }(2 \mathrm{x}) \text {. }\end{array}$} \\
\hline & 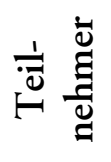 & $\begin{array}{l}\text { - Mitarbeiter (4x), davon: } \\
\text { ○ Buchclub Bertelsmann Deutschland (4x), } \\
\text { ○ Buchclubs Bertelsmann Ausland (2x). }\end{array}$ & \\
\hline & 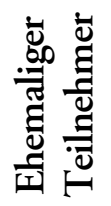 & $\begin{array}{l}\text { - Ehemalige Mitarbeiter (6x), davon: } \\
\text { o Bertelsmann AG (1x), } \\
\text { o Buchclub Bertelsmann Deutschland (5x), } \\
\text { ○ Buchclubs Bertelsmann Ausland (1x). }\end{array}$ & \\
\hline
\end{tabular}

Quelle: Eigene Darstellung.

\subsubsection{Fallstudiendatenbank und Nachvollziehbarkeit}

Zur Erhöhung der Reliabilität der Fallstudie wurde eine umfangreiche Fallstudiendatenbank angelegt, in welche sukzessive das erhobene Datenmaterial eingepflegt wurde. ${ }^{201}$ Sämtliche Daten liegen schriftlich entweder in elektronischer oder in Papierform vor. Sofern die Daten aus Archiven bereits mit einer Signatur versehen waren, wurde diese übernommen. Nicht gekennzeichnete Daten wurden mit entsprechenden Quellenangaben bzw. eigenen Signaturen versehen, um die spätere Zuordnung und Rückverfolgung lückenlos gewährleisten zu können. Interviewtranskripte umfassen die Nennung der beteiligten Personen (anonymisiert), Ort und Art des Interviews (persönlich oder telefonisch) sowie das Datum und die genaue Uhrzeit. ${ }^{202}$

Die entstandene Fallstudiendatenbank diente schließlich als Grundlage für die sich anschließende Datenauswertung. In Anlehnung an das unter Kapitel 2.5 vorgestellte zweischrittige Modell zur Pfadanalyse erfüllten die einzelnen Elemente die 
Funktion eines groben Orientierungsrasters für die Einordnung der einzelnen Daten zu den zentralen pfadtheoretischen Annahmen. Sämtliche Elemente wurden mit einer zeitlichen Dimension versehen, so dass sich Veränderungen bzw. Konstanz im Zeitablauf feststellen lassen konnten. Hierbei wurden zwei getrennte Datenbanken für das Material aus Dokumenten und Archivmaterial auf der einen und Interviews auf der anderen Seite angelegt.

Beispielhaft sei an dieser Stelle das Vorgehen anhand des Bausteins ,Umweltdynamik' als Komponente des Konstrukts, strategische Rigidität' angeführt.

Im Rahmen der Operationalisierung ergaben sich vier Kategorien zur Messung von Umweltdynamik: Wettbewerb unter den Anbietern, Substitutionsprodukte, die Verhandlungsstärke der Abnehmer sowie die politischen Rahmenbedingungen. Sofern sich anhand der erhobenen Daten weitere relevante Kategorien herauskristallisierten, wurden diese mit in die Untersuchung aufgenommen; sofern sich bestehende Kategorien als für die Forschungsfrage nicht zielführend herausstellten, wurden diese aufgegeben. In diesem Sinne verfestigte sich das endgültige Erhebungs- und Analyseraster in einem iterativen Prozess als Wechselspiel zwischen theoretischen Annahmen und empirischen Befunden.

In einem ersten Schritt wurde die Gesamtheit der auf Dokumenten und Archivmaterial beruhenden Datenquellen in das Erhebungsraster aufgenommen. Hierbei wurden relevante Textstellen zitiert, die genaue Quellenbezeichnung angegeben und mit einer eigenen Signatur versehen. Sofern es die Datenbasis zuließ, wurden vergleichbare Aussagen zu allgemeinen Themen generalisiert und schließlich zu abstrakten Kategorien reduziert. Im Ergebnis ergaben sich inhaltliche Themenschwerpunkte, beispielsweise bezüglich des Wettbewerbs im Buchmarkt, die einen Vergleich über die Zeitachse zuließen.

Selbiges Vorgehen wurde in einem zweiten Schritt mit den Daten auf Basis der geführten Interviews unternommen. Die Beispiele in Tabelle 9 aus der Kategorie, Wett- 
bewerb im Buchmarkt' auf der Grundlage von erhobenem Dokumentenmaterial verdeutlichen das methodische Vorgehen.

Einen weiteren, ergänzenden Bestandteil der Fallstudiendatenbank bilden die aus der Erhebungsmethode der teilnehmenden Beobachtung stammenden Feldnotizen. ${ }^{203}$ Im Anschluss an Interviews sowie im Rahmen der Forschungsaufenthalte in den drei genannten Archiven wurden Kommentare, erste Einschätzungen, informelle Informationen und spontane Assoziationen in schriftlicher Form festgehalten. Die Feldnotizen wurden mit entsprechenden zeitlichen, örtlichen und (anonymisierten) personenbezogenen Kennungen versehen und gingen als ergänzendes Datenmaterial mit in die Fallstudiendatenbank ein.

Auf Basis der theoretischen Grundlagen aus Kapitel 2 sowie der soeben vorgestellten Methodik sollen im nun folgenden empirischen Teil die Ergebnisse der Fallstudie des Bertelsmann Buchclubs detailliert diskutiert werden. 
Tabelle 9: Auszug aus der Fallstudiendatenbank

Beispiel 1

\begin{tabular}{|c|c|}
\hline Jahr & 1973 \\
\hline Signatur & $5 f 5-6$ \\
\hline Zitat & $\begin{array}{l}\text { „Der Buchmarkt ist schwieriger geworden. Konzerne konkurrieren mit Individualis- } \\
\text { ten. Der Konkurrenzkampf auf einem engen, sich kaum erweiternden Markt wird } \\
\text { härter.“ }\end{array}$ \\
\hline Quelle & Börsenblatt des Deutschen Buchhandels (1973, 29.06.), S. 1003. \\
\hline $\begin{array}{l}\text { Generali- } \\
\text { sierung }\end{array}$ & Ansteigender Konkurrenzkampf auf dem Buchmarkt \\
\hline Reduktion & Verdrängungswettbewerb \\
\hline \multicolumn{2}{|l|}{ Beispiel 2} \\
\hline Jahr & 1985 \\
\hline Signatur & $5 f 5-3$ \\
\hline Zitat & $\begin{array}{l}\text { „Der Gesamtmarkt Buch weist in der jüngeren Vergangenheit in den meisten Län- } \\
\text { dern keine großen Steigerungsraten auf, und auch bis zum Jahr 1990/1995 ist eher } \\
\text { mit einer mengenmäßig stagnierenden Entwicklung zu rechnen (...) Die Buchpreise } \\
\text { werden tendenziell stagnieren oder möglicherweise sinken, bei einem Trend zu ein- } \\
\text { facherer Ausstattung. Für den Buchklub-Markt gelte eine entsprechende Tendenz, } \\
\text { nämlich, 'dass Marktanteilssteigerungen der Buchclubs nur über einen verschärften } \\
\text { Verdrängungswettbewerb, nicht jedoch in einer Markterweiterung bestehen können } \\
\text { (...).“ }\end{array}$ \\
\hline Quelle & Börsenblatt des Deutschen Buchhandels (1985, 04.01.), S. 27. \\
\hline $\begin{array}{l}\text { Generali- } \\
\text { sierung }\end{array}$ & Geringes Wachstum, Verdrängungswettbewerb auf dem Buchmarkt \\
\hline Reduktion & Verdrängungswettbewerb \\
\hline
\end{tabular}

Quelle: Eigene Darstellung. 



\section{$4 \quad$ Fallstudie zum Bertelsmann Buchclub}

Die Darstellung der Fallstudie gliedert sich in vier zentrale Abschnitte. Zum genaueren Verständnis des Forschungsgegenstandes - der Strategie einer Buchgemeinschaft werden in Kapitel 4.1 zunächst grundsätzliche Informationen zum Produkt ,Buch’ sowie des relevanten Marktes, dem deutschen Buchmarkt mit dem bestehenden Buchhandelssystem, gegeben. Die Erläuterung des Buchhandelssystems wird ergänzt durch eine Diskussion zur gesetzlichen Buchpreisbindung, welche einen entscheidenden, die Strategie des Bertelsmann Buchclubs beeinflussenden Kontextfaktor bildet. Vor dem Hintergrund des deutschen Buchmarkts leitet Kapitel 4.2 zu der spezifischen Buchhandelsform der Buchgemeinschaft über, deren grundsätzliches Verständnis und historische Entwicklung ausführlich erläutert werden. Ausgehend von Buchgemeinschaften im Allgemeinen fokussiert Kapitel 4.3 auf die historischen Meilensteine des Bertelsmann Buchclubs sowie die wesentlichen strategischen Schritte der übrigen Bertelsmann-Divisionen als spezielle Kontextfaktoren. Nach der Beschreibung der unternehmenshistorischen Entwicklungen schließt sich in Kapitel 4.4 die umfangreiche Pfadanalyse zum Bertelsmann Buchclub an.

Die Pfadanalyse selbst ist wiederum in zwei wesentliche Schritte unterteilt: den Rigiditätsnachweis und die Pfaddiagnose.

\subsection{Das Buch und das deutsche Buchhandelssystem}

Der Bertelsmann Buchclub ist - wie bereits der Name verrät - eine Buchgemeinschaft und als solche auf die Herstellung bzw. den Handel mit Büchern fokussiert. Um die Arbeitsweise und die strategischen Schritte von Buchgemeinschaften im Allgemeinen und Bertelsmann im Speziellen verstehen zu können, sollen im nun folgenden Kapitel grundlegende Fakten über das Produkt ,Buch’ sowie das Marktumfeld erörtert werden. 


\subsubsection{Zum Begriff des Buches}

Das Produkt ,Buch'204 bewegt sich im Spannungsfeld zwischen Kultur und Kommerz. Auf der einen Seite transportiert ein Buch als Medium geistige Inhalte, auf der anderen Seite unterliegt es als Wirtschaftsgut den Gesetzen der Märkte, auf welchen es gehandelt wird. ${ }^{205}$ Ein Buch ist zugleich Printmedium, nichtperiodische Publikation, Kulturgut, Mittel der Wissensbereicherung und Unterhaltung sowie seinem Umfang nach ein „komplexes Bedeutungsgebilde. “206

Nach HEINOLD (2007) lassen sich neun Literaturgattungen unterscheiden: Belletristik als gehobene und reine Unterhaltungsliteratur, Kinder- und Jugendbücher, Schulbücher, Sachbücher, Fach- und wissenschaftliche Bücher, Nachschlagewerke, Bild- und Kunstbände, Kalender sowie Atlanten und Karten. ${ }^{207}$ In Abhängigkeit von der jeweiligen Literaturgattung unterscheiden sich auch die Produktmerkmale von Büchern (beispielsweise unterliegt die Absetzbarkeit von Schulbüchern starken saisonalen Schwankungen, wohingegen Sach- und Fachbücher kontinuierlich im Jahresverlauf nachgefragt werden). ${ }^{208}$ Unter Vorgriff auf das noch zu diskutierende Produktportfolio von Buchgemeinschaften sollen im Folgenden die wesentlichen Produkteigenschaften von Büchern, speziell der Gattung Belletristik, beschrieben werden. Die Schwerpunktsetzung auf Belletristik erklärt sich dadurch, dass sich vor allem in den großen Buchgemeinschaften wie dem Bertelsmann Buchclub ein Großteil des Buchangebotes aus Unterhaltungsliteratur zusammensetzt. ${ }^{209}$

Ein Buch definiert sich als „eine in Umschlag oder Einband durch Bindung zusammengefasste, meist größere Anzahl von leeren, beschriebenen oder bedruckten Blättern (mindestens 49 Seiten) von nicht periodischer Erscheinungsweise." Hiller, H./Füssel, S. (2002), S. 60 in Anlehnung an die UNESCO-Konferenz von 1964.

205 Vgl. Heinold, W.E. (2007), S. 9; Stiehl, U. (1993), S. 21f.; Meyer-Dohm, P. (1966), S. 385f.;

Faulstich, W. (1979), S. 48.

206 Stiehl, U. (1993), S. 20-34.

207 Vgl. Heinold, W.E. (2007), S. 189.

208 Vgl. Rürup, B. et al. (1997), S. 42.

209 Vgl. Kollmannsberger, M. (1995), S 132. 
Wesentliche Produkteigenschaften von belletristischen Büchern umfassen ihren Charakter als Massenware, die kalkulatorische Abhängigkeit von der Auflagenhöhe, ihre Relation zum Bildungsgrad, den Grad der Substituierbarkeit, die Eigenschaft als Prestigeobjekt sowie ihre Erklärungsbedürftigkeit. Diese Eigenschaften werden nachfolgend erläutert.

Bücher sind vorwiegend Konsumgüter, die zum privaten Gebrauch hergestellt werden. Vor allem breitenwirksame Titel der Unterhaltungsliteratur sind häufig zum Massenprodukt geworden, deren Popularität sich anhand zahlreicher Bestseller-Listen ablesen lässt. ${ }^{210}$ Die Entwicklung zum Bestseller ist jedoch keineswegs eine Selbstverständlichkeit - der Buchabsatz unterliegt starken Schwankungen. Während einige, wenige Titel nicht nur zu Best-, sondern zu Topsellern avancieren (,Harry Potter'), erweisen sich andere Titel als Flop und erreichen nur geringe Verkaufsauflagen. ${ }^{211}$ Vor allem aufgrund der noch zu diskutierenden Tatsache des hohen Fixkostenanteils der Buchherstellung erschließt sich somit zugleich die kalkulatorische Abhängigkeit des Buchhandels von der jeweiligen Auflagenhöhe. ${ }^{212}$

Bücher werden gelesen, somit ist eine zentrale Voraussetzung des Buchkonsums die Fähigkeit zum Lesen, die sich wiederum am Bildungsgrad der Bevölkerung misst. Besonders Titel der gehobenen Literatur setzen neben der generellen Lesefähigkeit zusätzlich einen bestimmten Bildungsstand voraus, damit sich dem Leser der literarische Inhalt vollständig erschließen kann. ${ }^{213}$

Lesen wiederum impliziert Lesezeit, womit das Produkt ,Buch' in unmittelbarer Konkurrenz zu weiteren Möglichkeiten der Freizeitgestaltung steht. Zentrale Substitute des Buches umfassen z. B. den Fernsehkonsum, Radio, aber auch Theater- und

210 Vgl. Heinold, W.E. (2007), S. 189.

211 Vgl. Schumann, M./Hess, T. (2005), S. 54f. Etwa 80 \% der Buchtitel bleiben in der Erstauflage. Vgl. Stiehl, U. (1993), S. 23.

212 Vgl. Schumann, M./Hess, T. (2005), S. $41 \mathrm{f}$.

213 Vgl. Rürup, B. et al. (1997), S. 39. 
Konzertbesuche, die ebenso wie das Buch zu gleichen Teilen bilden und unterhalten können. ${ }^{214}$

Der Kauf von Büchern ist durch zwei zentrale Motive geprägt. Zum einen kann die Kaufmotivation inhaltlich orientiert sein, so dass die Aufmachung, das Layout und die generelle Buchgestaltung im Vergleich zu dem eigentlichen Inhalt in den Hintergrund treten. Auf der anderen Seite werden Bücher auch als Prestigeobjekt erworben. In diesem Fall übersteigt der haptische Wert den inhaltlichen Nutzen des Buchs, da es primär dekorativen Zwecken dient und vor, nach oder anstatt dessen Lektüre im Bücherregal zur Schau gestellt wird. Die Ausstattung der Bücher ist im Fall von Prestigekäufen von besonderer Wichtigkeit. ${ }^{215}$

Schließlich lässt sich das Buch als Erfahrungsgut beschreiben, dessen subjektiver Wert sich für den Leser nicht auf den ersten Blick erschließt. Gerade angesichts der Vielzahl von jährlichen Titelproduktionen auf dem deutschen Buchmarkt ${ }^{216}$ ist der Konsument auf zusätzliche Informationen durch Produktkataloge oder in Form von Rezensionen angewiesen. ${ }^{217}$

Nach dieser grundsätzlichen Erläuterung der Produkteigenschaften von (belletristischen) Büchern, welche zu wesentlichen Teilen das noch zu spezifizierende Geschäftsmodell von Buchgemeinschaften prägen, soll im nachfolgenden Kapitel zunächst auf den relevanten Markt, den deutschen Buchhandel, eingegangen werden.

\subsubsection{Der Buchhandel als Mittler zwischen Autoren und Lesern}

Der Terminus ,Buchhandel' wird nicht einheitlich verwendet. In einer weiten Auslegung sind sämtliche an der Herstellung und Verbreitung von Büchern beteiligte Ge-

214 Vgl. Heinold, W.E. (2007), S. 53-56; Ludwig, J. (1997), S. 184.187.

215 Vgl. Rürup, B. (1997), S. 40; Rautenberg, U. (2006), S. 14-24.

216 Im Jahr 2006 erschienen auf dem deutschen Buchmarkt insgesamt 94.716 neue oder neu aufgelegte Buchtitel. Vgl. Boersenverein.de (2008a).

217 Vgl. Rürup, B. et al. (1997), S. 39-41, Sußmann, W. (1967), S. 13. 
werbezweige gemeint, wohingegen ein engeres Verständnis lediglich den Einzelhandel mit Büchern umfasst. ${ }^{218}$ Die engste Definition des Buchhandels beinhaltet ausschließlich den Sortimentshandel als dominierende Form des Bucheinzelhandels. ${ }^{219}$ Die Arbeitsdefinition in der vorliegenden Arbeit lehnt sich an das weite Begriffsverständnis an, so dass unter Buchhandel sowohl der Verlags- als auch der verbreitende bzw. vertreibende Buchhandel zu verstehen ist. Folgende Abbildung 10 verdeutlicht die einzelnen Schritte in der Mittlerfunktion des Buchhandels zwischen Autor und Leser.

Abbildung 10: $\quad$ Buchhandel zwischen Autor und Leser

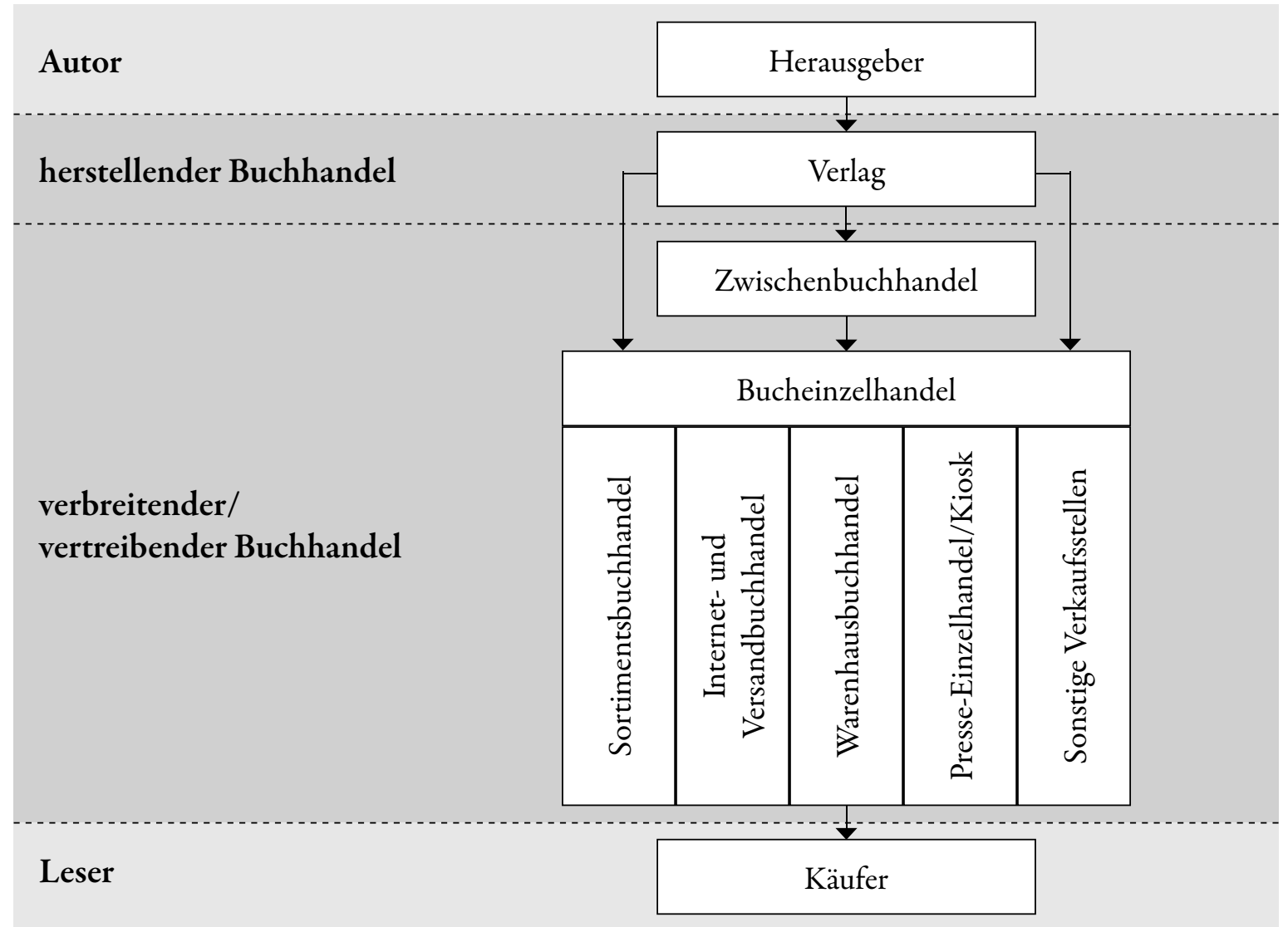

Quelle: Eigene Darstellung in Anlehnung an Heinold, W.E. (2007), S. $10 f$.

218 Vgl. Hiller, H./Füssel, S. (2002), S. 65; Stiehl, U. (1993), S. 47-49.

219 Vgl. Heinold, W.E. (2007), S. 12. 
Während mit dem herstellenden Buchhandel die mit der Finanzierung und Produktion betrauten Verlage ${ }^{220} \mathrm{zu}$ verstehen sind, ist der verbreitende Buchhandel differenzierter zu sehen. Auch wenn keine eindeutige Typologie der unterschiedlichen Vertriebsformen des Bucheinzelhandels besteht, so haben sich in Anlehnung an die jährlichen Publikationen des vom Börsenverein des Buchhandel ${ }^{221}$ herausgegebene Reihe ,Buch und Buchhandel in Zahlen' die grundlegenden Vertriebskanäle Sortimentsbuchhandel, Internet- und Versandbuchhandel, Warenhausbuchhandel, PresseEinzel-Handel bzw. Kiosk sowie sonstige Verkaufsstellen durchgesetzt. ${ }^{222}$

Mit einem Umsatz von mehr als fünf Millionen Euro pro Jahr und einem Marktanteil von 54,8 \% führt der klassische Sortimentshandel die Rangfolge der Buchvertriebswege klar an. ${ }^{223}$ Charakteristikum des Sortimentsbuchhandels ist ein vielfältiges Lager (Sortiment), aus dem Bücher, Zeitschriften, sonstige Medien oder zusätzliche Artikel gegebenenfalls durch persönliche Beratung an den Kunden verkauft werden. Nicht vorhandene Artikel können bestellt werden. Unterschieden wird weiterhin zwischen dem allgemeinen Sortiment (Fachgeschäft für Bücher), das sich durch eine breite Palette an Büchern unterschiedlicher Preis- und Qualitätsstufen auszeichnet, und dem Spezialsortiment (Spezialgeschäft), das sich beispielsweise auf bestimmte Wissenschaftsgebiete oder Genres wie Comic, Film- oder Kunstliteratur spezialisiert hat. Eine weitere Differenzierung innerhalb der Sortimentsbuchhandlung kann zwischen der

220 Ein Verlag ist „der Teil des Buchhandels, der die technische Herstellung der Bücher durch die Druckereien und Buchbindereien veranlasst.“ Hiller, H./Füssel, S. (2002), S. 330. Traditionell ist es Aufgabe der Verlage, nach Auswahl geeigneter Manuskripte, die Herstellung der Druckerzeugnisse zu veranlassen, vorzufinanzieren und für den Verkauf der fertigen Produkte zu sorgen. Vgl. Hiller, H./Füssel, S. (2002), S. 330.

221 In dem 1825 gegründeten Buchhandelsverband Börsenverein des Deutschen Buchhandels sind insgesamt ca. 6.500 Verlage, Buchhandlungen, Antiquariate, Zwischenbuchhändler und Verlagsvertreter organisiert. Vgl. Börsenverein (2007a).

222 Vgl. Heinold, W.E. (2007), S. 11.

223 Siehe hierzu die Anteile der unterschiedlichen Buchvertriebsformen im Branchen Monitor Buch, 3. Quartal 2007; Börsenverein (2007b). 
zumeist inhabergeführten Einzelbuchhandlung und Filialbuchhandlungen, die Teil eines größeren Verbunds bilden, vorgenommen werden.

Im Gegensatz zu dem stationären Sortimentsbuchhandel betreibt der Versandund Internetbuchhandel keine eigenen Ladengeschäfte, sondern wickelt seine Bestellungen via Briefpost, Telefon, Fax oder Email ab. Zum Versandbuchhandel gehört ebenfalls der Reisebuchhandel, durch dessen Vertretersystem die Verlagsangebote direkt und persönlich an die Kunden herangetragen werden.224 Mit einem Marktanteil von $11,2 \%$ stellt der Versand- und Internetbuchhandel eine bedeutende Vertriebsform der herstellenden Verlagsbuchhändler dar. ${ }^{225}$

Warenhäuser als „Einzelhandelsbetriebe, [die] in verkehrsgünstiger Geschäftslage Waren aus zahlreichen Branchen anbiete[n] “226 bilden eine weitere Form des Bucheinzelhandels. Hier werden zumeist leicht verkäufliche Titel an ein breites Publikum zu niedrigen Preisen angeboten. Nicht vorrätige Bücher können vom Kunden nicht gesondert bestellt werden. ${ }^{227}$

Der Buchverkauf über Presse-Einzelhandel wie Kioske, Pressefachgeschäfte, Supermärkte und Discounter komplettiert unter anderem mit Comics, Taschenbüchern, Romanen und Groschenheften ein meist umfangreicheres Zeitschriften- und Zeitungsprogramm. Im Unterschied zum Warenhausvertrieb ist das Buchangebot über den Presse-Einzelhandel je nach Geschäftsform lediglich als ergänzendes Randsortiment zu betrachten. ${ }^{228}$ Die Belieferung der Buchtitel erfolgt häufig über Regal-Großhändler (,Rack Jobber'). Diese beliefern und organisieren auch den Vertrieb über die sonstigen Verkaufsstellen wie Verbrauchermärkte (,Cash\&Carry'-Märkte), Tankstellen, Lebensmit-

Vgl. Rürup, B. et al. (1997), S. 59.

Vgl. Börsenverein (2007b).

Heinold, W.E. (2007), S. 39f.

Vgl. Hiller, H./Füssel, S. (2002), S. 342.

Vgl. Rürup, B. et al. (1997), S. 57. 
telfilialisten, Kaffeeröster sowie Supermarktketten wie Lidl und Aldi. Bücher bilden hier ein höchstens artverwandtes oder sogar völlig artfremdes Randsortiment. ${ }^{229}$

In Ergänzung zu den unterschiedlichen Kanälen des Bucheinzelhandels ist als tragende Säule des deutschen Buchmarktes der Zwischenbuchhandel zu nennen. Dieser besteht aus drei grundlegenden Komponenten: dem Großhandel, den Verlagskommissionären sowie den Sortimentskommissionären. ${ }^{230}$ Wesentlicher Bestandteil des Großhandels bilden die Barsortimente, die als Großhändler die Ware bei den Verlagen einkaufen und in eigenem Namen und auf eigene Rechnung an den Bucheinzelhandel weiterveräußern. Neben dieser Form des Zwischenbuchhandels im engeren Sinne besteht für die Verlage die Möglichkeit, durch Verlagskommissionäre das Lager und die Expedition der bestellten Ware von externen Dienstleistungsunternehmen, den Verlagsauslieferungen (VA), durchführen zu lassen. Auf der anderen Seite agiert der Zwischenbuchhandel durch die Sortimentskommissionäre als Dienstleister für die Buchhandlungen, indem die Großhändler als sog. Bestellanstalten die Direktbestellungen des Bucheinzelhandels an die Verlage zentral weiterleiten. ${ }^{231}$

Unter Umgehung sowohl des Zwischenbuchhandels als auch des Bucheinzelhandels erfolgt schließlich zu einem nicht geringen Anteil (16,7 \% des Umsatzes zu Endverbraucherpreisen) ${ }^{232}$ der Buchvertrieb direkt über die Verlage. Hierbei handelt es sich überwiegend um Special-Interest, Fach- und Wissenschaftsverlage, welche zumeist klar umrissene Zielgruppen bedienen. ${ }^{233}$

So vielschichtig wie die unterschiedlichen Buchvertriebsformen auftreten, so schwierig ist die vorgestellte idealtypische Differenzierung in der Praxis durchzuhalten.

Vgl. Heinold, W.E. (2007), S. 49f.

Vgl. Heinold, W.E. (2007), S. 126f.

231 Für eine ausführliche Diskussion des Zwischenbuchhandels siehe Heinold, W.E. (2007), S. 126-139.

232 Vgl. Börsenverein (2007b).

233 Ein prominentes Beispiel für den Versandbuchhandel sind periodisch erscheinende Loseblattwerke z. B. aus der medizinischen oder juristischen Fachliteratur. Vgl. Heinold, W.E. (2007), S. 36. 
Häufig vermischen sich einzelne Buchvertriebswege, so dass traditionelle Versandhäuser eigene Filialen eröffnen oder umgekehrt Filialisten den zusätzlichen Vertriebkanal des Internetversandes wählen. ${ }^{234}$ Besonders deutlich wird diese Multi-ChannelStrategie an der Vertriebsform der Buchgemeinschaft, auf die in Kapitel 4.2 intensiv eingegangen wird. Bevor Idee und Funktionsweisen von Buchgemeinschaften erläutert werden können, ist es im Vorfeld jedoch notwendig, auf ein juristisches Charakteristikum des deutschen Buchmarkts einzugehen: das Gesetz über die Preisbindung für Bücher. Nur so erschließt sich die grundsätzliche Konzeption von Buchgemeinschaften.

\subsubsection{Gesetz über die Preisbindung für Bücher}

Wie bereits in Kapitel 4.1.1 erörtert, bewegt sich das Buch im Spannungsfeld zwischen Kultur- und Wirtschaftsgut und unterliegt als solches einer gesonderten wettbewerbspolitischen Stellung. Erklärte Ziele des Gesetzgebers sind, einen leistungsfähigen Markt für Verlagserzeugnisse in Deutschland zu sichern, den Pluralismus zu schützen und die sprachliche sowie kulturelle Vielfalt zu fördern. ${ }^{235}$ In diesem Sinne soll gewährleistet werden, der Öffentlichkeit ein möglichst breites und wenig beschränktes Portfolio unterschiedlicher Bücher anbieten zu können und eine Einschränkung auf vornehmlich gut verkäufliche Bestseller-Titel zu vermeiden. ${ }^{236}$ Erreicht werden soll dies durch die gesetzliche Regelung eines einheitlichen, vom herstellenden Verlag terminierten Endverbraucherpreises für gleiche Bücher über sämtliche Vertriebskanäle. Einerseits haben so die Verlage die Chance, auf Basis einer Mischkalkulation Bestseller künstlich zu verteuern und weniger populäre Bücher preisgünstig anzubieten. Andererseits soll somit die Existenz eines breiten Buchvertriebsnetzes gefördert werden. ${ }^{237}$

234 Vgl. Heinold, W.E. (2007), S. 28.

235 O.V. (2007a), S. 1.

236 Vgl. hierzu ausführlich Franzen, H. (1987), S. 8-15 sowie Langbein, H.B. (1989), S. 13.

237 Vgl. $\$ 1$ des Buchpreisbindungsgesetzes: „Das Gesetz dient dem Schutz des Kulturgutes Buch. Die Festsetzung verbindlicher Preise beim Verkauf an Letztabnehmer sichert den Erhalt eines 
Wenn auch nicht rechtlich bindend, so wurde bereits 1887/1888 in der Satzung des Börsenvereins des Deutschen Buchhandels die buchhändlerische Preisbindung festgelegt. ${ }^{238}$ Nicht ohne kontroverse Diskussionen hat sich der feste Ladenpreis bis heute auf dem deutschen Buchmarkt etabliert, zuletzt durch das Buchpreisbindungsgesetz (BuchPrG), das zum 01. Oktober 2002 in Kraft trat. Es bestand fortan die generelle Verpflichtung für Verleger und Importeure, ihre Preise inklusive Umsatzsteuer (Endverbrauchspreise) festzusetzen. Mit Ausnahme von kurzfristig veralternden Titeln ist die Preisbindung für eine Mindestlaufzeit von 18 Monaten terminiert. ${ }^{239}$ Unter $\$ 5$, Abs.5 BuchPrG wird jedoch zugleich die gesetzliche Ausnahmeregel eingeräumt: Sofern ,sachlich gerechtfertigt', ist es zulässig, für bestimmte Titel unterschiedliche Endpreise festzulegen. Begründet wird die Einschränkung der Preisbindung wie folgt: „Absatz 5 enthält Regelungen für die Herausgabe von Parallelausgaben (wie z. B. Taschenbuchausgaben, Club-Ausgaben, Reader-Ausgaben, Jubiläumsausgaben). Danach bestimmen vor allem die Faktoren Ausstattungsunterschied, Abstand des Erscheinens oder - bei Buchgemeinschaftsausgaben - die Mitgliedschaftsbindung des Käufers über die rechtliche Zulässigkeit des Angebots eines Titels zu verschiedenen Endpreisen. “240

Durch diese Regelung wurde erstmals die Zulässigkeit von Parallelausgaben gesetzlich verankert. Wie schon in der Begründung zur Gesetzeslage konstatiert, ist dieser Zusatz vor allem für die Vertriebsform der Buchgemeinschaften relevant, auf welche im nun folgenden Kapitel detailliert eingegangen wird.

breiten Buchangebots. Das Gesetz gewährleistet zugleich, dass dieses Angebot für eine breite Öffentlichkeit zugänglich ist, indem es die Existenz einer großen Anzahl von Verkaufsstellen fördert." $\$ 1$ BuchPrG. BGB, 1. Teil I/2002, S. 3448-3450. In der Fassung vom 14.07.2006 vgl. Bramann.de (2007).

238 Die Satzungsänderung zur Buchpreisbindung von 1887/1888 war zwar nicht rechtskräftig, durch die Verbandsmacht verpflichteten sich die Mitglieder jedoch, den vom Verlag festgelegten Ladenpreis einzuhalten. Vgl. Börsenblatt (2007a).

$239 \mathrm{Vgl} . \$ 5$, Abs. 1 sowie $\$ 8$ BuchPrG in Bramann.de (2007).

240 O.V. (2007a), S. 18; WIC (2003/09). 


\subsection{Buchgemeinschaften als Mitgliedschaftssysteme}

Nach den umfassenden Erläuterungen zum Produkt ,Buch’ sowie der Beschreibung des Buchmarkts mit der gesetzlichen Buchpreisbindung sind bereits die Grundlagen zum Verständnis der Funktionsweise von Buchgemeinschaften gelegt worden. Hierauf aufbauend behandelt das nun folgende Kapitel ausführlich die zentrale Geschäftsidee von Buchgemeinschaften und geht auf wesentliche historische Entwicklungsstufen ein.

\subsubsection{Definition und Besonderheiten}

Buchgemeinschaften ${ }^{241}$ nehmen insofern eine Sonderfunktion im Buchhändlersystem ein, als dass sie weder eindeutig der Produktions-, noch der Händlerebene zuzuordnen sind. Auf der einen Seite fungieren sie als Verlag, Lizenznehmer und Lizenzgeber, auf der anderen Seite üben sie die Funktion als stationärer und ambulanter Vertriebspartner im Buchhandel aus. ${ }^{242}$ Die Buchgemeinschaft erwirbt in der Regel Lizenzrechte von Verlagen, welche die Gemeinschaft somit berechtigt, einen im Ursprungsverlag bereits erschienenen Titel für ihre Mitglieder zu vervielfältigen und zu vertreiben. Im Vergleich zum Verlag ist es den Buchgemeinschaften so möglich, die Verkaufsauflage verlässlich zu prognostizieren und das verlegerische Risiko zu verringern. Möglich ist auch der direkte Weg zwischen Autor und Buchgemeinschaft, indem diese die Originalrechte vom Autor erwirbt oder Werke direkt in Auftrag gibt. In diesem Fall kehrt sich das Verhältnis von Buchhandelsverlagen und Buchgemeinschaften um. Zur Zweitverwertung im freien Buchhandel tritt die Gemeinschaft gegenüber dem lizenznehmenden Verlagsbuchhandel als Lizenzgeber auf. ${ }^{243}$

Aufgrund der umfangreichen Historie und zahlreicher Varianten des Buchgemeinschaftsmodells fällt es schwer, eine einheitliche, umfassende Begriffsdefinition zu ge-

Die Begriffe ,Buchgemeinschaft' und „Buchklub’ sowie ,Buchclub’ werden synonym verwandt.

Vgl. Strauß, W. (1966), S. 266; Rürup, B. et al. (1997), S. 57; Heinold, W.E. (2007), S. 40;

Faulstich, W. (1974), S. 74f.; Sußmann, W. (1967), S. 38f.

Vgl. Strauß, W. (1966), S. 267. 
ben. Dennoch lassen sich einige zentrale Säulen von Buchgemeinschaften nennen, die selbige von klassischen Verlagen bzw. Bucheinzelhandelsmodellen abgrenzen.

Zentraler Gedanke von Buchgemeinschaften ist ein Abonnement, durch das die Kunden eine Mitgliedschaftsvereinbarung eingehen und sich vertraglich an die Gemeinschaft binden. Durch das System der Mitgliedschaft ist es den Buchgemeinschaften rechtlich erlaubt, das Gesetz der Buchpreisbindung zu umgehen und ihre Bücher als Nachdruckauflage in anderer Aufmachung und mit zeitlichem Abstand günstiger als im klassischen Sortimentshandel ausschließlich an ihre Mitglieder zu veräußern.

Bezüglich der Mindestdauer einer Mitgliedschaft besteht keine einheitliche Regelung, üblich sind jedoch 1- bis 2-Jahres-Perioden. Für die Dauer der Mitgliedschaft verpflichtet sich der Kunde zu dem turnusmäßigen Kauf von Waren aus dem Programmangebot der Buchgemeinschaft. Auch hier variieren die einzelnen Abnahmekonditionen mit den jeweiligen Buchgemeinschaften, und selbst innerhalb einzelner Buchgemeinschaften sind im Zeitverlauf die genauen Bedingungen zur Kaufverpflichtung sprachlich und inhaltlich häufig modifiziert worden. Üblich ist der quartalsmäßige Kauf von mindestens einem Produkt, wobei auch hier zwischen der Verpflichtung zum vierteljährlichen Buchkauf bzw. dem vierteljährlichen Kauf eines beliebigen Produkts aus dem Angebotsprogramm der Buchgemeinschaft differenziert wird. Weitere Formen der Abnahmeverpflichtung terminieren diese beispielsweise auf einen bestimmten Betrag an Mindestumsatz pro Quartal. Die jeweiligen Mitgliedschaftskonditionen ermöglichen den Buchgemeinschaften, die Kaufkraft ihrer Kunden bei der Programmzusammenstellung bereits zu verplanen und mit Mindestumsätzen zu kalkulieren.

Anders als im klassischen Sortimentshandel beschränkt sich das Spektrum des angebotenen Buchprogramms auf eine limitierte Titelauswahl, aus welcher die Mitglieder ihre Bestellungen tätigen können. Aus der starken Einschränkung der angebotenen Titel lässt sich die hohe Auflagenzahl einzelner Titel ableiten, trifft doch ein relativ hoher 
Kunden- bzw. Mitgliederstamm auf ein geringes Angebot. Das Angebotsportfolio von Buchgemeinschaften setzte sich ursprünglich ausschließlich aus Büchern zusammen, ist bei vielen Buchgemeinschaften jedoch um den sog. ,Non-Book-Bereich' erweitert worden. Der Non-Book-Bereich kann unter anderem das Angebot von CDs, DVDs, Spielen und Spielzeug, aber auch Hobby-Artikel wie Möbel und Wohnaccessoires sowie DVD-Verleih, Reisen und Versicherungen als Dienstleistungen einschließen. Unterschieden werden muss zwischen unternehmensinternen Leistungen und Leistungen, bei denen die Buchgemeinschaft lediglich als Makler auftritt, um nach Abzug von Provisionszahlungen den Auftrag in fremde Hände zu übergeben. Durch die Ergänzung des Programmangebots bietet sich für die Buchgemeinschaften vor allem die Chance, mit dem vorhandenen Mitgliederbestand Zusatzumsätze zu generieren.

Traditionell erfolgten die Kundenbestellungen über einen periodisch erscheinenden Produktkatalog, der den Mitgliedern auf postalischem Wege zugeschickt wurde. Die meisten Buchgemeinschaften haben ihre Vertriebskanäle um Filialgeschäfte erweitert, die entweder eigenständig oder in Zusammenarbeit mit dem klassischen Sortimentshandel geführt werden. Katalogbestellung und stationärer Ladenkauf sind schließlich um den Online-Vertriebsweg ergänzt worden. Die Auslieferung der Waren erfolgt in der Regel direkt, also ohne Zwischenschaltung weiterer Handelsstufen.

KOLLMANNSBERGER (1995) vereint nach eingehender Literaturanalyse die wesentlichen Eigenschaften von Buchgemeinschaften in einer sehr umfassenden Definition:

„Eine Buchgemeinschaft (Buchklub, Buchclub) ist eine Organisation, die nur ihren Mitgliedern ein Sortiment von Büchern, auch ergänzt vom Nicht-Buch-Bereich (Schallplatten, CD, Cassetten, Videos, Spiele, etc.) und Nebensortiment (Filme, Dienstleistungen, Kartenservice, Veranstaltungen etc.), in periodischer Folge per Katalog anbietet. Die Mitgliedschaft beinhaltet für das Mitglied die Abnahmeverpflichtung für ein anrechenbares Produkt pro Periode und das Abnahmerecht für beliebig viele Produkte (und Dienstleistungen). Die Mitgliedschaft läuft für eine bestimmte Zeit und wird dann verlängert oder kann gekündigt werden. Bestellung und Vertrieb erfolgen direkt oder über eigene Läden der Buchgemeinschaften oder über besondere Formen des (Sortiment)Buchhandels. Bei den Büchern handelt es sich vielfach um Lizenzausgaben, also Bücher, die im Verhältnis zur Originalausgabe mit zeitlichem Verzug in veränderter Aufmachung und zu einem unterschiedlichen Preis 
angeboten werden. Daneben können Buchgemeinschaften als Eigenverleger tätig werden. “244

Diese sehr allgemeine Begriffsbestimmung wird in Kapitel 4.3 am konkreten Fall des Bertelsmann Buchclubs spezifiziert, nachdem das nun folgende Kapitel wesentliche Schritte in der Historie von Buchgemeinschaften behandelt. Das Verständnis über die Entwicklung der Buchgemeinschaften auf dem deutschen Buchmarkt trägt vor allem dazu bei, den Blick auf die Funktionsweise der heutigen Buchclubs zu schärfen. Zusätzlich hilft die historische Betrachtung von Buchgemeinschaften, die strategische Entwicklung des Bertelsmann Buchclubs vor dem Kontext seines direkten Wettbewerbsumfelds besser einordnen und begreifen zu können.

\subsubsection{Historische Entwicklung der Buchgemeinschaften}

Die Ursprünge von Buchgemeinschaften basieren auf der Idee zur Volkserziehung und lassen sich auf das Ende des 19. Jahrhunderts zurückverfolgen - auf eine Zeit, in der das Gros der Bevölkerung wenig gebildet war und kaum eine Möglichkeit hatte, sich am kulturellen Leben der geistigen Elite zu beteiligen. ${ }^{245}$ Soziale Klassenunterschiede und der Mangel an kulturellen Gütern für breite Teile der Bevölkerung bei gleichzeitigem Drang nach Unterhaltung riefen den Gedanken der Buchgemeinschaften hervor.

So wird die Gründung des Vereins der Bücherfreunde im Jahr 1891 als offizieller Beginn der deutschen Buchgemeinschaften konstatiert. ${ }^{246}$ Geprägt von dem Geist der Zeit mit Sprüchen wie, Wissen ist Macht' und ,Das Buch dem Volke' ließ der Verein vornehmlich sog. ,Volksklassiker' drucken, um diese an seinen Abonnentenstamm zu

\footnotetext{
244 Kollmannsberger, M. (1995), S. $21 \mathrm{f}$.

245 Vgl. BUA-0041/82(1). Bereits zu Beginn des 19. Jahrhunderts existierten sog. Leseanstalten, in welchen sich ein erlesener, bibliophiler Kreis von Intellektuellen traf, um diese Foren als Möglichkeit zum geistigen Austausch zu nutzen. Da diese frühe Form von Buchgemeinschaften jedoch mit den heute vorherrschenden Ausprägungen wenig gemein haben, sei an dieser Stelle nur kurz darauf verwiesen. Vgl. hierzu die Bemerkungen in BUA-0041/82(1); Schaper, F.-W. (1977), S. 406-414.

246 Vgl. BUA-0041/82(1).
} 
verkaufen. ${ }^{247}$ Erklärtes Ziel war es, „die gesellschaftlichen Unterschiede [zu] überbrücken, die sich im Bereich des Wissens und der Bildung ebenso deutlich zeigten, wie im Wirtschaftlichen“. ${ }^{248}$ Die für die heutigen Buchgemeinschaften typischen verbilligten Lizenzausgaben sowie die Auswahlmöglichkeit für Mitglieder gab es noch nicht.

Ein weiteres wichtiges Ereignis in der deutschen Buchgemeinschaftsgeschichte ist die 1912 vom Verlag Müller errichtete Emporlese-Bibliothek, in der den Mitgliedern jährlich eine begrenzte Auswahl an Büchern angeboten wurde. Anfänglich umfasste das Programm billige Ausgaben aus der Unterhaltungsliteratur, die dann nach und nach durch anspruchvollere Werke ersetzt wurden. ${ }^{249}$ Weitere Gründungen von Buchgemeinschaften waren die Deutsche Hausbücherei im Jahr 1916, der Volksverband der Bücherfreunde 1919, der Deutschmeister-Bund im Jahr 1920 sowie die Deutsche Buchgemeinschaft im Jahre 1924. ${ }^{250}$

Bei all diesen Buchgemeinschaften stand als zentrales Leitmotiv der Volksbildungsgedanke im Vordergrund, besonders deutlich wird dies bei der 1924 gegründeten und bis heute existierenden Büchergilde Gutenberg. ${ }^{251}$ Die Büchergilde wurde gewerkschaftlich mit der zentralen Aufgabe gegründet, „neben Volksbühnenbewegung, Volkshochschule, Volksbücherei und Werksbücherei [... den] Menschen die Unentbehrlichkeit der Literatur zu lehren.“"252 Durch die Machtübernahme der Nationalsozialisten im Jahr 1933 erfuhr die Arbeit der Buchgemeinschaften einen jähen Bruch. Viele Clubs mussten ihre Arbeit einstellen, andere gingen ins Exil, wenige überlebten oder mussten ihr Programm zugunsten zur Verbreitung von Propaganda-Material ändern.

\footnotetext{
247 Vgl. Kollmannsberger, M. (1995), S. 25.

248 Strauß, W. (1974), S. 281.

249 Vgl. Gööck, R. (1968), S. 112.

250 Vgl. Gööck, R. (1968), S. 112.

251 Vgl. BUA-0041/82(1).

252 Strauß, W. (1966), S. 272.
} 
Nach dem Zweiten Weltkrieg setzten zwar einige der alten Buchgemeinschaften ihre Arbeit fort, jedoch standen die alten volksbildnerischen Ideale weniger im Vordergrund. Es vollzog sich ein „radikale[r] Umbruch[...] in Auffassung, Struktur, Arbeitsweise und Bedeutung ${ }^{{ }^{\prime 2} 23}$ der Buchgemeinschaften:

Um die Nachfrage im Buchhandel wiederzubeleben, suchten Verleger und Buchhändler nach neuen Absatzwegen. Geleitet wurden sie von der Idee, „Menschen für das Buch und den Buchhandel zu gewinnen, die bisher glaubten, ohne beides existie-

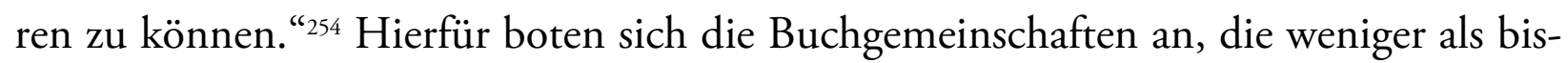
her dem Anspruch zur Volkserziehung als vielmehr der Idee einer effektiven Vertriebsform genüge tun sollten. So erfolgte 1947 die Neugründung der Büchergilde Gutenberg, 1948 entstand der Christliche Bildungskreis, und 1950 wurden gleich drei Buchgemeinschaften gegründet: Der Europäische Buchclub, die Buchgemeinschaft Bücher für alle sowie der Lesering von Bertelsmann. 1952 starteten der Fackel-Buchklub und die Herder-Buchgemeinde. Im selben Jahr existierten bereits 38 Buchgemeinschaften mit zusammen mehr als einer Million Mitgliedern auf dem deutschen Buchmarkt. ${ }^{255}$

Zwischen den rivalisierenden Unternehmen entwickelte sich ein harter Konkurrenzkampf um die Werbung neuer Mitglieder. Sukzessive setzte sich der Bertelsmann Lesering am Markt durch. Er übernahm von 1952 an fast 20 kleinere und mittelgroße Konkurrenzclubs sowie die zweitgrößte Gemeinschaft, die Europäische Bildungsgemeinschaft, die 1981 ca. 1,6 Millionen Mitglieder zählte. Dazu beteiligte sich Bertelsmann noch zu 50\% an der Deutschen Buch-Gemeinschaft und kaufte sie 1988 völlig auf. Ende 1960 war der Buchgemeinschaftsmarkt auf etwa 15 Buchclubs geschrumpft, die insgesamt rund fünf Millionen Mitglieder zählten. ${ }^{256}$

Strauß, W. (1966), S. 273.

254 Hiller/Strauß (1975): Buchhandel, S. 284.

255 Vgl. Kollmannsberger, M. (1995), S. 30.

256 Vgl. BUA-0006/129. 
Der Markt wurde von zwei großen Blöcken, Bertelsmann und Holtzbrinck, beherrscht. ${ }^{257}$ Schließlich führt Bertelsmann seit der Übernahme des Deutschen Bücherbundes von Holtzbrinck im Jahr 1992 den Sektor der Buchgemeinschaften mit einem Marktanteil von $97,5 \%$ unangefochten an. ${ }^{258}$

Zusammenfassend lässt sich festhalten, dass die traditionsreiche Idee der Buchgemeinschaft vor allem nach dem Zweiten Weltkrieg als gewinnorientierte Verkaufsund Vertriebsstrategie eingesetzt wurde. Aus der Vielzahl von Buchclubgründungen der 50er Jahre setzte sich sukzessive der Bertelsmann Buchclub durch, übernahm fast sämtliche Wettbewerber und beherrscht den Markt der Buchgemeinschaften heute nahezu monopolistisch. Diese Entwicklung des Bertelmann Buchclubs soll im nachfolgenden Kapitel 4.3 aufgegriffen und in Vorbereitung auf die anschließende Pfadanalyse vertieft werden.

\subsection{Der Bertelsmann Buchclub und die Bertelsmann AG: Historische Entwicklung und Meilensteine}

Die Betrachtung der historischen Meilensteine des Bertelsmann Buchclubs kann nicht isoliert von der Konzernstruktur der heutigen Bertelsmann AG geschehen, basiert gerade ein Großteil der heutigen Konzerndivisionen von Bertelsmann auf den strategischen Entwicklungsstufen des Buchclubs. Aus diesem Grund erfolgt im Anschluss an die Darstellung der Buchclubhistorie eine Betrachtung der übrigen Konzerndivisionen, anhand derer sich die zentralen Kontextfaktoren für die nachfolgende Pfadanalyse des Bertelsmann Buchclubs ergeben. Ziel der umfangreichen Betrachtung der einzelnen Konzerndivisionen ist es somit nicht, eine rein historische Aufarbeitung zu leisten, sondern den Hintergrund relevanter, den Buchclub beeinflussender Kontextfaktoren zu ermitteln.

257 Vgl. Rühle, G. (1985), S. 42-50.

258 Vgl. Kollmannsberger, M. (1995), S. 46. 
Einschränkend ist vorwegzunehmen, dass im Laufe der über 57-jährigen Unternehmensgeschichte zahlreiche Restrukturierungsprozesse, die nicht selten mit $\mathrm{Na}$ mens- und Rechtsformänderungen einhergingen, vorgenommen wurden. In der vorliegenden Arbeit wurde versucht, wesentliche Änderungen zu erfassen. Aufgrund der Vielzahl von Modifikationen und organisatorischen Verzweigungen sowie des langen Zeithorizonts wurde jedoch sowohl aus forschungspragmatischen als auch aus Gründen der Übersichtlichkeit auf eine vollständige Darstellung verzichtet.

\subsubsection{Vom Lesering zur Direct Group}

Der deutsche Bertelsmann Buchclub blickt auf eine lange (Verlags-)Geschichte zurück. Um für die Pfadanalyse relevante Einflussfaktoren identifizieren zu können, ist es notwendig, zunächst den Blick auf die Ursprünge, den C. Bertelsmann Verlag, zu richten. Die Tatsache, dass das Unternehmen Bertelsmann bereits vor der Buchclubgründung im Jahr 1950 existierte, lässt die Vermutung zu, dass strategische Entscheidungen schon vor 1950 auf Gründungs- und Anschlussentscheidungen im späteren Buchclub ausstrahlten. Das folgende Kapitel behandelt daher die wesentlichen Entwicklungsstufen dieser frühen Verlagsgeschichte, um im darauf aufbauenden, anschließenden Kapitel detailliert auf die zentralen Meilensteine der daraus resultierenden Buchgemeinschaft von Bertelsmann einzugehen. Ergänzend findet sich im Anhang 3 eine detaillierte Übersicht über die wesentlichen strategischen Entwicklungsstufen von 1950 bis 2007.

\subsubsection{Der C. Bertelsmann-Verlag (1835-1950)}

Der eigentliche Ursprung der heutigen Bertelsmann AG lässt sich auf das Jahr 1835 zurückdatieren - das Jahr, in welchem der gelernte Buchbinder Carl Bertelsmann in Gütersloh die Verlagsbuchhandlung ,C. Bertelsmann’ gründete. ${ }^{259}$ Zum Verlagspro- 
gramm gehörten vor allem Lieder- und Notenbücher, kirchliche Schriften sowie Schulbücher; vorübergehend wurde auch eine Heimatzeitung verlegt. Im Jahr 1850 trat Carl Bertelsmanns Sohn Heinrich die Verlagsnachfolge an und baute das Unternehmen in den anschließenden Jahren von 14 auf 60 Mitarbeiter aus. Das bisherige Programm wurde um Werke der wissenschaftlichen Theologie, Philologie und Geschichte erweitert. Schließlich übernahm Heinrich Bertelsmanns Schwiegersohn Johannes Mohn 1887 die Verlagsleitung, Dieser setzte den verlegerischen Schwerpunkt auf Missions- und kirchliche Literatur sowie Schul- und Jugendliteratur. ${ }^{260}$ In vierter Familiengeneration leitete ab dem Jahr 1921 Johannes Mohns Sohn Heinrich den Gütersloher Verlag. Unter Heinrich Mohns Führung erfolgte 1928 erstmals eine Ausdehnung des christlich und theologisch geprägten Verlagsprogramms auf schöngeistige Werke. ${ }^{261}$ Es wurden sog. ,Volksausgaben' - verbilligte Sonderausgaben volkstümlicher Romane - mit großem Erfolg in hohen Auflagenzahlen verlegt und in Zusammenarbeit mit dem vertreibenden Buchhandel erstmals Werbemaßnahmen durchgeführt. ${ }^{262}$ Binnen weniger Jahre verlagerte sich der literarische Schwerpunkt des mittlerweile auf 400 Mitarbeiter angewachsenen Verlagshauses hin zu schöngeistigen Werken. Aufgrund von Rohstoffknappheit konnte die Herstellung der Volksausgaben während des Zweiten Weltkriegs nicht fortgesetzt werden, jedoch erzielte die Herausgabe von sog. ,Feldpostausgaben' ebenfalls eine große Breitenwirkung. ${ }^{263}$ In den letzten Kriegsjahren erfolgte dann ein tiefer Einschnitt in der Geschichte des Bertelsmann Verlags. Das nationalsozialistische Regime erwirkte 1944 die Verlagsschließung, leitende Mitarbei-

260 Vgl. BUA-UP3/26-7 (Bertelsmann Prospekt, 1959), S. 3.

261 Schöngeistige Literatur wird auch als Unterhaltungsliteratur bezeichnet und entspricht in etwa der heutigen Bezeichnung ,Belletristik'. Vgl. Hiller, H./Füssel, S. (2002), S. 42; 281.

262 Vgl. Gööck, R. (1968), S. 46-54; FAZ (1985, 15.06.).

263 ,Feldpostausgaben' waren kleine Hefte mit vornehmlich deutschen Erzählungen, die dazu dienten, die deutschen Truppen an der Front mit Literatur zu versorgen. Vgl. Tietz, B. (1985), S. 340. 
ter wurden verhaftet und große Teile des Betriebsgebäudes - mit allen dort lagernden Material- und Buchvorräten - durch einen Bombenangriff im Jahre 1945 zerstört. ${ }^{264}$

Nach fast zwei Jahren des Wiederaufbaus wurde die Verlagsarbeit des C. Bertelsmann Verlags im Jahr 1946 fortgesetzt. Ein Jahr später übernahm Heinrich Mohns Sohn Reinhard die Verlagsleitung, seine beiden Brüder Sigbert und Gerd folgten kurze Zeit später mit der Wahrnehmung verlegerischer Führungspositionen. Warenmangel und Geldüberfluss in den ersten Nachkriegsjahren bedingten einen florierenden Buchmarkt und stark steigende Umsätze für den Bertelsmann Verlag. Die hohe Buchnachfrage wurde jedoch bereits im Jahr 1949 durch die „Buchkrise’ abgelöst. Das Warenangebot in den Geschäften nahm zu, und die Bevölkerung - bedingt durch die Währungsreform 1948 - befriedigte zunächst ihre Nachfrage nach grundlegenden Gütern wie Möbeln, Textilien und Nahrungsmitteln. Sowohl kulturelle als auch unterhaltende Angebote wie Schauspiel, Musik und Bücher wurden sukzessive zu Luxusgütern. ${ }^{265}$ Hinzu kam, dass gerade das vom Bertelsmann Verlag forcierte unerhaltende Buch in zunehmende Konkurrenz zu Kino, Rundfunk und Illustrierten geriet, so dass sich die Frage nach einem grundsätzlich abnehmenden Lesebedürfnis stellte. Die Aufgabenstellung des Buchhandels wurde von Bertelsmann neu definiert:

„Die Behauptung des Buches hängt weitgehend davon ab, ob und wie es dem Publikum, das im Bereich des materiellen Verbrauches und der publizistischen Massenmedien zur Konsumhaltung angeregt und erzogen wird, angeboten wird. Der Typ des Buchhändlers im überkommenen Stil hat sich an den Bedürfnissen der 4 bis $5 \%$ Gebildeten entwickelt. Selbst die Beschränkung auf diesen Kreis bzw. diese Lese-Interessen bedeutet angesichts der jährlichen Vervielfachung anspruchsvoller Literatur eine erhebliche Belastung oder gar Überforderung. Will er aber auch die anderen Leserkreise bzw. Literaturinteressen seiner bisherigen Kunden befriedigen, muss er nach neuen Formen suchen, die sich an den Gesetzen der modernen Werbung orientieren. ${ }^{" 266}$

264 Vgl. Adron, L. (1983).

265 Vgl. Gööck, R. (1968), S. 102-104.

266 BUA-0041/2 (Lesering: Entwicklung und Aufgabe, 1961), S. 7, in Anlehnung an ein Zitat des Soziologen Franz Ronneberger. 
Unterschiedliche Wege zur Belebung des Verlagsgeschäfts wurden diskutiert, so z. B. die Herausgabe moderner und preiswerter Taschenbücher, die Gründung einer Buchgemeinschaft sowie die Herausgabe von saisonal unabhängigen Fachbüchern. ${ }^{267}$ Schließlich wurde am 01.07.1950 die Buchgemeinschaft, Lesering Das BertelsmannBuch' gegründet. ${ }^{268}$

\subsubsection{Gründung und Aufbau (1950-1970)}

Die ersten zwanzig Jahre nach der Buchclubgründung waren gekennzeichnet von expansiver Aufbauarbeit, der Ausweitung der Werbe- und Vertriebskraft sowie der sukzessiven Erweiterung eigener Produktionskapazitäten.

Wie in Kapitel 4.2.2 bereits festgestellt, war die Idee einer Buchgemeinschaft nicht neu. Innovativ war allerdings die von Bertelsmann propagierte aktive Einbindung der Infrastruktur des Buchhandels durch die sog. Zweistufigkeit des Leserings:269 „Es [konnte] niemand beim Verlag, aber jeder bei jeder Buchhandlung Mitglied im Bertelsmann Lesering werden. “270 In diesem Sinne wurden großteilige Aufgaben der Mitgliederverwaltung an Firmen des Buchhandels, die sog. Vertriebsfirmen, ausgelagert. Diese Vertriebsfirmen waren - und sind es z. T. noch heute - rechtliche Eigentümer der Mitgliederkarteien. Konkret bedeutete dies, dass die Vertriebsfirmen Mitgliedskonten führten, den Katalog versendeten, bestellte Titel auslieferten und Reklamationen bearbeiteten. Bertelsmann hingegen war für die Programm- und Kataloggestaltung, Beschaffung, Lagerhaltung sowie die Auslieferungen an die Vertriebsfirmen ver-

267 Vgl. Schuler, T. (2005), S. $158 f$.

268 Für die genaueren Umstände der Leseringsgründung siehe ausführlich BUA-0041/2 (Lesering: Entwicklung und Aufgabe, 1961), S. 8-15; BUA-UP3/26-7 (Bertelsmann Prospekt, 1959), S. 1621; Gööck, R. (1968), S. 110-121; Tietz, B. (1985), S. 341-346; sowie der Rundbrief mit der Verkündigung der Leseringsgründung an den Buchhandel BUA-0031/1 (Lesering: Gründung, 1950).

269 Für eine detaillierte Gegenüberstellung der einstufigen und zweistufigen Konzeption von Buchgemeinschaften vgl. Schaper, F.-W. (1977), S. 439-445.

270 Gööck, R. (1968), S. 115. 
antwortlich. Als Ausgleich für die übernommenen Tätigkeiten erhielten die Vertriebsfirmen definierte Provisionszahlungen für die Umsätze ihrer Mitglieder. ${ }^{271}$

Zu großen Teilen erfolgte diese Zweistufigkeit in Kooperation mit dem Reise- und Versandbuchhandel, ab 1952 wurde die aktive Mitgliederwerbung und -betreuung zusätzlich vom Werbenden Buch- und Zeitschriftenhandel (WBZ) übernommen. ${ }^{272}$ Auch der klassische Sortimentshandel wurde mit Werbungs- und Betreuungsleistungen der Mitglieder einbezogen, so dass zum einen eine weitgehend friedliche Koexistenz von Sortiment und Buchclub gewährleistet wurde und zum anderen die bereits bestehende Werbe- und Vertriebsstruktur des vertreibenden Buchhandels zur exponentiellen Ausweitung des Mitgliederbestands genutzt werden konnte. ${ }^{273}$ Erfolgte die Mitgliederwerbung anfänglich durch eigene oder fremde Verlagsvertreter, so wurde im Jahr 1951 das System der Freundschaftswerbung eingeführt: Mitglieder werben Mitglieder und erhalten als Prämie einen kostenlosen Buchtitel. ${ }^{274}$

Auch diese Werbestrategie beschleunigte den ohnehin stark anwachsenden Mitgliederbestand erneut, so dass im Jahr 1954 mit der Gründung der Verlagsgemeinschaft Bertelsmann Lesering GmbH in Rheda (VG Rheda), in der die Mitgliederverwaltung und -betreuung der einzelnen Vertriebsfirmen zusammengefasst wurden, ${ }^{275}$ erste organisatorische Anpassungen erfolgten.

271 Vgl. Schaper, F.-W. (1977), S. 441f.; Tietz, B. (1985), S. 342; Beinsen-Ruf, H.-L. (1980), S. 16.

272 Der Werbende Buch- und Zeitschriftenhandel sorgte traditionell für die Anwerbung und Belieferung von Buch- und Zeitschriftenabonnenten. Vgl. Gööck, R. (1968), S. 130 sowie BUA0041/2 (Lesering: Entwicklung und Aufgabe, 1961, S. 11-14.

273 Vgl. Tietz, B. (1985), S. 342; Kollmannsberger, M. (1995), S. 31. Eine Ausnahme dieses Systems der Mitgliederbetreuung durch fremde Vertriebsfirmen bildete die 1951 von Bertelsmann gegründete hauseigene Versandbuchhandlung Schloss-Verlag, welche die nicht von externen Vertriebsfirmen geworbenen Mitglieder verwaltete. Vgl. Tietz, B. (1985), S. 346.

274 Vgl. Gööck, R. (1968).

275 Vgl. WIC (1994/06), S. 3. Der nach Abzug der Geschäftskosten verbleibende Gewinn der VG Rheda wird an die Vertriebsfirmen in voller Höhe als Rendite / Pacht ausgezahlt, so dass die VG Rheda selbst keinen Gewinn machen darf. Vgl. Schaper, F.-W. (1977), S. 444. 
Neben der Zentralisierung der Mitgliederverwaltung wurde im selben Jahr der Druckereibetrieb unter der Firmenbezeichnung, Mohn \& Co. GmbH' grundlegend erweitert, da die eigenen Produktionskapazitäten zur Belieferung des ansteigenden Buchbedarfs im Leserings nicht mehr ausreichten. ${ }^{276}$ Die expansive Ausweitung von Mohn \& Co. mündete schließlich erstmals 1955 in einer organisatorischen Trennung der Leseringarbeit von Verlag und Technik unter der Firmenbezeichnung Bertelsmann Lesering GmbH. ${ }^{277}$ Im darauf folgenden Jahr 1956 erfolgte neben der Gründung der eigenen Werbe- und Vertriebsorganisation Vertriebsgemeinschaft Buch und Wissen ${ }^{278}$ als weiterer Meilenstein die Gründung des Bertelsmann Schallplattenrings. ${ }^{279}$ Der Schallplattenring arbeitete nach den gleichen Geschäftsprinzipien wie auch der Lesering (und der im Jahr zuvor gegründete Jugend-Lesering), ${ }^{280}$ konnte im Gegensatz zu diesen jedoch weder auf hauseigene Lizenzen noch auf Produktionsstätten zurückgreifen.

In Reaktion auf die Schwierigkeiten beim Lizenzerwerb für Musiktitel erfolgte eine erneute Ausweitung hauseigener Produktionskapazitäten: Im Jahr 1958 gründete Bertelsmann auf technischer Seite die eigene Schallplattenproduktionsfirma Sonopress GmbH sowie auf inhaltlicher Seite - zum Aufbau eines eigenen Repertoires - die Produktionsfirma und Tochtergesellschaft von Mohn \&Co. Ariola Schallplatten

Vgl. Gööck, R. (1968), S. 154.

Vgl. Gööck, R. (1968), S. 160f.

Vgl. hierzu ausführlich BUA-UP3/26-7 (Bertelsmann Prospekt, 1959), S. 31-33.

Vgl. BI (1960/09). Mit der Gründung des Schallplattenrings wurde die Bertelsmann Lesering GmbH in Bertelsmann GmbH umbenannt. Vgl. Gööck, R. (1968), S. 160.

280 Der 1955 gegründete Bertelsmann Jugend-Leserings legte eine eigene Programmillustrierte auf und erhob niedrigere Mitgliedsbeiträge als der klassische Lesering unter sonst gleichen Clubbedingungen. Der Jugend-Lesering wurde zum 01.07.1965 in den Bertelsmann-Lesering integriert und deren Mitglieder in den Mitgliederbestand des Leserings überführt. Vgl. BUA-0006/15 (Bertelsmann-Clubarbeit: Chronik, 1977), S. 56; BUA-UP2/128-1955 (Lesering-Rundbrief, 1955); Gööck, R. (1968), S. 156f.

Zusätzlich wurde im Jahr 1961 der Europaring der Buch-und Schallplattenfreunde als eine Kombination von Lese- und Schallplattenring gegründet. 
GmbH. ${ }^{281}$ Aufgrund des expansiven Wachstums erfolgten 1959 weitere Restrukturierungsprozesse bei Bertelsmann. Auf Verlagsseite wurden die bestehenden Einzelfirmen neu gegliedert, ${ }^{282}$ und die neu gegründete Firma Kommissionshaus Ton und Buch zentralisierte Lagerhaltung, Rechnungswesen und Versand. ${ }^{283}$

Neben der expansiven Werbe- und Vertriebsstrategie, der Ausweitung von Produktionskapazitäten und organisatorischen Restrukturierungen lässt sich als weiterer strategischer Meilenstein die Erweiterung der Vertriebswege benennen. Erfolgte das Buchclubgeschäft bislang ausschließlich über einen Bücherkatalog auf dem Versandweg, ${ }^{284}$ so wurde im Jahr 1964 das erste stationäre Ladengeschäft unter dem Namen ,Bücherstube' in Kiel eröffnet. ${ }^{285}$ Die Filialisierung wurde in den darauf folgenden Jahren weiter forciert und großflächig ausgebaut. Zusätzlich eröffnete 1966 die erste Partner-Club-Filiale. In Kooperation mit dem klassischen Sortimentshandel wurden Club-Verkaufsflächen in bestehende Bucheinzelhandelsgeschäfte integriert. Auch dieses Konzept wurde sukzessive ausgebaut. ${ }^{286}$

Weitere wesentliche Entwicklungen der 60er Jahre waren die beginnenden Übernahmen von Wettbewerbern, Clubneugründungen sowie der Start einer Expansionswelle ins Ausland. 1963 wurden der Europäische Buch- und Phonoclub (beide wurden später in Europäische Bildungsgemeinschaft umbenannt) ${ }^{287}$ als auch der Spezialclub für klassische Musik Ring der Musikfreunde (P.P. Kelen) übernommen. ${ }^{288} 1965$ erfolgte

Vgl. Gööck, R. (1968), S. 179; Tietz, B. (1985), S. 351; BUA-006/465 (Hennig, 1977); BUAUP3/26-7 (Bertelsmann Prospekt, 1959), S. 29.

Vgl. hierzu ausführlich Kapitel 4.3.2.2 dieser Arbeit.

Vgl. Gööck, R. (1968), S. 188.

Anfänglich diente der Verlagsprospekt von C. Bertelsmann unter dem Namen ,Bertelsmann Illustrierte' als Bücherkatalog, aus welchem die Mitglieder unter Umgehung des stationären Bucheinzelhandels direkt ihre Bestellung treffen konnten. Vgl. Gööck (1968), S. 117.

Vgl. Gööck, R. (1968), S. 216; Adron, L. (1983). Die Umbenennung von ,Bücherstube’ zu ,Club-Center' erfolgte im Jahr 1969. Vgl. BuchMarkt (1978/11), S. 42.

286 Vgl. WIC (2003/April), S. 4.

287 Vgl. Gööck, R. (1968), S. $211 \mathrm{f}$.

288 Vgl. WIC (1987/06-07), S. 1f. 
die Gründung des Christlichen Bildungskreises - eine Buchgemeinschaft mit vornehmlich christlicher Literatur, ${ }^{289}$ und schließlich zählte ab 1969 auch die Buchgemeinschaft Deutscher Bildungskreis zu den Buchclubs von Bertelsmann.

Parallel zu den Erweiterungen auf dem deutschen Buchmarkt erfolgte ab 1962 die konsequente Ausweitung der Buchclubidee in ausländische Märkte. Den Anfang machte die Gründung des Circulo de Lectores in Spanien, woraufhin immer mehr Neugründungen oder Beteiligungen an bereits bestehenden ausländischen Verlagen und Buchgemeinschaften unternommen wurden. Nach der Expansion in die Schweiz und Österreich im Jahr 1962 wurde 1967 die Clubarbeit durch die Gründung des Europaclub International voor Boeken en Platen in den Niederlanden und Belgien aufgenommen; 1969 erfolgte die Expansion nach Mittel- und Südamerika.

\subsubsection{3 Übernahmen und Auslandsexpansion (1970-1990)}

Die Tendenzen der 60er Jahre verstärkten sich in den darauf folgenden Jahren weiter. Vor allem die Übernahme von konkurrierenden Buchgemeinschaften in Deutschland sowie die weitere Expansion in ausländische Märkte prägten zu großen Teilen die Phase von 1970 bis 1990.

Nachdem die natürliche Wachstumsphase weitestgehend abgeschlossen war und sich bereits Marktsättigungstendenzen bemerkbar machten, ${ }^{290}$ erweiterte Bertelsmann trotz nur verhaltenem Gesamtmarktwachstum seinen Marktanteil radikal von $17 \%$ im Jahr 1970 bis Mitte der 80er Jahre auf $66 \%{ }^{291}$ Möglich war diese Entwicklung durch einen zunehmenden Konzentrationsprozess. Die Zunahme des Marktanteils ist neben der Übernahme zahlreicher kleinerer Buchgemeinschaften vor allem auf die 50 \%-Beteiligung an der Deutschen Buchgemeinschaft im Jahr 1970 sowie die vollständige Übernahme der Europäischen Bildungsgemeinschaft im Jahr 1973 zurückzufüh-

\footnotetext{
289 Vgl. Gööck, R. (1968), S. $211 \mathrm{f}$.

290 Vgl. BG 1977/78, S. 12.

291 Vgl. Roszinsky-Terjung, A. (1981), S. $113 f$.
} 
ren. ${ }^{292} \mathrm{Da}$ beide Buchgemeinschaften auch eigene Filialgeschäfte unterhielten, bedeutete deren Akquisition für Bertelsmann zugleich auch die Übernahme von weiteren Filialketten, die zunächst als eigenständige Buchclubketten weitergeführt wurden. ${ }^{293}$ 1989 kaufte Bertelsmann die verbleibenden Anteile an der Deutschen Buchgemeinschaft vollständig auf. ${ }^{294}$ Ergänzend ist anzuführen, dass die letzte große Übernahme 1991 zunächst durch eine 24,9\%-Beteiligung an der zum Holtzbrinck-Verlag gehörenden Buchgemeinschaft Deutscher Bücherbund erfolgte, dessen Anteile nur ein Jahr später vollständig erworben wurden, so dass Bertelsmann seinen Marktanteil im Jahr 1992 auf $97,5 \%$ ausweiten konnte. ${ }^{295}$

Übernahmen und Neugründungen zogen schließlich auch organisatorische Konsequenzen nach sich. Im Jahr 1971 wurde die Bertelsmann $A G$ gegründet, in welcher die ,Buch- und Schallplattengemeinschaften’ neben den Divisionen ,Musik, Film, Fernsehen’, ,Verlage' und ,Technik' als eigene Sparte geführt wurden. ${ }^{296}$ Innerhalb dieser Sparte wurden 1978 sämtliche Buch- und Musikgemeinschaftsfirmen in die Dachmarke Bertelsmann-Club überführt. Hierzu zählten bis zu diesem Zeitpunkt die Einzelfirmen Bertelsmann Reinhard Mohn GmbH (mit Lese-, Schallplatten- und Europaring sowie dem Deutschen Bildungskreis), die Europäische Bildungsgemeinschaft sowie die Deutsche Buchgemeinschaft. ${ }^{297}$ Die Zusammenführung der einzelnen Buchclubs

292 Vgl. BUA-0006/464 (Deutsche Buchgemeinschaft, 1970); Vgl. BG (1977/78), S. 13.

293 Vgl. Lehning, T. (2004), S. 58.

294 Vgl. WIC (1989/04), S. 5.

295 Vgl. WIC (1992/06), S. 2. Analog zur Entwicklung der Bertelsmann Buchgemeinschaften wurde bereits seit Mitte der 60er Jahre in weitere Märkte (z.B. den Film- und Zeitschriftenmarkt) diversifiziert. Da diese bis heute bestehenden Unternehmenssparten nicht zum fokalen System der vorgenommenen Analyse zählen, jedoch als Umsysteme durchaus relevant sind, werden die parallelen Unternehmensentwicklungen auf Konzernebene in Kapitel 4.3.2 gesondert dargestellt.

296 Vgl. BG (1973/74), S. 13.

297 Vgl. BG (1977/78), S. 13. 
im Jahr 1978 resultierte zusätzlich in dem nun mehr einheitlichen Auftritt der mehr als 270 Club-Center. ${ }^{298}$

Neben der Übernahme der zentralen Wettbewerber sowie internen Reorganisationsprozessen reagierte Bertelsmann nur verhalten auf die inländischen Sättigungserscheinungen im Markt der Buchgemeinschaften. Wesentliche strategische Schritte umfassten die Erweiterung des Angebotsprogramms um Spiele (1972), Kameras (1974), kunstgewerbliche Erzeugnisse (1975), Fotolabor-Services (1975) sowie weitere Freizeitartikel (1976). ${ }^{299}$ Vor allem in den 80er Jahren erfolgten zusätzlich die Modernisierung der Clubfilialen, eine offensivere Kundenansprache sowie die Verbesserung der Werbemittel. ${ }^{300}$

Blieben radikale strategische Neuerungen im Inland weitestgehend aus, so wurde die bereits in den 60er Jahren begonnene Expansionsstrategie ins Ausland auch in den 70er und 80er Jahren offensiv weiterverfolgt. 1970 wurde - zunächst durch eine 50\%Beteiligung - der französische Buchclub France Loisirs gegründet. Der Club Pladeringen wurde 1973 in Dänemark, der Euroclub 1974 in Italien und der Leisure Circle 1977 in Großbritannien eröffnet. Schließlich trat Bertelsmann durch die Übernahme der Doubleday-Buchclubs in den USA, England, Kanada, Australien und Neuseeland mit seiner Buchclubidee 1986 in weitere internationale Buchmärkte ein. ${ }^{301}$

\subsubsection{4 Öffnung und Refokussierung (1990-2007)}

Die letzte Phase der Bertelsmann Buchclubgeschichte wurde im Wesentlichen von zwei Strömungen geprägt. Auf der einen Seite standen die Bemühungen einer konzeptionellen Öffnung durch die Abweichung von der originären Club-Geschäftsidee, auf

300 Vgl. Lehning, T. (2003), S. 55-61.

301 Vgl. BUA-UP3/44-7 (Lesefamilie, 1988), Anhang; Tietz, B. (1985), S. 353. 
der anderen Seite erfolgte eine Rückbesinnung auf die tradierten Säulen der BuchclubKonzeption.

In Ergänzung zur Buchgemeinschaft wurde im Jahr 1996 die Medienversandfirma Best'Seller gegründet, welche durch ein fehlendes Mitgliedschaftssystem keine Buchgemeinschaft war, sondern hauptsächlich Bücher, aber auch CDs und Videos über Kataloge vertrieb. Aufgrund des Gesetzes zur Buchpreisbindung konnten aktuelle Bestseller nur zum gebundenen Ladenpreis vertrieben werden. Best'Seller betrieb keine eigene Ladenkette. ${ }^{302}$ Im selben Jahr wurde das Konzept der Buchhandelskette Boulevard vorgestellt und eine erste Filiale in Gelsenkirchen eröffnet. ${ }^{303}$ Mit den als, Wohlfühlbuchhandlungen' bezeichneten Läden stieß Bertelsmann erstmals in das Geschäft des vertreibenden Sortimentsbuchhandels vor. Die Kunden der Boulevard-Kette sollten durch eine einladende und großflächige Ladengestaltung zum Buchkauf motiviert werden; das Angebot des Bertelsmann Clubs wurde in die Verkaufsfläche integriert. ${ }^{304}$ Schließlich wurde im Jahr 2001 die bereits 1999 gegründete Online-Plattform zum Medienvertrieb $B O L$ als eigenständiges Profit-Center in den Buchclub integriert. Über $B O L$ konnten Nicht-Clubmitglieder sowie Mitglieder gleichermaßen via Internet aus einem umfangreichen Buch-, CD-, Video- und Unterhaltungsprogramm ohne Kaufverpflichtung frei wählen. ${ }^{305}$

Der strategischen Variabilität von Best'Seller, Boulevard und BOL in den 90er Jahren stand die Refokussierung auf das angestammte Buchclub-Geschäftsfeld in den darauf folgenden Jahren entgegen. In diesem Sinne wurden die Geschäftsaktivitäten

302 Vgl. WIC (1996/08). Im Jahr 1998 wurde zusätzlich die Internet-Plattform ,Boulevard Online’ implementiert. Fortan konnte neben dem stationären Kauf auch online bestellt werden. Vgl. WIC (1998/02).

303 Vgl. Börsenblatt (1996, 12.11.), S. 5.

304 Vgl. WIC (1996/11); WIC (1997/11).

305 Vgl. WIC (2001/05). 
von Best!'Seller nach drei Geschäftsjahren im Jahr 1999 wieder eingestellt. ${ }^{306} \mathrm{Im}$ Jahr 2004 wurde ein erneuter Testlauf gefahren und erstmals nach fünfjähriger Pause ein neuer Katalog aufgelegt. Best'Seller firmiert seitdem unter dem Untertitel ,Der aktuelle ResteSeller' und spezialisiert sich auf Titel des modernen Antiquariats - analog zum ursprünglichen Best!Seller-Modell ohne Mitgliedschaft und Kaufverpflichtung. ${ }^{307}$ Das Boulevard-Konzept wurde nach der Dauer von einem Geschäftsjahr aufgegeben und die bestehende Ladengeschäftskette verkauft. Im Jahr 2002 erfolgte ein erneuter Test des überarbeiteten Boulevard-Konzepts in Kooperation mit einem lokalen Bucheinzelhändler. ${ }^{308}$ Auch dieses Konzept wurde mit dem Verkauf der Boulevard-Buchhandlung im Jahr 2003 verworfen. ${ }^{309}$ Die erst im Jahr 2001 in das Clubgeschäft integrierte Verkaufsplattform $B O L$ wurde ebenfalls nach einem Jahr in 2002 veräußert. ${ }^{310}$

Weitere strategische Erweiterungen des Clubs Bertelsmann waren die versuchte Eta-blierung unterschiedlicher Spezialclubs. Neben den bestehenden Clubs P.P. Kelen, Club top 13 und Hallo RTL wurde ab 1999 in Anlehnung an die USA und England eine Vielzahl weiterer Clubs (z. B. exklusiv für Frauen oder Kinder) diskutiert. ${ }^{311} 2001$ begann der Test für Moments, einem Spezialclub für „Frauen mit einer Vorliebe für gefühlvolle Liebesliteratur". ${ }^{312}$ Nach einem Jahr wurde das Konzept verworfen und der aktuelle Bestand von 10.000 Moments-Mitgliedern in den Bertelsmann Universalclub überführt. $^{313}$

Vgl. BuchMarkt (1999/09).

Vgl. WIC (2004/01), S. 2.

Vgl. BuchMarkt (2001/10); Börsenblatt (2001, 30.11.), S. 8-11.

Vgl. BuchMarkt (2003/02); Buchreport (2003, 25.09.).

Vgl. WIC (2002/08), S.1; FTD (2002, 22.10.).

In den USA existierten z.B. der ,Science Fiction Book Club', das ,Fireside Theatre' oder die ,Mystery Guild'. In Großbritannien behandelten Spezialclubs Themen wie ,Mystery und Thriller', ,Geschichte', ,Kunst', ,Natur', ,Luftfahrt', ,Eisenbahn', „Militaria', ,Kochen', ,Cricket' sowie ,Kings and Queens'. Vgl. BUA-UP3-44/7 (Lesefamilie, 1988), S. 22.

WIC (2001/04), S. 3; Börsenblatt (2002, 26.07.), S. 5.

Vgl. Buchreport (2003/25.09.). 
Organisatorisch prägten zwei grundlegende Restrukturierungen die Zeit von 1990 bis 2007. Zum einen wurde Anfang der 90er Jahre die Konzernstruktur schrittweise nach Produktlinien aufgebaut. Neben den Linien ,BMG Entertainment', ,Industrie’ und ,Presse' wurden die Buchclubs zusammen mit den Buchverlagen und Fachinformationen unter der Produktlinie ,Buch' in der Buch $A G$ zusammengeführt. ${ }^{314}$ Zum anderen erfolgte im Jahr 2000 schließlich die organisatorische Trennung der Clubs von den Verlagen. In der neu geschaffenen Konzernsparte DirectGroup wurden sämtliche direkt an den Kunden vertriebenen Medienprodukte gebündelt. Neben Buchund Musikclubs zählen zu der bis heute bestehenden Division auch die weltweiten ECommerce-Aktivitäten von Bertelsmann. ${ }^{315}$

Der Verbreitung des Internets trug auch die Vertriebsstruktur des Clubs Rechnung: 2001 wurde der Internetauftritt des Bertelsmann Clubs gestartet, so dass Mitglieder neben Katalog- und Filialbestellung auch online die Angebote des Clubs wahrnehmen können. ${ }^{316}$

\subsubsection{Der Bertelsmann Konzern als Systemumwelt}

Anhand der vorgestellten Chronologie des Bertelsmann Buchclubs wird bereits deutlich, dass im Verlauf der über fünfzigjährigen Unternehmensgeschichte zahlreiche Geschäftsfelder neben dem Buchclub aufgebaut wurden und heute zum Teil als eigenständige Sparten des Bertelsmann Konzerns agieren. Auch wenn die vorliegende Arbeit als zentralen Analysegegenstand die strategischen Entscheidungen des deutschen Buchclubgeschäfts behandelt, so liegt die Vermutung nahe, dass einzelne Konzerndivisionen und internationale Bertelsmann Buchclubs relevante Kontextfaktoren des Systems ,Buchclub Deutschland' darstellen, zu denen Beziehungen unterhalten werden und so-

\footnotetext{
314 Vgl. BG (1993/94), S. 4-7.

315 Vgl. BG (1999/2000), S. 33.

316 Vgl. WIC (2001/03).
} 
mit in der Pfadanalyse Berücksichtigung finden müssen. Aus diesem Grund wird im Folgenden auf die wesentlichen Schritte in der Entwicklung des Bertelsmann Konzerns eingegangen, vor deren Hintergrund im anschließenden Kapitel 4.3.3 eine konkrete Unterscheidung zwischen Fall, Kontext und Umwelt der vorliegenden Einzelfallstudie vorgenommen wird. Ziel ist es also zunächst, die wesentlichen strategischen Entwicklungsstufen der Konzerndivisionen in ihrer Chronologie einzufangen, um ein möglichst umfangreiches Bild der Konzernentwicklung zu erhalten. Erst auf Basis der Beschreibungen lässt sich verlässlich über die Relevanz einer potentiellen strategischen Beeinflussung des Buchclubs urteilen. In diesem Sinne werden relevante Kontextfaktoren in der Pfadanalyse unter Kapitel 4.4 aufgegriffen und im Zusammenhang mit der strategischen Entwicklung des Buchclubs pfadtheoretisch ausgewertet.

\subsubsection{Arvato: Druck, Dienstleistung, Onlinebetriebe}

Der mit dem Wiederaufbau 1946 neu gegründete technische Betrieb Mohn \& Co. GmbH umfasste zunächst eine Druckerei, Setzerei sowie Buchbinderei, entwickelte sich jedoch schon bald zu einem modernen Industriebetrieb. Ursächlich hierfür war der durch den Erfolg des Leserings 1950 hervorgerufene, stark wachsende Buchbedarf, welcher mit den vorhandenen Druck- und Distributionskapazitäten nicht mehr befriedigt werden konnte.

„Ab 1955 bebaute die 'Technik' daher ein riesiges Gütersloher Grundstück von 225.000 Quadratmetern mit neuen Industrieanlagen für die Buch-, die Tonträgerherstellung sowie die Distribution. Mohn \& Co. verwandelte sich zu einem modernen Industriebetrieb, der [sich] mit seiner Buchbinderei samt Falzmaschinen, einer Smyth-Buchfertigungsstraße, Super M.A.N.-Buchdruckmaschinen mit Buchdruck-Rotation, einer Repro-Abteilung und neuen Offsettdruckmaschinen auf die Massenanforderungen des Leserings einstellte." 317

Die Druckereibetriebe unter Mohndruck wurden im Jahr 1958 um das Schallplattenwerk Sonopress erweitert, hinzu kamen Lager- und Versandleistungen für Buch- und 
Schallplattenclubs durch das 1959 gegründete Kommissionshaus Buch und Ton. ${ }^{318}$ Steigende Aufträge, allen voran die Massenbestellungen aus dem Buchclub, resultierten im Laufe der 60er Jahre in weiteren „Vergrößerungen, Automatisierungen und Technologieinnovationen “319 des Technik-Bereichs. Ende der 60er umfasste der Leistungskatalog von Mohn \& Co. GmbH neben der Buch- und Schallplatten-Herstellung bereits ein breites Angebotsspektrum von Verpackung, Versand und Lagerhaltung.

Das zunehmende Wachstum der technischen Betriebe und die Expansion in ausländische Märkte führte im Jahr 1971 schließlich zu einer organisatorischen Ausgliederung aus der Bertelsmann-Zentrale zu einem eigenen Unternehmensbereich Technik. ${ }^{320}$ Mit weiter steigender Größe wurde der Bereich Technik im Jahr 1977 in den Unternehmensbereich Druck- und Industriebetriebe (UDI) umbenannt, welcher wiederum die Geschäftsfelder ,Ausland' (Papierfabriken und Druckereien), ,Inland' (Offsetdruck und Dienstleistungen), ,Tiefdruck' und ,Fertigung von Audio- und Videoproduktionen' umfasste. ${ }^{321}$

Bis zum Jahr 1993 wuchs UDI zu einem der weltweit führenden Dienstleister im Druckereigeschäft an, der sich zunehmend auch auf die Immaterialisierung von Daten und die damit verbundenen medialen Veränderungen einstellte. Noch im selben Jahr 1993 erfolgte gemäß dem auf Konzernebene verfolgten Produktlinienkonzept die Umbenennung zu Bertelsmann Industrie. ${ }^{322}$ Hierzu zählte auch der stetig wachsende Bereich der Dienstleistungsunternehmen von Bertelsmann Distribution, der Logistik-

318 Das ,Kommissionshaus Buch und Ton' wurde 1971 in ,Vereinigte Verlagsauslieferung' (VVA) umbenannt und ist für den Versand der unterschiedlichen Frachtgüter zuständig. Vgl. Adron, L. (1983).

319 Lehning, T. (2004), S. 266.

320 In dem Bereich Technik „wurden die graphischen Großbetriebe, die Schallplattenfabrik Sonopress, die Papierfabrik, die Dienstleistungsfirmen sowie die Auslandsniederlassungen zusammengefasst." Lehning, T. (2004), S. 267.

321 Vgl. BG (1977/78), S. 23-28.

322 Produktlinien fassen Bereiche mit "gleicher oder ähnlicher Produkt- und Marktorientierung zusammen." BG (1993/94), S. 4. Buch, BMG Entertainment sowie Gruner+Jahr (Produktlinie Presse) bildeten die übrigen Produktlinien. 
zentren unterhielt und Direktmarketingleistungen wie die Erstellung von Karten- und Clubsystemen oder den Adressverkauf anbot. ${ }^{323}$ Bertelsmann Industrie wurde schließlich 1999 zu der bis heute bestehenden Division Arvato umbenannt. ${ }^{324}$ Neben Offsetund Tiefdruck (arvato print), Logistik, Adress- und Datenbankmanagement umschließt diese Sparte heute auch Servicecenter, Finanzdienstleistungen (arvato services), Informationstechnologie im Internet, Wissensmanagement (arvato systems) sowie ein breites Leistungsspektrum im Bereich digitaler Daten (arvato digital services). ${ }^{325}$

Aufgrund der Funktion als interner Zuliefererbetrieb für den Buchclub lässt sich schließen, dass die Druckerei- und Logistikleistungen der heutigen Division Arvato im Verlauf der Konzerngeschichte eine nicht unwesentliche Rolle für die strategischen Entscheidungen im Buchclub gespielt haben. In welcher Form Geschäftsbeziehungen unterhalten werden und welchen Einfluss die Sparte auf die Buchclubaktivitäten ausübten bzw. noch immer ausüben, wird ausführlich in der Pfadanalyse unter Kapitel 4.4 behandelt.

\subsubsection{Random House: Buchverlage}

Ebenso wie bereits bei den Druckereibetrieben veränderte die Gründung des Leserings in den 50er Jahren auch die Verlagsarbeit von Bertelsmann: „Nicht mehr der Verlag trieb die Organisation an, sondern er wurde angetrieben durch den Erfolg des expansiven Buchclubs, der hohe Auflagenstärke garantierte und die eigene Verlagsleistung von 4.000 Bänden pro Tag überforderte.“" ${ }^{326}$

Entsprechend der gestiegenen Komplexität der Lesering-Aktivitäten erfolgte 1955 eine erste Reorganisation des bislang als eine organisatorische Einheit aufgestellten C. Bertelsmann Verlags und dem daraus entstandenen Lesering. Durch die Gründung

Vgl. Lehning, T. (2004), S. $271 \mathrm{f}$.

Vgl. BG (1998/99), S. 13.

Vgl. Arvato.com (2008).

Lehning, T. (2004), S. 86. 
der Bertelsmann Lesering GmbH wurde die Buchgemeinschaft organisatorisch von Verlag und Vertriebsgemeinschaft getrennt. Der dadurch entstandene Verlagsbereich C. Bertelsmann erfuhr im Jahr 1959 unter dem Dach der C. Bertelsmann KG schließlich eine Neuordnung der bestehenden Einzelfirmen in drei Hauptsegmente:

Im Sigbert Mohn Verlag erschienen fortan schwerpunktmäßig Belletristik- und Jugendbuchtitel, das Gütersloher Verlagshaus Gerd Mohn konzentrierte sich auf theologische Literatur, wohingegen der C. Bertelsmann Verlag vornehmlich Sachbücher und Fachzeitschriften herausgab. ${ }^{327}$ Zur Programmerweiterung wurde zusätzlich im selben Jahr 1959 der Hamburger Mosaik Verlag erworben. ${ }^{328}$

Die frühen 60er Jahre wurden von weiteren Verlagszukäufen, -gründungen, jedoch auch -aufgaben bestimmt. Auf belletristischer Seite erwarb Bertelsmann den Rütten \& Loening sowie den Marion von Schröder Verlag, der Verlag Wissenschaft und Politik sowie der Signum Verlag wurden gegründet, wohingegen der Sigbert Mohn Verlag aufgegeben wurde. ${ }^{329}$

Aufgrund weiterer Verlagszukäufe in den folgenden Jahren fand 1968 eine erneute Neugliederung der Verlagshäuser von Bertelsmann statt. Es wurde die Verlagsgruppe Bertelsmann gegründet, unter welcher alle bisherigen Eigenverlage - mit Ausnahme des Gütersloher Verlagshauses Gerd Mohn - zusammengefasst und neu gegliedert wurden. ${ }^{330}$ Diese Reorganisation unter der Verlagsgruppe gewährleistete die rechtliche und

Vgl. BUA-UP3/26-7 (Bertelsmann Prospekt, 1959), S. 7-13.

Vgl. Bertelsmann (1985), S. 403.

Vgl. Langenbucher, W.R. (1985), S. 44.

Unter dem Dach der Verlagsgruppe bestanden der C. Bertelsmann Verlag (Schöngeistiger Verlag), der Bertelsmann Sachbuchverlag, der Bertelsmann Lexikonverlag, das Kartographische Institut Bertelsmann, der Bertelsmann Fachverlag, der Bertelsmann Jugendbuchverlag, der Bertelsmann Kunstverlag, der Bertelsmann Universitätsverlag, der Bertelsmann Ratgeberverlag, der Verlag für Buchmarktforschung Hamburg sowie der Reise- und Verkehrsverlag Stuttgart. Vgl. BI (1968/01), S. 7; BI (1969/01), S. 6; BI (1969/02), S. 4 ; BI (1969/03), S. 8f. ; BI (1969/04), S. 2f. Für das detaillierte Organigramm der Verlagsgruppe Bertelsmann siehe BI (1968/01), S. 7. 
programmatische Selbständigkeit der einzelnen Verlage,, ${ }^{331}$ wohingegen ein Großteil der kaufmännischen Aufgaben der Verlagsgruppe selbst zufiel. ${ }^{332} 1972$ erfolgte der Umzug der Verlagsgruppe Bertelsmann von Gütersloh nach München, in den darauf folgenden Jahren eine Vielzahl von Zukäufen wie z. B. die Übernahme des Blanvalet Verlags 1975. ${ }^{333}$ Der Eintritt in den deutschen Taschenbuchmarkt wurde im Jahr 1977 durch die Akquisition des Wilhelm Goldmann-Verlags geleistet. ${ }^{334}$

Kurze Zeit später verstärkte Bertelsmann die Expansion in ausländische Märkte, zunächst durch eine Beteiligung an dem spanischen Verlagshaus Plaza \& Janés, der italienischen Verlagsgruppe Fratelli Fabri Editore ${ }^{335}$ sowie an dem größten USamerikanischen Taschenbuchverlag Bantam Books.336 Im Jahr 1986 festigte Bertelsmann schließlich seine Position im amerikanischen Buchmarkt durch den Kauf des zweitgrößten US-amerikanischen Verlags Doubleday+Co.Inc, zu welchem auch der Taschenbuchverlag Dell/Delacorte gehört. ${ }^{337}$ Der stetig wachsende Unternehmensbereich der Verlage führte im Jahr 1988 zu einer Neustrukturierung des Buchbereichs:

Die drei neu gegründeten Unternehmensbereiche, Internationale Buch- und Schallplattengemeinschaften', ,Buch Deutschland, Österreich, Schweiz' (hierunter fiel auch der deutsche Buchclub) und, Verlagsgruppe Bertelsmann International' gingen aus den Bereichen ,Buch- und Schallplattengemeinschaften' und ,Verlage' hervor und festigten organisatorisch die Bindung zwischen Verlagswesen und Buchgemeinschaften. ${ }^{338}$

Vgl. Gööck, R. (1968), S. 214; SZ (1988, 21.10.).

„Die Zentrale der Verlagsgruppe übernimmt dagegen alle Aufgaben, die sich von leistungsfähigen Spezialistengruppen besser ausführen lassen - beispielsweise den Vertrieb mit Verkauf und Werbung, die Buchgestaltung und -herstellung, die Kalkulation und Abrechnung, das Lizenzwesen und die Pressearbeit." Gööck, R. (1968), S. 214.

Vgl. Langenbucher, W.R. (1985), S. 46; Die Zeit (1981, 22.05.).

Vgl. Lehning, T. (2004), S. 95-97.

3351980 wurde die Beteiligung an der Verlagsgruppe Fratelli Fabri Editore wieder veräußert. Vgl. Langenbucher, W.R. (1985), S. 54.

336 Vgl. Langenbucher, W.R. (1985), S. 53.

337 Vgl. FAZ (1986, 27.09.).

338 Vgl. BG (1988/89), S. 6; BG (1992/93), S. 34-36; SZ (1989, 05.12.). 
Eine erneute Reorganisation wurde im Geschäftsjahr 1991/92 mit der Neugliederung der Buchunternehmen unter der Produktlinie ,Buch', in der sämtliche Buch-, Verlags- und auch Clubgeschäfte zusammengefasst wurden, vollzogen, so dass erstmals seit 1955 die Buchclubaktivitäten und Eigenverlage wieder in einer organisatorischen Einheit zusammengefasst wurden. ${ }^{339}$ Aus der Produktlinie ,Buch' ging schließlich im Geschäftsjahr 1993/94 die Buch $A G$ hervor, unter welcher die Geschäftsaktivitäten aus den Bereichen Buch Deutschland, Österreich, Schweiz und der Verlagsgruppe Bertelsmann International gebündelt wurden. ${ }^{340}$ Die Übernahme des größten Verlags der englischsprachigen Literatur Random House im Jahr 1998 zog schließlich weitere Reorganisationen nach sich. ${ }^{341}$ Zunächst trennte sich der Bereich Fachinformation von den belletristischen Verlagen und wurde als eigenständiges Geschäftsfeld aus der Buch $A G$ ausgelagert. ${ }^{342}$ Der in 1999 akquirierte wissenschaftliche Springer-Verlag wurde in den Bereich Fachinformation integriert und seitdem als Unternehmenssparte Bertelsmann Springer Science + Business Media (BertelsmannSpringer) geführt. ${ }^{343}$ Schließlich wurden nach Auflösung der Buch $A G$ im Geschäftsjahr 2000/01 sämtliche BertelsmannVerlage in der neu geschaffenen Gruppe Random House zentralisiert. ${ }^{344}$ Während die Verlagsgruppe Random House bis heute besteht, wurde BertelsmannSpringer im Jahr 2003 veräußert. ${ }^{345}$

Ähnlich wie im Fall von Arvato lässt auch die strategische Entwicklung der Verlagsgruppe einen engen Bezug zu den Buchclubaktivitäten von Bertelsmann erkennen, da die Eigenverlage durch den Verkauf von Lizenzen zu wesentlichen Teilen von dem Erfolg des Buchclubs profitierten. Parallel zum Wachstum des Clubs expandierte auch 
die Verlagsgruppe in beträchtlichem Maß durch Zukäufe und Neugründungen. In welcher Form die Entwicklungen der Verlagsgruppe auf die strategischen Entscheidungsprozesse des Buchclubs Einfluss genommen haben, behandelt ausführlich die Pfadanalyse in Kapitel 4.4.

\subsubsection{Gruner+Jahr: Zeitschriften und Zeitungen}

Bertelsmann - traditionell als Verlagshaus mit Druckereibetrieb und Buchgemeinschaft tätig - drang bereits früh in den Zeitschriftenmarkt ein. In engem Zusammenhang mit dem Lesering wurde erstmals 1955 die Zeitschrift Bertelsmann Drei herausgegeben, jedoch bereits drei Jahre später wieder eingestellt. Der eigentliche Einstieg in das Zeitschriftengeschäft lässt sich auf das Jahr 1969 datieren, indem Bertelsmann $25 \%$ Anteile an dem Hamburger Verlagshaus Gruner+Jahr $r^{346}$ erwarb. Bereits vier Jahre später wurde Bertelsmann durch eine erneute Beteiligung auf insgesamt $60 \%$ zum Mehrheitseigner, ${ }^{347} 1975$ wurden weitere 9,9\% Anteile erworben, 1976 erfolgte die bis heute maßgebliche Aufstockung um $5 \%$ auf 74,9\%.348 Das Produktportfolio erweiterte sich zunächst sukzessive im Inland durch Zukäufe und die Entwicklung eigener Titel, ${ }^{349}$ ab dem Jahr 1978 erfolgte schließlich die Expansion in ausländische Märkte.

1984 stieß Gruner+Jahr durch die Beteiligung an der UFA-Film- und Fernsehen $G m b H$, zu der u. a. die TV-Sender RTL und VOX gehörten, erstmals in den Bereich der elektronischen Medien vor. ${ }^{350}$ Fernsehformate wie tv magazin oder Stern TV sowie die Einflussnahme auf Radioprogramme ermöglichten fortan eine multimediale

Gruner+Jahr wurde 1965 von Gerd Bucerius (28,25\%), John Jahr (32,25\%) und Richard Gruner (39,5\%) gegründet und verlegte zu diesem Zeitpunkt bereits den Stern, die Zeit, Constanze, Brigitte, Petra und Schöner Wohnen.

347 Vgl. Bertelsmann.de (2008a).

348 Vgl. Bertelsmann.de (2008b); Langenbucher, W.R. (1985), S. 51.

349 Ein prominentes Beispiel hierfür ist die Eigenentwicklung der Zeitschrift Geo mit den Derivaten Geo Special, Geo Wissen, Geo Season, Geolino, Geo Epoche sowie den Geo Büchern.

$350 \quad$ Vgl. SZ (1984, 25.06.). 
Markenverbreitung. Parallel dazu betrat Gruner+Jahr den Tageszeitungsmarkt durch den Kauf der Hamburger Morgenpost (1986), des Berliner Verlags (vollständige Übernahme 1992) 351 sowie des Dresdner Druck- und Verlagshauses (vollständige Übernahme 1994). ${ }^{352}$ Schließlich wurde 2000 in einem Joint Venture mit dem britischen Medienkonzern Pearson die überregionale Tageszeitung Financial Times Deutschland (FTD) neu gegründet. Heutzutage ist Gruner+Jahr neben seinen Geschäftsaktivitäten im Zeitungs- und Online-Informationsbereich zu dem größten Zeitschriftenhaus in Europa geworden. ${ }^{353}$

Die Chronologie der Zeitschriften- und Zeitungsgeschäfte der heutigen Division Gruner+Jahr lassen den Schluss zu, dass auf Konzernebene eine breite Aufstellung im Medienmarkt verfolgt wird. Enge strategische Berührungspunkte mit der Entwicklung des Buchclubgeschäfts ließen sich nicht feststellen, so dass die Division Gruner+Jahr in der Pfadanalyse keine weitere Berücksichtigung finden wird.

\subsubsection{Sony BMG: Musikvermarktung}

Seinen ersten Schritt in den Tonträgermarkt unternahm Bertelsmann bereits fünf Jahre nach Gründung des Leserings durch eine Angebotserweiterung um Schallplatten. Gemäß des Mottos ,Zum guten Buch gehört der schöne Klang' gründete Bertelsmann 1956 einen eigenen Schallplattenring, dessen Mitgliedsbedingungen sich an denen des Leserings anlehnten. Basierend auf dem technischen und vertrieblichen Know-how des Druckereibetriebs der Mohn \& Co. GmbH eröffnete Bertelsmann zwei Jahre später sein eigenes Schallplattenpresswerk Sonopress, im selben Jahr folgte die Gründung der Schallplattenproduktionsfirma Ariola. ${ }^{354}$ Aufgrund der großen Schwierigkeiten, die

\footnotetext{
351 Auch der Berliner Verlag wurde im Jahr 2002 an die Verlagsgruppe Holtzbrinck verkauft. Vgl. BG (2002), S. 25.

352 Vgl. Lehning, T. (2004), S. 152.

353 Vgl. Guj.de (2008).

354 Vgl. hierzu ausführlich Kapitel 4.3.1.2 dieser Arbeit.
} 
Titellizenzen populärer Künstler zu erwerben, war es Bertelsmann so möglich, unter der Ariola Eurodisc Musikproduktion auch einen eigenen Interpretenstamm aufzubauen und selbständig zu vermarkten. In den folgenden Jahren schloss Ariola zahlreiche Verträge unter anderem mit der Deutschen Grammophon-Gesellschaft, der sowjetischen Schallplattenfirma Melodia sowie der Hansa Musikproduktion ab und konnte sein Hauptrepertoire um den Deutschen Schlager mit Klassik-, Unterhaltungs-, und Popmusik abrunden. ${ }^{355}$

Ab Mitte der 70er Jahre forcierte Ariola die Expansion in ausländische Märkte und startete seine Geschäftsaktivitäten in weiteren westeuropäischen, lateinamerikanischen sowie US-amerikanischen Staaten.

Die wachsende Musiksparte des Bertelsmann Konzerns ging schließlich mit einer Reorganisation einher, so dass im Jahr 1977 neben den nationalen auch sämtliche internationale Musikunternehmen von Bertelsmann im Vorstandsbereich ,Musik, Film, Fernsehen' zusammengefasst wurden. Nach einigen Rückschlägen im USamerikanischen Musikgeschäft kaufte Ariola 1980 das amerikanische Label Arista Records, dessen 50 \%-Anteile im Rahmen eines Joint-Ventures 1984 mit dem amerikanischen Großkonzern Radio Corporation of America (RCA) veräußert wurden und RCA/Ariola eine sichere Platzierung auf dem internationalen Tonträgermarkt sicherte. ${ }^{356}$ Zwei Jahre später erwarb Bertelsmann die restlichen Anteile des Ariola/RCAJoint-Ventures ${ }^{357}$ und fasste alle Musik-, Rundfunk- und Tonträgerproduktionsfirmen unter der Sparte Bertelsmann Music Group (BMG) zusammen. ${ }^{358}$ Nach zahlreichen kleineren Unternehmenszukäufen drang Bertelsmann Ende der 80er erstmals durch

355 Bereits 1971 zählte Ariola-Eurodisc zu den sieben größten Schallplattenfirmen in Westdeutschland mit namhaften Stars wie Peter Alexander, Heintje, Udo Jürgens oder Mireille Mathieu. Vgl. Langenbucher, W.R. (1985), S. 50f.

356 Vgl. BG (1984/85), S. 8; Langenbucher, W.R. (1985), S. 51.

357 Vgl. FAZ (1986, 10.09.).

358 Vgl. BG (1985/86), S. $10 f$. 
ein Joint Venture mit der Japan Victor Company (JVC) in den asiatischen Markt ein. Es folgten die Übernahme von weiteren asiatischen Musiklabels wie Fun House in Japan, Elite Music in Taiwan und Music Impact in Hongkong sowie eine $20 \%$ Beteiligung an der einflussreichen amerikanischen Zomba Music Group im Jahr 1996. ${ }^{359}$ Im Jahr 2001 verleibte sich $B M G$ die Musikorganisation Zomba schließlich zu $100 \%$ ein. ${ }^{360}$ Nach dem erfolglosen Versuch, im Online-Geschäft mit der Filesharing/ Musiktauschbörse napster ${ }^{301} \mathrm{Fuß}$ zu fassen und dem kartellrechtlichen Verbot der Fusion von $B M G$ mit $E M{ }^{362}$ erfolgte im Jahr 2003 die Fusion von $B M G$ mit der Musiksparte des Elektronikkonzerns Sony (Sony Music Entertainment) zur Bertelsmann Divison Sony BMG, an welcher beide Konzerne bis heute zu je 50\% beteiligt sind. ${ }^{363}$

Zusammenfassend lässt sich festhalten, dass die Ursprünge der heutigen Musiksparte Sony BMG zwar auf den Schallplattenring und somit die Clubgeschäfte der 50er Jahre von Bertelsmann zurückzuführen sind, sich die strategische Entwicklung der Musikgeschäfte jedoch im Verlauf der Unternehmensgeschichte zunehmend verselbständigt hat. Von einer starken gegenseitigen Beeinflussung von Sony BMG und Buchclub ist somit nicht auszugehen, so dass auch die Musiksparte von Bertelsmann nicht Gegenstand der anschließenden Pfadanalyse sein wird.

\subsubsection{RTL-Group: Film, Fernsehen, Hörfunk}

In den Film- und Fernsehmarkt zog Bertelsmann erstmals im Jahr 1960 mit der damals noch sehr kleinen Fernsehproduktionsgesellschaft (BFP) ein. In enger Zusammenarbeit mit den öffentlich-rechtlichen Sendeanstalten wurden mit der BFP unter anderem Fernsehspiele und Dokumentationen hergestellt.

\footnotetext{
359 Mitte der 90er Jahre zählten über 200 Labels, Musikverlage, Filmproduktionen, Musikclubs und Tonträgerhersteller zur Bertelsmann Music Group.

360 Vgl. BG (2002), S. 28f.; FTD (2002, 12.06.), S. 3.

361 Vgl. hierzu ausführlich Röttgers, J. (2003), S. 17-45.

362 Vgl. FAZ (2002, 19.10.), S. 14.

363 Vgl. BG (2004), S. 32f.
} 
Das Engagement von Bertelsmann im Filmmarkt steigerte sich beträchtlich mit dem Erwerb des Filmkonzerns Universum Film AG (UFA) im Jahr 1964. ${ }^{364}$ Unter dem Dach der UFA vereinte Bertelsmann fortan die UFA International (FilmExportfirmen), UFA-Werbefilm (Kinowerbefilme, Fernsehspots etc.), UFAFilmauswertung, die Deutsche Wochenschau GmbH, den Bohème und Tonverlag sowie kurze Zeit später die UFA Theater $A G$ und die Kinos der $U F A .{ }^{365}$ Das erklärte Ziel, zu einem integrierten Film- und Fernsehkonzern zu werden, wurde konsequent durch eine 50 \%-Beteiligung an der Verleih- und Produktionsfirma Constantin-Film - zu der auch die Filmimportgesellschaft Beta gehörte - im Jahr 1965 verfolgt. Im selben Jahr beteiligte sich Bertelsmann am Pallas-Filmverleih, Nora-Filmverleih und an der Merkur-Filmtheatergruppe. ${ }^{366}$

Nachdem die beiden Pläne von Bertelsmann, einen privaten, kommerziellen Fernsehkanal zu gründen und Filme in Kassenform an Endkunden zu vertreiben, scheiterten, zog sich Bertelsmann im Jahr 1970 zu großen Teilen aus dem Filmgeschäft zurück. ${ }^{367}$ Im Jahr 1972 wurden sowohl die Anteile von Constantin-Film als auch an der UFA-Filmtheater $A G$ veräußert. Die verbleibenden Firmen UFA-Werbefilmproduktion und UFA-Fernsehproduktion wurden zusammen mit den übrigen Geschäftsfeldern im Tonträgermarkt (hierzu zählte auch Ariola) in dem Unternehmensbereich ,Musik, Film, Fernsehen' zusammengefasst. ${ }^{368}$

Mehr als zehn Jahre später stieß Bertelsmann erneut in den Film- und Fernsehmarkt vor: Der bevorstehende ,Staatsvertrag zur Neuordnung des Rundfunkwesens' ${ }^{369}$ sollte erstmals die Koexistenz von öffentlich-rechtlichen und privaten Rundfunkgesell-

\footnotetext{
364 Vgl. Bertelsmann.de (2008a).

365 Vgl. Rohloff, A. (2003), S. 70f.

366 Vgl. Gööck, R. (1968), S. 216-218, Rohloff, A. (2003), S. 73.

367 Vgl. UFA (2007), S. 100.

368 Vgl. Langenbucher, W.R. (1985), S. 51.

369 Vgl. Stock, M. (1992), S. 189-221.
} 
schaften erlauben, zudem wurden durch die Verbreitung eines Kabelnetzes die Voraussetzungen für eine technische Umsetzbarkeit privater Rundfunkveranstalter geschaffen. 1983 schloss Bertelsmann mit dem luxemburgischen Unternehmen Compagnie Luxembourgoise de Télédiffusion (CLT) einen Kooperationsvertrag ab und beteiligte sich zu $40 \%$ an dem privaten CLT-Sender RTLplus. ${ }^{370}$

Organisatorisch reagierte Bertelsmann auf die Neuausrichtung im Film- und Fernsehgeschäft durch die Schaffung eines neuen Unternehmensbereichs, Elektronische Medien', zu welchem unter der Leitung der UFA Film- und Fernsehen GmbH die Gesellschaften UFA-Filmproduktion GmbH, Universum-Film GmbH Werner Mietzer sowie die Gruner+Jahr-Firmen Stern TV, GEO-Film International und G+J Filmproduktion zählten. ${ }^{371}$

Das Ziel einer breiten Akzeptanz von RTLplus im deutschsprachigen Raum führte schließlich im Jahr 1986 zur Gründung der RTLplus Deutschland Fernsehen GmbH, an welcher die WAZ-Gruppe, FAZ, Burda und die Kabelmedia-Programmgesellschaft beteiligt wurden. Der steigende Einfluss der werbetreibenden Industrie, ständige Investitionen und immer komplexer werdende Geschäftsaktivitäten bewirkten im Jahr 1993 eine weitere Reorganisation unter der UFA-Holding, innerhalb derer die UFA Media + Marketing mit der Vermarktung von TV-Übertragungsrechten, Spielfilmen und Fernsehproduktionen eine zentrale Stellung einnahm. Nach der Umbenennung von RTLplus zu $R T L^{372}$ erfolgte im Jahr 1993 unter dessen Dach die Gründung des Kanals RTL 2 mit den Bertelsmann-Beteiligungsverhältnissen von 38,9\% an RTL und 7,9\% an RTL 2.

370 Vgl. Handelsblatt (1983, 12.12.); Rohloff, A. (2003), S. $85 f$.

371 Vgl. Langenbucher, W.R. (1985), S. 56. Neben dem Fernsehgeschäft engagierte sich Bertelsmann ebenfalls im Hörfunkbereich: Bertelsmann unterhielt die Rundfunknachrichtenagentur (RUFA), den Deutschen Funk Programm Service ( $d f s$ ) sowie zahlreiche Radiostationen wie z. B. Antenne Bayern und Klassik Radio.

372 Vgl. Rtl-television.de (2008). 
Im selben Jahr setzte Bertelsmann einen weiteren Meilenstein in seinen Fernsehaktivitäten: Zusammen mit der Holtzbrinck-Gruppe, dem Süddeutschen Verlag und der West $L B$ wurde der auf Infotainment für höhere Bildungsschichten ausgerichtete Kanal VOX gestartet. Nachdem sämtliche Partner aufgrund sinkender Quoten ihre Anteile bereits 1994 veräußerten, gelang es Bertelsmann durch Beteiligungen des französischen Senders Canal+ und Rupert Murdoch, den Kanal zu retten und programmatisch umzubauen. Neben VOX engagierte sich Bertelsmann zunehmend bei RTL und erwarb unter der mit der WAZ-Gruppe gegründeten Gesellschaft BWTV mit 50,1\% die Mehrheitsanteile an RTL. Im Jahr 1996 fusionierten CLT und UFA zu dem Gemeinschaftsunternehmen CLT-UFA ${ }^{373}$ und nahmen fortan die „marktführende Position in den Bereichen Fernsehen, Hörfunk und Filmproduktion “ ${ }^{374}$ ein. Als organisatorische Konsequenz der komplexen CLT-UFA-Gruppe wurde diese aus der Sparte ,BMG Entertainment' ausgegliedert und im Jahr 1998 unter der Sektion CLT-UFA eigenständig weitergeführt.

Nach der fast vollständigen Übernahme der VOX-Anteile gründete CLT-UFA im Jahr 2000 zusammen mit der TV-Sparte des britischen Verlagsunternehmens Pearson die RTL-Group mit den vier Säulen TV, Radio, Content und New Media. ${ }^{375}$ Hauptanteilseigner waren Bertelsmann/WAZ (BWTV) mit $37 \%$, GBL mit $30 \%$ sowie Pearson mit $22 \%$. Bereits ein Jahr später veräußerten sowohl GBL als auch Pearson ihre Anteile an den Bertelsmann-Konzern, so dass dieser seit 2001/02 bis heute mit rund $90 \%$ an der RTL-Group Mehrheitseigner ist. ${ }^{376}$ Bertelsmann erweiterte zudem das

Beteiligt waren zu je $50 \%$ Bertelsmann und die Audiofina $A G$, die wiederum zu großen Teilen GBL Electrafina - den ehemaligen Eignern von CLT - gehörte.

374 Lehing, T. (2004), S. 240. „Die CLT-UFA bündelte 22 Fernsehstationen, darunter prominente Marken wie RTL, M6 und Channel 5, die täglich geschätzte 120 Millionen potentielle Zuschauer erreichten." Lehning, T. (2004), S. $240 \mathrm{f}$.

375 Die RTL-Group vereinte 23 TV-Sender in insgesamt neun Ländern.

376 Vgl. Bertelsmann.de (2008c); BG (2000/01), S. 4. 
Portfolio der RTL-Sendefamilie um den Nachrichtensender $n$ - $t v$, an welchem er in 2002 zunächst 47,3 und in 2004 weitere $2,7 \%$ Anteile erwarb. ${ }^{377}$

Auch im Fall der heutigen RTL-Gruppe ist wie bereits bei Gruner+Jahr und Sony $B M G$ keine enge strategische Verflechtung zum Buchclub zu beobachten. Eine Dominanz von Synergiepotentialen ließ sich nicht feststellen. Somit findet auch die strategische Entwicklung der Fernsehaktivitäten von Bertelsmann als nicht relevanter Umweltfaktor in der Pfadanalyse zum Buchclub keine weitere Berücksichtigung.

\subsubsection{Zwischenfazit zur Bertelsmann AG}

Zusammenfassend lässt sich festhalten, dass der Bertelsmann Buchclub auf eine lange Unternehmensgeschichte zurückblickt. 1950 als Vertriebsidee des C. Bertelsmann Verlags entstanden, ist der ,Lesering Das Bertelsmann Buch’ zum Ende der Analyse im Jahr 2007 als ,Der Club' in der Konzerndivision Direct Group der Bertelsmann AG organisiert. Neben zahlreichen Bertelsmann Buchclubgründungen im Ausland entstanden sukzessive weitere Unternehmensbereiche im Gesamtunternehmen Bertelsmann, die als organisatorisch eigenständige Konzernsparten geführt werden.

Wie angekündigt, stellt die folgende Analyse die strategischen Entscheidungsprozesse des deutschen Bertelsmann Buchclubs als fokales System in den Mittelpunkt. Die weiteren im Laufe der Unternehmensgeschichte entstandenen Teilbereiche des Bertelsmann Konzerns bilden Teilbereiche (Umsysteme) der Umwelt. In Anlehnung an den in Kapitel 3.2 vorgestellten Aufbau einer holistischen Einzelfallstudie lassen sich die einzelnen Teilbereiche des Konzerns in relevante Kontextfaktoren und weitere Umweltfaktoren, die in der Einzelfallstudie nicht näher untersucht werden, aufteilen.

Obwohl innerkonzernliche Austauschbeziehungen wie z. B. Cross-MarketingAktivitäten über sämtliche Konzerndivisionen genutzt werden, ${ }^{378}$ ließen sich unter

377 Vgl. BG (2002), S. 17.

378 Vgl. Schulze, B. et al. (2005), S. 138-147. 
Vorgriff auf die nachfolgende Analyse die stärksten strategischen Verknüpfungen des deutschen Buchclubs mit den Divisionen Arvato und Random House feststellen. ${ }^{379}$ Diese flossen - zusammen mit den internationalen Bertelsmann Buchclubs - als relevante Kontextfaktoren mit in die Untersuchung ein. Die weiteren Konzernbereiche, die heute unter den Divisionen RTL, Gruner+Jahr und $B M G$ firmieren, finden in der weiteren Einzelfallbetrachtung keine weitere Beachtung, da deren strategische Entwicklung einen zu geringen Einfluss auf den Verlauf des deutschen Bertelsmann Buchclubs ausübte. Abbildung 11 illustriert den Untersuchungsschwerpunkt der vorliegenden Arbeit.

Abbildung 11: Fokales System ,Buchclub Deutschland' und Kontextfaktoren der Bertelsmann AG

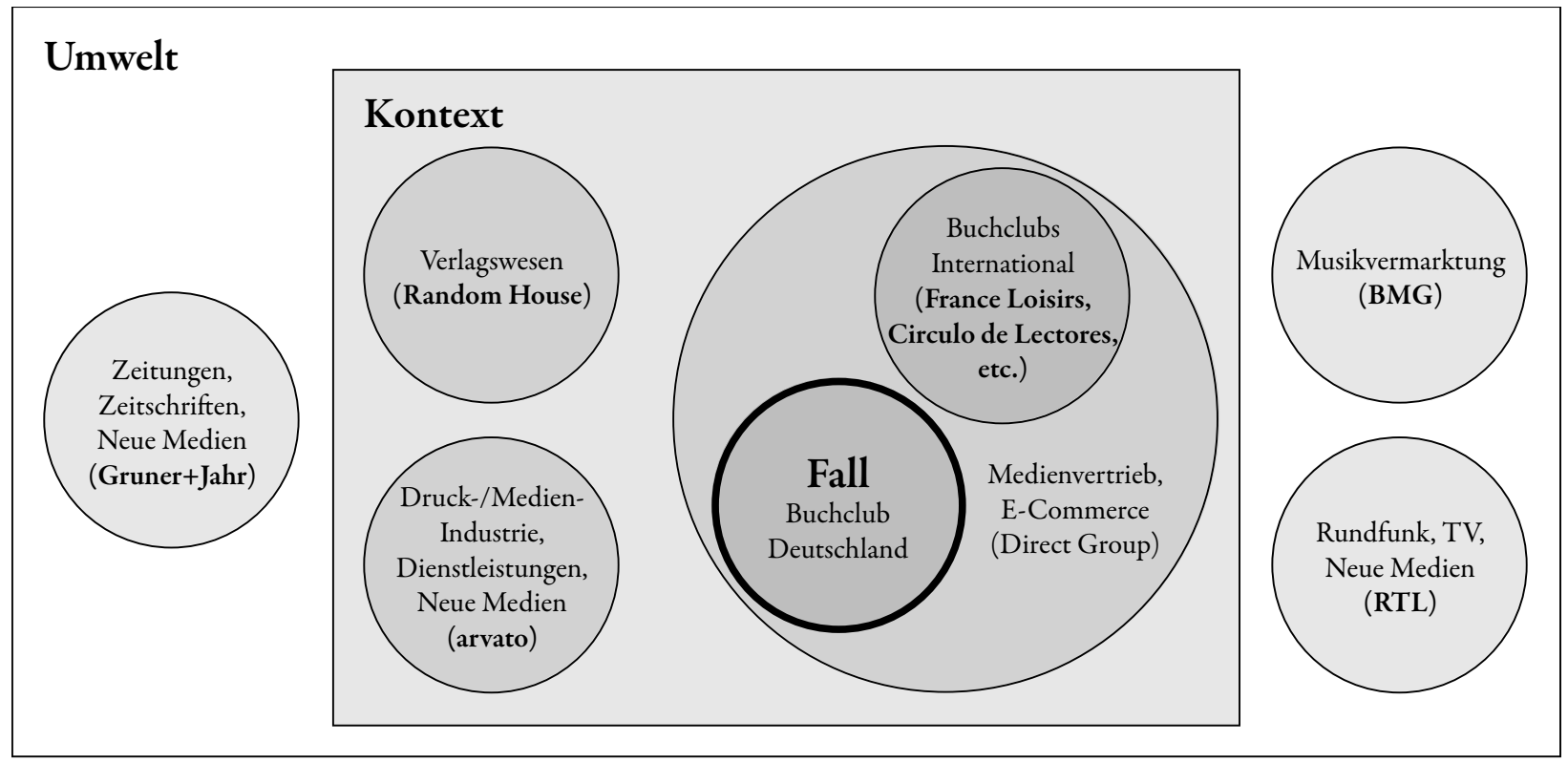

Quelle: Eigene Darstellung in Anlehnung an Bertelsmann.de (2008d). 


\subsection{Pfadtheoretische Analyse der strategischen Entwicklung}

Im nun folgenden Kapitel werden die Ergebnisse der empirischen Datenauswertung vorgestellt. Wie im einleitenden Kapitel 1 ausführlich erläutert, steht die Analyse unter der zentralen Forschungsfrage, warum ein Unternehmen langfristig an verlustreichen Strategien festhält. Hierzu werden die strategischen Entscheidungsprozesse des deutschen Bertelsmann Buchclubs von 1950 bis 2007 anhand der zentralen pfadtheoretischen Annahmen geprüft. Diese Analyse gliedert sich gemäß der vorgestellten Konzeption aus Kapitel 2.5 in zwei grundlegende Schritte:

Schritt 1 untersucht, ob die strategische Ausrichtung des Bertelsmann Buchclubs im Zeitverlauf rigide ist. Erhärtet sich der Rigiditätsverdacht, schließt sich in Schritt 2 die Pfaddiagnose an, anhand derer die zentralen Komponenten der Pfadtheorie am Fall des Bertelsmann Buchclubs diskutiert werden.

Um diese Erhebung durchzuführen, ist es jedoch zunächst notwendig, aus den einzelnen pfadtheoretischen Komponenten messbare Indikatoren abzuleiten. Mit diesem Operationalisierungsprozess befasst sich das nun folgende Kapitel.

\subsubsection{Operationalisierung der Pfadelemente}

In Anlehnung an die Konzeption strategischer Pfadabhängigkeit (Kapitel 2.5) soll in Schritt 1 geprüft werden, ob strategische Rigidität vorliegt. Strategische Rigidität wiederum spaltet sich in die Elemente der strategischen Kontinuität und der dynamischen Umweltbedingungen auf. Für beide Elemente gilt es, messbare Indikatoren zu finden.

Unter Rückgriff auf die begriffliche Festlegung von Unternehmensstrategien in Kapitel 2.2.2 müssen allein bei der Operationalisierung des ersten Elements ,strategische Kontinuität' drei Ebenen Berücksichtigung finden: die Ebene des Geschäftsmodells, der Corporate Strategy sowie der Business Strategy. Alle Ebenen sollen bezüglich Stabilität bzw. Variabilität untersucht werden, um verlässliche Aussagen über den stra- 
tegischen Verlauf einer Organisation zu erhalten. Es liegt strategische Kontinuität vor, sofern die inhaltliche Ausgestaltung der einzelnen Strategiedimensionen über den Analysezeitraum konstant bleibt.

Nach der Bestimmung der strategischen Entwicklung im Zeitverlauf gilt es, die parallel stattfindende Umweltentwicklung als zweites Element strategischer Rigidität abzubilden. In Anlehnung an PORTER (1999) sowie ergänzend an STEINMANN/ SCHREYÖGG (2005) eröffnet sich ein sechsteiliges Raster als Analyserahmen zur Identifikation der Wettbewerbsentwicklung innerhalb der Branche als ,Mikroumwelt': Potentielle Neuanbieter, Abnehmer, Lieferanten, Substitutionsprodukte, Rivalität unter den Konkurrenten sowie industrielle Beziehungen und der Staat als Wettbewerbsfaktoren. ${ }^{380}$

Hierbei sprechen erhöhter Wettbewerb durch neue Konkurrenten und niedrige Markteintrittsbarrieren, zunehmende Verhandlungsmacht von Abnehmern und Lieferanten, die steigende Zahl funktional äquivalenter Ersatzprodukte, verstärkte Rivalität unter den bestehenden Wettbewerbern (z. B. durch Marktkonzentrationsprozesse) sowie staatliche Einflussnahme für eine Verschärfung und somit Dynamisierung der Marktbedingungen. Auch die gegenteilige Entwicklung - eine Entschärfung der Wettbewerbsverhältnisse - ist möglich und unterstreicht den Wandel in der jeweiligen Branche. Nachfolgende Tabelle 10 fasst die einzelnen Indikatoren, anhand derer strategische Kontinuität und Umweltdynamik messbar sind, zusammen. 
Tabelle 10: Operationalisierung strategischer Rigidität als Schritt 1 der Pfadanalyse

\begin{tabular}{|c|c|c|c|}
\hline \multirow{3}{*}{ 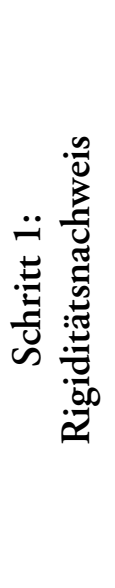 } & Konstrukt & Elemente & Indikatoren \\
\hline & \multirow[b]{2}{*}{ Strategische Rigidität } & Strategische Kontinuität & $\begin{array}{l}\text { - Geschäftsmodell } \\
\text { - Corporate Strategy } \\
\text { - Business Strategy }\end{array}$ \\
\hline & & Dynamische Umwelt & $\begin{array}{l}\text { - Neuanbieter } \\
\text { - Abnehmer } \\
\text { - Lieferanten } \\
\text { - Substitutionsprodukte } \\
\text { - Konkurrenten } \\
\text { - Staat }\end{array}$ \\
\hline
\end{tabular}

Quelle: Eigene Darstellung.

Sofern ein Rigiditätsbefund vorliegt, gilt es in Schritt 2, die strategischen Entscheidungsprozesse konkret auf das Phänomen der Pfadabhängigkeit hin zu analysieren. Hierzu wurden auf Basis der zentralen pfadtheoretischen Annahmen die vier Komponenten Historiztität, Critical Juncture, positive Rückkopplung und Lock-In extrahiert. Für deren einzelne Elemente müssen zur empirischen Erhebung ebenfalls adäquate, messbare Indikatoren gefunden werden.

Die Pfadkomponente ,Historizität' fußt auf den beiden Elementen ,Kontingenz' und ,Vorprägung'. Kontingenz zeigt sich in Form von potentieller Entscheidungspluralität und Ergebnisoffenheit. Ein zukünftiger Entscheidungsverlauf darf unter kontingenten Bedingungen noch nicht determiniert sein, sondern muss die Option unterschiedlicher Entscheidungswege bereithalten. Die Potentialität des Indikators ,Entscheidungspluralität' deutet bereits auf die Schwierigkeit einer Messung hin, lässt sich doch retrospektiv nur der tatsächlich gewählte Entscheidungsprozess beobachten. Als Ausweg bietet sich die Untersuchung unterschiedlich diskutierter Alternativen sowie die Beobachtung von konkurrierenden Organisationen als Benchmarks an, die sich in einer vergleichbaren Situation abweichend verhalten haben. Als Indikator für das zweite Element der Historizität - ,Vorprägung' - sollen Imprints erhoben werden. 
Diese umfassen zeitlich vorgelagerte Kontextfaktoren, die auf die zu untersuchende Entscheidungssituation ausstrahlen, sie jedoch nicht vollständig determinieren.

Die zweite zu messende Pfadkomponente des Critical Junctures, basierend auf Small Events, lässt sich an denjenigen Ereignissen bzw. Entscheidungen messen, deren Wirkung auf das anschließende Entscheidungsverhalten nachhaltigen Einfluss ausübt. Konkret indizieren diejenigen Ereignisse ein Critical Juncture, die den nachfolgenden Mechanismus der positiven Rückkopplung auslösen.

Zur Messung der positiven Rückkopplung steht bereits ein breites Spektrum unterschiedlicher Selbstverstärkungsformen zur Verfügung. Wie Tabelle 2 zeigte, ist konkret nach Economies of Scale and Scope, direkten und indirekten Netzexternalitäten, Lernen, adaptiven Erwartungen, Koordinations- sowie Komplementaritätseffekten zu suchen. Die konkrete Erhebung der unterschiedlichen Selbstverstärkungsmechanismen stellt aufgrund ihres noch immer hohen Abstraktionsgrads sowie der vielfältigen Ausprägungsmöglichkeiten eine besonders große Herausforderung dar.

Erstens können - wie in Kapitel 2.3.2.2.2 ausführlich dargelegt - Selbstverstärkungsmechanismen auf unterschiedlichen Ebenen wirken, so dass sich die Messung von Economies of Scale an wirtschaftlichen Größen wie Stückzahlen und Kostenstrukturen durchführen lässt, Lerneffekte oder Erwartungserwartungen hingegen nicht an numerischen Indikatoren zu erkennen sind. Erschwerend kommt hinzu, dass selbst die Erfassung von Economies of Scale zwar den Nachweis selbstverstärkender Effekte erbringt, die positive Rückkopplung jedoch erst durch die akteursgeleitete Handlung zustande kommt.

Zum zweiten können sich selbstverstärkende Mechanismen nicht nur innerhalb eines Systems bilden, sondern auch in Wechselwirkung mit Elementen der Umwelt entstehen. Die Aufstellung eines allgemeingültigen inhaltlichen Analyserasters zur Identifikation von positiver Rückkopplung ist somit nur schwer möglich. Vielmehr scheint es für die Erhebung unumgänglich, konkreten Indizien und fundierten Vermutungen bezüglich positiver Rückkopplung im jeweiligen Anwendungsfall nachzu- 
gehen. ${ }^{381}$ Grundsätzlich ist darauf zu achten, ob akteursgeleitete Entscheidungen die Attraktivität, erneut in dieselbe Richtung zu entscheiden, erhöhen und sich somit kontinuierliches Entscheidungsverhalten und Attraktivitätssteigerung in einem wechselseitigen Prozess verstärken.

Schließlich beschreibt der Lock-In die vierte und zugleich letzte Komponente strategischer Pfadabhängigkeit. Ein Lock-In untergliedert sich gemäß der in Kapitel 2.5 entwickelten Pfadkonzeption in die Elemente der Ineffizienz, Immunität, Ignoranz sowie der stabilisierenden Faktoren. Ergänzend ist anzufügen, dass sich der Zustand des Lock-Ins als Phase III des Phasenmodells gleichermaßen durch einen gleichförmigen, rigiden Entscheidungsprozess auszeichnet. Strategische Rigidität ist somit ebenfalls ein zentrales Charakteristikum und Element des Lock-Ins, wird aufgrund der ausführlichen Diskussion und Operationalisierung im ersten Schritt der Pfadanalyse dem Rigiditätsnachweis - jedoch nicht erneut angeführt.

Unter Referenz auf die definitorische Abgrenzung von Unternehmen als produktivwirtschaftliche Organisationen ${ }^{382}$ misst sich die (In-)Effizienz einer Organisation als erstes Element strategischer Rigidität an dem Verhältnis zwischen aufgewendeten Mitteln und Zielerreichung. ${ }^{383}$ Das Effizienzniveau eines Unternehmens lässt sich somit als Relation von Kosten als Input und Leistung als Output formulieren, wobei gemäß dem erwerbswirtschaftlichen Prinzip die Erwirtschaftung von Überschuss die oberste Zwecksetzung der Unternehmung ausmacht. Gewinn bzw. Rentabilität stellen somit die relevanten Größen zur Messung von wirtschaftlicher Effizienz dar. Weitere Größen und Kennzahlen zur Erfolgsmessung können sich an der spezifischen zu untersuchenden Unternehmung ausrichten. Im Fall des Bertelsmann Buchclubs als Mitgliedschaftssystem ist eine zentrale Erfolgsgröße die Anzahl der vertraglich gebundenen Mitglieder.

Wie im Methodenteil unter Kapitel 3.5 ausführlich beschrieben, wird auch in dieser Arbeit so vorgegangen.

382 Vgl. hierzu Kapitel 2.1.1 dieser Arbeit.

383 Siehe hierzu das Ökonomische Prinzip oder das Wirtschaftsprinzip, Schmalen, H. (1996), S. 45-52. 
Zur Messung von Immunität ist nach konkreten (gescheiterten) Wandlungsbemühungen zu forschen. Diese können sowohl von außen, z. B. in Form eines Austauschs der Geschäftsführung, an das System herangetragen werden als auch ihren Ursprung innerhalb des Systems haben. Beispiele hierfür sind Neuproduktentwicklungen, der Eintritt in neue Märkte oder Diversifikationsstrategien. Sofern sich diese nicht gegen den dominanten (Strategie)Pfad durchsetzen können und die Bemühungen scheitern, liegt die Vermutung von pfadbedingter Immunität und somit eines Lock-Ins nahe.

Ignoranz kann wie erörtert in drei unterschiedlichen Ausprägungen vorliegen. Die erste Form (,nicht wissen') äußert sich in der mangelnden Kenntnis, einen anderen als den bisher eingeschlagenen Weg gehen zu können. Dies lässt sich beispielsweise an der Ressourcen- und Kompetenzausstattung eines Unternehmens ablesen, das sich auf spezifische Kerngeschäfte spezialisiert und Mittel und Fähigkeiten verloren hat, andere Geschäftsfelder zu bearbeiten. Die zweite Form (,nicht beachten') bezieht sich auf die Blindheit von Organisationen. Getroffene Entscheidungen beruhen auf der fehlenden Reflexionsfähigkeit, weitere Entscheidungsoptionen wahrzunehmen. Ein Indikator für diese Form von Ignoranz ist die dem System unbewusste Ausblendung strategischer Alternativen. Es werden keine weiteren Optionen gesehen. Schließlich äußert sich die dritte Form von Ignoranz (,nicht wissen wollen’) in der bewussten Missachtung von Entscheidungsalternativen, da deren Notwendigkeit nicht wahrgenommen wird. Ein Indikator hierfür ist z. B. die offene Kommunikation oder sogar Befürwortung der strategischen Kontinuität bei gleichzeitiger Ankündigung, den bisherigen Weg weiter beschreiten zu wollen.

Schließlich gilt es, das vierte und zugleich letzte Element eines Lock-Ins in Form von stabilisierenden Faktoren abzubilden. Hierbei ist konkret auf einseitig gerichtete Faktoren zu achten, die zwar den strategischen Entscheidungsverlauf beeinflussen, jedoch von diesem - anders als im Falle der selbstverstärkenden Mechanismen - nicht rückwirkend beeinflusst werden. Mögliche Indikatoren können die Eigentümerstruktur der Organisation, externe Benchmarks, aber auch politische Rahmenbedingungen, die auf die strate- 
gischen Entscheidungsprozesse der Organisation ausstrahlen, sein. Ähnlich wie bereits im Fall der positiven Rückkopplung ist dies nur eine Auswahl an Beispielen, die theoretisch weiterentwickelt oder durch empirische Befunde ergänzt werden können.

Die nachfolgende Tabelle 11 stellt die Operationalisierung der einzelnen Elemente zur empirischen Pfaddiagnose als Schritt 2 in einer Übersicht dar.

Tabelle 11: Operationalisierung der Elemente zur Pfaddiagnose als Schritt 2 der Pfadanalyse

\begin{tabular}{|c|c|c|c|}
\hline & Komponenten & Elemente & Indikatoren \\
\hline \multirow{2}{*}{ Phase I } & \multirow{2}{*}{ Historizität } & Kontingenz & $\begin{array}{l}\text { - Potentielle Entschei- } \\
\text { dungspluralität } \\
\text { - Ergebnisoffenheit }\end{array}$ \\
\hline & & Vorprägung & - Imprints \\
\hline & Critical Juncture & Small Events & $\begin{array}{l}\text { - Wegweisende } \\
\text { Ereignisse }\end{array}$ \\
\hline Phase II & $\begin{array}{c}\text { Positive } \\
\text { Rückkopplung }\end{array}$ & $\begin{array}{l}\text { Selbstverstärkende } \\
\text { Mechanismen }\end{array}$ & $\begin{array}{l}\text { - Economies of } \\
\text { Scale/Scope } \\
\text { - (In)Direkte } \\
\text { Netzexternalitäten } \\
\text { - Lernen } \\
\text { - Erwartungs- } \\
\text { erwartungen } \\
\text { - Koordinationseffekte } \\
\text { - Komplementaritäts- } \\
\text { effekte }\end{array}$ \\
\hline \multirow{5}{*}{ 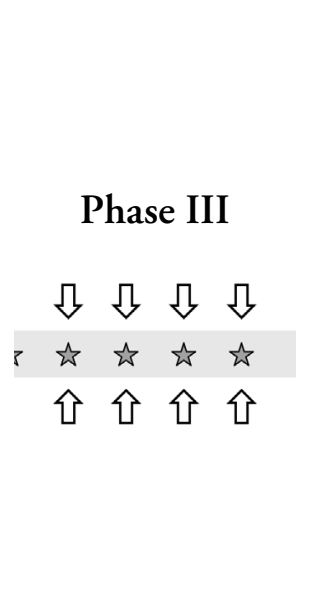 } & \multirow{5}{*}{ Lock-In } & Ineffizienz & $\begin{array}{l}\text { - Mitglieder-/ } \\
\text { Umsatzentwicklung } \\
\text { - Gewinne/Verluste }\end{array}$ \\
\hline & & Immunität & - Wandlungsresistenz \\
\hline & & I & - Unkenntnis \\
\hline & & Ignoranz & - Unwille \\
\hline & & $\begin{array}{l}\text { Stabilisierende Fak- } \\
\text { toren }\end{array}$ & $\begin{array}{l}\text { - Macht } \\
\text { - Eigentümerstruktur } \\
\text { - Benchmarks } \\
\text { - politischer Rahmen }\end{array}$ \\
\hline
\end{tabular}

Quelle: Eigene Darstellung. 
Nachdem die einzelnen Elemente zum Rigiditätsnachweis (Schritt 1) und zur Pfaddiagnose (Schritt 2) operationalisiert worden sind, sollen in den folgenden Kapiteln die Ergebnisse der empirischen Analyse anhand der strategischen Ausrichtung des Bertelsmann Buchclubs dargestellt werden. Die Analyse lehnt sich dabei an die zweischrittige Vorgehenswiese der vorgestellten Konzeption an. In Schritt 1 wird der Entscheidungsverlauf des Bertelsmann Buchclubs auf strategische Rigidität hin untersucht. Liegt ein Rigiditätsbefund vor, gilt es in Schritt 2 festzustellen, ob dieser durch einen pfadabhängigen Prozess aus Historizität, Critical Juncture, positiver Rückkopplung und einem Lock-In zu erklären ist.

\subsubsection{Schritt 1: Rigiditätsnachweis}

Das Konstrukt der strategischen Rigidität setzt sich aus den beiden Elementen der strategischen Kontinuität und dynamischen Umwelt zusammen. Die empirischen Untersuchungsergebnisse zu beiden Elementen werden nun ausführlich erörtert.

\subsubsection{Strategische Kontinuität}

Wie unter Kapitel 2.2.2 dargestellt, basiert der Strategiebegriff in der vorliegenden Arbeit auf drei grundlegenden Dimensionen: dem ,Geschäftsmodell' als zentrale Geschäftsidee, der ,Corporate Strategy’ als Geschäftsfeldstrategie sowie der ,Business Strategy' als konkrete Wettbewerbsstrategie.

Diese drei Ebenen wurden über den Analysezeitraum von der Gründung des Clubs 1950 bis zum Jahr 2007 untersucht, beginnend mit dem Geschäftsmodell.

\subsection{Geschäftsmodell}

Um die Ausgestaltung des Geschäftsmodells im Zeitverlauf vergleichen zu können, wurden zunächst die zentralen Säulen der Geschäftsidee des Buchclubs zum Zeitpunkt der Gründung 1950 erhoben, um diese einem inhaltlichen Vergleich über den Analysezeitraum bis zum Jahr 2007 zu unterziehen: 
Zur Gründung 1950 wurden im Bertelsmann Lesering „mit einem zunächst nur 62 Titel umfassenden Programm [...] Bücher im Direktvertrieb als Abonnement zu reduzierten Preisen vertrieben. “384 Der Monatsbeitrag von 3,20 DM („oder in Vierteljahresraten von 9,60 DM“) berechtigte die Mitglieder des Leserings, „aus der gesamten schöngeistigen Produktion des Verlages Bertelsmann [...] jährlich 8 Bände ohne Rücksicht auf den Ladenpreis aus[zu]wählen.“ Weiterhin erhielten die Mitglieder einen regelmäßig erscheinenden Angebotskatalog, in welchem [Bertelsmann] die beiden Hauptvorschlagsbände ganz besonders heraus[stellte].“" ${ }^{385}$

Die Gründungsidee des Leserings basierte somit auf einem mitgliedsgebundenen Abonnentensystem, das die Clubkunden zum regelmäßigen Kauf verpflichtete. Mitglieder wurden durch einen zweistufigen Vertriebsmodus geworben und betreut: Externe Vertriebsfirmen des Reise- und Versandbuchhandels, des Werbenden Buch- und Zeitschriftenhandels sowie des stationären Sortimentshandels akquirierten die Mitglieder des Clubs und blieben rechtliche Eigentümer der Adressbestände. Bestellungen wurden über einen regelmäßig erscheinenden Clubkatalog getätigt und von Bertelsmann auf postalischem Weg ohne Zwischenschaltung des stationären Buchhandels direkt an die Kunden versandt. Das Programmangebot enthielt eine im Vergleich zur Gesamttitelproduktion auf dem deutschen Buchmarkt stark limitierte Titelanzahl von Büchern, die sich als Lizenzausgaben in Aufmachung und Ausstattung von den Originalauflagen unterschieden. So war es dem Lesering als Buchgemeinschaft rechtlich möglich, unter Umgehung des gebundenen Ladenpreises seine Bücher preisgünstiger anzubieten.

\footnotetext{
384 Derclub.de (2008a).

385 BUA-0039/1 (Lesering: Gründung, 1950); vgl. Gööck, R (1968), S. 116-118.
} 
Zusammenfassend bildeten fünf Säulen die grundlegenden strategischen Pfeiler des Bertelsmann Buchclubs im Jahr 1950:

- Mitgliedschaftssystem,

- Zweistufigkeit,

- Direktvertrieb,

- Selektives Programm,

- Handel mit vergünstigten Buchlizenzen.

Ein inhaltlicher Vergleich dieser fünf zentralen Säulen des Geschäftsmodells führte zu folgenden Ergebnissen:

\section{Mitgliedschaftssystem}

Das bestehende Mitgliedschaftssystem als Buchverkauf im Abonnement wurde grundsätzlich beibehalten, die konkrete Ausgestaltung der Kundenverpflichtung jedoch mehrfach modifiziert.

Die Höhe der Mitgliedsbeiträge wechselte häufig, erstmals wurden diese bereits ein Jahr nach der Lesering-Gründung angehoben. ${ }^{386}$ Die genauen Beitragsmodalitäten unterlagen ebenfalls einer regelmäßigen Überarbeitung. Im Jahr 1951 wurde z. B. ein Punktesystem eingeführt, nach dem die Mitglieder ihre Buchbestellungen auf Basis eines Punktekontos tätigen konnten. ${ }^{387}$ Selbiges wurde zu Beginn der 70er Jahre wieder abgeschafft. 1969 änderte sich die ,Beitragsverpflichtung' zu einer ,Abnahmepflicht'. Allerdings bestand die „wichtigste Änderung [...] darin, dass an die Stelle der ,Beitrags-Verpflichtung' der Begriff ,Abnahmepflicht', und zwar in Höhe der bisher geltenden Beiträge getreten ist. [...] Die neuen ,Mitgliedsvereinbarungen’ unterschei-

386 Vgl. Gööck, R. (1968), S. $124 \mathrm{f}$.

387 Ein Punkt entsprach einem Geldwert von DM 1,90. Jedes Mitglied musste Bücher im Wert von sechs Punkten pro Quartal tätigen. Vgl. Gööck, R. (1968), S. $126 f$. 
den sich rein sachlich kaum von den bisherigen Bedingungen. “388 Statt eines turnusmäßigen Beitrages waren die Mitglieder fortan verpflichtet, aus dem Angebot des Leserings Bestellungen im Mindestwert von DM 15,- pro Quartal zu tätigen. ${ }^{389}$ Es handelte sich somit lediglich um eine rein begriffliche Änderung zur kundenfreundlichen Darstellung, ohne den inhaltlichen Kern der Mitgliedschaftsverpflichtung wesentlich zu beschneiden.

Auch die Konditionen der Abnahmepflicht wurden im weiteren Zeitverlauf - parallel zur Erweiterung des Buchclubprogramms um Non-Book-Artikel - mehrfach modifiziert. Eine wesentliche Änderung erfolgte in den 90er Jahren durch die Aufhebung der quartalsmäßigen Verpflichtung zum Buchkauf hin zu einem beliebigen Artikel aus dem Angebotsprogramm des Clubs. Diese Lockerung der Mitgliedschaftsbedingungen wurde wieder aufgehoben; im Jahr 2007 verpflichteten sich die Mitglieder des Clubs wieder zur Abnahme eines Buchs pro Quartal. ${ }^{390}$

Festzuhalten ist, dass die inhaltliche Ausgestaltung der Mitgliedschaftsverpflichtung im Verlauf der Clubhistorie mehrfach modifiziert, der Kern des festen Abonnements zur turnusmäßigen Kaufverpflichtung jedoch kontinuierlich beibehalten wurde. ${ }^{391}$

\section{Zweistufigkeit}

Auch die zweite Säule des Geschäftsmodells - das zweistufige Buchgemeinschaftssystem - zeichnete sich durch ein hohes Maß an Konstanz über den Analysezeitraum von 1950 bis 2007 aus. Zentrale Gründungsidee war, „die Werbe- und Betreuungsarbeit zu dezentralisieren, auf die Buchhandlungen aller Sparten zu verlagern. “" ${ }^{392}$ So wurden

BUA-UP2/129-1969 (Rundschreiben, 1969), S. 3.

Vgl. BUA-0006/30 (Lesering: Vertrag, 1970).

Vgl. Derclub.de (2008b).

Ergänzend ist anzuführen, dass die Aufhebung der Mitgliedschaftsverpflichtung mehrfach kritisch diskutiert wurde und auch Tests eines mitgliedschaftsfreien Buchvertriebs durchgeführt wurden. Dennoch hat sich die Aufhebung der Kaufverpflichtung nicht durchgesetzt.

Gööck, R. (1969), S. 122. 
Neumitglieder nicht von Bertelsmann selbst, sondern von externen Vertriebsfirmen des vertreibenden Buchhandels geworben und betreut. Als Vergütung leistete Bertelsmann den Vertriebsfirmen Ausgleichszahlungen in Form von Nutzungsentgelten. Durch die stark ansteigenden Mitgliederzahlen erwies sich die Dezentralisierung der Verwaltungs- und Versandarbeit als hinderlich, woraufhin im Jahr 1954 eine erste Modifikation der Zweistufigkeit vorgenommen wurde:

Mit der Gründung der Verlagsgemeinschaft Bertelsmann Lesering GmbH (VG Rheda) wurde eine zentrale Betreuungsstelle geschaffen, die „im Auftrag der Vertriebsfirmen treuhänderisch alle Verwaltungs- und Betreuungsaufgaben durchführte. “" ${ }^{\text {393 }}$ Die Neuwerbung der Buchclub-Mitglieder lag nach wie vor in der Hand der Vertriebsfirmen, wurde jedoch sukzessive durch eigene Werbemaßnahmen des Buchclubs selbst ergänzt. Eine rechtliche Änderung erfolgte Ende der 80er Jahre durch die Auflösung der Nutzungsentgeltbestimmungen zugunsten eines Pachtvertrags mit den Vertriebsfirmen, die damit die Funktion von (Adress)Verpächtern einnahmen. ${ }^{394}$ Im Gegensatz zu den vorher gültigen Bestimmungen, welche die Vertriebsfirmen am Gewinn des Buchclubs direkt beteiligten und somit zu häufigen Auseinandersetzungen (z. B. über Investitionsentscheidungen) führten, regelte der Pachtvertrag die Zahlung von Umatzprovisionen. ${ }^{395}$ Investitionsentscheidungen konnten so zwar selbständig vom Club getroffen werden, allerdings wurde den Verpächterorganisationen nach wie vor ein hohes Maß an unternehmenspolitischem Mitspracherecht eingeräumt. Der Pachtvertrag besteht bis heute und ist unkündbar. ${ }^{396}$ Kundenadressen. Und wenn ich sage ,halten' ist das so zu verstehen wie Genussscheine. D. h. hier gibt es immer wieder prozentuale Abrechnungen im Quartal, die der Club an die Verpächter umsatzbezogen ausschütten muss." Interview BME-07221 (2007, 18.12.), S. 9. 
Es lässt sich festhalten, dass die Form der zweistufigen Buchgemeinschaft von 1950 bis 2007 im Grundsatz beibehalten wurde. Modifikationen ließen sich bezüglich der vertraglichen Ausgestaltung und der gegenseitigen Aufgabenverteilungen erkennen.

\section{Direktvertrieb}

Die dritte Säule des Buchclub-Geschäftsmodells - der Direktvertrieb - wurde ebenfalls über den Analysezeitraum von 1950 bis 2007 konstant gehalten. Erweiterungen ließen sich in der Anzahl der Vertriebskanäle feststellen.

Zunächst erreichte den Kunden das Programmangebot des Leserings ausschließlich über den Katalog ,Lesering Illustrierte', dem heutigen ,Club-Katalog', der den Mitgliedern im quartalsmäßigen Rhythmus zugesendet wurde und aus dem sie direkt ihre Bestellungen tätigen konnten. ${ }^{397}$ Im Jahr 1964 erfolgte die erste Erweiterung des Katalogverkaufs um einen zusätzlichen Vertriebskanal. Als Bücherstube eröffnete die erste Club-Filiale, deren Netz bis 2007 auf ca. 300 Filialen in ganz Deutschland angewachsen ist. ${ }^{398}$ Hinzu kamen zahlreiche Partner-Club-Filialen. ${ }^{399}$ Die zweite wesentliche Erweiterung erfolgte im Jahr 1999 durch den Aufbau der Online-Präsenz unter ,www.derclub.de' als drittem Vertriebskanal. ${ }^{400}$ Als wesentlicher Kundennutzen wurde bereits zu Gründungszeiten die bequeme Bestellmöglichkeit von Büchern kommuniziert. Dieser Vorteil des Clubmodells wurde gerade angesichts der Erweiterung der Distributionskanäle im Analysezeitraum kontinuierlich hervorgehoben. ${ }^{401}$

Es lässt sich festhalten, dass die direkte Buchdistribution des Clubs an seine Mitglieder beibehalten wurde. Änderungen erfolgten in der Anzahl der Vertriebskanäle.

\footnotetext{
397 Vgl. Gööck, R. (1968), S. 119.

398 Vgl. Derclub.de (2008a).

399 Vgl. WIC (2003/03), S.4.

400 Vgl. Derclub.de (2008a); Derclub.de (2008c).

401 Vgl. Derclub.de (2008d), Interview BME-07101 (2007, 12.03.), S. 2.
} 
Das traditionelle Kataloggeschäft wurde um Filialgeschäfte und Online-Distribution erweitert.

\section{Selektives Programm}

Die vierte grundlegende Säule der Bertelsmann Buchgemeinschaft stützt sich auf die limitierte Angebotsbreite und die damit kommunizierte Auswahlkompetenz des Clubs, auf die ebenfalls bis heute als tragende Säule der Buchgemeinschaft verwiesen wird. Beginnend mit einem Programm von 62 Titeln im Jahr 1950 wurde die Titelanzahl vor allem in den frühen Jahren sukzessive erhöht. 1951 wurden ca. 100 Titel angeboten, 1953 bereits 250, ${ }^{402}$ und 1975 umfasste das Angebotsprogramm des Clubs 737 Titel. ${ }^{403}$ Seit 2006 stehen jährlich ca. 1.000 verschiedene Buchtitel zum Verkauf. Der starke Anstieg der Titelzahlen des Clubprogramms lässt auf eine umfangreiche Angebotserweiterung schließen. Diese muss jedoch relativiert werden, sobald die Entwicklung der gesamten jährlichen Titelproduktionen in Deutschland gegenübergestellt wird. Der relative Anteil der Titelanzahl im Club im Vergleich zur gesamten Titelproduktion in Deutschland schwankt während des Analysezeitraums kontinuierlich zwischen 1,0 und 2,5\%.404 Aufgrund der stetig steigenden Titelproduktion auf dem deutschen Buchmarkt ist sogar ein sinkender relativer Anteil im Buchclubprogramm zu verzeichnen.

Das selektive Programmangebot der im Club vertriebenen Bücher kann also ebenfalls als konstanter Faktor in der Clubhistorie betrachtet werden. Dieser wird dergestalt

402 Vgl. Gööck, R. (1968), S. 129.

403 Vgl. Hutter, M./Langenbucher, W.R. (1980), S. 30.

404 Vgl. hierzu die Jahrgänge ,Buch und Buchhandel in Zahlen’ 1950-2007 des Börsenvereins des Deutschen Buchhandels e.V. Zusammenfassende Darstellungen der Titelproduktion in Deutschland aus den Jahren 1951-1975 finden sich u. a. im Jahrgang 1976, S. 10. Während beispielsweise 1975 in Deutschland insgesamt (Erstauflage und Neuauflage) 43.649 Buchtitel produziert wurden, umfasste der Jahresbestand des Bertelsmann Leserings 737 Buchtitel. Vgl. Börsenverein des Deutschen Buchhandels e.V. (1976), S. 10; Hutter, M./Langenbucher, W.R. (1980), S. 30. 
kommuniziert, dass angesichts der Vielzahl von jährlich erscheinenden Novitäten auf dem deutschen Buchmarkt die besondere Auswahlkompetenz des Clubs betont wird. ${ }^{405}$

\section{Handel mit vergünstigten Lizenzausgaben}

Der fünfte zentrale Faktor des Clubmodells betrifft die gegenüber dem gebundenen Buchpreis reduzierten Preise der Club-Lizenzausgaben: „Diese Abnahmeverpflichtung erlaubte es den Buchgemeinschaften, die Bücher billiger anzubieten. " ${ }^{406}$ Im Anhang der Beitrittserklärung zum Bertelsmann Lesering aus dem Jahr 1956 heißt es exemplarisch: „Es ist einleuchtend, dass hohe Buchauflagen entsprechend günstige Vorzugspreise ergeben, die dann allen Mitgliedern zugute kommen. [...] Einige Bespiele zeigen Ihnen, wie günstig Sie als Lesering-Mitglied Bücher erwerben: Der Vorzugspreis für Lesering-Mitglieder beträgt beim Bertelsmann-Hausatlas 30,- DM, während der Preis für Nichtmitglieder 39,- DM ausmacht. [...] Diese Preisvorteile genießen Sie natürlich nur, weil Sie sich zur Lesering-Mitgliedschaft und damit zum ,BücherAbonnement' entschlossen haben. “407 Generell wird zu dieser Zeit der Preisabstand zwischen allgemeinem Buchhandel und Club als „recht erheblich“ ${ }^{408}$ bezeichnet, ohne auf konkrete Prozentangaben einzugehen. Diese Tatsache dürfte wohl dem ohnehin recht angespannten Verhältnis zwischen Sortimentshandel und Buchgemeinschaft Rechnung tragen, das durch direkte Preisvergleiche nicht zusätzlich belastet werden sollte. Im Jahr 2007 firmierte der Club mit klaren Vergleichswerten, indem der Preisabstand zum übrigen Handel von durchschnittlich $25 \%$, bei einigen Buchtiteln jedoch bis zu $70 \%$ herausgestellt wird. Seit 2007 besteht zudem eine Preisgarantie, die

405 „[...] so dass darin der wichtigste Punkt ist, den Buchmarkt überschaubar zu machen für den Normalbürger.“ Interview BME-07010 (2007, 12.03.), S. 2.

406 Vgl. Gööck, R. (1968), S. 112.

407 BUA-0041/21 (Lesering: Anhang zur Beitrittserklärung, 1956).

408 BUA-0041/21 (Lesering: Anleitung für die Vertriebsfirmen, 1958). 
den Mitgliedern die Zahlung des Differenzbetrages zusichert, sofern sie im allgemeinen Handel ein dem Clubangebot identisches Buch zu günstigeren Preisen finden. ${ }^{409}$

Zusammenfassend lässt sich festhalten, dass die tragenden Säulen des Geschäftsmodells - Mitgliedschaft, Zweistufigkeit, Direktvertrieb, begrenzte Programmauswahl sowie Preisvorteile durch Lizenzausgaben - seit der Buchclubgründung 1950 bis zum Jahr 2007 in ihren Grundzügen beibehalten wurden. Anpassungen wie die Modifikation der Mitgliedschaftsbedingungen oder die Erweiterung der Distributionskanäle wurden vorgenommen, jedoch ohne den eigentlichen Charakter der zentralen Clubelemente zu verändern.

Ließ sich mit der Analyse des Geschäftsmodells bereits Kontinuität auf der Ebene des strategischen Selbstverständnisses des Buchclubs nachweisen, so ist im Folgenden zu zeigen, ob die strategischen Entscheidungsprozesse auf Gesamtunternehmensebene einer ebensolchen Kontinuität unterliegen. Erhoben wird der zweite Indikator strategischer Kontintuitiät: die Corporate Strategy.

\subsection{Corporate Strategy}

Als nächste Ebene der Unternehmensstrategie wurde die Entwicklung der strategischen Geschäftsfelder des Clubs im Rahmen der Gesamtunternehmensstrategie (Corporate Strategy) analysiert. Als Untersuchungsraster diente die Produkt/Markt-Matrix, die vier verschiedene Strategietypen abbildet: Markt-Durchdringung, ProduktEntwicklung, Markt-Entwicklung sowie Diversifikation. ${ }^{410}$

\section{Markt-Durchdringung}

Die Analyseergebnisse zeigen, dass der Buchclub von 1950 bis zu Beginn der 90er eine klare Strategie der Markt-Durchdringung verfolgte.

\footnotetext{
409 Derclub.de (2008d).

410 Vgl. Ansoff, H.I. (1968), S. 99.
} 
Zentrales Charakteristikum der Leseringarbeit in den 50er Jahren waren die massiven Werbe- und Vertriebsbemühungen zur Mitgliedergenerierung. Die Einschaltung des Reise-und Versandbuchhandels sowie des Werbenden Buch-und Zeitschriftenhandels zur Mitgliederwerbung bewirkten einen inflationären Anstieg in der Neukundengewinnung. Hinzu kam die von Bertelsmann forcierte Werbeidee der Freundschaftswerbung, die bereits ab 1951 erfolgreich als treibende Kraft zur Vergrößerung des Abonnentenkreises eingesetzt wurde. Die von Bertelsmann wiederholt kommunizierte Leistung der Buchclubarbeit zeichnete sich in der Konsequenz dadurch aus, einen großen Teil der Bevölkerung von Nicht-Lesern zu Lesern zu machen. ${ }^{411}$ Neben der Neukundenakquise wurde die Markt-Durchdringung erstmalig Anfang der 60er Jahre durch die Übernahme konkurrierender Buchgemeinschaften umgesetzt. Diese Strategie wurde vor allem in den 80er Jahren konsequent weiterverfolgt. Der Bertelsmann Buchclub konnte so seinen Marktanteil von $66 \%$ aus dem Jahr 1981 auf 97,5 \% im Jahr 1992 ausweiten. Hierzu trugen vor allem die Übernahmen der größten Wettbewerber Europäische Bildungsgemeinschaft 1972, Deutschen Buchgemeinschaft 1989 sowie Deutscher Bücherbundes 1992 bei. $^{412}$

\section{Produkt-Entwicklung}

Neben der expansiven Markt-Durchdringung bemühte sich der Bertelsmann Buchclub bereits früh, durch die Strategie der Produkt-Entwicklung, die Bedürfnisse des bestehenden Marktes mit neuen Produkten zu befriedigen. Hierzu zählt beispielsweise die Angebotserweiterung um einen Musikbereich, der seit 1956 mit Schallplatten, später mit MusiCassetten bzw. CDs einen festen Bestandteil im Buchclubprogramm bildet. Mit der Gründung des Versandhauses Heim \& Buch im Jahr 1955 wurden den Clubmitgliedern auch Möbel wie Bücherregale, Schränke und Lampen angeboten;

\footnotetext{
411 „Vielmehr wird unsere ganze Kraft erforderlich sein, in zähem Ringen die Grenzen des Marktanteils auszuweiten." BI (1964/01-02), S. 4.

412 Vgl. hierzu ausführlich Kapitel 4.3.1 dieser Arbeit.
} 
1964 erfolgte die Gründung des Club-Reisedienstes, über den die Buchclubmitglieder Urlaubsreisen buchen konnten. ${ }^{413}$

Das Angebot des Non-Book-Bereichs wurde in den 70er Jahren weiter verbreitert. 1971 standen erstmals Spiele zum Verkauf, 1974 und 1975 folgten Kameras und kunstgewerbliche Erzeugnisse. Im Jahr 1976 wurde schließlich ein Fotolabor-Service zur Filmentwicklung für Buchclub-Kunden eingerichtet. ${ }^{414} 1983$ startete ein Videocassetten-Verleih-Service in den Ladenketten des Clubs. ${ }^{415}$ Im Jahr 2007 bestand das Angebotsprogramm des Buchclubs aus einer ganzen Reihe von zusätzlichen Produkten und Dienstleistungen. Neben Büchern, Musik, DVDs, Spielen, Computersoftware und Spielekonsolen werden unter der Rubrik ,Trendshop' u. a. Einrichtungs- und Dekorationsartikel angeboten. Zur Rubrik ,Foto’ zählten Kameras und Fotoservices, die Sparte ,Reisen' vermittelte Urlaubsangebote, und zu den Angeboten des ,Club Extra’ zählten u. a. Kredite und Versicherungen, Handys sowie Veranstaltungstickets und Weiterbildungskurse. ${ }^{416}$

Welchen Stellenwert die Bereiche Musik sowie Non-Book im gesamten Angebotsportfolio des Buchclubs eingenommen haben, zeigt ein Längsschnitt-Vergleich der jeweiligen Umsatzanteile. Deutlich wird hier, dass die Umsatzanteile bis Ende der 70er Jahre nahezu konstant gleichmäßig verteilt waren. Der Bereich ,Buch’ trug zu ca. 80 \%, ,Musik' zu ca. 15 \% und ,Sonstiges' (u. a. Möbel und Reisen) zu ca. 5 \% des Club-Gesamtumsatzes bei. ${ }^{417}$ Die klare Schwerpunktsetzung auf das Produkt ,Buch’ mit dem Zusatzgeschäft Musik findet sich neben den Umsatzzahlen auch in der strategischen Zielsetzung des Buchclubs wieder. So wurde es z. B. im Jahr 1968 „,nicht für

413 Der Club betätigte sich nicht als eigener Reiseveranstalter, sondern arbeitete in Kooperation mit bestehenden Touristikunternehmen. Vgl. Interview BM-07410, S. 22.

414 Vgl. BUA-0006/465 (Hennig, 1977).

415 Vgl. Börsenblatt (1983, 08.03.), S. 539.

416 Vgl. Derclub.de (2008e).

417 Vgl. BUA-0006/455 (Sonderobjekte, o. D.); Börsenblatt (1976, 25.06.), S. 952; BUA0006/464 (Zahlenmaterial, 1974), S. 2. 
richtig [angesehen], das Angebot allzu sehr auf andere Dinge auszuweiten, die mit Buch und Platte gar nichts mehr zu tun haben. ““18 Ähnlich im Jahr 1977: „Keinesfalls darf durch diese Sonderprogramme der Umsatz mit dem Kernprodukt 'Bücher' und 'Tonträger' geschmälert werden. Das könnte auch dadurch geschehen, dass eine zu starke Ausweitung der Sonderprogramme das Image der Clubs in dem Sinne verändert, dass sie nicht als vornehmliche Buch- und Musik-Anbieter von den Kunden beziehungsweise den Mitgliedern erkannt werden.“"419

Mit Beginn der 80er Jahre erfolgte eine latente Schwerpunktverschiebung in Richtung des langsam wachsenden Non-Book-Bereichs. ${ }^{420}$ Diese Entwicklung stand jedoch unter kritischer Beobachtung, wurde doch nach wie vor das Kerngeschäft des Clubs im Bereich ,Buch' gesehen: „Schwerpunkt unseres Angebots bleibt in jedem Falle das Buch, nicht nur aus traditionellen, auch aus wirtschaftlichen Gründen. “" ${ }^{421}$ Diese Aussage bestätigte sich ebenfalls im Jahr 1988, indem in einer umfassenden Geschäftskonzeption die Fokussierung auf Medieninhalte ('Bücher, Audioträger und Videoträger' mit besonderem Gewicht auf dem Bereich Buch) konstatiert wurde. ${ }^{422}$ Zusatzangebote konnten dieses Programm aus kalkulatorischen Gründen sowie zur Steigerung der Mitgliedschaftsattraktivität ergänzen, allerdings sollten sie folgenden Kriterien gerecht werden: „Verträglichkeit mit dem Kernprogramm“, „keine Dominanz der Zusatzangebote im Umsatzanteil und im äußeren Erscheinungsbild (Katalog, Läden)“ sowie eine „strenge Unterordnung unter die allgemeinen Grundsätze der Geschäftspolitik und der Marktpositionierung“ “423 Sämtliche Zusatzgeschäfte wurden am Nutzen für das Kerngeschäft gemessen.

\footnotetext{
418 BUA-0006/75(2) (Beiratsprotokoll, 1968, 04.11.), S. 7.

419 BUA-0006/272 (Programmbeirat, 1977), S. 29.

420 Vgl. FAZ (1983, 22.11.); Die Welt (1983, 22.11.).

421 Börsenblatt (1983, 31.03.), S. 817.

422 Vgl. Preußner, N. (1989), S. $232 \mathrm{f}$.

423 Preußner, N. (1989), S. 238.
} 
Trotz des erklärten Ziels der Markt-Durchdringung im Buchbereich erfolgte bis Mitte der 90er eine stetige Umsatzausweitung des Musik- und Non-Book-Bereichs: 1992 entfielen nur noch 55 \% des Umsatzes auf den Buchsektor, wohingegen 28 \% aus dem Bereich ,Musik' und $17 \%$ aus dem Bereich ,Sonstiges' stammten. ${ }^{424} 1994$ sank der Anteil des Buchumsatzes im Club erstmals unter $50 \%$, so dass die Buchgemeinschaft den Großteil ihres Umsatzes im Nicht-Buch-Bereich erwirtschaftete. ${ }^{425}$ Dieser beschriebenen Tendenz der programmatischen Ausweitung über die Grenzen des Buch- bzw. Medienvertriebs hinaus wurde Ende der 90er Jahre mit einer konsequenten Refokussierung begegnet. Das Produkt Buch rückte wieder als Kerngeschäftsfeld in den strategischen Mittelpunkt: „Deshalb wollen wir uns wieder deutlicher auf Medienprodukte konzentrieren und vor allem den Buchanteil stärken. ““26 Dieser Anspruch, das Buch „wieder an die erste Stelle im Programmmix des Clubs“ ${ }^{427}$ zu rücken, war bis 2007 gültig und wird nach wie vor als die zentrale Geschäftsfeldstrategie betrachtet. Ergänzend soll die „mediale Vielfalt“ ${ }^{“ 28}$ der übrigen Angebote die Attraktivität und Kundenfreundlichkeit des Buchclubs stärken.

424 Vgl. Kollmannsberger, M. (1995), S. 47. Ebenso: „In Deutschland hat man irgendwann in den 80er Jahren, 90er Jahren gesagt, wir müssen weg vom reinen Buchclub und müssen zum Medienclub, weil der Buchmarkt nicht so stark wächst. Es kamen neue Medien auf, es kam die $\mathrm{CD}$, es kam die DVD und Video und so weiter, und dann ist man sehr stark in diese neuen Medien eingestiegen und hat diesen Bedarf auch befriedigt..." Interview BME-07021, S. 13. Vgl. Kollmannsberger, M. (1995), S. 47. Parallel zu den sinkenden Buchumsätzen erstarkten andere Programmsparten: „Im Windschatten von Buch und Musik segelt unauffällig aber erfolgreich der Programmbereich Spiele/Spielzeug. Mit zweistelligen Millionenumsätzen ist er aus dem Club-Programm nicht mehr wegzudenken.“ WIC (1991/02), S.5; „Langsam aber sicher wächst sich unser Club-Foto-Dienst zu einem Angebotszweig aus, den unsere Mitglieder nicht mehr missen wollen.“ Vgl. WIC 1991/04), S. 3; „Der Club könnte ohne Bücher nicht leben, aber auch die Musik ist unverzichtbarer Bestandteil des Programms." Vgl. WIC (1995/11).

427 WIC $(2005 / 02)$, S. 5.

428 Gohr, S. (2006), S. 32. 


\section{Markt-Entwicklung}

Neben Markt-Durchdringung und Produkt-Entwicklung wurden im Verlauf der Buchclub-Historie auch wiederholt Bemühungen zur Markt-Entwicklung unternommen. Wie bereits in der unternehmenshistorischen Darstellung unter Kapitel 4.3.1.2 erläutert, expandierte der Club erstmals 1962 durch die Gründung des spanischen Buchclubs Círculo de Lectores in ausländische Märkte. Es folgten eine Vielzahl von weiteren Gründungen ausländischer Bertelsmann Buchgemeinschaften, zuletzt in Osteuropa und Asien. Die ausländischen Clubs wurden weitestgehend nach deutschem Vorbild geführt, orientierten sich jedoch an den jeweiligen nationalen Marktbedingungen. In diesem Sinne arbeiteten die einzelnen Clubs programmatisch unabhängig. ${ }^{429}$ Die Organisationsform der ausländischen Bertelsmann Buchclubs als weitgehend autonom agierende Profit Center entrückt die Geschäftstätigkeiten der ausländischen Buchclubs von der strategischen Entwicklung des deutschen Buchclubs. ${ }^{430}$ Zwar werden die einzelnen Clubs in regionale Bereiche gebündelt (so z. B. die deutschsprachigen Clubs mit England, Spanien und Portugal) ${ }^{431}$, allerdings als strategisch und operativ eigenständig wirtschaftende Unternehmen geführt. ${ }^{432}$ In diesem Sinne können die ausländischen Buchclubs auch nur bedingt einer MarktEntwicklungsstrategie des deutschen Clubs zugeordnet werden, da die Strategie der Markt-Entwicklung eher auf der Ebene der DirectGroup anzusiedeln ist.

Im Gegensatz dazu wurden auf der Ebene des deutschen Clubgeschäfts durch die Integration von $B O L$ bzw. die Gründung der Bereiche Best!Seller sowie Boulevard neue Marktsegmente erschlossen. ${ }^{433}$ Best!Seller, gegründet 1996, vertrieb als freier Medien-

\footnotetext{
429 Vgl. Lehning, T. (2004), S. 54.

430 Vgl. Bertelsmann.de (2008a).

431 Vgl. Directgroup-bertelsmann.de (2008).

432 In diesem Sinne werden die ausländischen Buchclubs in dieser Arbeit als Systeme der verwandten Umwelt und somit als relevante Kontextfaktoren des Clubs angesehen.

433 Vgl. hierzu Kapitel 4.3.1.4 Arbeit.
} 
versender schwerpunktmäßig Bücher, Musik und Videos und versendete analog zum Buchclubmodell einen Produktkatalog. Neu war, dass keine Abonnementen geworben wurden, so dass der Club mit Best!Seller erstmalig in das Segment des nicht mitgliedschaftsgebundenen Medienvertriebs eindrang. Mit der ebenfalls 1996 gegründeten Buchhandelskette Boulevard positionierte sich der Club zusätzlich im Marktsegment des Bucheinzelhandels und erweiterte somit sein traditionelles Produktportfolio um eine neue Vertriebsform. 2001 wurde $B O L$ schließlich als Online-Plattform zum Medienvertrieb in den Clubbereich integriert. Auch hier besetzte der Club durch die ECommerce-Aktivitäten mit Buch-, CD-, Video- und Unterhaltungsprogramm den bislang neuen Bereich des elektronischen Handels. Wie bereits unter Kapitel 4.3.1.4 erwähnt, folgte auf die expansive Geschäftsstrategie wenige Jahre später der Rückzug auf das angestammte Geschäftsfeld des Buchclubs: die Refokussierung auf das traditionelle Buchclubmodell.

\section{Diversifikation}

Die vierte Strategieform - Diversifikation - lässt sich weniger im Club selbst, sondern eher auf der Ebene des Bertelsmann Konzerns feststellen. Dieser ist mit seinen Divisionen in den Bereichen Medienclubs, Druck und Dienstleistungen, Buchverlage, Zeitschriften, Fernsehen und Hörfunk sowie Musikvermarktung in einer Vielzahl von Medienmärkten vertreten. ${ }^{434}$ Der Ursprung der heutigen Konzernentwicklung ist in der Tat auf die ,Keimzelle’ des deutschen Buchclubgeschäfts zurückzuführen, da - wie in Kapitel 4.3.1.2 erörtert - mit der Gründung des Leserings die Geschäftsaktivitäten des C. Bertelsmann Verlags im Jahr 1950 neu aufgebaut wurden. So lässt sich die Random House-Gruppe auf das Verlagshaus C. Bertelsmann zurückführen, die Musikvermarktung BMG auf die Produktionsfirma Ariola, die Druckereien von Arvato auf die Technik von Mohn \& Co. GmbH, die Verbindung zum Film- und Fernsehgeschäft 
$(R T L)$ lässt sich über den Einstieg bei der $U F A$ herstellen. Auch wenn bereits erste Versuche im Zeitschriftenmarkt bestanden, dürfte lediglich der Erwerb von Gruner+Jahr nicht auf direktem Wege aus der Unternehmensgeschichte des Bertelsmann Konzerns abgeleitet werden können. ${ }^{435}$

Im Ergebnis lässt sich festhalten, dass das Medienhaus Bertelsmann mit unterschiedlichen Produkten auf unterschiedlichen (Medien-)Märkten tätig und somit als Medienunternehmen stark diversifiziert ist. Diese Entwicklungen sind jedoch nicht auf strategischer Ebene des (deutschen) Buchclubs zu sehen. In der Entwicklung der Buch-, Schallplatten- bzw. Medienclubs ließen sich keine Diversifikationsstrategien feststellen.

Abbildung 12: Übersicht der Corporate Strategy anhand der Produkt/Markt-Matrix

\begin{tabular}{|c|c|c|c|c|c|}
\hline & \multicolumn{4}{|c|}{ Produkt } \\
\hline & & alt & & neu & \\
\hline \multirow{2}{*}{ 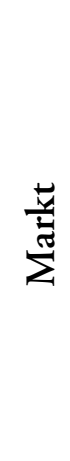 } & न & $\begin{array}{l}\text { Markt-Durchdringung } \\
\text { Marktanteil ,deutsche } \\
\text { Buchgemeinschaften': }\end{array}$ & $97,5 \%$ & $\begin{array}{l}\text { Produkt-Entwicklung } \\
\text { Schwerpunkt Buch: } \\
\text { Ausweitung Non-Book: } \\
\text { Refokussierung Buch: }\end{array}$ & $\begin{array}{l}1950-1980 \\
1980-2000 \\
2000-2007\end{array}$ \\
\hline & $\stackrel{\Xi}{\Xi}$ & $\begin{array}{l}\text { Markt-Entwicklung } \\
\text { Best!Seller: } \\
\text { Boulevard: } \\
\text { BOL: }\end{array}$ & $\begin{array}{l}1996-1999 \\
1998-1999 \\
1999-2002\end{array}$ & Diversifikation & \\
\hline
\end{tabular}

Quelle: Eigene Darstellung in Anlehnung an Ansoff, H.I. (1968), S. 99.

Abbildung 12 fasst die wesentlichen Strategieentwicklungen des deutschen Buchclubs auf Geschäftsfeldebene anhand der beschriebenen Produkt-/Markt-Matrix graphisch

435 Ergänzend ist anzuführen, dass Mitte der 60er Jahre der Entschluss fiel, in die industrielle Landwirtschaft einzusteigen. Es entstand die von Bertelsmann betriebene Hühnerfarm „Hennengold' mit mehr als einer Million Legehennen. Eine Änderung der EWGFuttermittelverordnung sowie die Rückbesinnung von Bertelsmann auf Medienmärkte führten im Jahr 1971/72 zur Aufgabe der Farm. Vgl. Bertelsmann.de (2008e). 
zusammen. Wichtig ist zu betonen, dass sich die Strategieentwicklung auf die Ebene des deutschen Buchclubs und nicht auf die Gesamtunternehmensebene bezieht.

Nachdem bereits über weite Phasen hinweg kontinuierliche Entscheidungsverläufe auf den Ebenen des Geschäftsmodells sowie der Corporate Strategy nachgewiesen werden konnten, soll die nun folgende Analyse der Business Strategy die empirische Untersuchung zum kontinuierlichen Strategieverlauf im Bertelsmann Buchclub vervollständigen.

\subsection{Business Strategy}

Die Analyse der Wettbewerbsstrategie des deutschen Bertelsmann Buchclubs konzentrierte sich auf drei zentrale Bereiche: den Ort des Wettbewerbs, die Regeln des Wettbewerbs sowie den Schwerpunkt des Wettbewerbs. ${ }^{436}$

\section{Wettbewerbsort}

Beginnend mit dem Wettbewerbsort ließ sich kontinuierlich seit der Gründung das Bemühen des Clubs feststellen, den gesamten Markt abzudecken. Analog zu dem von Bertelsmann erhobenen Anspruch „Bücher für Millionen““37 zu vertreiben, erfolgte keine Einschränkung auf spezifische Kundensegmente. Im Gegenteil: Zu der für den Buchhandel klassischen Zielgruppe der bibliophilen Bevölkerung wurde gezielt versucht, auch weniger gebildete Schichten anzusprechen und für das Produkt ,Buch’ zu motivieren. Bis heute wird diese Strategie der Gesamtmarktabdeckung kontinuierlich verfolgt, nicht zuletzt durch die breite Programmaufstellung verschiedener Buchtypologien wie Belletristik, Sach- und Kinderbücher. ${ }^{438}$ Die einzelnen Filialen der stationä-

\footnotetext{
436 Vgl. Steinmann, H./Schreyögg, G. (2005), S. 221.

437 Gööck, R. (1968).

438 „Im Laufe all der Jahre hat sich in der soziologischen Struktur unserer Mitglieder nichts geändert, sie deckt sich mit der Bevölkerung. Unter soziologischen Gesichtspunkten haben wir also keine neuen Märkte erschließen können. Allerdings findet sich eine leichte Tendenz zu sozial höher gestellten Bevölkerungsschichten. Wollten wir uns um diese Kreise bemühen, müssten
} 
ren Ladenkette erschließen sich über ganz Deutschland, regionale Schwerpunktsetzungen ließen sich im gesamten Analysezeitraum nicht feststellen.

Mit der Einführung von Spezialclubs wurde seit Mitte der 80er Jahre immer wieder versucht, parallel zur breiten Marktabdeckung auch spezielle Kundengruppen anzusprechen und sich so in Nischenmärkten zu positionieren. Der Spezialclub Moments richtete sich beispielsweise vor allem an Frauen mit Interesse an gefühlvoller Unterhaltungsliteratur. Weitere Spezialclubs waren geplant, wurden jedoch - ebenso wie $M o$ ments - nicht weiter geführt. ${ }^{439}$

\section{Wettbewerbsregeln}

Mit der Gründung des Leserings gelang es Bertelsmann, die bisherigen Wettbewerbsregeln - der zweite zu untersuchende Gegenstand der Business Strategy - neu zu definieren und somit die Rolle eines ,Rule Breakers' einzunehmen. Wie im Rahmen der Umweltanalyse in Kapitel 4.4.2.2 noch ausführlich erörtert werden wird, bestanden zum Gründungszeitpunkt des Leserings sowohl bereits andere Buchgemeinschafen als auch Bucheinzelhandelsgeschäfte. Der Bertelsmann Buchclub setzte sich allerdings mit seiner zweistufigen Vertriebsidee über die bisherige Trennung von Bucheinzelhandel und Buchgemeinschaften hinweg und suchte die Kooperation mit dem vertreibenden Buch(einzel)handel. Vor allem durch die Zusammenarbeit mit dem Reise- und Versandbuchhandel sowie dem Werbenden Buch- und Zeitschriftenhandel schaffte es Bertelsmann, eine große Vertriebsmacht zu generieren, die sich innerhalb von wenigen Jahren in exponentiell steigenden Mitgliederzahlen als Buchkäufer äußerte. Statt wie bisher den ,Kunden zum Buch zu bringen’ kehrte der Buchclub Bertelsmann die etab-

wir auch ein anderes Programm machen, doch das ist nicht unsere Aufgabe. Wir sind nun einmal angetreten, etwas für die breite Masse zu tun.“ Börsenblatt (1975, 12.09.), S. 1199. „Mit unserem Programmangebot versuchen wir möglichst große Teile der Bevölkerung abzudecken.“ Preußner, N. (1989), S. 232.

439 „Das deutsche Clubgeschäft hat nach bisherigen Erfahrungen beschlossen, dass Spezialclubs künftig keine Priorität in der Geschäftpolitik haben. "Preußner, N. (1989), S. 245. 
lierte Regel um. Es hieß: „Nicht auf den Leser warten. Das Buch zum Leser tragen, den Kunden zum Lesen erziehen. ${ }^{{ }^{440}}$ Aus der bereits festgestellten strategischen Kontinuität auf den Ebenen des Geschäftsmodells und der Corporate Strategy sowie der Tatsache, dass auch neu gesetzte Wettbewerbsregeln in dynamischen Märkten veralten können, ${ }^{441}$ lässt sich ableiten, dass die von Bertelsmann initiierte Neudefinition des Buchmarktes ihren Status als Rule Breaker im Laufe der 57-jährigen Unternehmenshistorie eingebüßt hat. ${ }^{442}$

\section{Strategische Stoßrichtung}

Bei der Ermittlung der dritten zentralen Säule der Business Strategy, der strategischen Stoßrichtung, mit welcher den Wettbewerbern gegenübergetreten wurde, ist die Dominanz der Kostenschwerpunkt-Strategie vor allem in den Jahren von 1950 bis 1980 immanent. Die Kostenstruktur der Buchherstellung beruht auf einem hohen Fixkostenanteil, so dass mit steigenden Auflagenzahlen sinkende Stückkosten erzielt werden können. ${ }^{443}$ Zur Ausnutzung dieser Kostendegressionseffekte war das Zusammenspiel einer ganzen Reihe von Faktoren des Clubmodells zuträglich. Die quartalsmäßige Kaufverpflichtung der Clubmitglieder konzentrierte sich auf das in der Auswahl stark limitierte und populäre Buchangebot im Club. In der Folge erzielten viele Titel Auflagenhöhen, welche weit über dem Buchhandelsdurchschnitt lagen. Aufgrund der möglichen preislichen Unterschreitung des gebundenen Ladenpreises konnte der Club die geringen Stückkosten der Buchherstellung in Form von relativen Preisvorteilen an seine Mitglieder weitergeben. Wie im Rahmen der Analyse des Geschäftsmodells bereits dargestellt, wirbt der Club seit seiner Gründung bis heute mit günstigeren Preisen ge-

\footnotetext{
440 Wirtschaftswoche $(1985,14.06$.$) .$

441 Vgl. Steinmann, H./Schreyögg, G. (2005), S. 223.

442 „Hier fehlt einfach einen andere, einfache, zündende Idee“, Interview BE-07033 (2007, 17.07.), S. 3. „Das Gesetz des Handelns hat der Club über viele Jahre bestimmt. Und das haben inzwischen jetzt andere übernommen." Interview BE-07033 (2007, 17.07.), S. 10.

443 Vgl. Schumann, M./Hess, T. (2006), S. 41-43.
} 
genüber dem regulären Buchhandelspreis und stellt so seine preislichen Vorzüge gesondert heraus.

Zunehmender Wettbewerb sowie ansteigende Kosten des Vertriebssystems bei gleichzeitig sinkenden Auflagenzahlen erwirkten ab den 80er Jahren eine stärkere Qualitätsorientierung: „Der Grundstein für die zukünftige Club-Politik ist bereits gelegt worden: Beim diesjährigen Treffen aller Führungskräfte des deutschen Clubgeschäfts [...] war die Zielsetzung Qualität das bestimmende Leitthema." "444 Besonders hervorgehoben wurden literarische Kompetenz, Auswahl- und Beratungsleistung sowie die innovative Programmpolitik des Clubs. ${ }^{445}$ In der Außendarstellung äußerte sich die Neupositionierung in weit reichenden Renovierungsarbeiten der Filialgeschäfte sowie einem Relaunch der Website. ${ }^{446}$ Zudem wurde verstärkt auf Aktualität und Exklusivität in der Programmauswahl gesetzt; erstmalig ab 1993 erschienen sog. Club Premieren exklusiv im Club, ohne vorher im klassischen Sortimentshandel erhältlich gewesen zu sein. ${ }^{447}$

Trotz des gestiegenen Qualitätsbewusstseins im Clubgeschäft wird nach wie vor auf die preislichen Vorteile der Clubprodukte hingewiesen und mit Preisgarantien geworben. ${ }^{448}$ Erklärtes Ziel ist es heute, nicht mit den in den Markt eingetretenen Billiganbietern zu konkurrieren, sondern sich durch ein verbessertes Programm- und Servi-

444 WIC (1988/08), S. 2; Der Zielkonflikt zwischen besonderen Serviceleistungen und der kostengünstigen Abwicklung eines Massengeschäfts wurde problematisiert: „Die Rahmenvorstellungen konkurrieren in den Zielen wenigstens teilweise miteinander." Preußner, N. (1989), S. 234.

445 Vgl. BG (1987/88), S. 14.

446 Vgl. WIC (2003/07), S. 2, Börsenblatt (2003/38), S. 21.

447 Vgl. WIC (1998/02), S. 4. Im Jahr 1998 wurde erstmals ein internationaler Bestseller als ClubPremiere eingeführt. Die Ausweitung der Club Premieren auf internationale Bestseller verlief nicht ohne Widerstand auf Seiten des Sortimentshandels.

448 Noch 1999 wird von der „Kernkompetenz“, dem „Preisvorteil“ gesprochen. Börsenblatt (1999, 16.11.), S. 5. 
ceangebot bei gleichzeitig attraktiven Preisen von den Wettbewerbern zu differenzieren. ${ }^{449}$

\subsection{Zwischenfazit zu strategischer Kontinuität}

Zusammenfassend lässt sich feststellen, dass die einzelnen Ebenen der strategischen Entwicklung des Clubs über den Analysezeitraum von 1950 bis 2007 ein hohes Maß an Konstanz aufwiesen.

Die zentralen Säulen des Geschäftsmodells - Abonnentensystem, Zweistufigkeit, Direktvertrieb, begrenzte Programmauswahl sowie der relative Preisvorteil durch Lizenzausgaben - besaßen über den gesamten Analysezeitraum Gültigkeit. Die vorgenommenen Änderungen wie die Art der Mitgliedschaftsverpflichtung oder die Erweiterung der Distributionskanäle änderten nichts an der grundsätzlichen Konstanz der strategischen Geschäftssäulen.

Bezüglich der Corporate Strategy des Clubs ließ sich eine eindeutige Dominanz der Markt-Durchdringung feststellen, deren Ergebnis sich in einem Marktanteil von 97,5\% des Bertelsmann Clubs manifestierte. Produkt-Entwicklungen wurden bereits wenige Jahre nach der Buchclubgründung durch Programmerweiterungen um einen Musik- und Non-Book-Bereich vorgenommen. Beide Sparten liefen jedoch vornehmlich als ergänzende Zusatzgeschäfte, um das Kerngeschäftsfeld des Buchvertriebs abzusichern und zu unterstützen. Anfang der 90er erfolgte eine starke Erweiterung der $\mathrm{Zu}-$ satzgeschäfte, so dass erstmals mehr als die Hälfte des Buchclub-Umsatzes nicht mit Büchern erwirtschaftet wurde. Diesem Trend wurde wenige Jahre später mit einer klaren Refokussierung auf das Buch widersprochen. Ab Mitte der 60er Jahre wurden

449 „Billiger zu sein als andere alleine reicht als Argument für den Beitritt zum Club nicht mehr aus. Von den Mitgliederbefragungen wissen wir: Unserer Kunden wollen beraten werden und sie erwarten qualifizierte Empfehlungen. Das bedeutet nicht nur ein Abbilden des Marktes, sondern es muss darüber hinaus gehen: In der Art, wie wir angebotene Titel empfehlen, in der Qualität der Produkte und der Exklusivität der Bücher muss sich das unverwechselbare Profil des Clubs zeigen." WIC (2005/02), S. 2. 
neue geographische Märkte durch Expansion ins Ausland erschlossen. Die entstandenen internationalen Buchgemeinschaften wurden jedoch nicht als Tochterunternehmen des deutschen Clubs gegründet, sondern als organisatorisch selbständige Einheiten innerhalb des Unternehmens Bertelsmann geführt. Aus diesem Grund lässt sich nicht von einer Markt-Erweiterungs-Strategie des deutschen Buchclubs sprechen, sondern die strategischen Entwicklungen sind auf der übergeordneten Divisions- bzw. Konzernebene einzuordnen. Markt-Entwicklungen auf Clubebene wurden erst in den 90er Jahren auf dem deutschen Markt angestoßen (Best'Seller, Boulevard, BOL), wenige Jahre später jedoch wieder eingestellt. Diversifikationsstrategien ließen sich zwar auf Konzernebene feststellen, innerhalb des deutschen Buchclubs jedoch nicht.

Die Analyse der Business Strategy führte zu folgenden Resultaten: Der Club verfolgte kontinuierlich die Strategie der Gesamtmarkt-Abdeckung. In den 80er Jahren wurde durch die Gründung von Spezialclubs mehrfach versucht, Marktnischen zu bedienen. Diese Versuche wurden auf dem deutschen Markt jedoch wieder eingestellt. Durch die Kooperation mit dem vertreibenden Buchhandel und die Entwicklung des zweistufigen Vertriebsmodells definierte der Bertelsmann Lesering zur Geschäftsgründung die Regeln des Marktes neu und wirkte somit als Rule Breaker. Der Eintritt neuer Wettbewerber mit z. T. innovativen Geschäftsmodellen bewirkte im Zeitverlauf eine erneute Reformierung der Wettbewerbsregeln. Auf diese reagierte der Club jedoch nicht nachhaltig. Die Untersuchung der Stoßrichtung der Business Strategy ergab schließlich eine latente Ergänzung der bis in die 80er Jahre verfolgten Kostenschwerpunktstrategie um eine Differenzierungsstrategie. Dieser strategischen Erweiterung waren vor allem die Steigerung des wahrgenommenen Kundennutzens durch die Faktoren Exklusivität und Aktualität des Angebotsprogramms zuträglich.

Zum Nachweis von strategischer Rigidität im Bertelsmann Buchclub wurde als erstes Element die strategische Kontinuität auf den Ebenen des Geschäftsmodells, der Corporate sowie der Business Strategy gezeigt. 
Ob trotz dynamischer Marktverhältnisse keine strategischen Änderungen vorgenommen wurden und somit strategische Rigidität vorliegt, soll im nun folgenden Kapitel anhand einer detaillierten Umweltanalyse geprüft werden.

\subsubsection{Umweltdynamik}

Der Club Bertelsmann als Buchgemeinschaft ist weder eindeutig dem herstellenden, noch dem vertreibenden Buchhandel zuzuordnen und bewegt sich somit im weiteren Wettbewerbsumfeld des Buchmarktes. ${ }^{450}$ Aus diesem Grund muss eine Umweltanalyse nicht nur die unmittelbare Konkurrenz zu weiteren Buchgemeinschaften beachten, sondern zusätzlich sämtliche auf dem Buchmarkt positionierte Spieler wie den stationären Bucheinzelhandel oder Versandhandelsunternehmen einschließen. ${ }^{451}$

Das Analyseraster der Wettbewerbsuntersuchung orientiert sich im Grundsatz an den fünf Wettbewerbskräften von PORTER (1999), wird jedoch in Anlehnung an SteInMAnN/SCHREYÖGG (2005) um eine weitere Kategorie ergänzt: Laut PORTER bestimmen Konkurrenten innerhalb der Branche, neue Marktteilnehmer, Substitute sowie Anbieter und Käufer die Wettbewerbssituation auf dem Markt. Für die konkrete Analyse des Buchmarktes wurde die Kategorie der neuen Marktteilnehmer mit der Kategorie der bestehenden Konkurrenten zusammengefasst, da sich in einer Längsschnittbetrachtung die Bezeichnungen von ,neuen' und ,bestehenden' Marktteilnehmern relativieren. Zusätzlich entfiel die Kategorie der Lieferanten, da eine genauere Analyse von Autoren, Schriftstellern sowie Verlagen als Lizenzgebern als für die zu beantwortende Fragestellung nicht zielführend eingestuft wurde. Im Gegensatz dazu wurde die zusätzliche Kategorie der politischen Rahmenbedingungen in die Wettbe-

450 Vgl. Kapitel 4.2.1 dieser Arbeit.

451 Die Umweltanalyse wird in dem Bewusstsein unternommen, dass es „keine objektiven Abgrenzungskriterien [gibt], die lediglich zu erschließen wären. "Steinmann, H./Schreyögg, G. (2005), S. 190. Möglich wäre es so z. B. auch, zusätzlich den Musik- oder Freizeitmarkt zu betrachten, allerdings ließ die Dominanz des Buches im Bertelsmann Buchclub eine klare Positionierung im Buchmarkt erkennen. 
werbsanalyse integriert, weil die gesetzlich verankerte Buchpreisbindung ein Spezifikum des deutschen Buchmarktes darstellt. Für die nun folgende Analyse ergaben sich somit vier Kategorien: Wettbewerb unter den Anbietern, Substitute, Käufer sowie die politischen Rahmenbedingungen.

\subsection{Wettbewerb unter den Anbietern}

Nach Beendigung des Zweiten Weltkrieges herrschte auf dem deutschen Buchmarkt eine übersichtliche Wettbewerbssituation. Die Infrastruktur im Bucheinzelhandel war schlecht ausgebaut, der Markt wurde dominiert von kleinen und mittelgroßen Bucheinzelhandelsgeschäften in eher städtischen Wohnlagen. ${ }^{452}$ Die sich anschließende Aufbruchstimmung der frühen 50er Jahre wurde von der „Demokratisierung des Buches ${ }^{453}$ getrieben: Die traditionelle Form des Produkts ,Buch’ - von der inhaltlichen Zensur befreit - entwickelte sich weiter (Taschenbuch, Paperback, billige Reihe), hinzu kamen neuartige Vertriebsformen:

Die Gründungen von Buchgemeinschaften in den 50er Jahren sorgten für das Wiederaufleben des Buchmarkts und riefen gleichwohl einen verstärkten Wettbewerb hervor. Neben klassischem Sortimentshandel und Buchgemeinschaften konkurrierten ebenfalls der Versandbuchhandel, aber auch alternative Möglichkeiten des Buchkonsums durch Buchausleihstellen wie Werks- und öffentliche Büchereien um die Leser. ${ }^{454}$ Die Pluralität der unterschiedlichen Buchverkaufsstellen erhöhte sich in den 60er Jahren, als auch Kauf- und Warenhäuser damit begannen, Bücher in ihr Sortiment aufzunehmen. Dieser Trend setzte sich in den 70er Jahren fort. Papier- und Schreibwarenläden, Geschenk- und Sportartikelläden sowie der Fotohandel, aber auch Supermärkte, Lebensmittelgeschäfte und Kaffeeröster erweiterten ihr Angebot um Bü-

\footnotetext{
452 Vgl. Heinold, W.E. (2007), S. 27, BUA-0006/73 (Marktentwicklung, 1968), S. 15.

453 Heinold, W.E. (2007), S. 27.

454 Vgl. Börsenverein (1963), S. 10.
} 
cher. ${ }^{455}$ Neben dieser Vielzahl von Nebenmärkten beherrschten bis Anfang der 80er Jahre vor allem kleinere und mittlere Bucheinzelhändler als Vollsortimenter den Buchmarkt. Diese Buchmarktstruktur veränderte sich sukzessive, beginnend Ende der 80er Jahre.

Vermehrt drangen Billiganbieter und Buchdiscounter in den Markt ein, die mit Rest- oder Sonderauflagen die handelsüblichen Preise für Bücher erheblich unterschritten. Parallel setzte sich ein Trend im stationären Sortimentshandel fort: Während sich die Anzahl der kleinen und mittleren Buchhandlungen noch erhöhte, formierte sich eine kleine Gruppe von großen Buchhandelsunternehmen zu stark wachsenden Filialisten. ${ }^{456}$ Zentrale Charakteristika der bis zum Jahr 2007 den Buchmarkt dominierenden Buchhandelsketten waren großflächige Verkaufsräume, moderne Warenpräsentationen sowie ein breit angelegter Werbeeinsatz. ${ }^{457}$

Der durch die Konzentration weniger großer Einzelhandelsunternehmen hervorgerufene Strukturwandel ${ }^{458}$ auf dem Buchmarkt wurde zusätzlich durch einen weiteren Einfluss gestärkt. Die technologische Entwicklung des Internets ermöglichte die Etablierung eines neuen Vertriebskanals, den sich viele Unternehmen zu Nutze machten. Während eine Vielzahl der klassischen Buchhandelsunternehmen und Buchgemeinschaften den Online-Vertrieb als zusätzlichen Distributionsweg nutzen, positionierten sich auf dem deutschen Markt gleichwohl reine Internet-Versender. ${ }^{459}$ Zusammen mit den mächtigen Buchhandelsketten bestimmten diese im Jahr 2007 zu großen Teilen die Entwicklung auf dem deutschen Buchmarkt. Im Folgenden soll nun detailliert auf die Entwicklung der vier zentralen Wettbewerber als Hauptkonkurrenten des Ber-

Vgl. Börsenblatt (1977, 27.05.), S. 80.

456 „Von zwei Seiten wird der deutsche Buchmarkt derzeit in die Mangel genommen: Rezession und Strukturwandel." SZ (1982, 07.02.).

457 Vgl. Heinold, W.E. (2007), S. 27, Dörrich, S. (1991), S. 59-63.

458 Vgl. Dörrich, S. (1991), S. 68-71.

459 Zwei der bekanntesten Online-Händler sind amazon.de sowie buch.de. Vgl. Börsenverein (2006), S. 3. 
telsmann Buchclubs im Jahr 2007 eingegangen werden. Hierbei soll gezeigt werden, wie sich diese schrittweise auf dem deutschen Buchmarkt etabliert haben.

\section{Die zentralen Wettbewerber: Weltbild, Hugendubel, Thalia und Amazon}

Die Verlagsgruppe Weltbild entstand ursprünglich aus dem 1948 gegründeten Zeitschriftenverlag Winfried-Werk GmbH. Durch dessen Verschmelzung mit der Weltbild Bücherdienst GmbH im Jahr 1987 entstand die Weltbild Verlag GmbH, die ab 2001 unter dem Namen Verlagsgruppe Weltbild firmiert. ${ }^{460}$ Zunächst allein im Zeitschriftengeschäft tätig, begann bereits 1972 der Auf- und Ausbau des Buchversandgeschäfts per Katalog. Im Unterschied zu Bertelsmann ist Weltbild keine Buchgemeinschaft, sondern versteht sich als freier Versender ohne Mitgliedschaftsmodell. ${ }^{461} 1994$ wurden die ersten Weltbild-Filialen eröffnet, im Jahr 1996 der Musikclub Soundsplus übernommen. ${ }^{462}$ Ein Jahr später, 1997, erweiterte Weltbild seine Vertriebswege um die OnlinePlattform weltbild.de. 1999 fusionierte Weltbild sieben eigene Buchverlage mit fünf Buchverlagen der Verlagsgruppe Georg von Holtzbrinck zur Verlagsgruppe Droemer Knaur; im selben Jahr startete Jokers, ein Buchkatalog für Sonder- und Restauflagen, der im Jahr 2000 erstmals online und zwei Jahre später mit der ersten Filialeröffnung in den stationären Buchhandel einstieg. ${ }^{463}$ Ein weiterer Meilenstein in der WeltbildUnternehmensgeschichte ist die mit der Fusion des stationären Geschäfts mit Hugendubel gegründete DBH Buch Handel GmbH \& Co. KG aus dem Jahr 2006. Unter der neu gegründeten Firma, als „Allianz starker Marken“464 bezeichnet, sind die Filialgeschäfte von Hugendubel, Buch Habel, Weiland, Schmorl \& von Seefeld, Woblthat'sche Buchhandlung, Weltbild und Jokers zusammengefasst, firmieren jedoch nach wie vor

\footnotetext{
460 Vgl. Weltbild.com (2008b).

461 Vgl. Buchreport (2004, 08.07.), S. 7; Interview BME-06021 (2006, 21.12.), S. 3.

462 Vgl. Weltbild.com (2008b).

463 Vgl. Weltbild.com (2008b).

464 Weltbild.com (2008b).
} 
unter eigenem Namen. ${ }^{465}$ Schließlich stieg Weltbild im Jahr 2007 in den Gebrauchtbuchhandel ein und ermöglichte fortan den An- und Verkauf von gebrauchten Büchern, CDs und Filmen. ${ }^{466}$

Die Buchhandlung Hugendubel wurde im Jahr 1893 als Familienunternehmen in München gegründet. ${ }^{467}$ Nach der Eröffnung drei kleinerer Filialen erfolgte im Jahr 1979 die Einweihung der ersten Großbuchhandlung Deutschlands. Innovative Charakteristika waren die großen Verkaufsfläche von über $2.000 \mathrm{qm}$ auf mehreren Ebenen, Leseinseln und ein einfacher Buchzugang für Kunden, womit Hugendubel als „Erfinder des modernen Buchkaufhauses in Deutschland“468 galt. Das großflächige Filialgeschäft wurde konsequent ausgebaut. In den folgenden Jahren expandierte Hugendubel durch eine Vielzahl von Filialeröffnungen vornehmlich in Bayern, Berlin, den neuen Bundesländern sowie Frankfurt am Main. Der Internetauftritt startete im Jahr 2001 unter hugendubel.de. ${ }^{469}$ Hugendubel unterhält zahlreiche Beteiligungen an weiteren Buchhandlungen und kooperiert durch die Fusion zu der Holding $D B H$ Buch Handels GmbH \& Co. KG mit Weltbild. ${ }^{470}$

Ähnlich wie Hugendubel in München nahm die Unternehmensgeschichte von Thalia seinen Ursprung durch die Gründung einer kleinen Buchhandlung in Hamburg im Jahre 1919.471 Die 60er Jahre waren geprägt von weiteren Filialeröffnungen in Hamburg sowie die Übernahme von einer weiteren Fachbuchhandlung. Im Jahr 1984 eröffnete - wie bereits von Hugendubel in München praktiziert - das erste großflächige Thalia-Buchhaus auf 2.000 qm Verkaufsfläche. ${ }^{472}$ Die Expansion durch weitere Er-

Vgl. Handelsblatt (2006, 18./19./20.08.), S. 11.

Vgl. Weltbild.com (2008b).

Vgl. Hugendubel.de (2008a).

Handelsblatt (2006, 18./19./20.08.), S. 11.

Vgl. Hugendubel.de (2008b).

Vgl. Hugendubel.de (2008a).

Vgl. Thalia.de (2008).

Vgl. Thalia.de (2008). 
öffnungen von großflächigen Buchhäusern und kleineren Filialgeschäften wurde in den 90er Jahren sukzessive vorangetrieben, es folgten zahlreiche Übernahmen von weiteren Buchhandlungen. Als einschneidender Meilenstein in der Unternehmensgeschichte fusionierten die Thalia Buchhandlungen im Jahr 2001 mit der zur DouglasHolding gehörenden Buchhandelsgruppe Phönix/Montanus. Das entstandene Unternehmen Thalia Holding GmbH war fortan das größte Buchhandelsunternehmen im deutschsprachigen Raum. Der Online-Versandhändler buch.de gehört damit ebenfalls zur Unternehmensgruppe. Im Jahr 2002 wurde der Online-Versandhändler BOL durch buch.de erworben. Bis zum Jahr 2007 erfolgten zahlreiche Übernahmen durch die Thalia-Gruppe als größte deutsche Buchhandelskette. ${ }^{473}$ Ähnlich wie schon im Fall von Hugendubel hat sich Thalia als „käuferorientierte Großbuchhandlung“474 fest im Markt positioniert.

Anders als die traditionsreichen deutschen Buchverlags- bzw. -handelsunternehmen drang der führende US-amerikanische Online-Buchhändler Amazon.com erstmals im Jahr 1998 in den deutschen Markt ein. ${ }^{475}$ Die Gründung von Amazon.de $G m b H$ erfolgte durch die Übernahme des deutschen Online-Buchhändlers $A B C B \ddot{u}$ cherdienst/Telebuch, und Amazon.de ist seitdem marktführender Online-Buchhändler in Deutschland. In den folgenden zehn Jahren der noch jungen Unternehmensgeschichte erfolgte eine kontinuierliche Ausweitung des Angebotsprogramms sowie der Kundenservices. So wurden im Jahr 1999 registrierten Kunden erstmals automatisierte, persönliche Buchempfehlungen unterbreitet und die ,intelligente Suchmaschine’ eingeführt. ${ }^{476}$ Das anfänglich nur Bücher umfassende Programm wurde ab dem Jahr 2000 sukzessive um CDs, Videos, Computer- und Videospiele, Geschenke, Küchenund Haushaltsartikel, Haus- und Gartenwaren, Spielwaren und Kinderprodukte sowie

\footnotetext{
473 Vgl. Thalia.de (2008).

${ }^{474}$ Heinold, W.E. (2007), S. 75.

475 Vgl. Amazon.de (2008a).

476 Vgl. Amazon.de (2008b).
} 
zuletzt im Jahr 2007 um Drogerie und Badeartikel erweitert. ${ }^{477}$ Besonders hervorzuheben sind die im Jahr 2002 erfolgte Implementierung des ,Market Place', auf dem Bücher und Non-Book-Artikel nicht nur ge-, sondern auch verkauft werden konnten. ${ }^{478}$ Durch zahlreiche Kooperationen wurden zusätzlich Dienstleistungen wie ein DVDVerleih, eine eigene VISA-Karte für Amazon.de-Kunden oder Zeitschriftenabonnements angeboten..$^{49}$

Zusammenfassend lässt sich festhalten, dass der Bertelsmann Buchclub seit Anfang der 80er Jahre einem massiven Wettbewerb über sämtliche Distributionskanäle ausgesetzt ist. Hierzu tragen vor allem der Weltbild Verlag im Kataloggeschäft, Hugendubel und Thalia in der Ladenkette sowie Amazon.de im Online-Versand bei. ${ }^{480}$ Folgende Abbildung 13 fasst die zentralen Konkurrenten aus dem Jahr 2007 zusammen. Ergänzend muss erwähnt werden, dass die klare Trennung der einzelnen Wettbewerber nach Distributionskanälen in der Praxis nicht durchzuhalten ist, da die meisten Anbieter als Multi-Channel-Unternehmen tätig sind. Nach Amazon.de ist beispielsweise bereits Weltbild Nummer zwei im Online-Buchhandel auf dem deutschen Markt. ${ }^{481}$

477 Vgl. Amazon.de (2008c); FAS (2006, 01.10.), S. 44.

478 Vgl. Amazon.de (2008d).

479 Vgl. Amazon.de (2008e); Amazon.de (2008f); Amazon.de (2008g).

480 Zur Wettbewerbssituation auf dem deutschen Buchmarkt im Jahr 2006: „Denn Sie haben erstens mal die stärksten kontinentaleuropäischen Niederlassungsformen vom Versandbuchhandel mit Amazon.com oder Amazon.de, die 20 \%ige Zuwachsraten pro Jahr realisieren in einem stagnierenden Markt. Dann haben Sie als zweites einen sehr großen mächtigen Wettbewerber Weltbild, der von 20 Millionen Anfang der 90er Jahre auf mittlerweile einen Konzern von 1,5 Milliarden EUR hochgeschossen ist. Ein großer Teil dieser Umsätze im Buchbereich mit Katalogen nicht unähnlich dem Club, aber ohne Clubmodell. Mit Online-Angeboten und jetzt neuerdings sogar mit Filialgeschäft. Und Sie haben drittens in Deutschland eine sich konzentrierende Filialistenstruktur mit Thalia im Norden, mit Hugendubel im Süden, die im Prinzip die ganze Republik aufrollen und flächendeckend überziehen mit sehr großen Buchhandlungen. Sehr - wenn man so will - attraktiven Buchhandlungen und die natürlich auch durch ihre wachsende Größe entsprechend Einkaufsmacht einsammeln und damit auch ein sehr gutes Angebot machen können." Interview BME-06021 (2006, 21.12.), S. 3. 
Abbildung 13: Hauptwettbewerber des Bertelsmann Buchclubs im Jahr 2007

\begin{tabular}{c|c|c|c} 
& Katalog & Internet & Filiale \\
\hline Mitgliedschaft & \multicolumn{3}{|c}{ Bertelsmann Buchclub } \\
\hline Keine Mitgliedschaft & Weltbild & Amazon.de & $\begin{array}{c}\text { Hugendubel } \\
\text { Thalia }\end{array}$ \\
\hline
\end{tabular}

Quelle: Eigene Darstellung.

\subsection{Substitutionsprodukte}

Wie unter Kapitel 4.1.1 erläutert, konkurriert das Buch - vor allem die in dem Programmangebot der Buchgemeinschaften vorherrschende Buchgattung der Unterhaltungsliteratur bzw. Belletristik - vornehmlich mit alternativen Möglichkeiten der Freizeitgestaltung.

Zu Beginn des Analysezeitraums 1950 fiel dem Buch nahezu konkurrenzlos ein Großteil des Bedarfs an Bedürfnisbefriedigung nach Bildung und Unterhaltung zu. Der Empfang von TV-Kanälen war nicht weit verbreitet, der Zeitschriftenmarkt überschaubar und die Erfindung des Internets noch in weiter Ferne. Hinzu kam, dass kulturelle Veranstaltungen wie Theaterbesuche vor allem bildungsfernen Bevölkerungsschichten ein Gefühl von Deplatziertheit vermittelten, sofern sie nicht mit den gängigen gesellschaftlichen Umgangsformen und kulturellen Codes vertraut waren. ${ }^{482} \mathrm{Im}$ Gegensatz dazu erlaubten Bücher und speziell die Buchclubbücher ihren Käufern einen unmittelbaren und voraussetzungslosen Zugriff auf Unterhaltung und Bildung.

Durch technische Neuerungen und eine zunehmende Medienpluralität begann sich die Situation der Nachkriegszeit bereits Mitte der 50er Jahre zu ändern. Während zunächst die Verbreitung der Schallplatte (später CD) noch wenig Einfluss auf den 
Buchverkauf ausübte, ${ }^{483}$ kannibalisierte der wachsende Einfluss des Fernsehens die zum Lesen zur Verfügung stehende Freizeit entscheidend. In den 60er Jahren trat der TV-Konsum, begleitet von dem ebenfalls steigenden Angebot im Rundfunk, in direkte Freizeitkonkurrenz zum Buch. ${ }^{484}$ Eine 1965 durchgeführte Umfrage ergab, „dass das Lesen als Freizeitbeschäftigung durch das Fernsehen zurückgedrängt wurde. Mehr als jeder dritte erwachsene Bundesbürger füllt an Werktagen die verfügbare Freizeit zur Hälfte durch Fernsehen aus und nur etwa jeder vierte Erwachsene verbringt werktags einen Teil seiner Freizeit mit Lesen. An Wochenenden verschiebt sich das Verhältnis noch zugunsten des Fernsehens. “485 Eine zunehmende Verbreitung von TVEmpfangsgeräten sowie die Ausweitung des zu empfangenden Programmangebots verstärkten die steigende Nutzung des Fernsehens weiter. Auch wenn die zur Mediennutzung verwendete Zeit absolut stieg, so verringerte sich von 1967 bis 1973 allein der zum Bücherlesen aufgewandte Anteil von 13 auf neun Prozent. ${ }^{486}$ So verdrängte das zum Massenmedium aufgestiegene Fernsehen sukzessive die bislang vom Buch geleistete Bedürfnisbefriedigung nach Bildung und Unterhaltung. ${ }^{487}$

Laut einer Verbraucheranalyse im Jahr 2005 nahm die Freizeitbeschäftigung ,Fernsehsendungen sehen' unter den ,TOP 10’ der Freizeitbeschäftigungen in Deutschland Rang zwei ein, wohingegen ,Bücher lesen’ auf Rang sieben eingestuft wird. ${ }^{488}$ Neben der starken Konkurrenz des Fernsehens ist der seit den 60er Jahren

483 Da eine Vielzahl von Buchhändlern ebenfalls Schallplatten vertrieben, steigerte der Schallplattenkonsum den Umsatz der Buchhändler zusätzlich. Vgl. Börsenblatt (1963, 26.04.), S. 660.

484 Vgl. BI (1963/03-04), S. 4. „Das Buch hat in seiner Eigenschaft als Mittel zur Bedürfnisbefriedigung zahlreiche Konkurrenten. Viele Funktionen des Buches können von Substituten teilweise oder ganz übernommen werden. Als Beispiele seien Rundfunk, Fernseher und Zeitschrift genannt. Auch sie können informieren, bilden, unterhalten, zerstreuen, sammeln und anderes mehr." Sußmann, W. (1967), S. 11.

BUA-0006/73 (Marktentwicklung,1968), S. 20.

486 Vgl. Börsenblatt (1974, 17.05.), S. 710.

487 Vgl. Dörrich, S. (1991), S. 46; „Der Verdrängungswettbewerb anderer Medien, wie zum Beispiel das Fernsehen, bleibt nicht ohne Einfluß.“ Die Welt (1982, 01.11.).

488 Vgl. Heinold, W.E. (2007), S. 54. 
ebenfalls expandierende Zeitschriften- und Illustriertenmarkt gleichermaßen in direkten Wettbewerb mit dem Buch getreten. In der bereits zitierten Rangliste belegte die Rubrik ,Zeitschriften lesen’ im Jahr 2006 Platz acht. ${ }^{489}$

\subsection{Kunden}

Ließ die Umweltanalyse bereits einen starken Wandel bezüglich der Wettbewerbsstruktur im Buchmarkt und dem Grad der Substituierbarkeit des Produkts ,Buch’ zu, so konnten auch auf Kundenseite erhebliche Änderungen im Zeitverlauf festgestellt werden. In den ersten Jahren nach der Buchclubgründung 1950 herrschte ein klarer Verkäufermarkt vor. ${ }^{490}$ Nachdem nach Kriegsende die Grundversorgung mit Gütern des täglichen Lebens abgedeckt worden war, erwachte in der Bevölkerung ein großer Nachholbedarf an Kulturgütern. ${ }^{491}$ Zunächst noch durch geringe Kaufkraft beschränkt, erwirkten steigende Einkommen in den 50er Jahren eine stark wachsende Nachfrage nach Büchern. ${ }^{492}$ Aufgrund des in weiten Teilen der Bevölkerung vorherrschenden niedrigen Bildungsstandes beschränkte die ,Schwellenangst' den ungehinderten Bucherwerb im klassischen Buchsortiment. Bislang buchferne Bevölkerungsschichten fürchteten sich, stationäre Buchhandlungen zu betreten, um die eigene Bildungsschwäche nicht vor den Augen von Verkaufspersonal und anderen Kunden offenbaren zu müssen. ${ }^{493}$

489 Sämtliche ,Top 10' der Freizeitbeschäftigungen in Deutschland sind in der Reihenfolge ihres Rankings: Musik hören; Fernsehsendungen sehen (keine Videofilme); Tageszeitung lesen; gut essen gehen; Partys feiern/mit Freunden zusammen sein; Grillen/Picknick im Freien; Bücher lesen; Zeitschriften lesen; Rad fahren; Gartenarbeit/Pflanzen. Vgl. Heinold, W.E. (2007), S. 54.

490 Vgl. Heinold, W.E. (1990), S. 80.

491 Vgl. Börsenblatt (1959, 17.03.), S. 278.

492 Vgl. Börsenblatt (1954, 04.05.), S. 256, BUA-0006/73 (Marktentwicklung, 1968), S. 13.

493 „Durch die Konfrontierung mit einer unübersehbaren Anzahl zum großen Teil unbekannter Titel und Autoren entsteht ein Gefühl der Unsicherheit, das die eigene Unkenntnis dem sachkundigen Buchhändler gegenüber nur zögernd zur Sprache kommen lässt. In dieses Unbehagen mischt sich Misstrauen dem Buchhändler gegenüber, da man seinen Ratschlägen und seiner Sachkenntnis unbeholfen gegenübersteht und sie nicht selbst überprüfen kann." Börsenblatt (1966, 07.01.), S. 21. 
Mit Anstieg des Bildungsniveaus trat die Schwellenangst in den 60er und 70er Jahren bei weiten Teilen der Bevölkerung in den Hintergrund. Gleichermaßen flachten der anfänglich rasante Nachfrageboom und die hohe Preissensibilität der Buchkäufer ab. Grund hierfür war, dass bereits ein Großteil des Prestigebedarfs an Büchern gedeckt und die leeren Bücherregale gefüllt werden konnten. ${ }^{494}$ Von 1955 bis 1962 sank der Anteil von bücherlosen Haushalten von $37 \%$ auf $20 \% .4951967$ waren es nur noch neun Prozent. ${ }^{496}$ Bildungsanstieg, höhere Einkommen und schwindende Schwellangst bewirkten zwar eine konstante und sogar steigende Buchnachfrage, allerdings hatte sich die Bevölkerung zu kritischen und selbstbewussten Lesern entwickelt: „Die Käufer selektieren heute stärker beim Buchkauf [...].“497 Diese bis heute anhaltende Entwicklung ist durch fragmentierte Interessen und differenzierte Ansprüche geprägt. ${ }^{498}$ Der ehemalige Verkäufermarkt wurde zum Käufermarkt. ${ }^{499}$

\subsection{Politische Rahmenbedingungen}

Auch wenn die Gesetzeslage zur Preisbindung auf dem deutschen Buchmarkt bereits in Kapitel 4.1 .3 behandelt wurde, so sollen nun wesentliche Entwicklungen der gesetzlichen Vereinbarungen und deren Auswirkungen auf die Buchgemeinschaftsarbeit untersucht werden. Die Konstanz der Buchpreisbindung wurde begleitet von zwei zentralen Vereinbarungen zwischen Buchgemeinschaften, Sortimentsbuchhandlungen und Verlagen: dem Hamburger und dem Potsdamer Abkommen.

Vgl. BUA-0006/73 (Marktentwicklung, 1968), S. 17.

Vgl. Börsenblatt (1966, 07.01.), S. 20.

Vgl. Börsenblatt (1970, 12.05.), S. 1016.

Handelsblatt (1982, 07.12.); vgl. Börsenblatt (1972, 03.11.), S. 2458; Börsenblatt (1973, 20.02.), S. 205; Börsenblatt (1982, 20.08.), S. 1844.

498 „Die anhaltende Spezialisierung und Diversifikation des Lesergeschmacks ist für einen Universal-Buchclub eine schwierige Herausforderung." WIC (1990/05), S. 4.

„Kennzeichen des Käufermarktes ist neben wachsenden Ansprüchen der Kunden die ebenso wachsende Fragmentierung. Immer kleinere Marktsegmente stellen immer höhere Ansprüche, während die Massenmärkte in ihrer Bedeutung zurückgehen." Heinold, W.E. (1990), S. 80. 
Das Hamburger Abkommen aus dem Jahr 1952 regelte zwischen den Vertragspartnern ,Arbeitsgemeinschaft der schöngeistigen Verleger' und ,Arbeitsgemeinschaft der schöngeistigen Sortimenter' einerseits und den „Buchgemeinschaften’ andererseits zentrale Streitpunkte. Der zeitliche Mindestabstand zwischen dem Erscheinen von Original- und Buchclubausgabe (Vorlaufzeit) wurde auf zwei Jahre, in Ausnahmefällen ein Jahr festgelegt. Zusätzlich verpflichteten sich die Buchgemeinschaften zur $\mathrm{Zu}-$ sammenarbeit mit dem Buchhandel bei Mitgliederwerbung und -belieferung sowie zum Verzicht auf zu große Preisunterschiede. Weiter wurden Grundsätze über die feste Mitgliedschaft von Buchgemeinschaften, eine besondere Kennzeichnung von Buchgemeinschaftsausgaben sowie die Zurückhaltung bei Preisvergleichen mit den Originalausgaben vereinbart. ${ }^{500}$ Die Tatsache, dass das Hamburger Abkommen vom Bundeskartellamt im Jahr 1958 für unwirksam erklärt wurde, ermöglichte zwar ein legales Zuwiderhandeln hinsichtlich der vereinbarten Bestimmungen, dennoch trug das Abkommen wesentlich zur Ausbildung von Handelsbräuchen zwischen Buchhandel, verlag und -gemeinschaft bei. In der Praxis erfolgte eine kontinuierliche Aufweichung der Hamburger Bestimmungen, was zu einem fortdauernden Konflikt zwischen Buchhandel und -gemeinschaften führte.

Im Potsdamer Abkommen aus dem Jahr 1994 wurde schließlich erneut der Umgang mit Abstandsfristen zwischen Original- und Clubausgaben geregelt. ${ }^{501}$ Es wurde eine Einigung getroffen, Buchgemeinschaftsausgaben nach sechs Monaten, in Ausnahmefällen parallel zur Originalausgabe erscheinen zu lassen. Im Jahr 2004 einigten sich der Bertelsmann Buchclub und der Börsenverein des Deutschen Buchhandels auf eine revidierte Fassung des Potsdamer Abkommens, die dem Club einräumte, seine Lizenzausgaben schon vier Monate, im Weihnachtsgeschäft bereits drei Monate nach

\footnotetext{
500 Vgl. Kollmannsberger, M. (1995), S. 82f.

501 Vgl. WIC (2003/09).
} 
Erscheinen der Originalausgabe anbieten zu dürfen. ${ }^{502}$ Trotz der sukzessiven Verkürzung der Abstandsfristen wurden diese vom Buchclub immer wieder unterschritten, so dass sich kontinuierliche Auseinandersetzungen zwischen dem Börsenverein als Vertreter des Buchhandels und dem Buchclub beobachten ließen..$^{503}$

Es lässt sich schließen, dass der generellen Konstanz der Buchpreisbindung zahlreiche Modifikationen und Nachbesserungen bezüglich der Ausnahmeregelung für Buchgemeinschaften gegenüberstanden. Am Grundsatz des gebundenen Ladenpreises und einer generellen Vorlauffrist für Clubausgaben änderte sich jedoch nichts.

\subsection{Zwischenfazit zu Umweltdynamik}

Zusammenfassend lässt sich festhalten, dass sich das Wettbewerbsumfeld im deutschen Buchmarkt von 1950 bis 2007 stark gewandelt hat. Ausgehend von übersichtlichen Wettbewerbsverhältnissen verschärfte sich die Konkurrenzsituation seit den 80er Jahren durch mitgliedschaftsfreien Versandhandel, Warenhäuser, großflächige Buchhandlungen und zuletzt Ende der 90er Jahre durch Online-Buchhändler zunehmend. Stand das Buch als Mittel zur Unterhaltung und Bildung im Jahr 1950 noch nahezu konkurrenzlos dar, so bewirkte eine sich ausbreitende Medienvielfalt die zunehmende Substituierbarkeit des Buches. Hierzu trug vornehmlich die inflationäre Ausweitung des TV-Konsums in den 70er Jahren bei. Parallel entwickelten sich weite von niedrigem Bildungsstand und Schwellenangst gekennzeichnete Bevölkerungsteile zu mündigen und selbstbewussten Buchkäufern mit stark differenzierten Interessen. Der Verkäufermarkt wandelte sich zum Käufermarkt. Einzig die Buchpreisbindung bildete eine feste Konstante über den gesamten Analysehorizont, auch wenn sich die konkreten Absprachen zwischen Buchhandel, Verlagen und Buchgemeinschaften änderten.

502 Vgl. WIC (2004/07), S. 2; Die revidierte Fassung des Potsdamer Abkommens gilt bis zum 31.12.2004, kann mit einer Einjahres-Frist gekündigt werden, frühestens jedoch zum 31.12.2010. Vgl. WIC (2004/07), S. 4.

503 Vgl. WIC (2004/07), S. 4; Handelsblatt (2003, 08.10.), S. 2. 
Es lässt sich folgern, dass sich die Buchmarktkonditionen von 1950 bis 2007 stark gewandelt haben. Vor allem seit Anfang der 80er Jahre äußerte sich die hohe Marktdynamik in einem stark umkämpften Wettbewerb.

Abbildung 14 kontrastiert die Situation auf dem deutschen Buchmarkt über die vier Analysekategorien in den Jahren 1950 und 2007.

Abbildung 14: Entwicklung des Buchmarktes

1950

2007

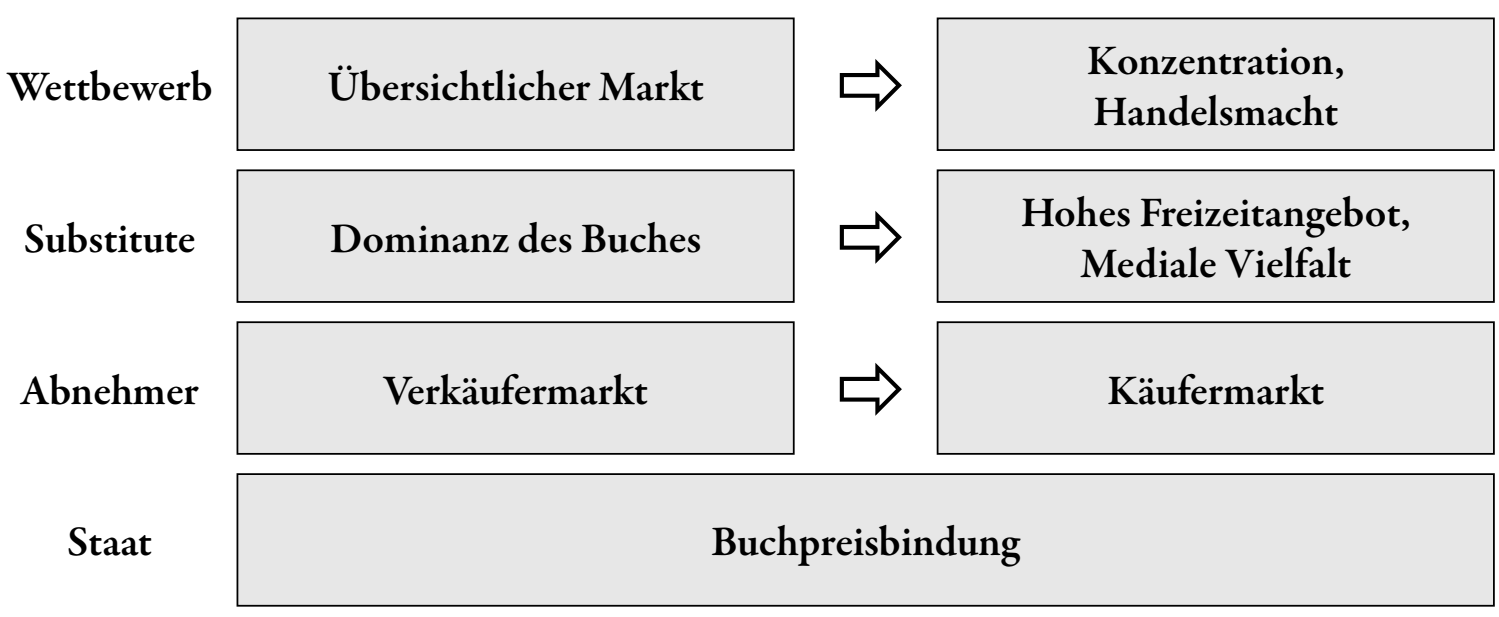

Quelle: Eigene Darstellung.

\subsubsection{Zusammenfassung}

Im vorangegangenen Kapitel 4.4 .2 sollte geprüft werden, ob im Fall des Bertelsmann Buchclubs strategische Rigidität vorliegt (Schritt 1 der Pfadanalyse). Hierzu wurde zunächst der strategische Entscheidungsverlauf des Buchclubs auf strategische Kontinuität hin untersucht. Zusammenfassend konnte auf allen drei Strategieebenen - dem Geschäftsmodell, der Corporate sowie der Business Strategy - eine im Zeitverlauf kontinuierliche Entwicklung festgestellt werden. Um zu zeigen, ob die strategische Kontinuität nicht durch stabile Umweltbedingungen zu erklären ist, wurde zusätzlich die Dynamik der wesentlichen Umweltfaktoren untersucht. Hierbei stellte sich heraus, dass sich im Laufe des Analysezeitraums weite Teile der zentralen Markt- und Wettbewerbsbedingungen auf dem deutschen Buchmarkt grundlegend gewandelt haben. 
Vor allem seit Anfang der 80er Jahre war zu beobachten, dass sich der Wettbewerb unter den Anbietern stark verschärfte, sich die Ansprüche der Kunden erhöhten und diversifizierten und sich das Produkt Buch einer verstärkten Konkurrenz mit Substitutionsprodukten ausgesetzt sah.

Bestand also noch in den 50er und 60er Jahren kein Änderungsbedarf, da die Marktverhältnisse übersichtlich waren und die Kundenansprüche bedient werden konnten, ist bereits in den 80er Jahren von einer strategischen Rigidität des Bertelsmann Buchclubs auszugehen. Obwohl sich das Wettbewerbsumfeld grundlegend änderte, setzte sich die strategische Trajektorie des Buchclubs nahezu unbeirrt fort. Aufgrund der strategischen Kontinuität trotz gleichzeitiger dynamischer Marktverhältnisse konnte somit die strategische Rigidität des Bertelsmann Buchclubs bestätigt werden.

\subsubsection{Schritt 2: Pfaddiagnose}

Mit Bestätigung des Rigiditäts-Befundes gilt es gemäß des zweischrittigen Modells der Pfadanalyse in Schritt 2, die Ausbildung der Rigidität auf die zentralen Annahmen der Pfadtheorie hin zu überprüfen. Hierzu soll untersucht werden, ob sich die festgestellte Rigidität auf Basis einer pfadabhängigen Trajektorie mit den zentralen Komponenten der Historizität, des Critical Junctures und der positiven Rückkopplung herausgebildet hat und die beobachtete Rigidität mit einem nicht bzw. nur schwer zu verlassenden Lock-In spezifiziert werden kann. Für diese Pfaddiagnose werden die einzelnen Komponenten in den nun folgenden Kapiteln anhand der strategischen Entwicklung des Bertelsmann Buchclubs intensiv diskutiert.

\subsubsection{Komponente 1: Historizität}

Die erste zu untersuchende Komponente beschreibt die historische Fundierung in der Frühphase pfadabhängiger Prozesse, die durch die beiden Elemente der Kontingenz in Form von Ergebnisoffenheit und Entscheidungspluralität - und Vorprägung, zu messen anhand von Imprints, charakterisiert wird. 
Zur Analyse der Historizität im Fall des Bertelsmann Buchclubs ist es notwendig, die Betrachtung der strategischen Entscheidungsprozesse über den eigentlichen Analysezeitraum von 1950 bis 2007 auszuweiten. Ursächlich dafür ist die Tatsache, dass das Unternehmen Bertelsmann bereits vor der Buchclubgründung im Jahr 1950 (unter dem Namen C. Bertelsmann Verlag) bestand und die frühen Verlagstätigkeiten einen nicht unwesentlichen Einfluss auf die Gründungs- und Anschlussentscheidungen ausübten. Als Reinhard Mohn die Verlagsleitung im Jahr 1947 übernahm, blickte der Verlag bereits auf eine langjährige, über mehrere Generationen gewachsene Verlagstradition zurück. Das Haus Bertelsmann war dem Buch, in seiner ursprünglichen Form eher christlich und theologisch geprägt, verpflichtet.

Entscheidenden Einfluss auf die Konstitution des Geschäftsmodells der zweistufigen Buchgemeinschaft übte die Herstellung der sog. ,Volksausgaben’ Mitte des 20. Jahrhunderts aus. Zum ersten Mal in der Verlagsgeschichte gelang es Bertelsmann, Bücher - zudem noch belletristischer Gattung - in großen Auflagen mit hohem Erfolg zu verlegen..$^{54}$ Das damalige Vorgehen, verbilligte Sonderausgaben schöngeistiger Romane in Zusammenarbeit mit dem vertreibenden Buchhandel (und erstmaliger Einschaltung von Werbemaßnahmen) zu verlegen, ähnelt schon stark der noch heute existierenden Geschäftstätigkeit des Buchclubs.

Ähnlich erfolgreich und prägend wirkte die verlegerische Tätigkeit des Bertelsmann Verlags von ,Feldpostausgaben' für die Frontsoldaten während des Zweiten Weltkriegs - auch hier erwies sich die Herstellung von Sonderausgaben in hohen Stückzahlen zu günstigen Preisen als sehr erfolgreich. ${ }^{505}$

Das Verlegen von Büchern in hohen Auflagenzahlen, Sonderausgaben, Preisvorteile - all diese Charakteristika der strategischen Konzeption des heutigen Buchclubs 
basieren somit bereits auf weit zurückliegenden Erfahrungen des C. Bertelsmann Verlags, die als Imprints auf nachfolgende Entscheidungen ausstrahlten. ${ }^{506}$

Auch wenn die Imprints bereits durch die frühe Unternehmensgeschichte des C. Bertelsmann Verlags die spätere Strategieausrichtung geprägt haben, so ist von einer deterministischen Auswirkung auf die anschließende Entscheidungstrajektorie abzusehen. Weder die Herausgabe der erfolgreichen Volksausgaben noch der Feldposttitel generierten eine derartige Schlagkraft, dass die Gründung einer zweistufigen Buchgemeinschaft die einzig zwingende Anschlussentscheidung gewesen wäre. Zum Gründungszeitpunkt wäre noch eine Vielzahl von alternativen Strategieverläufen möglich gewesen. Bertelsmann hätte sich auf seine Tradition der theologischen Literatur rückbesinnen, den Weg einer rein verlegerischen Tätigkeit einschlagen oder sich z. B. auch als vertreibender Buchhändler betätigen und Filialgeschäfte eröffnen können. Die potentielle Entscheidungsvielfalt lässt sich vor allem anhand umfangreicher Überlegungen der Gründer Fritz Wixforth und Reinhard Mohn im Vorfeld der Buchclubkonstitution belegen, deren ergebnisoffene Diskussionen ein hohes Maß an Flexibilität zeigten. ${ }^{507}$

Die Entscheidung, eine zweistufige Buchgemeinschaft ins Leben zu rufen, war somit trotz historischer Vorprägung eine Möglichkeit unter vielen, die jedoch rückblickend den strategischen Entscheidungsverlauf nachhaltig beeinflusst hat.

Zusammenfassend lässt sich schließen, dass die eigene Verlagsgeschichte die strategische Entwicklung des späteren Bertelsmann Buchclubs beeinflusste. Der strategische Entscheidungsverlauf wies jedoch noch keinen deterministischen Charakter auf, da unterschiedliche strategische Entwicklungen denkbar und möglich gewesen wären. Die strategische Entwicklung des Buchclubs war somit bereits vor dessen eigentlicher Konstitution im Jahr 1950 durch Historizität in Form von ,eingeschränkter Kontingenz' geprägt. An die Historizität der frühen Verlagsgeschichte schließt sich als zweite

\footnotetext{
506 Vgl. Stinchcombe, A.L. (1965), S. 142-193.

507 Vgl. Gööck, R. (1968), S. 1-121.
} 
zu untersuchende Komponente das Critical Juncture an, das im Folgenden analysiert werden soll.

\subsubsection{Komponente 2: Critical Juncture}

Das Critical Juncture beschreibt den Übergang von Phase I zu Phase II des DreiPhasen-Modells der Pfadentstehung und gilt somit zugleich als analytischer Startpunkt des (positiven) Rückkopplungsmechanismus. Der Logik der Pfadtheorie folgend ist ein Critical Juncture daher nicht vor dem Einsetzen der selbstverstärkenden Effekte zu bestimmen, sondern kann lediglich im Rückschluss auf Basis bereits entstandener Rückkopplungen retrospektiv determiniert werden. Die Verortung des Critical Junctures im Fall des Bertelsmann Buchclubs erfolgt an dieser Stelle somit im Vorgriff auf die im nachfolgenden Kapitel zu diskutierenden selbstverstärkenden Effekte.

Wie im Modell der Pfaddiagnose angeführt, besteht ein Critical Juncture aus einem oder mehreren kleinen Ereignissen (Small Events), deren Bedeutung sich aus dem wegweisenden Einfluss auf den anschließenden Entscheidungsverlauf ergibt. Im Fall des Bertelsmann Buchclubs zeigte sich, dass das Critical Juncture weniger auf einem Small, sondern vielmehr auf einem Big Event (welches sich allerdings aus mehreren kleinen Entscheidungen zusammensetzt) beruhte, durch das der Prozess der Pfadentstehung angestoßen wurde. Das Big Event als kritisches Ereignis stellt im Fall von Bertelsmann die Gründungsentscheidung der zweistufigen Buchclubgründung dar, auf deren Basis sich der Erfolg und die Wirkkraft der selbstverstärkenden Mechanismen nur kurze Zeit später entfalteten.

Vor allem die bislang neue Idee der Zweistufigkeit, mit dem vertreibenden Buchhandel zu kooperieren und das bestehende Netz zur eigenen Mitgliederwerbung zu nutzen, lässt sich als wegweisendes Ereignis bezeichnen, auf dessen Fundament zu nicht geringen Teilen der exponentielle, sich selbst verstärkende Anschlusserfolg fußt. Gleichermaßen zog die Entscheidung, ein nur sehr schmales Portfolio an Buchtiteln 
anzubieten, selbstverstärkende Effekte nach sich, indem auf einen immer größer werdenden Mitgliederbestand eine nahezu gleich bleibend große Menge an angebotenen Buchtiteln traf. Welche Effekte im Einzelnen durch die Gründungsentscheidungen ausgelöst wurden, soll im nun folgenden Kapitel zur positiven Rückkopplung ausführlich erörtert werden.

\subsubsection{Komponente 3: Positive Rückkopplung}

Im Rahmen der Pfaddiagnose ließen sich drei zentrale Selbstverstärkungsmechanismen ausmachen: Economies of Scale (im Zusammenspiel mit Risikominimierung), Komplementaritäts- und Lerneffekte. Da die selbstverstärkenden Mechanismen einen Kern der pfadtheoretischen Annahmen ausmachen und durch sie zu wesentlichen Teilen die strategischen Beharrungstendenzen zu erklären sind, erfolgt eine sehr detaillierte Darstellung der Analyseergebnisse. Zunächst wird die Wirkweise jedes einzelnen Mechanismus getrennt voneinander vorgestellt, um im Anschluss daran das Zusammenwirken zu verdeutlichen.

\subsection{Economies of Scale und Risikominimierung}

Im Folgenden soll ein sich selbst verstärkender Kreislauf aufgezeigt werden, auf dessen Basis die Entscheidung, das 1950 entwickelte Geschäftsmodell beizubehalten, in den Wachstumsphasen fortlaufend bestätigt wurde. Die einzelnen Säulen des Geschäftsmodells - im Einklang mit den herrschenden Marktbedingungen - führten zu steigenden Skalenerträgen, sinkendem Risiko und hohen Margen, die wiederum die Entscheidungen über die zentralen Säulen des Clubkonzepts verfestigten. Diese ,Erfolgsspirale’ begründete zu großen Teilen den wirtschaftlichen Erfolg des Clubs, bildete jedoch als Nebenprodukte Komplementaritäts- und Lerneffekte heraus, die ein Abweichen der etablierten strategischen Ausrichtung zunehmend erschwerten. In den folgenden Abschnitten wird zunächst die Wirkweise der sich selbst verstärkenden Erfolgsspirale auf Basis von Skalenerträgen (Economies of Scale) und Risikominimierung erläutert. 


\section{Strategischer Fit von Geschäftsmodell und Marktbedingungen}

Unter Rückgriff auf Kapitel 4.4.2.2 müssen zunächst die Buchmarktbedingungen zum Gründungszeitpunkt mit dem originären Lesering-Geschäftsmodell desselben Zeitpunkts zusammengeführt und abgeglichen werden. In der Nachkriegszeit herrschte auf dem deutschen Buchmarkt ein klares Unterangebot von Büchern, welches auf einen großen kulturellen Nachholbedarf in der Bevölkerung stieß. Aufgrund der zunächst noch geringen Kaufkraft und der Notwendigkeit, sich mit Gütern des täglichen Bedarfs zu versorgen, zeichneten sich die Nachfrager durch ein preissensibles Verhalten aus. Ein in weiten Teilen der Bevölkerung vorherrschender niedriger Bildungsstand begründete ein von Schwellenangst geprägtes Buchkaufverhalten. Wenig gebildete und buchferne Bevölkerungsgruppen mieden den Kontakt zu stationären Buchgeschäften und verzichteten so auf eine Möglichkeit zum Bucherwerb. Buchgemeinschaften bestanden bereits im Markt, zahlreiche Neugründungen erfolgten in den Nachkriegsjahren. Diese bedienten sich des einstufigen Vertriebsmodells und standen so in direkter Konkurrenz zum stationären Bucheinzelhandel. Als Folge unterlagen Buchgemeinschaften und Bucheinzelhandel einem angespannten und konfliktären Verhältnis.

Den beschriebenen Marktbedingungen sind das Geschäftsmodell und die strategische Positionierung des Clubs Bertelsmann zum Gründungszeitpunkt gegenüberzustellen.

Der Preissensibilität der Verbraucher wurde mit dem Aufbau des Buchlizenzgeschäfts begegnet. Buchlizenzen bereits am Markt erschienener Titel wurden von den Verlagen erworben und nach Einhaltung der vorgeschriebenen zeitlichen Abstandsfrist in veränderter Aufmachung verbilligt angeboten.

Der gesetzlich geregelten Buchpreisbindung war die Gründung einer Buchgemeinschaft mit festem Mitgliederstamm zuträglich; nur so konnte der reguläre Buchmarktpreis unterschritten werden.

Mit der Versendung eines Buchkataloges, aus dem die Mitglieder ihre Bestellungen tätigen konnten, wurde gleichermaßen der aus dem niedrigen Bildungsstand re- 
sultierenden Schwellenangst und dem noch nicht vollständig erschlossenen Buchhandelsnetz begegnet. Auch bislang buchferne Bevölkerungsteile hatten so die Chance, einen anonymen Zugang zu Büchern zu erhalten. ${ }^{508}$

Das selektive Buchangebot des Leserings trug einerseits dem Nachholbedarf an im Krieg zerstörten Buchtiteln und andererseits der Unkenntnis der bislang buchfremden Bevölkerungsgruppen Rechnung. Entsprechend wurde dem Lektorat der Buchgemeinschaft eine hohe Auswahlkompetenz zugesprochen.

Die besondere inhaltliche Sorgfalt bei der Buchauswahl wurde um eine hochwertig erscheinende Ausstattung ergänzt, so dass durch Halbledereinbände und Goldbeschriftungen auf die vielfach verbreitete Kaufmotivation des Buches als Prestigeobjekt reagiert werden konnte..$^{509}$

Eine von Bertelsmann im Jahr 1956 in Auftrag gegebene Mitgliederbefragung des Leserings bestätigte diese Ergebnisse. Die zentralen, von den Mitgliedern wahrgenommenen Clubvorteile aus dem Jahr 1956 ergaben sich - gerankt nach der Häufigkeit der Nennung - wie folgt: Die Vielseitigkeit der Auswahl (43\%), die Ausstattung der Bücher (30\%), die Vorzugspreise (18\%), die inhaltliche Auswahl der Bücher (18\%) sowie die Bestell- und Lieferkonditionen des Clubs (16\%). ${ }^{510}$ Ähnliche Ergebnisse zeigte die Frage nach den primären Beitrittsmotiven: Die Möglichkeit, Bücher

Bei der Erschließung neuer Käuferschichten wird zwischen ,Käufern mit latentem Kaufinteresse’ und ,vorwiegend am Buchbesitz interessierten Käufern' unterschieden. Vgl. BUA-0006/73 (Marktentwicklung, 1968), S. $14 f$.

509 „Durch den schnellen wirtschaftlichen Aufschwung („Wirtschaftswunder“) wurde das Buch als kulturelles Statussymbol der Arrivierten für weite Bevölkerungsschichten zum Objekt eines prestigeorientierten Wohlstandskonsums, um sich durch den zur Schau gestellten Buchbesitz soziale Geltung zu verschaffen [...]. Diesem Käuferkreis kommen die Buchclubs durch den Bezug über den Versandweg und die aufwendige Ausstattung stark entgegen.“ BUA-0006/73 (Marktentwicklung, 1968), S. 15.

510 Vgl. BUA-0041/6 (Lesering: Umfrage, 1956), S. 27. Ziel der Studie war es u. a., die Struktur der Leseringmitglieder, ihre Einstellung zum Lesering sowie die Beurteilung seiner Leistungen zu ermitteln. Vgl. BUA-0041/6 (Lesering: Umfrage, 1956), S. 2. Eine umfangreiche Beschreibung der Erhebungsmethode ist dem Dokument ebenfalls zu entnehmen. 
zu Vorzugspreisen zu beziehen (38\%), die bequeme Möglichkeit des Buchkaufs (34\%), die gute und reichhaltige Auswahl des Angebots (32\%) sowie der Wunsch, mehr zu lesen als bisher $(22 \%) .^{511}$

Schließlich gelang es durch die Einführung eines zweistufigen Vertriebssystems und damit der Integration des vertreibenden Buchhandels, das Spannungsverhältnis zwischen dem Bertelsmann Club und dem regulären Bucheinzelhandel zumindest in Teilen zu entschärfen. ${ }^{512}$

Es lässt sich folgern, dass die zentralen Säulen des Buchclub-Geschäftsmodells gleichermaßen auf die Nachfrageverhältnisse der Kundenseite nutzenstiftend wirkten und zugleich mit den bestehenden Wettbewerbsverhältnissen im Buchmarkt harmonisierten. Der ,Fit' zwischen den strategischen Säulen des Leserings und den Marktverhältnissen wird in Abbildung 15 illustriert.

Abbildung 15: Strategischer Fit zwischen Umwelt und System

\begin{tabular}{lll} 
Marktbedingungen & & Strategischer Fit des Clubs \\
\hline Preissensibilität der Kunden & $\Rightarrow$ & Verbilligte Clubausgaben \\
\hline Buch als Prestigeobjekt & $\Rightarrow$ & Hochwertige Ausstattung \\
\hline Geringer Bildungsstand & $\Rightarrow$ & Auswahlkompetenz \\
\hline Schwellenangst der Kunden & $\Rightarrow$ & Katalogversand \\
\hline Konflikt mit Bucheinzelhandel & $\Rightarrow$ & Zweistufiges Clubsystem \\
\hline
\end{tabular}

Quelle: Eigene Darstellung.

Werbe- und Vertriebskraft zur Mitgliedergenerierung

Auf der Basis dieser strategischen Ausrichtung des Buchclubs an den Markterfordernissen setzte Bertelsmann ein komplexes System von Werbe- und Vertriebsmethoden

511 Vgl. BUA-0041/6 (Lesering, Umfrage, 1956), S. 19.

512 Vgl. hierzu die Bestimmungen des Hamburger Abkommens in Kapitel 4.4.2.2.4 dieser Arbeit. 
ein, um eine hohe Anzahl von Neumitgliedern zu generieren, die Pro-Kopf-Umsätze zu steigern sowie die Verweildauer der Clubmitglieder zu verlängern. Erklärtes Ziel war die Bedienung eines Massenmarktes mit Büchern, deren Erschließung durch das Instrumentarium der aktiven Kundenwerbung sowie der Mitgliederbetreuung geleistet werden sollte: „Es genügte nicht mehr, gute Bücher auszuwählen und dann darauf zu vertrauen, dass sich das Gute von selbst durchsetzte. Nur mit Hilfe eines gut arbeitenden Vertriebsapparates und einer den modernen Gegebenheiten entsprechenden Werbung ließ sich heute noch erfolgreich verlegerische Arbeit leisten. "“513

Im Gegensatz zu der stark begrenzten Werbe- und Vertriebskraft der einstufig organisierten Buchgemeinschaften erwies sich die von Bertelsmann eingeführte Zweistufigkeit als höchst effektiv. Neben der Entspannung des konfliktären Verhältnisses zwischen Bucheinzelhandel und Buchclub konnte aus der Kooperation mit dem vertreibenden Buchhandel eine enorme Vertriebswirkung erzielt werden. ${ }^{514}$

Der neu gegründete Buchclub bediente sich des bereits etablierten Netzes des vertreibenden Buchhandels, so dass ein Großteil des Aufwands zum Aufbau eigener Werber und Belieferungsstationen umgangen werden konnte. ${ }^{515}$ Neben der Neukundengewinnung übernahm der vertreibende Buchhandel anfänglich auch die Betreuung der geworbenen Mitglieder. Diese „ungeheure Werbekraft von Tausenden von Vertriebsfirmen "516 ermöglichte innerhalb von wenigen Jahren den exponentiellen Anstieg des Mitgliedsbestands des Clubs - eine Entwicklung, die ohne die Einschaltung der fremden buchhändlerischen Vertriebsfirmen unmöglich gewesen wäre. ${ }^{517}$

Gööck, R. (1968), S. 145.

Vgl. Interview BME-07221 (2007, 18.12.), S. 10.

„Dieses zweistufige Vertriebssystem hatte den - wie sich bald zeigen sollte - unschätzbaren Vorteil, dass die werbliche Initiative bei Hunderten und später Tausenden von Buchhandlungen lag. [...] Man bediente sich eines eingespielten, erprobten Apparates.“ Gööck, R. (1968), S. 122.

16 Gööck, R. (1968), S. 145.

Vgl. Gööck, R. (1968), S. 145. 
Zusätzlich setzte Bertelsmann ein breites Spektrum an Werbeformen ein: Neben der großen Vertreterflotte, die potentielle Neukunden zu Hause besuchte, gelang im Jahr 1952 die erfolgreiche Einführung der ,Buswerbung'. Kleinere Busse wurden mit Werbeaufschriften versehen und von innen mit Bücherregalen und Sitzmöglichkeiten ausgestattet. Vertreter für den Buchclub konnten sich so flexibel an Straßen mit hohem Publikumsverkehr mobil stationieren und neue Mitglieder werben. Konflikte mit örtlichen Behörden sowie konkurrierenden Bucheinzelhandelsläden führten schließlich - nachdem die Buswerbung nahezu zwei Jahre mit großem Erfolg zur Mitgliedergewinnung beigetragen hatte - zur Einstellung derselben.

Ohne die Zweistufigkeit des Buchvertriebs und somit die bestehenden Eigentumsverhältnisse der Adressbestände zu verändern, wurde mit der Gründung der Verlagsgemeinschaft Rheda (VG Rheda) im Jahr 1954 die Verwaltungs- und Betreuungsfunktion der einzelnen Vertriebsfirmen im Haus Bertelsmann zentralisiert.

Neben der Vertreterwerbung war es so möglich, durch die dort angesiedelte ,Adressen-Zentrale' den Buchclub zentral auf schriftlichem Wege, z. B. durch Anzeigen in Zeitungen und Zeitschriften zu bewerben. Auch bei der schriftlichen, als ,Mail-Order' bezeichneten Werbeform erhielten die Vertriebsfirmen Vergütungen für Neumitglieder, sofern sie sich an den entsprechenden Werbekosten beteiligt hatten. ${ }^{518} \mathrm{Zu}$ Vertreter- und schriftlicher Werbung trat die Ladenwerbung kooperierender Bucheinzelhändler, so dass sich die vertriebliche Breitenwirkung des Buchclubs über ein weit gestreutes Spektrum von Kanälen entfalten konnte. Überdies bestand bereits seit der Gründung des Leserings die Form der Freundschaftswerbung. Mitglieder, welche erfolgreich weitere Mitglieder warben, wurden mit Prämien wie kostenlosen Büchern

518 Vgl. Beinsen-Ruf, H.-L. (1980), S. 44; Gööck, R. (1968), S. 216. In den 60er Jahren wurde auch dazu übergegangen, konzerneigene Vertreterwerbung zuzulassen. Für eine Übersicht der einzelnen Werbeformen siehe BUA-0006/464 (Ring-Werbung, 1968). 
oder Sachgeschenken belohnt. Ähnlich wie im Falle der Vertreterwerbung ließ sich mit der Freundschaftswerbung eine hohe Breitenwirkung erzielen.

Die Betreuung bestehender Mitgliedschaften erfolgte durch das Mail-Order System. Banden sich Neukunden durch die Mitgliedschaft bereits vertraglich an den Buchclub, so wurde durch zusätzliche Anreize die freiwillige Erhöhung der Verweildauer im Club zu erhöhen versucht. Die Vergabe von Treuebänden bzw. -prämien in Form von vergünstigten Clubangeboten bereits ab einjährigen Mitgliedschaften wurde als Kundenbindungsinstrument zur Verlängerung der Verweildauer eingesetzt.

Zusammenfassend lässt sich feststellen, dass das differenzierte Werbe- und Vertriebssystem von Vertreter-, schriftlicher, Laden- und Freundschaftswerbung - ergänzt um weitere Methoden der Mitgliederbetreuung - das Ziel eines stark wachsenden Mitgliederbestands erfüllte. Nur so gelang es dem Club, das exponentielle Wachstum der Anfangsjahre durch konstant steigende Mitgliederzahlen in den Folgejahren fortzusetzen.

\section{Risikominimierung durch verplante Kaufkraft}

Nachdem ausführlich erörtert wurde, wie die Harmonisierung von ClubGeschäftsmodell mit den herrschenden Marktbedingungen über ein umfangreiches Werbe- und Vertriebssystem zu stark ansteigenden Mitgliederzahlen führte, sollen im Folgenden die hieraus resultierenden Effekte der Risikominimierung dargestellt werden.

Hierzu ist es notwendig, sich zunächst die mit der Buchherstellung verbundenen Risiken im Verlagsbuchhandel vor Augen zu führen.

Aufgrund von hoher Planungsunsicherheit steht dem großen Vorfinanzierungsbedarf der Buchherstellung eine nur schlecht einschätzbare Absatzmenge gegenüber. ${ }^{519}$ Im Einklang mit der etymologischen Bedeutung des Wortes, Verlag' legt der Verlagsbuchhandel einen Großteil der Produktionskosten vor ${ }^{520}$ und trägt somit auch das

519 Vgl. Lucius, W.D.v. (1995), S. 9-11.

520 Siehe hierzu auch Fußnote 220 dieser Arbeit. 
ökonomische Risiko, sofern sich einzelne Titel schlecht verkaufen lassen bzw. als Flop erweisen. Bei einer Vielzahl von zu verlegenden Titeln ist die Nachfrage des Buchmarktes nur schwer abzuschätzen, so dass die optimale Losgrößenplanung den herstellenden Buchhandel vor häufig nur schwer zu bewältigende Herausforderungen stellt. ${ }^{521}$ Schlecht verkäufliche Titel werden entweder nach zeitlichem Abstand weit unter Ladenpreis als Restposten angeboten oder erweisen sich als gänzlich unverkäuflich. ${ }^{522}$ Die Erstellung einer verlässlichen Absatzprognose ist dabei für die Buchgattung der Belletristik z. B. im Vergleich zu wissenschaftlicher Literatur besonders anspruchsvoll, da belletristische Titel aufgrund eines signifikant größeren Absatzpotentials stark ansteigende Absatzkurven besitzen und somit die Spanne zwischen gut verkäuflichem ,Seller' und unverkäuflichem ,Flop' sehr weit ist..23

Abbildung 16: Absatzentwicklung belletristischer Bücher

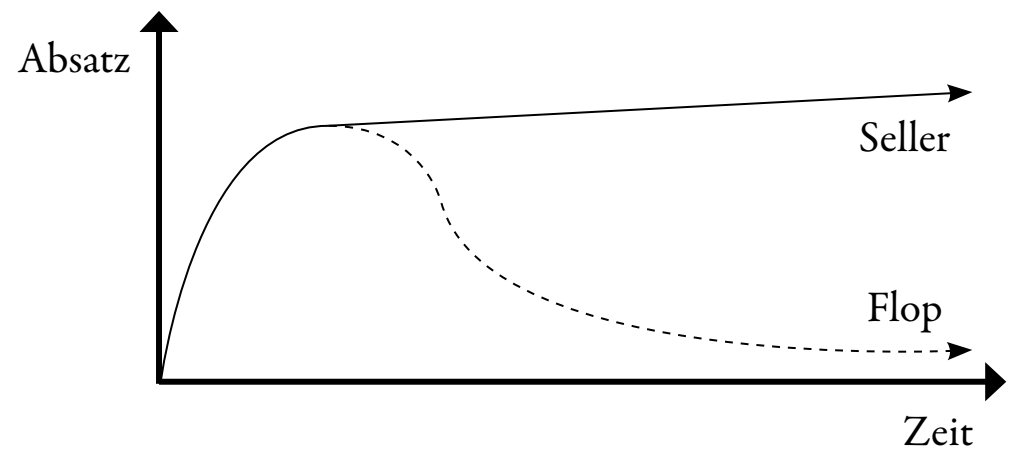

Quelle: Eigene Darstellung in Anlehnung an Schumann, M./Hess, T. (2006), S. 55.

521 „Wegen einer Vielzahl von Einflussfaktoren auf die Kaufentscheidung sind Absatzprognosen für Medienprodukte [...] relativ schwierig. Ein Beispiel für einen unerwartet hohen Absatz in der belletristischen Literatur sind die Bücher der ,Harry Potter'-Reihe." Schumann, M./Hess, T. (2006), S. 54.

522 Vgl. Hiller, H. (1966), S. $101 \mathrm{f}$.

523 Vgl. Schumann, M./Hess, T. (2006), S. 55. 
Diese hohe Planungsunsicherheit des Verlagsbuchhandels verstand der Club mit seiner Geschäftsstrategie von Buchlizenzhandel, Programmselektion und Mitgliedschaftsmodell erfolgreich zu umgehen. ${ }^{524}$

Zunächst ermöglichte der Lizenzhandel eine genaue Absatzprognose. Da der überwiegende Teil der Buchclubtitel bereits im Sortimentshandel erschienen war, ließ sich deren Erfolg auf dem ,Zweitverwerter-Markt' um ein Vielfaches besser abschätzen. ${ }^{525}$ So bestand ein Großteil des Clubprogramms aus populären Titeln, die sich im regulären Buchhandel bereits als gut verkäuflich erwiesen hatten. Nur zu einem geringen Teil wurden Imagetitel geführt, deren Absatzchancen als geringer eingestuft wurden. Bei schlecht verkäuflichen Titeln des Sortimentshandels war der Club in der Entscheidung frei, diese erst gar nicht in sein Angebotsprogramm aufzunehmen. ${ }^{526}$

Zur Reduzierung von Flop-Risiken wurden vor der endgültigen Zusammenstellung der Quartalsangebote Testkataloge an eine Auswahl von Mitgliedern als repräsentativer Querschnitt der Grundgesamtheit ausgesendet. Auf Basis der eingehenden (Test-)Bestellungen konnten Absatzpotential und Auflagenhöhe der einzelnen Titel mit hoher Verlässlichkeit prognostiziert werden. ${ }^{527}$

Als weitere Faktoren zur Verringerung des verlegerischen Risikos trugen das System der Mitgliedschaftsverpflichtung und die selektive Programmauswahl bei. ${ }^{528}$ Anders als im herkömmlichen Bucheinzelhandel war der Club in der Lage, umfangreiche Informa-tionen zu Soziodemographie, Kaufverhalten und -präferenzen seiner Mitgliecher der Buchmarkt für die Buchgemeinschaften leistet. Vgl. Hutter, M./Langenbucher, W.R. (1980), S. 14.

526 Die Auswahl der Titel durch das Lektorat der Buchgemeinschaften orientiert sich vor allem an „Bestsellerlisten (Novitäten), Berichten der Fachpresse (Börsenblatt, BuchMarkt, Buchreport) sowie der allgemeinen Presse, an ,stillen’ Erfolgen (Karl May, Kochbücher) als Steadysellern und schließlich der Bildung eines Anteils von Imagetiteln." Schönstedt, E. (1991), S. 104.

527 Vgl. Beinsen-Ruf, H.-L. (1980), S. 31f.; Hutter, M./Langenbucher, W.R. (1980), S. 14.

528 Vgl. Gööck, R. (1968), S. 112; Faulstich, W. (1979), S. 74. 
der zu generieren. Der Aufbau von Kundenkarteien und -datenbanken ermöglichte es, die Kaufakte der Mitglieder als ,gläserne Kunden’ zu antizipieren und somit die absetzbare Auflage einzelner Titel verlässlich zu prognostizieren. Aufgrund eines zunehmenden Anteils durch Freundschaftswerbung gewonnener Mitglieder ließ sich ohnehin eine verkaufsförderliche Homogenisierung der literarischen Interessen im Club feststellen. ${ }^{529}$ Die Mitglieder des Clubs waren qua Vertragsbedingungen zum quartalsmäßigen Kauf verpflichtet, so dass die Kaufkraft der Kunden im Vorfeld verplant und mit Umsatzgarantien kalkuliert werden konnte. ${ }^{530}$

Hinzu kam, dass der Club - im Vergleich zum Gesamtproduktionsvolumen auf dem deutschen Buchmarkt - eine sehr geringe Anzahl von Buchtiteln anbot. ${ }^{531}$ Wie erörtert, wurde zwar das Buchprogramm erweitert, allerdings angesichts der stark ansteigenden Titelproduktion des gesamten Buchmarkts in einer relativ geringeren Größenordnung. Es stellte sich der Effekt ein, dass sich das wachsende Gesamtkaufvolumen durch den Mitgliederanstieg auf eine zwar absolut steigende, jedoch relativ sinkende Anzahl von Buchtiteln verteilte und somit den Erfolg einzelner Titel verstärkte. ${ }^{532}$

Zusammenfassend lässt sich festhalten, dass mit steigendem Mitgliederbestand das planbare Kaufkraftvolumen bei gleichzeitiger Verringerung des verlegerischen Risikos zunahm: „Bei einer steigenden Zahl von Mitgliedern [...] kann das Lektorat verbürgen, dass für alle, auch anspruchsvolle Titel im Laufe einer vertretbaren Zeit Interessenten

Vgl. Hutter, M./Langenbucher, W.R. (1980), S. 14.

530 „Die [Bertelsmann] haben ja erst dann angefangen zu drucken, wenn sie wussten: Soundso viele Bestellungen liegen vor, und dann konnte man eben - sage ich mal - auf Anhieb erst einmal 20.000 Exemplare drucken und wusste: Höchstens auf zwei bis drei [Tausend] bleibt man sitzen, wenn überhaupt.“ Interview BE-07175 (2007, 21.09.), S. 4 sowie BG (1977/78), S. 12; Bramann, K.W. et al. (1993), S. 215; Rürup, B. et al. (1997), S. 58.

531 Siehe hierzu Kapitel 4.4.2.1.1 dieser Arbeit.

532 Aufgrund der überschaubaren Titelanzahl hat die geringe Streuung der Kaufinteressen einen wesentlichen Anteil an der Höhe des Erfolgs und des Betriebsergebnisses. Vgl. Interview BME07221 (2007, 18.12.), S. 16. 
gefunden werden. “ ${ }^{333}$ Dies erhöhte die Entscheidungsattraktivität, die bisherige - zu steigenden Mitgliederzahlen führende - strategische Ausrichtung kontinuierlich fortzusetzen. ${ }^{53}$ Es ließ sich somit ein erster positiver Rückkopplungsmechanismus erkennen.

\section{Größenvorteile durch Skalenerträge}

Neben der zirkulär-verstärkend wirkenden Risikominimierung durch die strategische Ausrichtung des Buchclubs, spielte die absolute Höhe der Verkaufsauflagen eine weitere wesentliche Rolle in der strategischen Entwicklung des Buchclubs. Um die hieraus abzuleitenden Größenvorteile des Clubs verstehen zu können, ist es hilfreich, zunächst die Kostenstruktur der Buchherstellung zu erläutern:

Bücher als Medienprodukte besitzen eine spezielle Kostenstruktur, die sich deutlich von den Kostenstrukturen anderer Industrien abhebt. ${ }^{535}$ So zeichnet sich die Buchherstellung durch einen besonders hohen Anteil an Fixkosten, d. h. von der Produktionsmenge unabhängiger Kosten, aus. ${ }^{536}$ Aufgrund dieses hohen Fixkostenanteils tritt bei der Herstellung von Büchern eine starke Degressionswirkung der (auflagen)fixen Kostenarten ein. Zu den Fixkosten der Buchproduktion zählen vor allem die Herstellungskosten für Satz, Reproduktion, Graphikerhonorare und das Einrichten der Druck- und Bindemaschinen sowie die Gemeinkosten für Gehälter, Mieten, Kreditzinsen oder Werbung. Sofern Autorenhonorare als Pauschalbeträge vereinbart worden sind, schließt der Fixkostenblock auch die Honorarkosten ein. ${ }^{537}$

Die Herstellung von nur einer Stückzahl eines gebundenen Buchs - die sog. Masterkopie oder ,First-Product-Copy' - ist die Vorlage für alle weiteren am Markt ab-

Während der durchschnittliche Fixkostenanteil des Verbrauchgüter produzierenden Gewerbes ca. $50 \%$ beträgt, nehmen die fixen Kosten bei der Buchproduktion einen durchschnittlichen Anteil von 63 \% ein. Vgl. Ludwig, J. (1997), S. 94.

537 Vgl. Blana, H. (1993), S. 30-35.

Vgl. BUA-0204/5 (Betriebsform der Buchgemeinschaft, o. D.), S. 3.

Vgl. Roszinsky-Terjung, A. (1981), S.118; Lehning, T. (2004), S. $48 f$.

Vgl. Schumann, M./Hess, T. (2006), S. 41. 
setzbaren Einheiten. ${ }^{53}$ Auf diese Masterkopie entfällt bereits ein Großteil der Gesamtkosten, wohingegen bei der Herstellung der anschließenden Kopien relativ geringfügige Kosten entstehen. ${ }^{539}$ Wird nun ein Buch in einer hohen Auflage verkauft, sinken die Stückkosten pro Exemplar, so dass sich mit steigender Auflage Stückkostendegressionseffekte einstellen.

Tabelle 12: Beispielrechnung der Stückkostendegression der Buchherstellung

\begin{tabular}{cccc}
$\begin{array}{c}\text { Auflage in } \\
\text { Exemplaren }\end{array}$ & Stückkosten in $€$ & $\begin{array}{c}\text { Auflagenfixe } \\
\text { Kosten in } €\end{array}$ & $\begin{array}{c}\text { Auflagenvariable } \\
\text { Kosten in } €\end{array}$ \\
\hline 2.000 & 6,50 & 5,00 & 1,50 \\
\hline 4.000 & 4,00 & 2,50 & 1,50 \\
\hline 6.000 & 3,16 & 1,66 & 1,50 \\
\hline 10.000 & 2,50 & 1,00 & 1,50 \\
\hline 12.000 & 2,33 & 0,83 & 1,50 \\
\hline
\end{tabular}

Quelle: Eigene Darstellung in Anlehnung an Blana, H. (1993), S. 31.

Folgende Abbildung 17 verdeutlicht diese Größenvorteile (Economies of Scale) graphisch. Es lässt sich allerdings auch erkennen, dass mit steigender Auflagenzahl die Degressionseffekte relativ abnehmen.

Vgl. Schumann, M./Hess, T. (2006), S. 41.

539 Hierzu zählen in voller Höhe die auflagenvariablen Kosten: Für die Herstellung fallen Kosten für Papier, Einbandmaterialien, Fotodruck und Bindung an, während die Gemeinkosten u. a. Auslieferungsgebühren umfassen. Vgl. Blana, H. (1993), S. 30-35. 
Abbildung 17: Stückkosten-Degressionseffekte der Buchherstellung

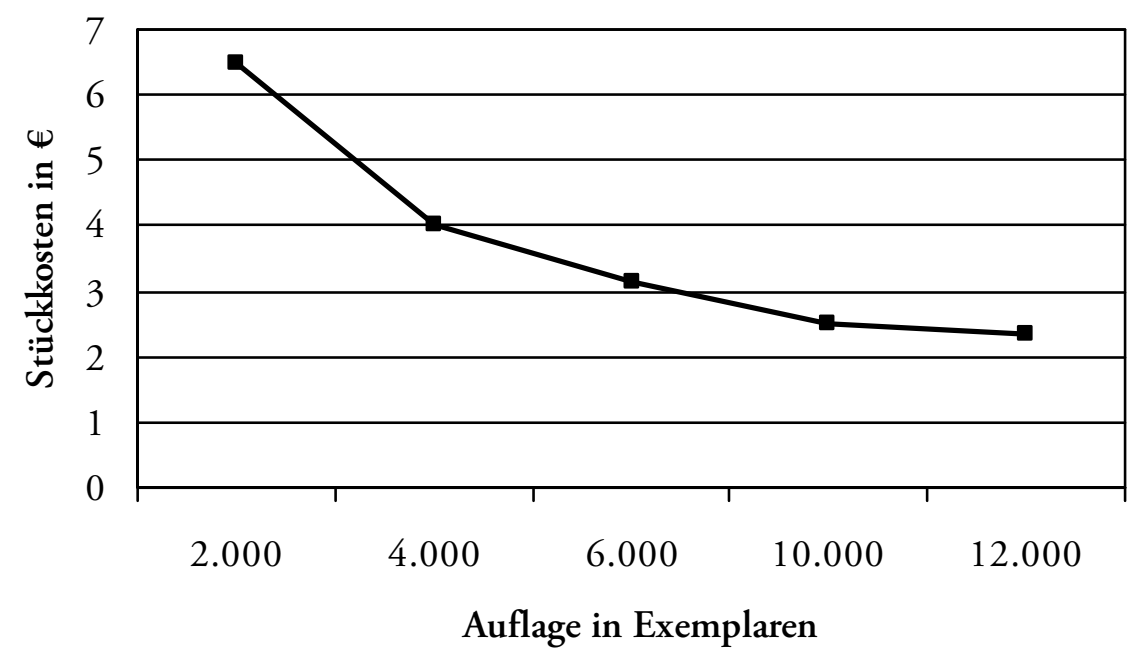

Quelle: Eigene Darstellung in Anlehnung an Blana, H. (1993), S. 32.

Die mit der Buchherstellung zu erzielenden Stückkosten-Degressionseffekte gilt es nun, auf die Geschäftsaktivitäten des Bertelsmann Clubs zu übertragen. Auch wenn die Bandbreite verkaufter Auflagen von einem Flop bis hin zu einem Bestseller sehr weit ist, lässt sich die durchschnittliche Verkaufsauflage eines belletristischen Buchtitels im Sortimentshandel auf ca. 5.000 Stück schätzen. ${ }^{540}$ Im Gegensatz dazu lag die Durchschnittsauflage im Gesamtprogramm des Clubs bei nahezu 100.000 Stück und somit um das 20fache höher. ${ }^{541}$ Die hohen Clubauflagen sind dadurch zu erklären, dass der große Mitgliederbestand des Buchclubs auf eine sehr selektive Programmauswahl traf und die Mitglieder pro Quartal zu mindestens einem Kaufakt verpflichtet waren. Vor dem Hintergrund kontinuierlich steigender Mitgliederzahlen und somit degressiver Stückkostenverläufe der Buchproduktion sind die Größenvorteile durch steigende Verkaufsauflagen immanent.

Neben dem allgemeinen Buchprogramm sind als besondere Clubleistungen die quartalsmäßig verschickten Hauptvorschlagsbände zu erwähnen:

„Mit den Hauptvorschlagsbänden stellt der Lesering jeweils die beste Leistung eines Quartals heraus. Diese Bände sind besonders geschmackvoll in Halbleder gebunden und mit rei-

540 Vgl. Schönstedt, E. (1991), S. 108.

541 Vgl. Beinsen-Ruf, H.L. (1980), S. 79; Schönstedt, E. (1993), S. 108; Faulstich, W. (1979), S. 74. 
cher Goldprägung auf dem Buchrücken versehen. Sie sind gedacht für Mitglieder, die von ihrem Recht der freien Buchwahl keinen Gebrauch machen wollen. Hat also ein Mitglied bis zum 20. des zweiten Quartalsmonat keinen Bücherwunsch geäußert, erhält es, sofern die Beiträge entrichtet wurden, automatisch die Hauptvorschlagsbände. Selbstverständlich können die HV-Bände aber auch gewählt werden." ${ }^{542}$

Diese erreichten von 1950 bis 1953 bereits Druckauflagen von bis zu 250.000 Exemplaren ${ }^{53}$ und wuchsen in den darauf folgenden Jahren stetig an. ${ }^{544}$ Die steigenden Auflagenzahlen erklärten sich durch den kontinuierlichen Mitgliederzuwachs bei einer gleichzeitig hohen Quote von verkauften Hauptvorschlagsbänden. Unter der Prämisse, dass $50 \%$ der Lesering-Mitglieder von ihrem freien Titelwahlrecht keinen Gebrauch machten und somit den Hauptvorschlagsband zugeschickt bekamen, ${ }^{545}$ ergaben sich von 1955 bis 1960 angesichts des steigenden Mitgliederbestands folgende durchschnittliche Verkaufsauflagen:

Tabelle 13: Hochrechung der Verkaufsauflage des Hauptvorschlagsbands von 1955-1960

\begin{tabular}{ccccccc}
\hline Jahr & 1955 & 1956 & 1957 & 1958 & 1959 & 1960 \\
\hline Auflage & 725.000 & 920.000 & 1.115 .000 & 1.285 .000 & 1.415 .000 & 1.450 .000 \\
\hline
\end{tabular}

Quelle: Eigene Darstellung.

Die kontinuierlich ansteigenden und im Vergleich zum regulären Buchhandel um ein mehrfaches höher liegenden Verkaufsauflagen bestätigen die Aussage, dass der Haupt-

542 BUA-0041/12 (Lesering: Anleitung für die Vertriebsfirmen, 1958), S. 3.

543 Vgl. Gööck, R. (1968), S. 135.

544 Vgl. Beinsen-Ruf, H.L. (1980), S. 79.

545 Im Jahr 1957 empfingen 50\% der Mitglieder den Hauptvorschlagsband. Die Quote sank in den darauf folgenden Jahren, so dass mit einer höheren Quote vor $1957 \mathrm{zu}$ rechen ist. Vgl. Roszinsky-Terjung, A. (1981), S. 117. Diese Berechnung bestätigt sich durch die Aussage, dass im Jahr 1954 durchschnittlich 400.000 Exemplare der Hauptvorschlagsbände gedruckt wurden. Vgl. Gööck, R. (1968), S. 150. 
vorschlagsband vor allem in der Aufbauphase des Clubs das „ökonomische Sprungbein" ${ }^{546}$ darstellte. ${ }^{547}$

Es lässt sich schließen, dass durch die strategischen Säulen der Mitgliedschaftsverpflichtung und selektiven Programmauswahl, gefördert durch die Werbe- und Vertriebskraft des zweistufigen Clubmodells, steigende Skalenerträge auf Basis eines stetig wachsenden Mitgliederbestands erwirtschaftet werden konnten. Um einen weiteren Anstieg der Skalenerträge zu generieren, erhöhte sich die Attraktivität, das bislang verfolgte Geschäftsmodell und die strategische Ausrichtung der Gesamtmarktabdeckung weiterzuverfolgen. Treibende Kraft war die Zielgröße des stetig wachsenden Mitgliederbestandes, der den Selbstverstärkungsmechanismus auf Basis steigender Skalenerträge ermöglichte.

Bestätigte sich die Buchclub-Strategie über die Rückkopplung durch sinkende Risiken und steigende Skalenerträge kontinuierlich selbst, so führte die durch das spezifische Geschäftsmodell der zweistufigen Buchgemeinschaft mögliche Kalkulation zu zusätzlichen Ertragssteigerungen. Welche Effekte die Entscheidung zur Zweistufigkeit hervorbrachte, wird im nun folgenden Kapitel beschrieben.

\section{Gewinnsteigerung durch zweistufige Kalkulation}

Zur Verdeutlichung der Vorteilhaftigkeit des von Bertelsmann gewählten zweistufigen Buchclubsystems lässt sich zunächst das Schema der Verlagskalkulation mit dem einer einstufigen Buchgemeinschaft vergleichen. ${ }^{548}$ Als ein zentraler Unterschied entfällt bei

546 Roszinsky-Terjung, A. (1981), S. 117.

547 Der 1952 aufgelegte Roman, Vom Winde verweht' von Margaret Mitchell verkaufte sich über 650.000 mal, 1956 das ,Bertelsmann Volkslexikon' 650.000 mal und 1959 Wilhelm Buschs ,Und die Moral von der Geschicht' über eine Million mal. Vgl. Gööck, R. (1968), S. 135; BUA-0006/219(2) (Volkslexikon, 1971); BI 1960/01).

548 Die einzelnen Kalkulationsrechnungen von Verlag, einstufiger und zweistufiger Buchgemeinschaft beziehen sich auf die von SCHÖNSTEDT (1997) vorgenommenen Standardkalkulationen. Der Vergleich soll die wesentlichen Unterschiede kontrastieren, so dass sich praktische Abwei- 
der einstufigen Buchgemeinschaft vom im Schnitt $35 \%$ günstigeren Verkaufspreis der Buchhandelsrabatt, da die Titel unter Umgehung des Einzelhandels direkt vom Verlag an den Leser vertrieben werden. Herstellkosten entstehen zwar prinzipiell in gleicher Höhe, sinken jedoch wie beschrieben mit steigender Auflage in Buchgemeinschaften um ein Vielfaches. Während der Verlag Autorenhonorare von durchschnittlich $10 \%$ abführen muss, werden im Kalkulationsmodell der einstufigen Buchgemeinschaft Lizenzgebühren von $4 \%$ angesetzt. Die geringeren Gebühren erklären sich aus der höheren Auflage der Buchgemeinschaftsausgaben, so dass der Verlag aus den Rechten zur Zweitverwertung noch erhebliche Gewinne, die nicht selten die des Originalverkaufs übersteigen, erwirtschaften kann. ${ }^{549}$ Schließlich müssen - im Gegensatz zur Verlagskalkulation - bei der einstufigen Buchgemeinschaft noch rund $10 \%$ des Bruttopreises für Mitgliederwerbung veranschlagt werden. In der Summe liegen bei der einstufigen Buchgemeinschaft die prozentualen Gewinne um ein Fünffaches und der absolute Gewinn pro Stück um mehr als das Dreifache höher als in der regulären Verlagskalkulation. ${ }^{50}$

Der angestellte Vergleich lässt sich nun um die von Bertelsmann verfolgte zweistufige Verlagskalkulation erweitern. Anders als im einstufigen Modell entfällt der Buchhandelsrabatt nicht, sondern wird mit $30 \%$ in die Kalkulationsrechnung aufgenommen. Grund hierfür sind die Verwaltungs- und Werbungsleistungen der als Kooperationspartner agierenden externen Vertriebsfirmen des Buchhandels. ${ }^{551}$ Vor demselben Hintergrund entfallen in der Clubkalkulation die Werbeausgaben. Die Gemeinkosten werden nur mit 25 \% veranschlagt. Für die Herstellkosten gilt dasselbe Phänomen wie schon bei der einstufigen Buchgemeinschaft: Die Masterkopie entspricht den Kosten

chungen der unternehmensspezifischen Kalkulationen vernachlässigen lassen. Vgl. Schönstedt, E. (1997), 101-109.

549 Vgl. Beinsen-Ruf, H.L. (1980), S. 82.

550 Vgl. Schönstedt, E. (1997), S. 105-107.

551 Bis zur Gründung der VG Rheda im Jahr 1954 wurde von den externen Vertriebsfirmen neben der Mitgliederwerbung für den Buchclub Bertelsmann auch die Betreuungsleistung übernommen. Vgl. hierzu ausführlich Kapitel 4.3.1.2 dieser Arbeit. 
der Verlagskalkulation, aufgrund der Fixkostendegressionseffekte liegt der prozentuale Anteil der Herstellkosten auch bei der zweistufigen Buchgemeinschaft weit unter dem der Verlagskalkulation. ${ }^{552}$ In der Summe liegt der sich aus der Clubkalkulation ergebende Gewinn abermals höher als schon bei der einstufigen Kalkulationsvariante.

Es lässt sich folgern, dass das von Bertelsmann gewählte Geschäftsmodell unter den Voraussetzungen eines steigenden Mitgliederbestandes bzw. wachsender ProKopf-Umsätze zu sich vergrößernden Gewinnspannen führte. Ursache hierfür waren das Anfallen unterschiedlicher Kostenblöcke und somit auf höheren Handelsspannen basierende Kalkulationsmöglichkeiten.

In der nachfolgenden Tabelle 14 werden die wesentlichen Unterschiede der Kalkulationsschemata von Verlag, einstufiger und zweistufiger Buchgemeinschaft in einer Beispielrechnung aufgeführt. ${ }^{553}$ Ausgangspunkt ist ein Roman mit dem Ladenpreis von $€ 38$,- der mit $35 \%$ Vergünstigung bei ein- und zweistufiger Buchgemeinschaft zu einem Preis von $€ 24,70$ angeboten wird.

Vgl. Schönstedt, E. (1997), S. 105-108.

553 Die einzelnen Werte basieren auf den Kalkulationsschemata von SCHÖNSTEDT (1997). Für die vorliegende Arbeit wurden die Währung von DM in € verändert, so dass die Höhe der absoluten Werte divergieren kann. Die relativen Werte bleiben hiervon unberührt, so dass die Beispielkalkulationen die Auswirkungen der Vertriebsform (Verlag, einstufige / zweistufige Buchgemeinschaft) auf die Gewinnhöhe in Abhängigkeit der Auflagenzahl verdeutlicht. Vgl. Schönstedt, E. (1997), S. 105-108. 


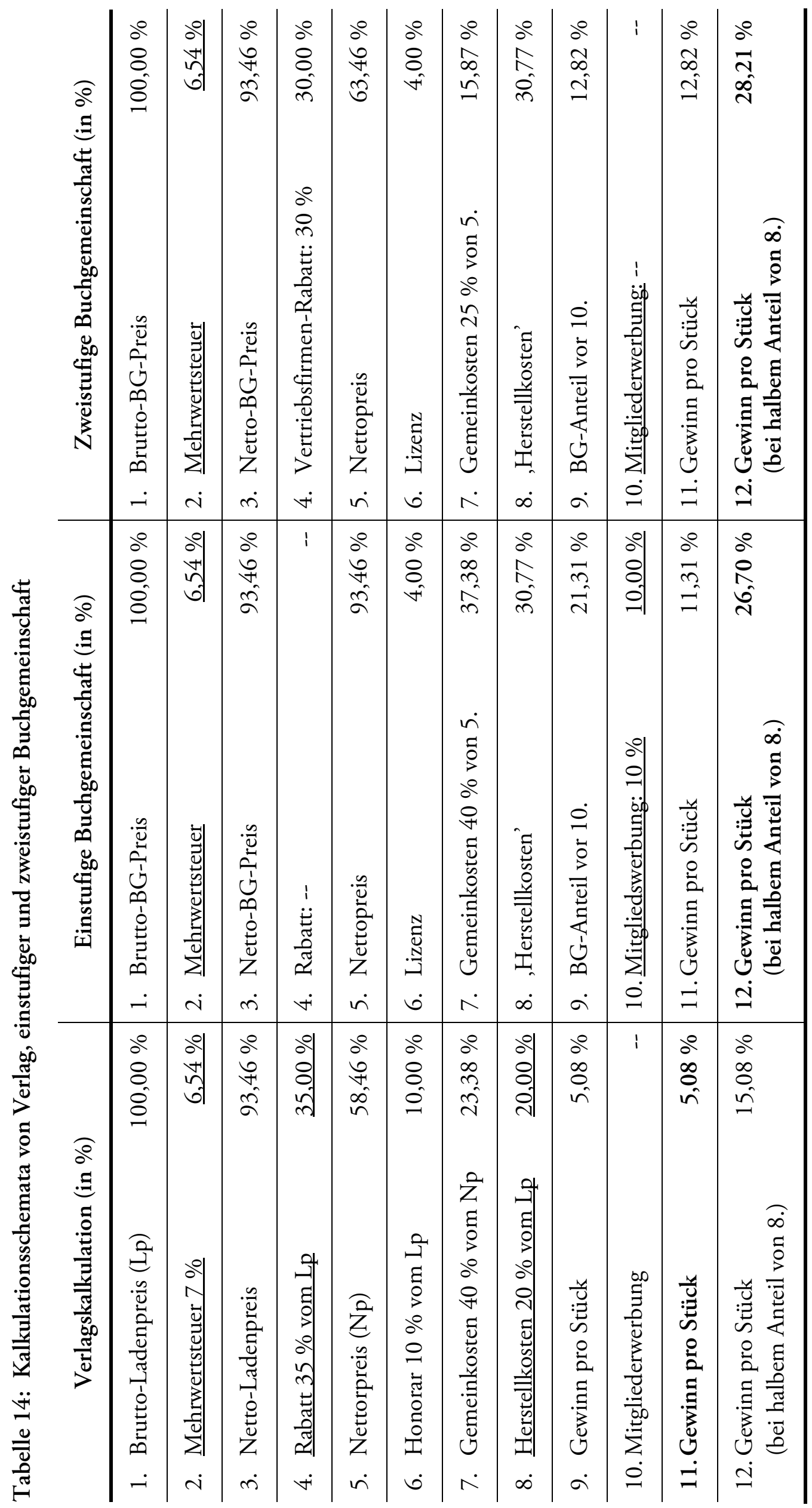




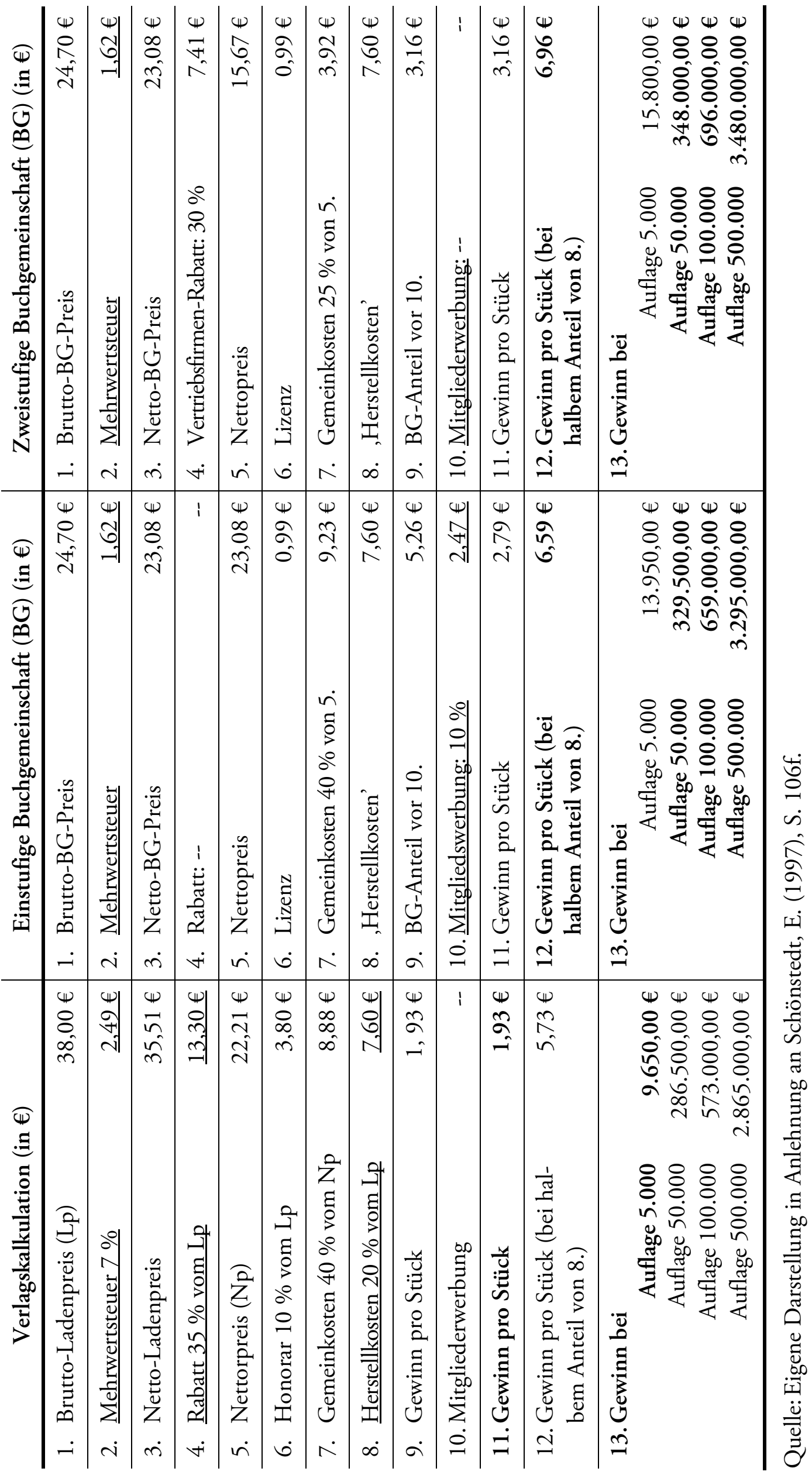


Nachfolgende Abbildung 18 stellt die Gewinnauswirkungen der verschiedenen Kalkulationsformen desselben Beispiels graphisch dar.

Es lässt sich erkennen, dass der Gewinn pro Auflage aufgrund der unterschiedlichen Kalkulationsmodi bei der zweistufigen Buchgemeinschaft (Bertelsmann Buchclub) am höchsten und beim Verlag am niedrigsten ist. ${ }^{54}$

\section{Abbildung 18: Gewinn in Abhängigkeit der Kalkulationsform und Auflage}

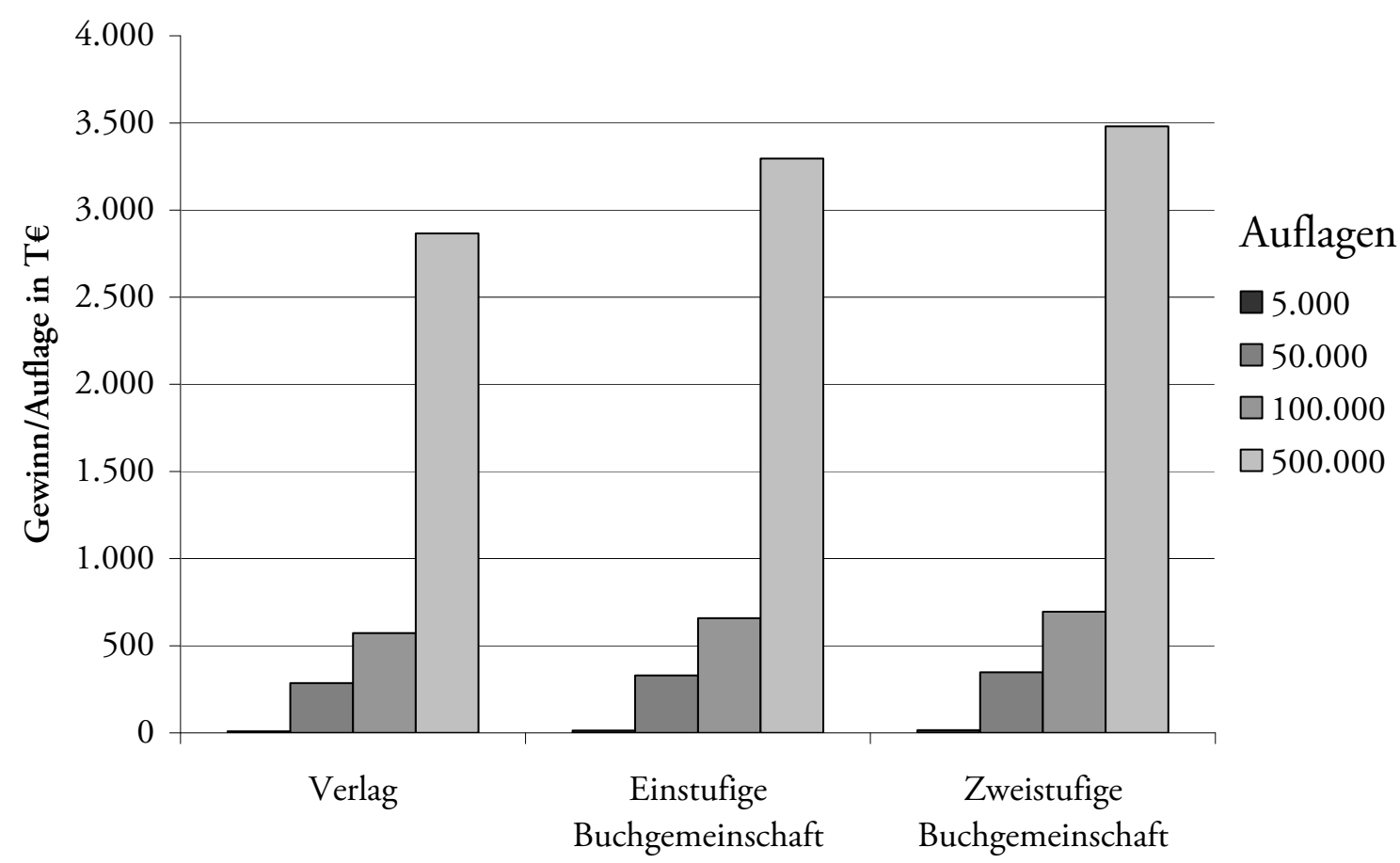

Quelle: Eigene Darstellung.

Zwei Anmerkungen sind zu ergänzen: Zum einen sind sämtliche Kalkulationen mit Auflagenzahlen von jeweils 5.000, 10.000, 100.000 und 500.000 durchgeführt wurden. Wie beschrieben, erreichen im regulären Bucheinzelhandel erscheinende Titel, die auf Basis der Verlagsrechnung kalkuliert werden, durchschnittliche Auflagen von 5.000 Kopien. Umgekehrt lag die durchschnittliche Auflage in großen Buchgemeinschaften wie dem Bertelsmann Club bei ca. 100.000. Entsprechend sind die größeren Auflagenwerte für die Verlagskalkulation sowie die kleineren Größenordnungen für die Kalkulation der Buchgemeinschaften nur aus illustrativen Gründen in die Darstellung mit aufgenommen worden. Zum zweiten basieren die Gegenüberstellungen auf standardisierten Kalkulationsschemata, die im jeweiligen Anwendungsfall divergieren können. 
Zusammenfassend lässt sich festhalten, dass sich der wirtschaftliche Erfolg des Buchclubs neben den steigenden Skalenerträgen zusätzlich durch das Geschäftsmodell der zweistufigen Buchgemeinschaft steigern ließ. Ursache dafür war eine im Gegensatz zu Verlag und einstufiger Buchgemeinschaft spezifische Kalkulationsrechnung, da der Werbe- und zunächst auch der Betreuungsaufwand für die Mitglieder von den externen Vertriebsfirmen getragen wurde. Steigende Gewinnspannen über die durch die Zweistufigkeit des Clubs ermöglichte Kalkulationsweise erhöhten somit den Anreiz, das bestehende Geschäftsmodell fortzusetzen. Die Rückkopplung auf Basis des Kalkulationsschemas verfestigte die Clubstrategie.

\subsection{Zwischenfazit zu Economies of Scale und Risikominimierung}

Im vorangegangenen Kapitel sollte gezeigt werden, durch welche selbstverstärkenden Effekte die von Bertelsmann verfolgte Strategie kontinuierlich bestätigt wurde. Es ließ sich folgender Kreislauf beschreiben:

Mit den einzelnen Säulen des Club-Geschäftsmodells (Mitgliedschaftssystem, Zweistufigkeit, Direktvertrieb, selektives Programm und Buchlizenzgeschäft) konnte ein strategischer Fit mit den herrschenden Markt- und Wettbewerbsbedingungen erzielt werden. Zusammen mit der Entscheidung, sich durch die Zweistufigkeit das bestehende Netz des verbreitenden Buchhandels zunutze zu machen, führte die strategische Positionierung des Clubs zu stark ansteigenden Mitgliederzahlen. Angesichts der spezifischen Kostenstruktur der Buchherstellung (hoher Fixkostenanteil) ließen sich über wachsende Mitgliederbestände und Auflagenhöhen steigende Skalenerträge erwirtschaften. Hinzu kam ein mit ansteigendem Mitgliederbestand vermindertes verlegerisches Risiko, da die zunehmende Kaufkraft durch das Mitgliedschaftsmodell verplant und verlässlich kalkuliert werden konnte. Aufgrund des zweistufigen Clubmodells ließ sich die steigende Ertragskraft zusätzlich durch die auf unterschiedlichen Kostenblöcken basierende Kalkulationsweise erhöhen. Hier schließt sich der Kreislauf, 
da die zur Ertragssteigerung führende strategische Ausrichtung des Clubs die Attraktivität gerade zu deren Weiterführung bestätigte und strukturell verfestigte. Steigende Gewinne wurden in Werbe- und Vertriebsbemühungen zur Erweiterung des Mitgliederbestandes und der Pro-Kopf-Umsätze reinvestiert, was zu weiter steigenden Erträgen und somit der Verfestigung des Geschäftsmodells führte. Folgende Abbildung 19 veranschaulicht die einzelnen Komponenten des sich gegenseitig verstärkenden Erfolgskreislaufs.

Abbildung 19: Erfolgsspirale des Buchclubs

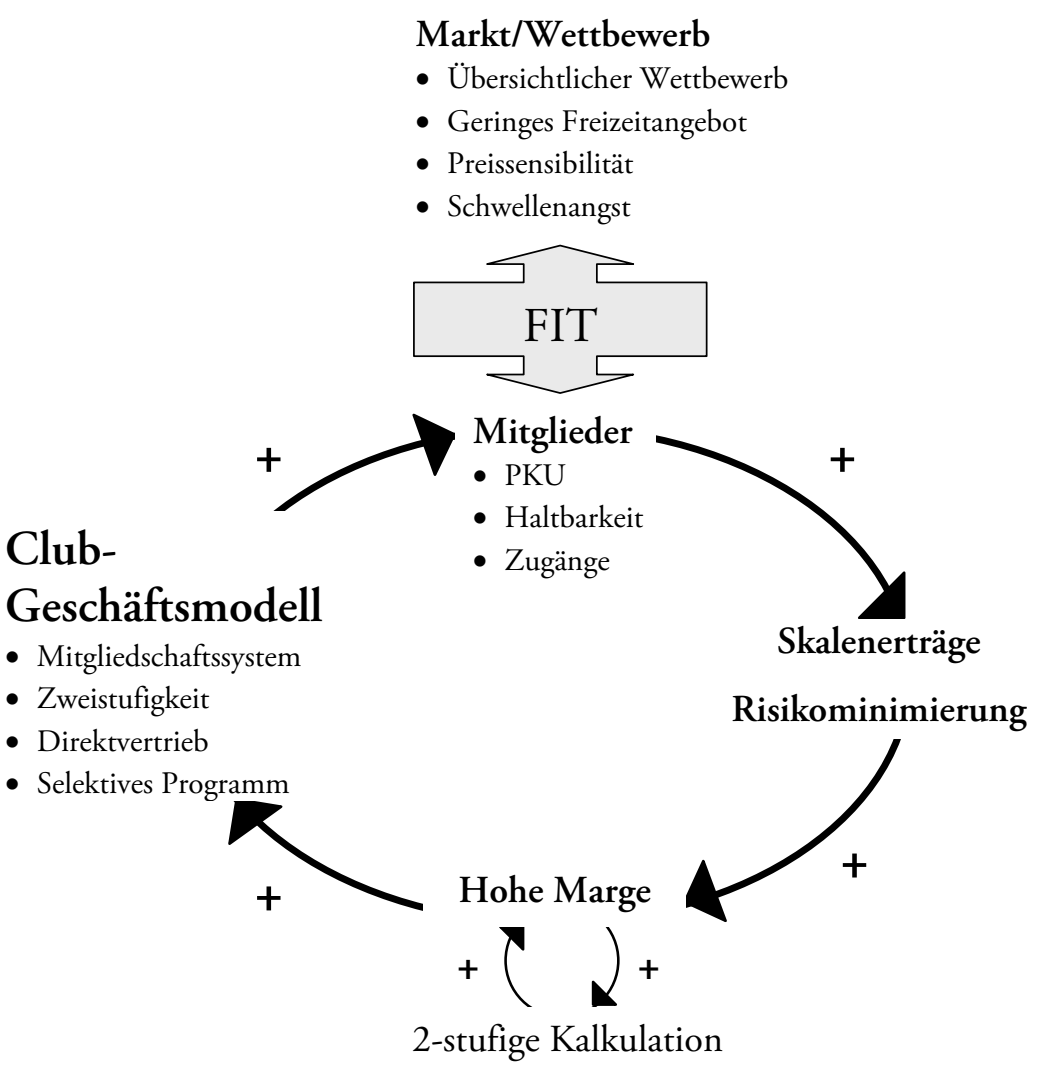

Quelle: Eigene Darstellung.

Treibende Kraft des beschriebenen Kreislaufs ist der wirtschaftliche Erfolg des Clubs. Nur durch steigende Mitgliederzahlen und Pro-Kopf-Umsätze (PKU) lassen sich über die Hebel der Fixkostendegression und Risikominderung steigende Margen generie- 
ren, die wiederum die Attraktivität, die bisherige strategische Ausrichtung beizubehalten, erhöhen.

Im Umkehrschluss und unter der Voraussetzung, dass sich die Organisation (noch) nicht im Zustand eines Lock-Ins befindet, muss allerdings auch davon ausgegangen werden, dass die selbstverstärkenden Effekte von Fixkostendegression und Risikominderung nicht mehr wirken, sofern sich Mitgliederbestand und Umsätze verringern. Unter Vorgriff auf die in Kapitel 4.4.4.4.1 zu diskutierende Mitgliederentwicklung 555 ist daher die Frage zu klären, warum sich die strategische Trajektorie des Clubs selbst bei Mitgliederverlusten und Umsatzeinbußen kontinuierlich fortsetzte. Ursächlich hierfür sind weitere Formen von selbstverstärkenden Mechanismen, deren Wirkweise in den folgenden Kapiteln erläutert wird. Das erste vorzustellende Element sind Komplementaritätseffekte.

\subsection{Komplementaritätseffekte}

Mit ansteigendem Erfolg und der Expansion des Mitgliederbestands entwickelte sich bereits in den ersten Jahren nach der Clubgründung eine komplementäre Infrastruktur um den Buchclub herum aus. Um den steigenden Bücherbedarf decken zu können, wurden vor allem Druckerei-, Lagerungs- und Auslieferungsbetriebe sowie Verlage gegründet und übernommen. Diese trugen dazu bei, den beschriebenen Erfolgskreislauf zu beschleunigen, gerieten jedoch mit dem Club in ein wechselseitiges Abhängigkeitsverhältnis. Zusätzlich bewirkte die den Cluberfolg verstärkende zweistufige Vertriebsstruktur ein zunehmendes Abhängigkeitsverhältnis zwischen Club und Vertriebsfirmen. Das komplementäre Verhältnis von konzerneigenen Druckerei- und Logistikbetrieben, Verlagen sowie externen Vertriebsfirmen bzw. Verpächtern gegenüber dem Buchclub soll in den nun folgenden Abschnitten ausführlich erörtert werden.

555 Bereits von 1962 bis 1970 setzte ein erster Mitgliederverlust ein, der sich von 1980 bis 1990 fortsetzte und in einer dritten Welle ab 1992 bis 2007 anhielt. Siehe hierzu ausführlich Kapitel 4.4.4.4.1 dieser Arbeit. 


\section{Druckerei und Logistik}

Zum Gründungszeitpunkt des Buchclubs war der Bertelsmann Verlag zunächst nicht auf die Massenherstellung von Büchern eingestellt. Die bereits dargestellte expansive Erweiterung des Mitgliederbestands führte daher sehr schnell zu Produktionsengpässen und Unterkapazitäten in der Buchherstellung.556 Das Produktionsvolumen der bestehenden Druckereibetriebe begrenzte die strategische Zielgröße des Massenvertriebs und der Gesamtmarktabdeckung. 1954 konnten nur noch 50 \% der Buchbestellungen in eigenen Druckereibetrieben hergestellt werden. ${ }^{557} \mathrm{Um}$ den steigenden Buchbedarf befriedigen zu können, wurde der technische Bereich der Mohn \& Co. erstmals im Jahr 1955 großflächig um moderne Industrieanlagen zum Buchdruck und zur Tonträgerherstellung erweitert. Mit der Ausweitung der technischen Betriebe wurde das Ziel umgesetzt, die Vergabe von Fremdaufträgen von $50 \%$ auf $30 \%$ zu reduzieren und somit einen möglichst hohen Anteil des Buchvolumens durch betriebseigene Firmen fertigen zu lassen. ${ }^{58}$ Es wurde angestrebt, eine größtmögliche Unabhängigkeit von Fremdfirmen zu erreichen. ${ }^{59}$

Der Trend setzte sich in den folgenden Jahren sukzessive fort. Der steigenden Abonnentenzahl wurde mit dem weiteren Ausbau der Produktionsanlagen begegnet, so dass im Jahr 1961 bereits 75 \% des ständig wachsenden Bücherbedarfs in den betriebseigenen Druckereien gefertigt werden konnten. ${ }^{560}$ Neben der Herstellung ent-

556 „Die stürmische Entwicklung des Leserings hatte dazu geführt, dass Setzerei, Druckerei und Buchbinderei nur noch einen Teil des Buchbedarfs selbst herstellen konnten. Dieser Anteil lag 1953 erstmals unter 50 Prozent, und auch 1954 konnte Mohn \& Co. Nur knapp die Hälfte der verlangten Bücher produzieren, etwa fünf der insgesamt notwendigen elf Millionen Bände.“ Gööck, R. (1968), S. 154; vgl. Wirtschaftswoche (1985, 14.06.).

557 Vgl. Adron, L. (1983), Vorwort.

558 Vgl. BI (1956-57/Jahreswende), S. 5.

559 Vgl. BUA-0006/218(2) (Ringgeschäft: Aktuelle Probleme, 1969), S. 8.

560 „Alle von der Leseringarbeit direkt oder indirekt befruchteten Arbeitsgebiete des Unternehmens setzten im folgenden Jahr[1961] ihren Ausbau fort.“ Gööck, R. (1968), S. 203. Im Jahr 1961 umfasste das von Mohn \& Co. hergestellte Buchvolumen fast 27 Millionen Bände pro Jahr. Vgl. BUA-0041/2 (Lesering: Entwicklung und Aufgabe, 1961), S. 14. 
standen auch logistische Einrichtungen zur Lagerung und Auslieferung der Büchermengen, so dass sich bis Ende der 60er Jahre eine stark wachsende Infrastruktur bildete, deren Ziel die Bereitstellung und Auslieferung der Buchclubbücher war. ${ }^{561}$ Aus dem 1959 gegründeten Kommissionshaus Buch und Ton ging die Vereinigte Verlagsauslieferung GmbH hervor, die schließlich aus dem Unternehmensbereich Buch- und Schallplattengemeinschaften herausgelöst und unter Bertelsmann Distribution in die separate Druck- und Industriesparte überführt wurde. ${ }^{562}$ Dienstleistungsfirmen und Buchclub entwickelten sich dabei in einem co-evolutorischen Prozess und führten zu komplementären Verflechtungen:

Durch den steigenden Buchbedarf im Buchclub stieg das Auftragsvolumen der sich stetig vergrößernden technischen und logistischen Bertelsmann Unternehmen. Diese wiederum profitierten von der durch Eigenaufträge abgedeckten konjunkturellen Unabhängigkeit und richteten sich auf die Anforderungen des Clubs aus. Die technische und logistische Unterstützung bildete somit die Basis für die Expansion des Clubs, die wiederum als ursächlich für den wirtschaftlichen Erfolg der Technik- und Dienstleistungsunternehmen von Bertelsmann zu sehen ist. ${ }^{563}$

Diese zueinander komplementäre Ausrichtung von Buchclub und Zuliefererbetrieben resultierte in einem steigenden gegenseitigen Abhängigkeitsverhältnis. So wurde im Jahr 1970 festgestellt, dass "[w]egen des z. Zt. noch hohen Abhängigkeitsgrades der Kapazitäten im Bereich der Technik und der Dienstleistungen von den Club Auf-

Vgl. Gööck, R. (1968), S. 203; Lehning, T. (2004), S. 266. Auch die Aufnahme von Schallplatten in das Programm des Leserings sowie die Gründung eines eigenen Schallplattenrings zog organisatorische Konsequenzen nach sich: Motiviert durch die Schwierigkeiten des Lizenzerwerbs und der Schallplattenproduktion wurden das Presswerk Sonopress sowie die Musikproduktionsfirma Ariola gegründet. Beide Unternehmen arbeiteten dem Lese- und Schallplattenring zu; Interessen und Arbeitsabläufe wurden aufeinander abgestimmt. Vgl. BI (1962/08-09), S. 12. Vgl. Industriemagazin (1987/06); siehe hierzu auch Kapitel 4.3.2.1 dieser Arbeit.

„Die Buchtitel werden zu etwa $70 \%$ in den Bertelsmann-eigenen Betrieben hergestellt [...] Durch diese sicheren 'Daueraufträge' ist der Konzern in der Lage, die Auslastung seiner technischen Betriebe teilweise fast unabhängig von konjunkturellen Schwankungen vorauskalkulieren zu können." Beinsen-Ruf, H.L. (1980), S. 31. 
trägen [...] größte Aufmerksamkeit und Planungsabstimmung geboten [ist]." "564 Die Tatsache, dass die Druckereibetriebe der Mohn \& Co. nur ein Drittel des gesamten Umatzvolumens aus Fremdaufträgen generierten, ${ }^{565}$ deutet exemplarisch auf das gewachsene, symbiotische Beziehungsverhältnis der Technik- und Logistikbetriebe zu den Aktivitäten des Buchclubs hin. Nicht nur der Buchclub selbst, sondern auch die um ihn gewachsene organisationale Infrastruktur, hatte sich auf den Massenvertrieb von Büchern eingerichtet: „Die Frage, ob Bertelsmann in der Bundesrepublik nicht schon zu viele Mitglieder unterhält, darf in Gütersloh nur hinter vorgehaltener Hand gestellt werden. Denn die eigenen Werbeorganisationen, Druckereien und Auslieferungsfirmen wollen beschäftigt werden. “566

Marktsättigungstendenzen und die sinkenden Mitgliederzahlen der 80er Jahre ließen schließlich den Anteil der Fremdumsätze steigen. 1985 wuchs der Anteil von Fremdaufträgen bei Mohndruck auf zwei Drittel. ${ }^{567}$ Zwar ging so der Umsatzanteil mit den Clubgeschäften von der Bertelsmann Distribution von 60 \% im Jahr 1975 zurück, lag jedoch im Jahr 1985 noch immer mit $42 \%$ auf einem sehr hohen Niveau. ${ }^{568}$

Trotz der Reduzierung von innerkonzernlichen Auftragsvergaben und somit des komplementären Abhängigkeitsverhältnisses von technischen und logistischen Betrieben stehen diese bis heute in einem engen Verbund zu den Clubgeschäften. Im Bereich der aus Druckereibetrieben und Logistik hervorgegangenen Konzerndivision Arvato, „die die komplette Logistik macht, [...] ist ein sehr hohes Volumen, ein zweistelliger Prozentsatz am Umsatz, der im Bereich Logistik an eine konzerneigene Tochter [den Buchclub] fließt. “699 Trotz der organisationalen Eigenständigkeit des Bereichs

BUA-0006/131 (Zehnjahresplanung, 1970).

Vgl. BI (1970/01), S. 10.

Vgl. Industriemagazin (1984, 15.10.).

Vgl. Adron, L. (1985), Vorwort.

Vgl. Industriemagazin (1987/06).

Interview BME-06021 (2006, 21.12.), S. 11.
} 
Arvato tragen somit die Umsätze für den Buchclub auch heute noch zum Erfolg von Arvato bei, so dass ein potentieller Wegfall der Geschäftsbeziehungen zum Club als „ein herber Schlag“" ${ }^{\text {‘70 }}$ für Arvato angesehen wird. So gilt die komplementäre Beziehung von Arvato und Buchclub („Arvato druckt und liefert fast alle Bücher “ ${ }^{(571}$ ) als ein Grund dafür, weshalb trotz dauerhafter Verluste die Geschäftsaktivitäten des Clubs in ihrer gewohnten Form fortgeführt werden. ${ }^{572}$

\section{Verlage}

Ähnlich den Druckereien und Logistikfirmen bestehen ebenfalls enge Verbindungen zwischen den Eigenverlagen und den Buchclubaktivitäten von Bertelsmann. Indem sich die „Arbeit der Buchgemeinschaften [...] naturgemäß auf die Zielsetzung und die Erfolge [der Bertelsmann-]Verlage [auswirkte]“,573 richteten sich die Verlage mit der Gründung des Buchclubs an dessen programmatischer Gestaltung aus: „Der C. Bertelsmann Verlag, Träger der Programmkompetenz, diente nur noch als Zuliefererbetrieb für den Lesering. “" 574

In den 50er und 60er Jahren basierten ca. $50 \%$ des Verlagsgruppenumsatzes auf den Aufträgen des Leserings; 575 im Buchclub selber stammten im Jahr 196040 \% des Buchangebots von Eigenverlagen. ${ }^{576}$ Auch hier lässt sich die komplementäre Beziehung zwischen eigener Verlagsgruppe und Buchclub ablesen. Die Schwierigkeiten des Lizenzerwerbs für Buchclubbücher sowie der stetig steigende Literaturbedarf erhöhten die bestehenden Eigenverlagsaktivitäten und bewirkten zahlreiche Übernahmen bzw.

Interview BME-06021 (2006, 21.12.), S. 12.

FAZ (2006, 23.08.), S.16.

572 Vgl. FAZ (2006, 23.08.), S. 16.

573 BI (1966/01-02), S. 2.

574 Langenbucher, W.R. (1985), S. 41.

575 Vgl. Böckelmann, F./Fischler, H. (2004), S. $141 f$.

576 Vgl. BI (1960/01). 
Verlagsneugründungen. ${ }^{577}$ Die Verlage konnten sich dabei auf eine Absatzgarantie des Buchclubs verlassen, so dass viele Titel der Verlagsgruppe erst durch den sicheren Vertrieb über die Buchgemeinschaft kalkulatorisch ermöglicht wurden. ${ }^{578}$ So erforderte jedes neue Buch der Verlagsgruppe ein umfangreiches Exposé, in dem die Absatzgarantie und potentielle Lizenzgewinne durch die Buchgemeinschaft enthalten waren. ${ }^{579}$

In den 60er Jahren verringerte sich der Eigenverlagsanteil im Club erscheinender Titel auf $15 \%$; der Anteil stieg von 1970 bis 1980 jedoch wieder auf $25 \% .{ }^{580} \mathrm{Im}$ folgenden Jahrzehnt stabilisierte sich der Eigenverlagsanteil weiter und verfestigte sich auf 20 bis $30 \%$ im Jahr 1989.581 Daran „wird deutlich, dass die BertelsmannBuchclubs in zunehmendem Maße zu Abnehmern der konzerneigenen Verlage werden. Das ist ein weiterer Vorteil für die Bertelsmann-Gesamtkalkulation: Zum einen kann man mit erheblichen Gewinnen durch die Club-Ausgabe rechnen [...], zum anderen hat man die Sicherheit, dass man bei eventuellen Absatzschwierigkeiten im Sortiment die gedruckte Auflage immer noch ins Club-Programm übernehmen kann, wo sie dann mit ziemlicher Sicherheit verkauft wird.“" ${ }^{822}$ In diesem Sinne stellte die Lizenzvergabe an den Club für viele Verlage eine unverzichtbare Einnahmequelle dar, die auf der anderen Seite jedoch parallel zu zunehmenden Abhängigkeitsverhältnissen führte: 583

577 Siehe hierzu ausführlich Kapitel 4.3 dieser Arbeit.

578 „Es darf bei der Betonung des reinen Vertriebscharakters [von Buchgemeinschaften] nicht vergessen werden, dass in den Verlagen Titel auf den geplanten 'Neben'-Vertrieb durch eine Buchgemeinschaft hin gemacht werden. Besonders natürlich in Verlagen, die mit einer Buchgemeinschaft unter einem Dach zusammengefasst sind. Es ist nicht zu übersehen, dass Buchgemeinschaftspolitik das Lizenzgeschäft der Verlage beeinflusst." Hutter, M./Langenbucher, W.R. (1980), S. 13.

579 Vgl. BUA-0006/91 (Verlagsarbeit, 1968), S. 5.

580 Vgl. Beinsen-Ruf, H.L. (1980), S. 83; Schönstedt, E. (1991), S. 109.

581 Vgl. Börsen-Zeitung (1989, 02.12.).

582 Beinsen-Ruf, H.L. (1980), S. 83.

583 Vgl. Hutter, M./Langenbucher, W.R. (1980), S. 9. 
Aufgrund des hohen Buchbedarfs des Clubs sowie der Schwierigkeiten des Lizenzerwerbs für Buchclubausgaben - hierzu zählte auch der von marktbeherrschenden Firmen durchgeführte Boykott am Lesering ${ }^{584}$ - errichtete Bertelsmann selbst eine komplementäre Verlagsstruktur um den Buchclub herum. Die zahlreichen zunächst in der Verlagsgruppe, später in der Produktlinie Buch, der Buch AG und schließlich unter der Division Random House geführten Eigenverlage sind dabei im Gegenzug auf die Umsätze des Buchclubs angewiesen, da der Handel mit Club-Lizenzrechten in vielen Fällen ein wichtiges ökonomisches Standbein der Verlage darstellt. In diesem Sinne richtete sich zur ökonomischen Absicherung zunehmend die Programmgestaltung der Bertelsmann Verlage an den Erfordernissen des Buchclubs aus. ${ }^{585}$

Aufgrund des Komplementärverhältnisses zwischen Buchgemeinschaften und den Verlagshäusern strahlten langfristige Mitgliederverluste im Club unmittelbar auf die angeschlossenen Verlagshäuser aus. So reduzierte die Verlagsgruppe Bertelsmann ihre Hardcover-Buchproduktion im Jahr 1981 von rund 130 Titeln pro Jahr um 35\%. Parallel wurde der Gruenwald Verlag verkauft und das Blanvalet-Programm eingestellt. Neben gestiegenem Kostendruck und konjunkturellen Schwächen galt die sinkende Nachfrage nach Lizenzrechten aufgrund stagnierender bzw. erodierender Mitgliederzahlen der Buchgemeinschaften als Ursache für die Schwierigkeiten der Verlagsgruppe. Buchclub und Verlage standen in einem gegenseitigen Abhängigkeitsverhältnis. ${ }^{586}$

Die enge Kooperationsbeziehung zwischen Bertelsmann Verlagen und Buchclub wurde zu Beginn der 90er Jahre erstmals auch organisatorisch verankert. ${ }^{587}$ Durch die Einführung des Unternehmensbereichs ,Buch Deutschland, Österreich, Schweiz’ und wenige Jahre später des Produktlinienkonzepts ,Buch’ und der ,Buch AG’ wurden

584 Vgl. Industriemagazin $(1984,15.10$.$) .$

585 Vgl. Lehning, T. (2004), S. 113. Es wird von „unternehmerischer Einheit zwischen verlegerischer und vertrieblicher Geschäftstätigkeit" gesprochen. WIC (1991/07-08), S 2.

586 Vgl. Die Zeit (1981, 22.05.); Handelsblatt (1981, 04.06.).

587 Vgl. WIC (1991/07-08), S. 2; WIC (1996/01). 
Verlage und Buchgemeinschaften auch strukturell in einer Einheit zusammengeführt, um deren Kooperation weiter zu stärken und die gemeinsamen Geschäftsaktivitäten aufeinander abzustimmen. ${ }^{58}$ Ziel war eine engere Verzahnung zwischen Buchclubs und Verlagen, die das komplementäre Abhängigkeitsverhältnis zwischen Buchgemeinschaften und Eigenverlagen verstärkte: ${ }^{589}$ „In gewisser Weise kehrt der Konzern zu seinen Anfängen zurück, als die Buchverlage in erster Linie Zulieferbetriebe für den ,Lesering’ mit entsprechendem Programm waren. “590

Auch im Fall der Verlage hat sich somit in einem co-evolutorischen Prozess ein komplementäres (innerkonzernliches) Beziehungsgeflecht herausgebildet, das die strategische Kontinuität des Buchclubs stützt: „Die Verlage brauchen den Club.“591

\section{Das zweistufige Vertriebssystem}

Unter Kapitel 4.4.2.1.1 wurden bereits ausführlich Motivation und Vorgehensweise des zweistufigen Vertriebssystems erörtert. Externe Vertriebsfirmen des Werbenden Buch-und Zeitschriftenhandels, des Reise-und Versandbuchhandels sowie der stationären Sortimentshändler warben über ihr bestehendes Vertriebsnetz Neukunden für den Buchclub. Anstatt diese Kundenadressen dem Club zu verkaufen, blieben die Vertriebsfirmen Adresseigentümer und erhielten als Ausgleich für Werbe- und Betreuungsleistungen Nutzungsentgelt bzw. umsatzabhängige Provisionszahlungen.

Diese „große, große Erfindung"“992 erweiterte die Schwungkraft zur Mitgliedergewinnung erheblich und trug wesentlich zu dem exponentiellen Wachstum des Clubs

Vgl. BG (1994/95), S. 20; SZ (1989, 05.12.); Lehning, T. (2004), S. 113.

Mit dieser Zusammenführung sollten die „regionale Kooperation von Clubs und Verlagen“, „Bündelung von Know-how" und die Schaffung von "Synergien“ gefördert werden. BG (1993/94), S. 20.

$590 \quad$ FR $(1989,28.06$.$) .$

591 WIC (2002/04); vgl. auch WIC (2006/01), S. 4.

592 Interview BME-07010 (2007, 12.03.), S. 13. 
in den 50er und 60er Jahren bei. ${ }^{593}$ Eine derartige Breitenwirkung, mit der sich der Club sukzessive von seinen Wettbewerbern abzuheben verstand, wäre ohne Zweistufigkeit nicht möglich gewesen. Mitgliederwerbungen erfordern einen hohen finanziellen Einsatz, der angesichts der angestrebten Mitgliederexpansion nicht hätte aufgebracht werden können. ${ }^{594}$

Auch im Fall der externen Vertriebsfirmen und Verpächterorganisationen ließ sich eine co-evolutorische Entwicklung mit der Clubstrategie, getrieben von sich gegenseitig verstärkenden Effekten, feststellen. Zur Mitgliedergewinnung profitierte der Club von der Werbe- und Vertriebsstärke der Verpächter, die wiederum mit wachsendem Mitgliederbestand und Pro-Kopf-Umsatz von Gewinnbeteiligungen und steigenden Provisionszahlungen des Clubs profitierten. Auf Basis des wirtschaftlichen Erfolgs baute sich so eine symbiotische, für beide Seiten attraktive Komplementärbeziehung zwischen Buchclub und externen Vertriebsfirmen auf, dessen Netz sukzessive ausgeweitet wurde.

Nachlassender Erfolg des Buchclubs in Zusammenhang mit geänderten Wettbewerbsbedingungen kehrte die aus der Komplementärbeziehung resultierende Ertragskraft jedoch in ein die Handlungsfähigkeit des Clubs einschränkendes Abhängigkeitsverhältnis um. Aufgrund der bestehenden Eigentümerstruktur der Mitgliederadressen - auch im Jahr 2007 gehörten fast die Hälfte der Clubmitglieder rechtlich nicht Bertelsmann, sondern externen Verpächtern ${ }^{595}$ - übten diese erheblichen Einfluss auf die

Vgl. Interview BME-07010 (2007, 12.03.), S. 14.

Vgl. Interview BM-08522 (2008, 05.02.), S. 4. Unterstützt wird diese Aussage durch den hohen Kostenanteil der Mitgliederwerbung von Buchgemeinschaften: „Demnach ist die Abonnentenwerbung mit ihren aufwendigen Mitteln, der Vertreter-, Anzeigen, Prospekt-, Brief- und Katalogwerbung, der Prämienvergabe für Mitgliedwerber und der Öffentlichkeitsarbeit, die alle simultan eingesetzt werden, unter den Kostenarten der Buchgemeinschaft als Buchvertriebstyp wohl an erster Stelle zu nennen. Gestützt wird diese These durch eine Auskunft des Instituts für Buchmarktforschung, Hamburg, wonach für Abonnentenwerbung bis zu zwei Dritteln des Umsatzes von einem Mitglied im ersten Jahr ausgegeben wird." Sußmann, W. (1967), S. 42. 
strategischen Entscheidungen des Clubs aus. In diesem Sinne musste „jedes Geschäft, das der Club macht, [...] immer wieder abgesprochen werden mit den Verpächtern. $\mathrm{Ob}$ es kompatibel ist, ob die Summen überführt werden können, ob die Provisionen stimmen, was auch immer. " ${ }_{596}$ Neben dem Buchprogramm müssen auch marketinggetriebene Aktivitäten wie Promotionaktionen, Reisen oder Unternehmenskooperationen nicht nur mit den Verpächtern abgesprochen, sondern auch dergestalt kalkuliert werden, dass potentielle Provisionszahlungen an die Vertriebsfirmen befriedigt werden können. ${ }^{597}$

Die Verpächter hatten somit ein erhebliches Mitspracherecht auf das Entscheidungsverhalten des Buchclubs und wirkten durch ihre Vergütungsansprüche „wie so eine Fußfessel mit einer dicken Kugel dran.“"598 Strategische Optionen und Neuerungen im Buchclub standen damit vor der Schwierigkeit, sowohl den Marktbedingungen als auch den Ansprüchen der Vertriebsfirmen gerecht zu werden - eine Situation, die sich nicht immer harmonisieren ließ: „Also [...] diese Handlungsunfähigkeit durch die Verpächter. Das hat oftmals strategische Elemente, Nebenansätze schwer behindert. " ${ }^{599}$ Auch in den Fällen von Best!Seller, Boulevard und BOL erschwerten die Vertriebsfirmen schnelle strategische Entscheidungen, um einen Konsens für ein gegenseitiges Abrechungssystem des jeweiligen Geschäftsmodells zu finden. ${ }^{600}$

Hinzu kommt, dass sich gerade der den Verpächtern gehörende Mitgliederanteil durch eine hohe Treue (durchschnittliche Verweildauer und Umsätze) dem Buchclub gegenüber auszeichnet und somit besonders rentabel ist. ${ }^{601}$ Von Bertelsmann unter-

596 Interview BM-07356 (2007, 03.09.), S. 4.

597 Vgl. Interview BM-07356 (2007, 03.09.), S. 9.

598 Interview BME-07221 (2007, 18.12.), S. 9.

599 Interview BME-07356 (2007, 03.09.), S. 4.

600 Vgl. Interview BME-07356 (2007, 03.09.), S. 4.

${ }^{601}$ Im Gegensatz dazu setzen Neumitglieder, welche z. B. durch besonders günstige Angebote (z. B. fünf Bücher für null Euro als Anreiz zum Beitritt) vom Buchclub zur kurzfristigen Bestandserhöhung geworben werden, weitaus weniger um und kündigen die Mitgliedschaft schneller. Vgl. 
nommene Anläufe, den Verpächtern ihren Adressbestand abzukaufen, konnten bislang nicht realisiert werden, da die preislichen Vorstellungen zu stark divergierten. ${ }^{602}$

Zusammenfassend lässt sich festhalten, dass die sich gegenseitig verstärkenden Effekte des zweistufigen Vertriebssystems die ökonomische Schwungkraft des Clubs vor allem in den 50er und 60er Jahren erheblich gesteigert haben. Das parallel entstandene Abhängigkeitsverhältnis lähmte den strategischen Handlungsspielraum des Clubs jedoch nachhaltig: „Man kommt aus diesem Bermuda-Dreieck nicht mehr raus.“603

\subsection{Lerneffekte}

Neben den in der Wachstumsphase des Clubs entstandenen Komplementaritäten aus Druckerei- und Logistikbetrieben, Eigenverlagen und Vertriebs- bzw. Verpächterorganisationen ließen sich ferner Lerneffekte beobachten, die gleichermaßen den strategischen Handlungsspielraum des Bertelsmann Buchclubs stark eingeschränkt haben.

Um die Auswirkungen der Lerneffekte verstehen zu können, muss zunächst erneut auf die unter Kapitel 4.4.4.3.2 beschriebene Erfolgsspirale als Ausgangspunkt verwiesen werden. Clubgeschäftsmodell und strategische Positionierung führten über steigende Auflagen und sinkendes Risiko zu Skalenerträgen und wachsenden Gewinnen, die wiederum als Rückbestätigung des eingeschlagenen strategischen Weges dienten. Analog zu dieser Entwicklung bildeten sich an das Clubgeschäft geknüpfte Lerneffekte in Form von Ergebniserwartungen aus:

Die Herstellung und der Vertrieb von Lizenzausgaben über den Buchclub sicherte Bertelsmann hohe Gewinnspannen („Das Buch ist an und für sich ein sehr schönes Produkt. Es hat eine sehr hohe Marge, im schwarz-weiss-Bereich bei den Romanen

Interview BM-07410 (2007, 02.07), S. 16f.; Interview BME-07356 (2007, 03.09.), S. 4; Interview BME-07221 (2007, 18.12.), S. 11.

602 Vgl. Interview BME-07256 (2007, 03.09.), S. 4; Interview BM-08522 (2008, 05.02.), S. 16;

Interview BMA-08593 (2008, 14.02.), S. 7.

603 Interview BME-07221 (2007, 18.12.), S. 10. 
reden wir über ca. 80 \% Marge.“" $\left.{ }^{604}\right)$. Hohe Margen und Umsatzsteigerungen zusammen mit wachsenden Marktanteilen und einer im Buchgemeinschaftsmarkt zunehmend monopolistischen Stellung erhöhten die Attraktivität, weiterhin an Clubmodell und Buchlizenzgeschäft festzuhalten. Parallel dazu verfestigte sich die Vorstellung einer hohen wirtschaftlichen Ertragskraft des Clubmodells, die als Zielvorgabe zu erfüllen war. ${ }^{605}$ Als Konsequenz dienten die „kurzfristigen Profit-Targets“" ${ }^{606}$ und „extrem rigiden Ergebniserwartungen“"607 als Referenzgröße für den Erfolg der strategischen Entwicklung des Clubs.

Das Zusammenspiel der strategischen Säulen des zweistufigen Geschäftsmodells aus Mitgliedschaftssystem, Buchlizenzgeschäft, Selektivität und Direktvertrieb diente so auf der einen Seite als Motor für den wirtschaftlichen Erfolg während der Wachstumsphase, verringerte jedoch parallel dazu die strategische Variabilität: „Dazu [strategische Neuerungen] hätten zwei Dinge nötig sein müssen: Erstens mal der Wunsch, frühzeitig in ein hochriskantes Geschäft zu investieren, von dem man nicht weiß, ob es wirklich interessant sein wird. [...] Und man hätte mutig sein müssen und hätte bereit sein müssen, das hochprofitable alte Geschäft zu kannibalisieren durch ein neues, von dem man die Profitabilität noch nicht weiß bzw. von dem man schon sicher weiß, dass es nicht so profitabel ist wie das alte. “608 In diesem Sinne hat sich der Buch-

604 Interview BME-06021 (2006, 21.12.), S. 5.

605 Der Erfolg im Clubgeschäft wird bestimmt von zwei zentralen Größen: Dem Pro-Kopf-Umsatz des einzelnen Mitglieds sowie der Höhe des Mitgliederbestandes, der sich wiederum aus den Faktoren Anzahl der Mitgliederneuwerbungen und Haltbarkeit der bestehenden Mitglieder zusammensetzt. Vgl. Interview BM-07410 (2007, 02.07.), S. 13.

606 Interview BME-06021 (2006, 21.12.), S. 8.

${ }_{607}$ Interview BME-06021 (2006, 21.12.), S. 10.

608 Interview BME-06021 (2006, 21.12.), S. 16. 
club in seiner "Geschichte an eine sehr hohe Profitabilität gewöhnt" ,, ${ }^{609}$ die es zunehmend erschwerte, alternative Unternehmensstrategien dauerhaft zu etablieren. ${ }^{610}$

Wie bereits dargelegt, erlitt die beschriebene „systemimmanente Profitabilität“611 durch die Dynamik der externen Marktbedingungen bereits in den 80er Jahren einen Bruch und führte zu sinkenden Mitgliederzahlen und Umsatzeinbußen. Als Reaktion darauf bewirkten die in der Wachstumsphase ausgebildeten kurzfristigen Ergebniserwartungen und starren Zielvorgaben lediglich kleinere Modifikationen der etablierten Strategien („Der Club hat dauernd rumgedoktert“"612) und verhinderten eine nachhaltige strategische Neuorientierung. Modifikationen der Strategie äußerten sich z. B. in der Überarbeitung der Mitgliedschaftsbedingungen, Katalogrelaunches oder der Renovierung der Clubfilialen, ohne jedoch das grundsätzliche Clubmodell zu berühren.

Zahlreiche Anläufe zur strategischen Neurorientierung (Produkt- sowie Marktentwicklung) wurden unternommen, jedoch nach kurzer Zeit wieder eingestellt. ${ }^{613}$ Beispielsweise führte die Ausweitung des Produktprogramms um Non-Book-Produkte und Dienstleistungen zwar zur Umsatzausweitung, aufgrund der relativ höheren Kosten jedoch zu sinkenden Betriebsergebnissen, so dass die Orientierung des traditionellen Buchclubs hin zu einem Medienclub nach wenigen Jahren eingestellt wurde. ${ }^{614} \mathrm{Als}$ weitere Bespiele lassen sich die Ansätze zur Markterweiterung mit Best'Seller, Boulevard und BOL anführen: „All diese Geschäfte, die man aufgezogen hat, hat man mitt-

609 Interview BME-06021 (2006, 21.12.), S. 9.

610 So wird von einer „traditionell gute[n] Rendite der Buchclubs“ gesprochen. Vgl. Die Welt (1988, 29.02.).

611 Interview BME-06021 (2006, 21.12.), S. 9.

612 Interview BE-07033 (2007, 17.07.), S. 3.

613 Vgl. Interview BME-07963 (2007, 24.04.), S. 7.

614 Vgl. Interview BM-07410 (2007, 02.07.), S. 4. Ähnlich wurde zum Thema Reisen argumentiert: „Ich war in meiner Clubzeit ein entschiedener Gegner. Das sind alles Problemzusatzgeschäfte, die sie machen, weil sie sagen: Wenn ich ein breites Spektrum habe, spreche ich vielleicht mehr Menschen an. Aber wenn Sie dann am Jahresende in die Bilanz schauen, dann stellen sie fest: Was haben wir eigentlich gemacht? Vielleicht eine schwarze Null, aber verdient wurde damit kein Geld.“ Interview BME-07010, S. 18. 
lerweile wieder eingestellt, weil es fehlte letztlich die Geduld, genügend lange in diese Geschäfte zu investieren bis sie dann auch profitabel sind, so dass man sich eigentlich auf das Kerngeschäft, das Buchclubgeschäft, konzentriert hat. “" 15 Von Best!'Seller wurde z. B. erwartet, „in einigen Jahren 100 Mill. DM Umsatz [zu] bringen“616 - ein Ziel, das nicht eingehalten werden konnte und in der Konsequenz zur Aufgabe des Geschäftsmodells führte. Die Durchsetzung kurzfristiger Ergebniserwartungen bzw. vorgaben kollidierte somit mit anfänglichen Umsatzeinbußen durch strategische Neuerungen, „auch wenn die nach einigen Jahren durchaus Potential gehabt hätten, schöne Geschäfte zu sein.“617

\subsection{Zwischenfazit zu Komplementaritäts- und Lerneffekten}

Die soeben beschriebenen Komplementaritäts- und Lerneffekte wirkten in einem zirkulär-rekursiven Feedbackprozess verstärkend auf die strategische Kontinuität des Buchclubs ein und resultierten in einer Einschränkung des strategischen Handlungsspielraums. Dieser Prozess - in Anlehnung an die in Kapitel 4.4.4.3.2 vorgestellte Erfolgsspirale als Trägheitsspirale bezeichnet - soll im Folgenden verdeutlicht werden.

Unter Bezug auf die vor allem in den 80er Jahren einsetzende und bis 2007 anhaltende Dynamik auf dem Buchmarkt geriet das Fit-Verhältnis von Club-Strategie und Marktsituation in ein zunehmendes Misfit-Verhältnis: Marktkonzentrationen und erhöhter Wettbewerb, Medienpluralität sowie steigende und differenzierte Kundenansprüche resultierten in Mitgliedererosionen des Clubs, denen zunächst durch Übernahmen von konkurrierenden Buchgemeinschaften begegnet werden konnte. Spätestens Anfang der 90er Jahre ließ sich der von sechs auf drei Millionen Mitglieder im Jahr 2007 fortschreitende Bestandsverfall nicht mehr aufhalten. ${ }^{618}$ Mit sinkenden Mit-

\footnotetext{
615 Interview BME-05021 (2006, 21.12.), S. 6.

616 Handelsblatt (1996, 25.11.), S. 15.

617 Interview BME-05021 (2006, 21.12.), S. 8.

${ }^{618}$ Siehe hierzu ausführlich Kapitel 4.4.4.4.1 dieser Arbeit.
} 
gliederzahlen wurde die Erfolgsspirale als Motor des wirtschaftlichen Wachstums außer Kraft gesetzt. Zusammen mit schwindender Kaufkraft und steigendem Absatzrisiko verringerten sich auch die Auflagenzahlen der Clubtitel, so dass die über das Clubmodell zu generierenden Skalenerträge ebenso sanken. Als Folge konnten auch über die zweistufige Kalkulationsrechnung keine steigenden Gewinne ausgewiesen werden.

Der Misfit zwischen strategischer Positionierung und Umwelt, die Mitgliedererosion, sinkende Auflagen, Skalenerträge und Umsätze hätten nach betriebswirtschaftlichem Grundverständnis zu einer Neuorientierung des Clubs führen müssen. Dennoch wies die strategische Ausrichtung des Clubs - wie in Kapitel 4.4 .2 ausführlich erörtert - auch in den Phasen wirtschaftlichen Abschwungs ein hohes Maß an Kontinuität auf. Als Ursachen hierfür konnten auf Komplementaritäts- und Lerneffekten basierende selbstverstärkende Prozesse ausgemacht werden. Während der Wachstumsphase des Club bildete sich in einem co-evolutorischen Prozess eine komplementäre Infrastruktur um den Club herum aus, deren wirtschaftliche Verflechtungen ein Abweichen der etablierten Strategie zunehmend erschwerten. Gleichermaßen bildeten sich vor dem Hintergrund der erfolgreichen Clubhistorie steigende Ergebniserwartungen an den Buchclub heraus, die sich zu rigiden Zielerwartungen verfestigten und strategische Variationen stark behinderten.

Nachfolgende Abbildung 20 fasst die Umkehrung der Erfolgsspirale und die Wirkweise von Komplementär- und Lerneffekten graphisch zusammen. 
Abbildung 20: Trägheitsspirale des Buchclubs

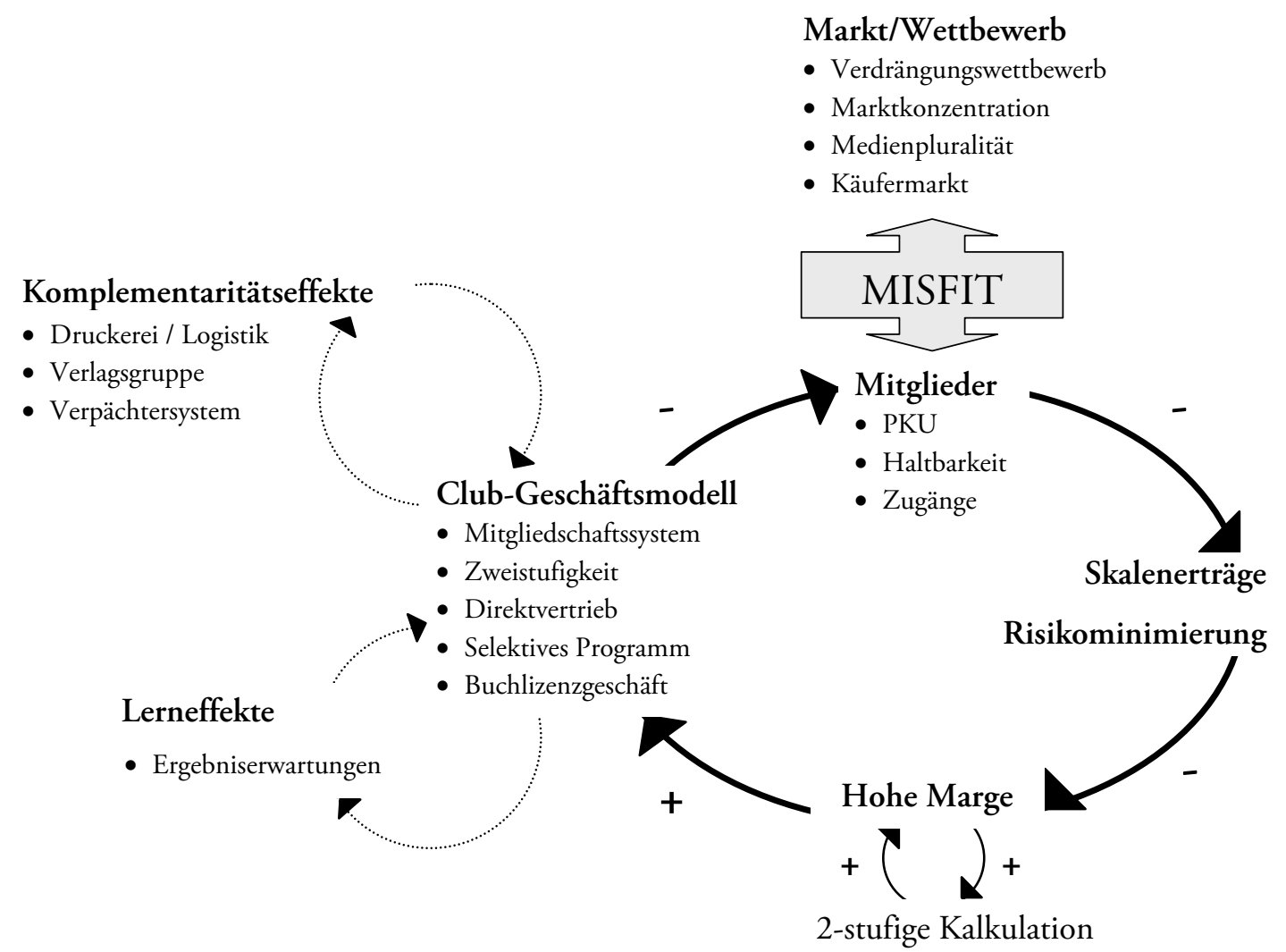

Quelle: Eigene Darstellung.

Die bisherigen Ergebnisse der Pfadanalyse lassen sich wie folgt zusammenfassen:

Im Rahmen des Rigiditätsnachweises in Kapitel 4.4.2 konnte zunächst die strategische Kontinuität des Geschäftsmodells, der Corporate und Business Strategy über den gesamten Analysezeitraum von 1950 bis 2007 gezeigt werden. Zusammen mit der dynamischen Entwicklung von Wettbewerbern, Kundenanspruch und Substitutionsprodukten auf dem deutschen Buchmarkt ließ sich feststellen, dass der kontinuierliche Strategieverlauf spätestens seit den 80er Jahren rigide ist, da nicht nachhaltig auf die Markt- und Wettbewerbsdynamik reagiert wurde.

Ursächlich für die Wandlungswiderstände war der Einfluss positiver Rückkopplung. Während in den Wachstumsphasen durch die Vergrößerung des Mitgliederbestandes steigende Skalenerträge generiert und das verlegerische Risiko minimiert werden konnten, bildeten sich parallel dazu Komplementaritäts- und Lerneffekte aus. 
Diese resultierten in einer starken Einschränkung des strategischen Handlungsspielraums im Buchclub.

Zum Abschluss der Pfaddiagnose wird im nun folgenden Kapitel die vierte und zugleich letzte Komponente - der Lock-In - untersucht. Es soll gezeigt werden, wie stark die Wirkkraft der selbstverstärkenden Mechanismen den strategischen Handlungsspielraum verengt, so dass nicht ,nur' strategische Rigidität, sondern darüber hinaus ein Lock-In vorliegt, der sich durch besonders hartnäckige Beharrungskräfte auszeichnet.

\subsubsection{Komponente 4: Lock-In}

Ein Lock-In bindet eine Organisation an ein etabliertes strategisches Muster, das sowohl von innen heraus als auch von außen nur schwer aufzubrechen ist. Die Notwendigkeit zum Aufbruch und zugleich die Inferiorität der gewählten Strategie wird durch das Element der ,Ineffizienz' beschrieben, das sich speziell im Fall von Bertelsmann an den Kennzahlen der Mitglieder- und Umsatzentwicklung messen lässt. Als zweites Element eines Lock-Ins soll mit der „Immunität' die Wandlungsresistenz der Organisation eingefangen werden, die wiederum auf das dritte Element der ,Ignoranz' im Sinne von Unkenntnis, Unbewusstsein oder Unwillen, alternative Entscheidungswege einzuschlagen, zurückzuführen ist. Schließlich beschreibt das letzte Element der ,stabilisierenden Faktoren' diejenigen Kräfte, welche - anders als die Kräfte der positiven Rückkopplung - einseitig-linear auf die strategische Trajektorie verfestigend einwirken. Alle vier Elemente sollen nun im Fall der strategischen Entwicklung des Bertelsmann Buchclubs überprüft werden.

\subsection{Ineffizienz}

Bevor auf die zentralen Erfolgsgrößen der Mitgliederentwicklung, der Umsätze sowie der Betriebsergebnisse eingegangen wird, ist voranzustellen, dass strategische Pfadabhängigkeit auch ohne ökonomische Schwierigkeiten als ineffizient zu beurteilen ist. 
Dies darf nicht mit einer potentiellen Effizienz von strategischer Kontinuität verwechselt werden, die sich analog zu Rigidität und Pfadabhängigkeit ebenfalls durch einen gleichförmigen Entscheidungsverlauf auszeichnet. Der entscheidende Unterschied liegt in der Tatsache, dass kontinuierliche, nicht pfadabhängige Entscheidungsprozesse verlassen werden können, sofern es die Umstände erfordern. Sie sind potentiell flexibel und eben nicht, locked-in'. Anders im Fall von pfadabhängigen Prozessen: Eine pfadabhängige Organisation ist aufgrund der spezifischen Prozessdynamik abhängig von ihren eigenen historischen Entscheidungsprozessen, so dass keine oder nur sehr eingeschränkte (strategische) Wahlmöglichkeiten resultieren. In diesem Sinne sind auch zunächst rein ökonomisch effiziente Pfade langfristig als problematisch zu beurteilen. Diese Problematik verschärft sich um so mehr, sobald zu der generellen Ineffizienz aufgrund strategischer Abhängigkeitsverhältnisse ökonomische Ineffizienz hinzukommt. Letztere soll am Fall von Bertelsmann analysiert werden.

Die ökonomische Entwicklung des Bertelsmann Buchclubs - gemessen an der Mitgliederentwicklung - lässt sich grob in die drei Phasen von 1950 bis 1970, von 1970 bis 1990 sowie von 1990 bis 2007 unterteilen. Alle drei Phasen verlaufen nach einem ähnlichen Muster. An das anfängliche, meist steile Wachstum schließt sich eine Periode des stetigen Abschwungs an.

Die erste Phase von 1950 bis 1970 umfasst die Gründungsphase des Buchclubs. Von 1950 bis 1960 verzeichnete der Buchclub ein exponentielles Mitgliederwachstum. Innerhalb eines Jahres zählte der Club bereits 100.000 Mitglieder, ${ }^{619}$ im Jahr 1954 wurde erstmals die Millionengrenze erreicht. ${ }^{620}$ Der steile Mitgliederzuwachs setzte sich bis zum Jahr 1961 fort, als der Buchclub auf einem vorläufigen Höhepunkt nur elf Jahre nach seiner Gründung nahezu drei Millionen Mitglieder vertraglich bin- 
den konnte. ${ }^{621}$ Das Wachstum ließ sich jedoch nicht fortsetzen. In den folgenden zehn Jahren sank der Mitgliederbestand um ca. eine Million auf zwei Millionen ab. ${ }^{622}$ Wie anhand der historischen Entwicklung des Buchclubs in Kapitel 4.3.1.2 dargestellt, reagierte der Club mit Neugründungen kleinerer Buchgemeinschaften (deren Bestände meist später in den Lesering überführt wurden) sowie mit Übernahmen von zahlreichen Wettbewerbern.

Der Mitgliederschwund konnte schließlich mit Beginn der zweiten Phase im Jahr 1970 endgültig aufgehalten und in einen erneuten Zuwachs umgekehrt werden. Vor allem durch die Übernahme des Deutschen Bildungskreises im Jahr 1969, eine 50\%Beteiligung an der Deutschen Buchgemeinschaft (DBG) im Jahr 1970 sowie die Übernahme der Europäischen Bildungsgemeinschaft (EBG) im Jahr 1973 gelang es, den erodierenden Mitgliederbestand in eine absolute Zunahme umzukehren. ${ }^{623}$ Dass der resultierende Anstieg auf fünf Millionen Mitglieder im Jahr 1980624 zu nicht geringen Teilen auf der Integration übernommener Buchclubs basierte und nicht die Folge rein organischen Wachstums war, zeigten bereits Mitte der 70er Jahre festgestellte Marktsättigungserscheinungen deutlich an. ${ }^{625}$ Die Marktsättigung äußerte sich schließlich in einem erneuten Mitgliederrückgang von 1980 bis 1990, indem der Club innerhalb von zehn Jahren eine Million Kunden einbüßte und sein Bestand von fünf auf ca. vier Millionen erodierte. ${ }^{626}$

Ähnlich der Reaktion von 1970 bis 1980 wurde dem Mitgliederschwund in der dritten Phase mit der Übernahme konkurrierender Buchgemeinschaften begegnet. Im Jahr 1989 übernahm Bertelsmann die verbliebenen 50\% der Deutschen Buchgemein-

621 Vgl. BUA-1000/27 (Mitgliederentwicklung, 1982).

622 Vgl. BUA-1000/27 (Mitgliederentwicklung, 1982).

623 Vgl. Kapitel 4.3.1.3 dieser Arbeit.

624 Vgl. BUA-1000/27 (Mitgliederentwicklung, 1982).

625 Vgl. BG (1977/78), S. 12.

626 Vgl. Böttger, W. (2002, 11.09.). 
schaft (DBG), zwei Jahre später erfolgte eine Beteiligung zu 24,9\% an dem letzten großen Wettbewerber, dem Deutschen Bücherbund (DBB), dessen restliche Anteile nur ein Jahr später, im Jahr 1992, ebenfalls von Bertelsmann gekauft wurden. ${ }^{627}$ Führte allein die Mitgliederintegration durch die Übernahme des Deutschen Bücherbunds zu einer Bestandserhöhung von mehr als einer Million, so bedeutete die nahezu zeitgleich stattfindende Wiedervereinigung Deutschlands eine unerwartete Markterweiterung. Aufgrund des hohen Nachholbedarfs in der DDR vor allem an praktischer Literatur wie Ratgebern und Reiseführern gelang es Bertelsmann innerhalb von nur einem Jahr, eine weitere Million Mitglieder in den neuen Bundesländern zu gewinnen. Der Kauf des Deutschen Bücherbundes sowie die Markterweiterung durch die Wiedervereinigung konnten den Abwärtstrend der 80er Jahre aufhalten und in eine Bestandserweiterung auf sechs Millionen Mitglieder im Jahr 1992 umkehren. ${ }^{628}$ Nahezu so schnell, wie die zwei Millionen Mitglieder hinzugewonnen wurden, so schnell wurden diese in den folgenden fünf Jahren wieder verloren. Der Bestand von sechs Millionen Mitgliedern im Jahr 1992 sank auf vier Millionen im Jahr 1997. ${ }^{629}$ Dieser Abwärtstrend setzte sich gleichermaßen in den letzten zehn Jahren der Untersuchung fort. Von 1997 bis 2007 erodierte der Mitgliederbestand latent, aber stetig von vier auf drei Millionen und erreichte somit seinen vorläufigen Tiefstand. ${ }^{630}$

Nachfolgende Abbildung 21 verdeutlicht die Mitgliederentwicklung der Bertelsmann Buchclubs von 1950 bis 2007. Besonders deutlich werden hier die drei jeweils von Aufschwung und Rückgang gekennzeichneten Phasen von 1950 bis 1970, von 1970 bis 1990 sowie von 1990 bis $2007 . .^{631}$

627 Vgl. Kapitel 4.3.1.3 dieser Arbeit.

628 Vgl. Böttger, W. (2002, 11.09.).

${ }^{629}$ Vgl. Der Club Bertelsmann (2008).

${ }^{630}$ Vgl. Der Club Bertelsmann (2008).

631 Die Zahlen der Mitgliederentwicklung wurden aus folgenden Quellen zusammengetragen: BUA-1000/27 (Mitgliederentwicklung, 1982); Gööck, R. (1968); Der Club Bertelsmann 
Abbildung 21: Entwicklung des Mitgliedsbestands des Bertelsmann Buchclubs Deutschland Mitglieder in Mio.

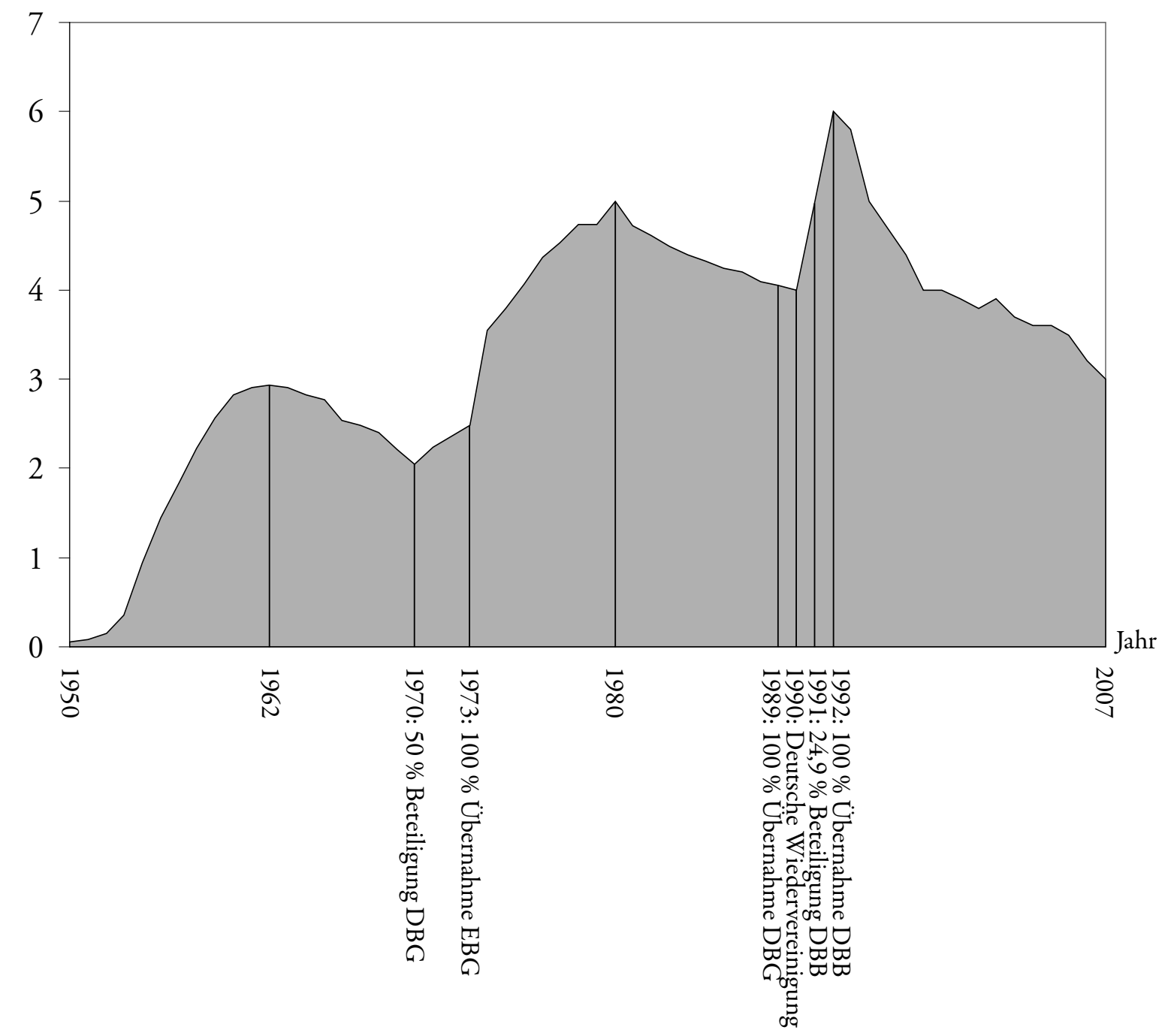

Quelle: Eigene Darstellung.

Gibt die Entwicklung des Mitgliederbestandes bereits ein verlässliches Abbild der wirtschaftlichen Entwicklung des Bertelsmann Buchclubs, so wurde das zusätzliche Ziel verfolgt, die einzelnen Entwicklungsphasen mit Umsatzzahlen zu untermauern. Bei deren Erhebung zeigten sich folgende Schwierigkeiten:

(2008); BUA-0006/15 (Bertelsmann Clubarbeit: Chronik, 1977); Bertelsmann AG (1985a); Böttger, W. (2002). Einschränkend muss gesagt werden, dass die Mitgliederzahlen für die Jahre 1986, 1988, 1989, 1991, 1992, 1993 und 1994 nicht vorlagen und die entsprechenden Zahlen auf Schätzungen das Autors beruhen. 
Wie bereits anhand der historischen Entwicklung des Buchclubs deutlich geworden ist, änderte sich mit steigender Unternehmensgröße die Rechtsform von Bertelsmann und der Clubgeschäfte mehrfach ( $\mathrm{GmbH}$, oHG, $\mathrm{AG})$. Analog hierzu erfolgten zahlreiche Reorganisationsprozesse, in deren Rahmen sich zugleich die bilanzielle Ausweisung der Club-Geschäftszahlen änderte. Vor allem die Zusammenfassung der Buchgeschäfte zu der Einheit ,Buch Deutschland, Österreich, Schweiz', zu der neben den Buchgemeinschaften auch die Geschäftsaktivitäten der Verlagsgruppe von Bertelsmann gehören, und die Einführung der ,Produktlinie Buch' im Jahr 1993 behindern die Transparenz einer getrennten Umsatzbetrachtung einzelner nationaler Buchgemeinschaften. Ein kontinuierlicher Vergleich der jährlichen Umsatzzahlen ließ sich aufgrund der unterschiedlichen Referenzgrößen somit nicht vornehmen. Bertelsmann selbst tätigte aus unternehmenspolitischen Gründen keine Aussagen zur konkreten Umsatz- bzw. Gewinnentwicklung. Die jüngsten Zahlen zur Gewinnentwicklung des Bertelsmann Buchclubs in Deutschland sind entsprechend der Tagespresse entnommen.

Es wird geschätzt, dass der deutsche Buchclub im Jahr 2002 einen Verlust von 52 Millionen, im Jahr 2003 von 15 Millionen und im Jahr 2004 von 10 Millionen Euro erwirtschaftet hat. Der kummulierte Verlust des deutschen Bertelsmann Buchclubs von 1999 bis 2006 wird auf mehr als 100 Millionen Euro geschätzt. ${ }^{632}$

Zusammenfassend lässt sich festhalten, dass der kontinuierliche Mitgliederverlust - kurzfristig aufgehalten durch zwei einmalige Sondereffekte Anfang der 90er Jahre die wirtschaftlichen Schwierigkeiten des Buchclubs bereits seit Beginn der 80er Jahre widerspiegelt. Anhand der spätestens seit Beginn der 90er Jahre aufgetretenen Verluste lässt sich erahnen, dass die Umsatzentwicklung einen ähnlichen Verlauf genommen hat, so dass bereits in den 80er Jahren von wirtschaftlicher Ineffizienz auszugehen ist. 
Angesichts der lang anhaltenden ökonomischen Schwierigkeiten soll nun in einem zweiten Schritt untersucht werden, ob im Buchclub Versuche unternommen worden sind, die strategische Trajektorie zu durchbrechen. Das Ziel dieses Schrittes ist zu prüfen, ob Immunität in Form von Wandlungswiderständen vorliegt.

\subsection{Immunität}

Zahlreiche Studien belegen, dass organisationale Trägheit durch das Aufbrechen verfestigter Strukturen in Form von intentionalen oder emergenten Störungen (z. B. externe Schocks) durchbrochen werden kann. Es zeigte sich, dass der personelle Austausch der Geschäftsführung und des Managementteams häufig mit einer Neuorientierung der strategischen Entscheidungspraxis einhergeht, da externe Geschäftsführer und neu zusammengesetzte Managementteams nicht den etablierten Mustern und Routinen der Organisation unterliegen. ${ }^{633}$ Sofern derartige Bemühungen, strategischen Wandel zu erzeugen und die Trägheit von Systemen zu durchbrechen, wirkungslos bleiben, ist aus Sicht der Pfadtheorie davon auszugehen, dass sich das System im $\mathrm{Zu}$ stand eines Lock-Ins befindet und somit immun gegenüber Wandlungseinflüssen ist.

Im Fall des Bertelsmann Buchclubs ließ sich der strategische Lock-In anhand des mehrfachen Austauschs der Geschäftsführung sowohl auf Club- als auch auf Konzernebene aufzeigen. Im Bertelsmann Buchclub wechselte allein von 1990 bis 2007 zehnmal die Geschäftsführung. So erfolgte in den Jahren 1990, 1992, 1993, 1994, 1995, 1997, 2001, 2003, 2004 und zuletzt Ende 2005 ein Austausch in der ClubFührungsspitze, der nicht selten mit einer personellen Neugliederung im gesamten Führungsteam einherging. ${ }^{634}$

633 Vgl. Huff, J.O. et al. (1992), S. 69f.; Lant, T.K./Milliken, F.J. (1992), S. 585-608; Horn, J.T. et al. (2006).

634 WIC (1994/01), S. 2; WIC (1995/10); WIC (1997/11); WIC (2001/02-03); WIC (2003/02); WIC (2004/10), S. 2; WIC (2005/06), S. 2. 
Auch die Vermutung, die Ursache der strategischen Trajektorie im Buchclub auf Konzernebene zu heben und durch den machtvollen Einfluss der Unternehmensgründer - allen voran Herrn Reinhard Mohn - erklären zu können, ließ sich nicht erhärten, da selbst auf Konzernebene mit dem mehrfachen Austausch des Vorstandsvorsitzes die unternehmerische Gesamtverantwortung in unterschiedliche Hände gelegt wurde. Auf Herrn Mohn, den gleichzeitigen Gründer des Bertelsmann Buchclubs, folgte im Jahr 1981 Herr Fischer, der von Herrn Wössner im Jahr 1983 abgelöst wurde. Schließlich übernahm Herr Middelhoff im Jahr 1998 den Vorstandsvorsitz, welchen er schließlich an Herrn Thielen im Jahr 2002 übergab. ${ }^{635}$ Die einzelnen Vorstände verliehen dem Gesamtunternehmen sehr unterschiedliche Impulse. Als prägnantes Beispiel sei die Ära unter Herrn Middelhoff angeführt, der die Bertelsmann AG und seine einzelnen Divisionen auf die Anforderungen des Multimedia-Zeitalters und der Internet-Technologie ausrichtete. ${ }^{636}$

Es lässt sich also festhalten, dass auch die mehrfache Irritation sowohl des Systems ,Bertelsmann AG’ als auch des Systems ,Buchclub' durch personelle Veränderungen keine nachhaltige Richtungsänderung der strategischen Trajektorie bewirkte. Im Gegenteil: Neue Ansätze (Best'Seller, Boulevard, BOL sowie die Öffnung hin zu einem Medienclub) wurden zugunsten der etablierten Geschäftsstrategie nach kurzer Zeit wieder aufgegeben.

Ob die beschriebene Wandlungsresistenz auf die Unkenntnis, das Unbewusstsein oder den Unwillen, alternative Strategieoptionen langfristig zu realisieren, zurückzuführen ist, soll im nun folgenden Kapitel zur Ignoranz als drittes Element erörtert werden.

${ }^{635}$ Vgl. Bertelsmann (1985), S. 404; Börsenblatt (1983, 23.12.), S. 2763; Der Spiegel (1998, 26.10.); FTD (2002, 29.07.), S. 1.

636 Vgl. Wirtschaftswoche $(1998,29.10)$; FTD $(2002,29.07)$, S. 1. 


\subsection{Ignoranz}

Beschreibt die Immunität der Entscheidungstrajektorie eine Wandlungsresistenz und deutet so bereits auf die Sogwirkung des Lock-Ins hin, so ist mit Ignoranz die unbewusste (,nicht wissen' und ,nicht beachten') bzw. bewusste (,nicht wissen wollen') Vernachlässigung alternativer Handlungsmöglichkeiten gemeint.

Marktsättigungstendenzen im Segment der Buchgemeinschaften zeichneten sich bereits in den 70er Jahren ab. Als Reaktion erfolgte die Übertragung der Clubidee in ausländische, noch nicht gesättigte Märkte, ohne dem deutschen Buchclub eine zukunftsfähige strategische Neuorientierung zu verleihen. Stattdessen wurde sinkenden Mitgliederzahlen mit der Übernahme von Wettbewerbern begegnet und das etablierte Geschäftsmodell weiterhin fortgesetzt. Ignoriert wurde die Tatsache, dass selbst die marktbeherrschende Stellung im Segment der Buchgemeinschaften keine nachhaltige Sicherung wirtschaftlichen Erfolgs bedeutete, da sich die Wettbewerbsverhältnisse im Buchmarkt grundlegend änderten. Auch kritische Stimmen in den 80er Jahren prognostizierten wirtschaftliche Schwierigkeiten und benannten die Notwendigkeit strategischer Änderungen, dennoch blieben diese ohne Auswirkungen auf den strategischen Entscheidungsverlauf. ${ }^{637}$ Unter Verweis auf die nahezu monopolistische Stellung im Markt der Buchgemeinschaften wurde die Notwendigkeit zum Wandel bewusst ignoriert (,nicht wissen wollen). ${ }^{638}$

Diese Einstellung änderte sich in den 90er Jahren, als die Mitgliedererosion durch Übernahmen und Beteiligungen nicht mehr aufzuhalten war. Fortan wurde zwar

637 „Ich erinnere mich, dass damals [...] der Vorstandsvorsitzende, [...] schon den starken Eindruck hatte, man muss am Buchclub heftigste Renovierungsarbeiten machen." Interview BME07969 (2007, 16.04.), S. 4.; „Also, im Club gibt es eigentlich eine erstaunliche Hellsicht. Also, es gibt Papiere von Anfang der 80er Jahre, wo sehr eloquent dargelegt wird, warum das Clubgeschäft wie wir es kennen mausetot ist und keine Zukunft hat." Interview BME-06021 (2006, 21.12.), S. 9.

638 „Man] hielt das für Faxen, nach dem Motto: Wir haben ja das Monopol, es gibt ja keinen anderen Buchclub oder keinen wesentlichen anderen.“ Interview BME-07969 (2007, 16.04.), S. 4. 
prinzipiell Änderungsbedarf wahrgenommen und formuliert, jedoch nicht in langfristig wirksame Strategiealternativen umgesetzt. Innovative Strategievarianten wie der Aufbau von, Wohlfühlbuchhandlungen' (Boulevard), Beitragsfreiheit (Best'Seller) oder Online-Handel (BOL) wurden lediglich als Reaktionen auf bereits bestehende Wettbewerber eingeführt, so dass die jeweiligen Marktsegmente bereits besetzt waren: „Wenn man eine Antwort gibt, hat ein anderer schon was gesagt, oder - wie in diesem Fall [Boulevard] - getan. Man [Bertelsmann] ist also ein Stück weit reaktiv und nicht proaktiv. “639 Möglichkeiten des proaktiven Handlungsbedarfs wurden nicht gesehen bzw. nicht umgesetzt (,nicht beachten').

Es lässt sich schlussfolgern, dass die anfängliche Ignorierung der Änderungsnotwendigkeit mit Realisierung der anhaltenden ökonomischen Schwierigkeiten in eine andere Form von Ignoranz überging. Während bis Anfang der 90er Jahre alternative strategische Wege bewusst ignoriert wurden, bewirkten die Trägheitskräfte der strategischen Trajektorie eine zunehmende Unmöglichkeit, alternative Strategieoptionen wahrzunehmen.

Lassen die drei Elemente der Ineffizienz, Immunität und Ignoranz bereits auf einen pfadbedingten Lock-In schließen, so soll im nun folgenden Kapitel ergänzend dargestellt werden, welche Faktoren stabilisierend auf die strategische Entscheidungstrajektorie eingewirkt haben.

\subsection{Stabilisierende Faktoren}

Stabilisierende Faktoren bekräftigen die strategische Rigidität, gelten jedoch nicht als alleinige Ursache zur Konstitution von Pfadabhängigkeiten, da sie keine Prozessdynamik entwickeln. Vielmehr zeichnen sie sich durch eine zeitlich stabile Wirkkraft aus, durch welche sie strategische Beharrungstendenzen verfestigen, ohne jedoch durch die strategischen Entscheidungen des rigiden, pfadabhängigen Systems rück- 
wirkend beeinflusst werden zu können. Stabilisierende Faktoren sind somit von Selbstverstärkungsmechanismen zu unterscheiden, die zwar laut pfadtheoretischen Annahmen zum Zeitpunkt eines Lock-Ins nicht mehr gegenseitig verstärkend wirken müssen, jedoch nach wie vor ein zirkulär-rekursives Verhältnis beschreiben. Anders die stabilisierenden Faktoren: Diese wirken gerade nicht zirkulär, sondern sind einseitig, linear ausgerichtet.

Als stabilisierende Faktoren konnten im Fall des Bertelsmann Buchclubs die Eigentümerstruktur des Bertelsmann Konzerns, die ausländischen Bertelsmann Buchclubs als Benchmarks sowie die Preisbindung im deutschen Buchmarkt ausgemacht werden. Alle drei Faktoren sollen in den nun folgenden Abschnitten erläutert werden.

\section{Konzern- und Eigentümerstruktur}

Wie im Rahmen der historischen Entwicklung des Bertelsmann Konzerns bereits deutlich wurde, steht in der vorliegenden Arbeit zwar die strategische Entwicklung des Clubs als fokale Untersuchungseinheit im Mittelpunkt, ohne allerdings deren historische Einbettung in den Konzernverbund außer Acht zu lassen.

Erst der Club Bertelsmann, hinlänglich als „Königsidee“640 bezeichnet, ermöglichte die Finanzierung und den Aufbau des multinationalen Medienkonzerns Bertelsmann AG. Ohne die hohen Gewinne aus dem Buchclubgeschäft wäre eine Ausweitung der medialen Geschäftstätigkeiten von Bertelsmann kaum möglich gewesen, so dass der Buchclub auch häufig als ,Keimzelle’ des Konzerns betrachtet wird. ${ }^{641}$ Die Tatsache, dass der bis heute bestehende Konzern zu den Wurzeln des Buchclubs zurückführt, räumt selbigem eine historische Sonderstellung im Bertelsmann Firmenverbund ein,

640 Tietz, B. (1985), S. 339.

${ }^{641}$ „Der Club hat alles möglich gemacht. Das Geld des Clubs hat alles möglich gemacht.“ Interview BME-07010 (2007, 12.03.), S. 10. 
auf die sich in Teilen die strategischen Beharrungstendenzen zurückführen lassen. ${ }^{642}$ So genießt der Club einen besonderen, auf der eigenen Unternehmenshistorie basierenden Schutz, der ihm einerseits innerkonzernliche Unterstützung zusichert, andererseits jedoch auch stabilisierend auf die strategische Kontinuität einwirkt. ${ }^{643}$

Die Analyseergebnisse zeigten, dass hierfür die Eigentümerstruktur des Bertelsmann Konzerns eine wesentliche Rolle spielt. Einer der beiden zentralen Gründungsväter des Buchclubs, Reinhard Mohn, ${ }^{644}$ und seine Familie kontrollierten auch noch im Jahr 2007 die Geschäftsaktivitäten des Konzerns. ${ }^{645}$ Obwohl das operative Geschäft von externen Managern eigenverantwortlich gestaltet und die Club-Geschäftsführung im Jahr 2005 von Gütersloh, dem Sitz der Konzernzentrale, nach Berlin verlegt wurde, ließ sich ein starker, emotional geprägter Gründungseinfluss über den gesamten Analysezeitraum beobachten: ${ }^{446}$ „'Der deutsche Klub hat noch immer eine emotionale Bedeutung für den Konzern', weiß Walgenbach [Vorstand Direct Group]. Schließlich erwirtschaftete Reinhard Mohn mit diesem in den 50er und 60er Jahren hoch rentablen Geschäft das Geld für die spätere Expansion. “ ${ }^{647}$

Von einer deterministischen Einflussnahme auf die strategische Entwicklung des Buchclubs muss dennoch abgesehen werden, da im Verlauf der Club-Historie Ent-

${ }^{642}$ „Wäre [der Club] ein anderes Geschäft dieser Art, nehmen wir mal an, es wäre ein Stadtplangeschäft gewesen, oder ein sagen wir mal ein CD-Presswerk in Russland, dann wäre das längst weg. Also wäre es wahrscheinlich abgestoßen worden, mit einem Wettbewerber fusioniert, wie auch immer.“ Interview BME-06021 (2006, 21.12.), S. 11.

643 „Wenn das nicht sozusagen die Keimzelle dessen wäre, woraus Bertelsmann nach dem 2. Weltkriege hervorgegangen ist, also wenn Bertelsmann maßgeblich Blackstone oder einer anderen Private Company, Equity Company, gehören würde, wäre der Buchclub schon weg." Interview BME-07969 (2007, 16.04.), S. 6.

644 Zusammen mit dem Vertriebsleiter Fritz Wixforth baute Reinhard Mohn das Clubgeschäft 1950 auf. Vgl. Gööck, R. (1968).

645 „Bewusst hat er [Reinhard Mohn] die Entscheidungshoheit schrittweise an seine [...] Frau abgegeben. Bei wichtigen Themen rede er zwar noch mit. ,Aber Liz nimmt stark Einfluss auf seine Entscheidung und gibt die Richtung vor', sagt ein früherer Bertelsmann-Manager." FAZ (2006, 27.06.), S. 16.

646 Vgl. Die Welt $(1986,26.06.) ; \mathrm{W} \& \mathrm{~V}(2005,15.09)$, S. 30.

${ }^{647}$ Handelsblatt $(2006,26.09$.), S. 10. 
scheidungen getroffen wurden, die im klaren Gegensatz zum Gründungsanspruch des Inhabers standen. Aussagen des Gründungsvaters Reinhard Mohn „So lange ich diesen Laden führe, so lange ich verantwortlich bin, wird an dieser Königsidee [dem Buchclub] nichts geändert." ${ }^{468}$ oder „Ich verspreche, solange ich lebe, wird der Konzern sich nicht buchhändlerisch betätigen. “649 kollidieren mit strategischen Entwicklungen ab Mitte der 90er Jahre, als mit dem Boulevard-Konzept durchaus probiert wurde, eine Buchhandelskette aufzubauen und sich somit buchhändlerisch zu betätigen. Ähnlich sind die in 2006 laut gewordenen Mutmaßungen der Presse zu deuten, dass Bertelsmann mehrfach versuchte, den Buchclub mit der einflussreichen Buchhandelskette Thalia zu fusionieren. ${ }^{650}$ Dass die Fusion schließlich nicht erfolgte, scheint eher gescheiterten Verhandlungen als dem Unwillen von Bertelsmann, sich doch buchhändlerisch zu betätigen, Rechnung zu tragen. ${ }^{651}$

Zusammenfassend lässt sich festhalten, dass der Gründungseinfluss und die Eigentümerstruktur von Bertelsmann eine nicht unwesentliche Rolle in der Strategieentwicklung des Buchclubs spielen. Eine alleinige Erklärung der strategischen Rigidität durch die machtvolle Einflussnahme zentraler Gründungsfiguren würde jedoch zu kurz greifen.

\section{Ausländische Clubs als Benchmarks}

Ein zweiter, die Clubstrategie stabilisierender Faktor liegt in der erfolgreichen Entwicklung ausländischer Buchgemeinschaften von Bertelsmann, die gleichermaßen als Vorbild und Legitimation für die deutschen Clubaktivitäten gelten. Wie in Kapitel 4.3.1 ausführlich erläutert, begann 1962 die bis heute anhaltende Expansionswelle

648 Interview BE-07033 (2007, 17.07.), S. 2.

649 Interview BE-07033 (2007, 17.07.), S. 30.

650 Vgl. SZ (2006, 26.07.); Die Welt (2006, 07.10.).

651 Eine engere Verzahnung von Buchclub und Buchhandel wurde angestrebt. Vgl. SZ (2006, 26.09.), S. 17. 
der Buchclubidee ins inner- und außereuropäische Ausland. Besonders hervorzuheben sind die Buchgemeinschaften Círculo de Lectores in Spanien sowie France Loisirs in Frankreich, auf die im Zusammenhang mit den deutschen Clubgeschäften häufig verwiesen wird. ${ }^{652}$ Die ausländischen Bertelsmann Buchclubs wurden nach deutschem Vorbild gegründet und wiesen in ihrer strategischen Ausrichtung eine hohe Kongruenz zum deutschen Prototyp auf: „Wenn jemand einen Club versteht, kann er alle Clubs verstehen, weil die Logik des Vorgehens ist dieselbe. "653

Auch wenn die jeweilige Ausgestaltung den nationalen Gegebenheiten angepasst wurde (z. B. werden die Bücher in Spanien per Boten ausgeliefert, der auch neue Bestellungen aufnimmt $\left.{ }^{654}\right)$, ist es „bis zum heutigen Tag [...] so, dass eigentlich alle grundsätzlichen Dinge, die in Deutschland funktionieren auch in Frankreich funktionieren und umgekehrt, also es ist zwischen Deutschland und Frankreich ein extrem reger Ideenaustausch." “655 In diesem Sinne werden gerade die Bertelsmann Buchclubs in Frankreich und Spanien - beide arbeiten sehr erfolgreich und rentabel - als Referenzgröße für die Durchsetzbarkeit des Geschäftsmodells ,Buchgemeinschaft’ herangezogen. ${ }^{656}$ Auch France Loisirs durchlebte Phasen wirtschaftlichen Abschwungs, die schließlich durch Restrukturierungsmaßnahmen abgewendet und in einen langfristigen Aufwärtstrend umgewandelt werden konnten. Ähnliches gilt für Círculo de Lectores in Spanien - diejenige Buchgemeinschaft, welche in Spanien einen Status als Kulturinstitution erreichen konnte und mit dem spanischen Königspaar als Ehrenmitgliedern wirbt.

652 Vgl. Handelsblatt $(2004,16.12)$, S. 14.

653 Interview BME-06021 (2006, 21.12.), S. 12.

654 Vgl. Interview BME-07963 (2007, 24.04.), S. 9.

655 Interview BME-06021 (2006, 21.12.), S. 12.

656 Vgl. SZ (2006, 26.09.), S. 17; W\&V (2005, 28.04.), S. 10. „Dass man mit den Buchclubs Geld verdienen kann, hätten die Geschäfte in Frankreich und Spanien bewiesen. "Börsenblatt Online (2006, 23.08.). 
Ökonomischer sowie Image-Erfolg der ausländischen Buchclubs strahlten so bereits seit den 80er Jahren auf die Kontinuität der strategischen Entscheidungen im deutschen Clubgeschäft aus. Die erfolgreichen Vorbilder aus Frankreich und Spanien rechtfertigten die Konstanz der deutschen Clubstrategien, so dass von Jahr zu Jahr gehofft wurde, durch kleinere Kursänderungen (Renovierung der Clubfilialen, Relaunch des Clubkatalogs $\left.{ }^{657}\right)$, mit der bisherigen Strategie zu den Erfolgen in Frankreich und Spanien aufschließen zu können. ${ }^{658}$

Nicht thematisiert wird dabei häufig, dass in den drei Ländern eine sehr unterschiedliche Wettbewerbssituation herrscht: „In Spanien ist der Wettbewerb am geringsten, in Frankreich so mittel und in Deutschland am höchsten. “659

\section{Preisbindung im deutschen Buchmarkt}

Ein dritter zentraler Faktor, der stabilisierend auf die strategische Kontinuität des Buchclubs einwirkte, ist die gesetzliche Buchpreisbindung in Deutschland.

Wie ausführlich in den Kapiteln 4.1.3 und 4.4.2.2.4 erläutert, ist der feste Verkaufspreis für sämtliche Buchhändler bindend. Einzige Ausnahme bilden die Buchgemeinschaften, die auf Basis ihres Mitgliedschaftssystems Parallelausgaben in unterschiedlicher Aufmachung und mit zeitlichem Abstand zum Erscheinen im regulären Buchhandel herausbringen dürfen. Die Gründung einer Buchgemeinschaft mit Abonnement war somit für Bertelsmann die einzige Lösung, Bücher in relativer Zeitnähe zum Ersterscheinungstermin zu vergünstigten Preisen anzubieten. ${ }^{660}$

657 Vgl. Handelsblatt $(2003,18.09$.$) , S. 17$.

658 Vgl. Handelsblatt $(2004,16.12$.), S. 14.

659 Interview BME-06021 (2006, 21.12.), S. 3.

660 „Die Erlaubnis, sage ich mal, oder die Möglichkeit, trotz der allgemeinen Preisbindung, Bücher preiswerter anzubieten, findet ja seine einzige rechtliche Begründung in der Tatsache, dass der Käufer Mitglied in einem Club ist, in dem er sich zu bestimmten Dingen verpflichtet." Interview BME-07969 (2007, 16.04.), S. 5. 
Auch wenn die Buchpreisbindung im Laufe des Analysezeitraums kontinuierlich diskutiert und Abstandsfristen verkürzt wurden, so hat sie mit ihrer gesetzlichen Verbindlichkeit bis heute Bestand und beeinflusst somit mittelbar auch die strategische Entwicklung des Buchclubs. So wirbt der Club bei einer Vielzahl seiner im Programm befindlichen Buchtitel mit der direkten Gegenüberstellung von Buchhandelspreis und dem verbilligten Preis der Clubausgabe. ${ }^{601}$ Diese preisliche Differenzierung wäre ohne Mitgliedschaftssystem nicht möglich, da allein die Abonnementsverpflichtung den Club berechtigt, seine Bücher nach Einhaltung der Abstandsfrist vergünstigt anzubieten. Die Abschaffung der verpflichtenden Mitgliedschaft im Buchclub - der als solcher fortan keinen Clubcharakter im engeren Sinn mehr hätte - wäre zwar prinzipiell möglich, allerdings müsste gleichermaßen die zeitnahe Herausgabe von Parallel(lizenz)ausgaben aufgegeben werden, die nach wie vor zum Kern des Clubgeschäfts zählt.

Die feste Buchpreisbindung in Deutschland birgt für den Club in diesem Sinne zu gleichen Teilen Vor- und Nachteile. Auf der einen Seite ermöglicht die Buchpreisbindung erst die Preisdifferenzierung vom regulären Buchhandel und somit den für die Kunden preisgünstigen Buchvertrieb, auf der anderen Seite verhindert sie die potentielle Modifikation des Clubcharakters.

Die Buchpreisbindung wirkt somit - wenn auch nicht gegenseitig verstärkend, weil der strategische Verlauf des Buchclubs nicht auf die Preisgesetzgebung rückwirkt - stabilisierend auf die Strategieentwicklung des Clubs ein.

\subsection{Zwischenfazit zum Lock-In}

In Kapitel 4.4.4.4 sollte untersucht werden, ob die strategische Rigidität des Bertelsmann Buchclubs durch einen Lock-In spezifiziert werden kann. Hierzu wurde die 
strategische Entwicklung auf die Elemente Ineffizienz, Immunität, Ignoranz sowie stabilisierende Faktoren hin untersucht.

Es zeigte sich, dass der Club bereits Anfang der 80er Jahre massive Mitgliederrückgänge erlitten hat und spätestens seit Anfang der 90er Jahre unrentabel wurde, so dass sich der Verdacht auf wirtschaftliche Ineffizienz bestätigte.

Zusätzlich erwies sich der strategische Entscheidungsverlauf als resistent gegenüber externer Einflussnahme in Form von zahlreichen Managementwechseln sowohl auf Buchclub- als auch auf Konzernebene, so dass sich ebenfalls die Vermutung auf Immunität des Buchclubs gegenüber externer Einflussnahme erhärtete.

Ignoranz ließ sich in zwei unterschiedlichen Formen feststellen. Bis Mitte der 80er Jahre zeigte sich zunächst der Unwille, strategischen Wandel einzuleiten. Angesichts der lang anhaltenden wirtschaftlichen Schwierigkeiten, ließ sich in den 90er Jahren erstmals die Bereitschaft einer strategischen Neuorientierung beobachten. Die Implementierung strategischer Varianten erfolgte jedoch ausnahmslos in Reaktion auf strategische Schritte der Wettbewerber. Möglichkeiten der proaktiven strategischen Neugestaltung wurden nicht wahrgenommen.

Schließlich konnten ein nachhaltiger Gründungseinfluss, erfolgreiche ausländische Buchclubs als Benchmarks sowie die Buchpreisbindung in Deutschland als stabilisierende Faktoren ermittelt werden, welche die strategische Rigidität zusätzlich verfestigten.

Zusammenfassend bestätigte sich somit der Verdacht auf einen strategischen Lock-In. Die Ergebnisse lassen den Schluss zu, dass das Unternehmen nur schwer in der Lage ist, die strategische Rigidität zu durchbrechen.

\subsubsection{Zusammenfassung und Einordnung in das Drei-Phasen-Modell der Pfadentstehung}

In Anlehnung an den zweistufigen Aufbau der Pfadanalyse wies Schritt 1 strategische Rigidität im Fall des Bertelsmann Buchclubs nach. Die strategische Rigidität zeigte 
sich anhand der strategischen Kontinuität des Clubs trotz hoher Umweltdynamik und Wettbewerbszunahme im deutschen Buchmarkt seit den 80er Jahren.

An den Rigiditäts-Befund schloss sich in Schritt 2 die Pfaddiagnose an. Hierzu wurden die auf den zentralen Annahmen der Pfadtheorie basierenden Komponenten der Historizität, des Critical Junctures, der positiven Rückkopplung sowie des LockIns erhoben. Sämtliche Komponenten konnten in der strategischen Entwicklung des Buchclubs identifiziert werden und ließen somit den Schluss auf einen pfadabhängigen Strategieverlauf zu.

In den nachfolgenden Kapiteln gilt es nun, die einzelnen Komponenten in das Drei-Phasen-Modell der Pfadentstehung zu übertragen, um in chronologischer Zeitfolge die jeweiligen Stationen der Pfadentstehung bis hin zur Pfadabhängigkeit nachvollziehen zu können.

\subsubsection{Phase I: Historizität}

Zur Einordnung der strategischen Entwicklung der Buchclubs in Phase I des DreiPhasen-Modells der Pfadentstehung muss bereits auf die Jahre weit vor der Gründung des Clubs vor und während des Zweiten Weltkriegs zurückgegriffen werden. Der Grund hierfür liegt in der Tradition des bereits 1835 gegründeten C. Bertelsmann Verlags, dessen historische Vorprägung - auch wenn die Idee des Leserings noch nicht geboren worden war - auf den sich anschließenden strategischen Entscheidungsprozess im Buchclub ausstrahlte. Bevor Reinhard Mohn die Verlagsleitung 1947 übernahm, konnte das Verlagshaus Bertelsmann bereits auf erste Erfolge mit der Massenherstellung belletristischer Literatur zurückblicken. Hierzu zählten vor allem die ,Volksausgaben’ - vergünstigte Nachdrucke von klassischen Buchtiteln - sowie die „Feldpostausgaben', mit denen die Frontsoldaten während des Zweiten Weltkriegs beliefert wurden. 
Die damalige Geschäftsstrategie, günstige Sonderausgaben schöngeistiger Romane in großen Auflagen zu vertreiben, lässt bereits starke Analogien zu der späteren Geschäftsidee der Buchgemeinschaft erkennen. Diese historische Vorprägung ist jedoch keinesfalls deterministisch zu beurteilen, da der strategische Entscheidungsspielraum bis zur Gründung des Buchclubs noch sehr offen war.

\subsubsection{Phase II: Critical Juncture und Effekte der Selbstverstärkung}

Den Übergang von Phase I zu Phase II des dreigliedrigen Phasenmodells beschreibt das sog. Critical Juncture, das als analytischer Startpunkt des (positiven) Rückkopplungsmechanismus gilt. Für den Bertelsmann Buchclub bedeutete das kritische ,Small' zugleich ein ,Big Event’' die Buchclubgründung.

Mit der Gründung des Leserings im Jahr 1950 wurden die grundlegenden strategischen Weichen für den weiteren Entwicklungsverlauf gestellt. Zugleich bedeutete die Buchclubkonstitution, an der sich fortan sämtliche Geschäftsaktivitäten ausrichteten, einen jähen Bruch im historischen Verlauf des Verlagshauses Bertelsmann. Wie beschrieben, wäre vor der Buchclubgründung noch ein breites Spektrum an alternativen Strategieverläufen möglich gewesen. Bertelsmann hätte sich auf seine Tradition der theologischen Literatur rückbesinnen, den Weg einer rein verlegerischen Tätigkeit einschlagen oder sich z. B. auch als vertreibender Buchhändler betätigen können. Die Entscheidung, eine zweistufige Buchgemeinschaft ins Leben zu rufen, war somit trotz historischer Vorprägung (Phase I) eine Möglichkeit unter vielen, die jedoch rückblickend den strategischen Entscheidungsverlauf nachhaltig beeinflusst hat.

In den nachfolgenden Jahren - zunächst von 1950 bis 1960 - entfalteten sich die vollen Wirkkräfte der in Kapitel 4.4.4.3.2 vorgestellten Erfolgsspirale. Die strategischen Entscheidungen der Gründung bestätigten sich im Zusammenspiel mit Mitglieder- und Umsatzanstieg, Risikominimierung, steigender, verplanter Kaufkraft und 
Auflagenhöhe sowie den dadurch zu generierenden steigenden Skalenerträgen in einem kontinuierlichen Feedbackprozess selbst.

Parallel setzten sich - ebenfalls auf Basis von positiver Rückkopplung - Komplementaritäts- und Lerneffekte frei. In einem co-evolutorischen Prozess entstanden ana$\log$ zur Ausweitung der Clubaktivitäten Druckerei- und Logistikbetriebe sowie Eigenverlage um den Club herum. Durch die Expansion der Produktions- und Logistikkapazitäten ermöglichten diese ,Zuliefererbetriebe’ die weitere Vergrößerung des Clubs, der im Gegenzug mit seiner steigenden Auftragsvergabe deren Existenz und weiteren Ausbau sicherte.

Einen ähnlichen Beitrag zum Cluberfolg leisteten die fremden Vertriebsfirmen und Verpächter in ihrer Funktion der Werbung und Betreuung von Clubmitgliedern. Ohne die Werbe- und Vertriebsmacht des bestehenden Buchhandelsnetzes hätte der Club die Größenvorteile der Anfangsjahre nicht annähernd erreichen können. Diese wiederum erfreuten sich steigender Provisionszahlungen durch sichere Buchclubumsätze.

Parallel bildeten sich Lerneffekte in Form von Ergebniserwartungen aus. Steigende Umsätze und hohe Gewinnmargen äußerten sich in steigenden Erwartungen an selbige, die wiederum durch den Erfolg der Clubgeschäftstätigkeiten rückbestätigt wurden.

Steigende Skalenerträge, Komplementaritäts- sowie Lerneffekte bildeten so das Fundament für die zunächst als rational zu beurteilende Kontinuität im strategischen Entscheidungsprozess. Erstmals 1961 sanken die Mitgliederzahlen zunächst latent. Als Reaktion verleibte sich Bertelsmann in den Jahren von 1970 bis 1992 sukzessive nahezu sämtliche konkurrierenden Buchgemeinschaften ein und überführte einen Großteil deren Mitglieder in den Bestand des eigenen Buchclubs. Zusammen mit dem einmaligen Sondereffekt der Wiedervereinigung und der damit einhergehenden Markterweiterung gelang es Bertelsmann, den Markt der Buchgemeinschaften nahezu vollständig zu durchdringen und die Wirkkräfte der Erfolgsspirale zu verlängern. Vor allem der Erfolg durch das zeitliche Zusammentreffen von der Übernahme des letzten großen 
Wettbewerbers, des Deutschen Bücherbundes, und der Eroberung des ostdeutschen Marktes dürften die Lerneffekte weiter verstärkt und die Annahme von Richtigkeit des kontinuierlichen Strategieverlaufs bestätigt haben.

\subsubsection{Phase III: Lock-In, verstärkende und stabilisierende Effekte}

$\mathrm{Zu}$ welchem genauen Zeitpunkt der Übergang von Phase II zu Phase III erfolgte, ist aus einer rückblickenden Perspektive schwer zu ermitteln. Der Grund hierfür liegt in der Natur von pfadabhängigen Prozessen, deren Entstehung oftmals im Verborgenen abläuft, da kontinuierliche strategische Entscheidungsprozesse durchaus in Einklang mit ökonomischer Rationalität stehen können. Für den Beobachter - sowie für das handelnde System selbst - werden strategische Pfadprozesse spätestens dann bewusst, wenn eine Änderungsnotwendigkeit vorliegt, der (strategische) Handlungsspielraum jedoch schon so weit eingeschränkt ist, dass Anpassungen und Wandel nur noch schwer möglich sind. Vor diesem Hintergrund lässt sich der Übergang zu Phase III, die durch einen strategischen Lock-In geprägt ist, im Fall des Buchclubs spätestens auf Mitte der 90er Jahre terminieren, da zu diesem Zeitpunkt die Mitgliedererosion durch die endgültige Marktsättigung nicht mehr aufzuhalten war.

Die 90er Jahre stellen jedoch lediglich den spätesten Zeitpunkt eines Lock-Ins dar. Eine Vielzahl von Indizien deutet darauf hin, dass der strategische Handlungsspielraum bereits zu einem früheren Zeitpunkt entscheidend eingeschränkt war. Bereits Mitte der 70er Jahre wurde die Gefahr erkannt, dass sich der Buchclub durch Substitutionsprodukte und zunehmenden Wettbewerb auf dem Buchmarkt erschwerten Bedingungen auszusetzen hatte. Anstatt strategische Flexibilität durch die nachhaltige Entwicklung optionaler Geschäftsmodelle - wie die Stärkung einer rein buchhändlerischen Tätigkeit oder später die Fokussierung auf den Online-Handel - zu generieren, ließ sich neben einer Vielzahl von Tests und Experimenten keine langfristig entwickelte strategische Variante erkennen. 
Deutlich wird der frühzeitig eingeschränkte strategische Handlungsspielraum des Buchclubs im Vergleich zu dessen zentralen Wettbewerbern. Hugendubel eröffnete 1979 bereits seine erste großflächige, kundenfreundliche Buchhandlung. Thalia folgte fünf Jahre später im Jahr 1984. Neben der aufkommenden Konkurrenz im stationären Buchhandel entwickelte der Weltbild Verlag im Jahr 1987 ein dem Buchclub ähnliches Geschäftsmodell ohne Mitgliedschaftsverpflichtung und positionierte sich im Marktsegment der Buchversender, jedoch mit dem klaren Wettbewerbsvorteil der fehlenden Kaufverpflichtung. Schließlich drang Amazon.com durch die Übernahme des deutschen Online-Buchhändlers ABC-Bücherdienst unter dem Namen Amazon.de in den deutschen Online-Buchhandel ein. ${ }^{662}$

Der Buchclub, gestützt durch das Know-How und die Ertragskraft des ihn umgebenen Konzerns sowie die eigene langjährige Erfahrung im Buchmarkt, hätte sicherlich die Chance gehabt, sich selbst in den Marktsegmenten der großflächigen Buchhandlungen, freien Versender bzw. Online-Händler zu etablieren, setzte dies jedoch nicht um. Die Tatsache, dass mit den Strategieentwicklungen von Best!'Seller als Reaktion auf Weltbild, Boulevard als Gegenpol zu Hugendubel und Thalia sowie BOL als Antwort auf Amazon.de durchaus strategische Akzente auf den wachsenden Wettbewerb gesetzt wurden, lässt auf die Einsicht schließen, strategischen Wandel herbeiführen zu wollen. Dies erfolgte jedoch mit so starker zeitlicher Verzögerung, dass die jeweiligen Marktsegmente bereits längst besetzt waren. Durch die Einschränkung des Handlungs- bzw. Aktionsspielraums erzielten die strategischen Reaktionen keine nachhaltige Wirkung und liefen zumeist ins Leere. Die starke Einschränkung des Handlungsspielraums bereits Mitte der 80er Jahre lässt folglich darauf schließen, dass schon zu diesem Zeitpunkt der Prozess der Pfadentwicklung zu einer Abhängigkeit 
hin fortgeschritten war. Es lässt sich also vermuten, dass sich der strategische Lock-In in dem Zeitraum von Mitte der 80er bis Mitte der 90er Jahre eingesellt hat.

Durch die Mitgliedererosion von ca. sechs auf drei Millionen von 1992 bis 2007 sowie langjährige Verluste ist ab Mitte der 90er Jahre von sinkenden Skalenerträgen auszugehen. Die Komplementaritäts- und Lerneffekte wirken jedoch auch im Zustand des Lock-Ins nach. Aufgrund der co-evolutorischen, gegenseitig verstärkenden Entwicklung von Logistik, Druckereien und Verlagen bestehen bis heute komplementäre Geschäftsbeziehungen, die den strategischen Handlungsspielraum nach wie vor wesentlich einschränken und grundlegende strategische Änderungen erschweren. Hinzu kommen die Verpflichtungen gegenüber den Verpächterorganisationen, die durch ihren hohen Einfluss und das Mitspracherecht ebenfalls die strategische Variabilität des Buchclubs limitieren. Die Gewöhnung an hohe Gewinnspannen führte zu rigiden Ergebniserwartungen, die gleichermaßen eine Abweichung vom Kerngeschäft auf ein Minimum einschränkten und die Bandbreite des strategischen Aktionsradius radikal reduzierten.

Analog zu den verstärkenden Faktoren von Komplementaritäts- und Lerneffekten wirkten zudem noch stabilisierende Kräfte auf die strategische Kontinuität des Buchclubs ein. Die Rolle als Keimzelle des heutigen Konzerns sowie die noch immer dominierende familiengeführte Eigentümerstruktur von Bertelsmann beeinflussen gleichermaßen unterstützend und stabilisierend die strategische Kontinuität des Buchclubs. Der Erfolg der ausländischen Bertelsmann Buchclubs wird als Nachweis für das zeitgemäße Funktionieren und die Durchsetzungsfähigkeit des Geschäftsmodells „Buchclub' interpretiert und legitimiert somit die strategische Persistenz in Deutschland. Schließlich bestärkt die gesetzliche Buchpreisbindung die strategischen Säulen des Buchclubs, da nur so die preisliche Differenzierung vom regulären Buchhandel durchzuhalten ist. 
Folgende Abbildung 22 illustriert die einzelnen Entwicklungsstufen des Bertelsmann Buchclubs im Zeitverlauf:

Abbildung 22: Drei-Phasen-Modell der Pfadentstehung am Beispiel des Buchclubs

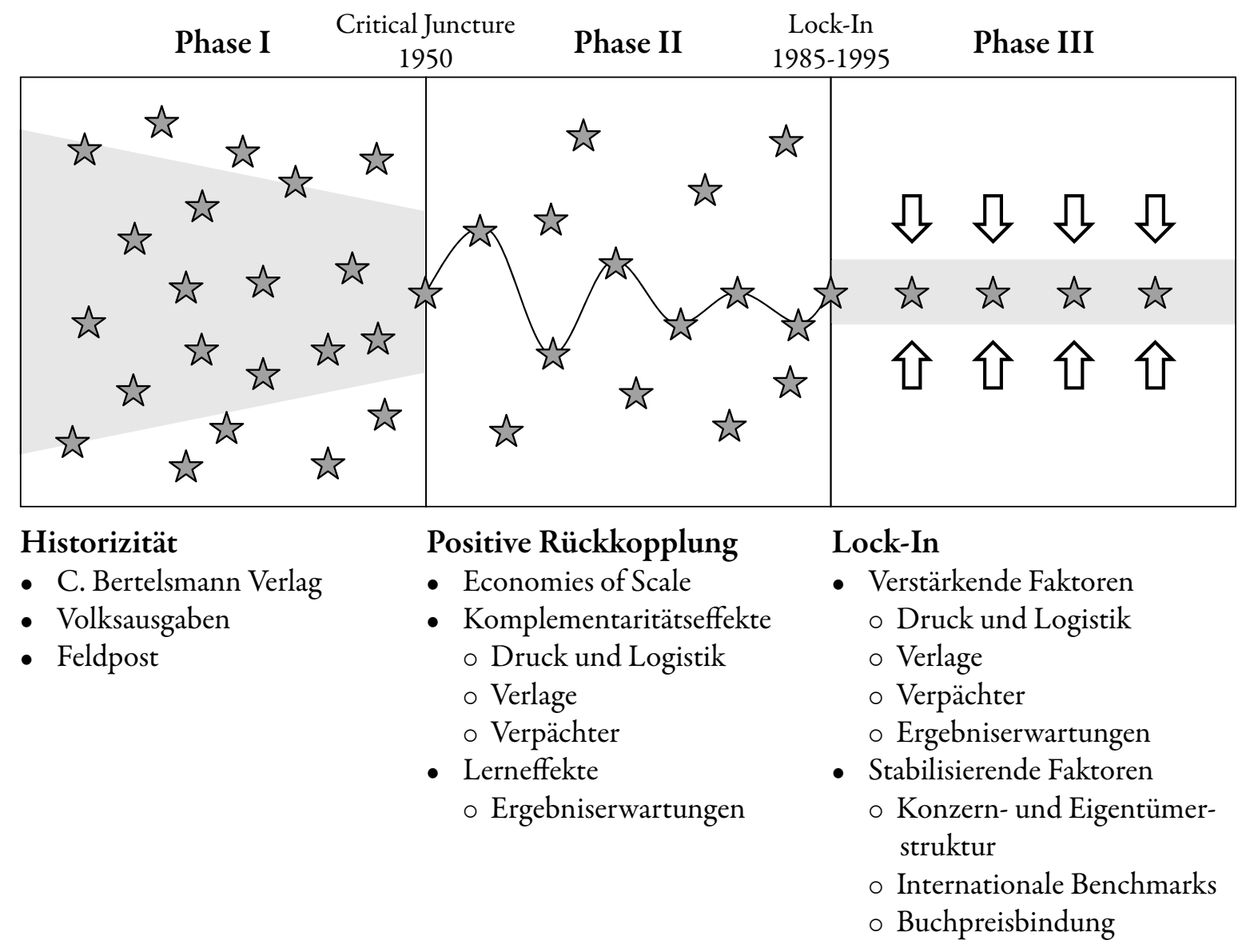

Quelle: Eigene Darstellung in Anlehnung an Schreyögg, G. et al. (2003), S. 272. 


\section{$5 \quad$ Fazit und Diskussion}

Abschließend werden die Ergebnisse der vorliegenden Arbeit zusammengefasst und kritisch gewürdigt. Dabei ist zu diskutieren, ob und inwiefern die Analyseergebnisse den an die Fallstudie gerichteten Gütekriterien der Konstruktvalidität, internen Validität sowie der Reliabilität gerecht werden. Schließlich wird die Verlässlichkeit der Ergebnisse aus der Perspektive alternativer Theorien zu organisationaler Trägheit untersucht.

\subsection{Zusammenfassung der Ergebnisse}

Die vorliegende Arbeit beschäftigte sich mit dem Entstehungsprozess von strategischer Pfadabhängigkeit am Beispiel des Bertelsmann Buchclubs in den Jahren von 1950 bis 2007.

Vor diesem Hintergrund erfolgte zunächst eine umfangreiche Diskussion zur Theorie der Pfadabhängigkeit in einem organisationalen Kontext. Diese galt es, von den zentralen Ansätzen zu organisationaler Trägheit und Rigidität (Imprinting, Structural Inertia, Lerntrajektorien und Kernkompetenzen sowie kognitive Verfestigungen) abzugrenzen. Es wurde herausgearbeitet, dass sich die Ansätze auf konzeptioneller Ebene nicht widersprechen, sondern sich im Gegenteil in das theoretische Gerüst der Pfadabhängigkeit integrieren lassen. Während die Analysedimensionen von Historizität und Kausalität in den meisten Ansätzen zu organisationaler Trägheit Berücksichtigung finden, liegt der Mehrwert der Pfadabhängigkeitstheorie in der zusätzlichen Betrachtung der Ebene des Mechanismus, mit welchem die einzelnen Entscheidungen innerhalb einer Prozesskette miteinander verknüpft sind. Die Theorie der Pfadabhängigkeit fokussiert sich somit nicht nur auf einzelne Entscheidungszeitpunkte, sondern zieht ihre analytische Erklärungskraft aus der Dynamik zwischen Entscheidungen im Entwicklungsverlauf. 
Zur Identifikation und Analyse von strategischer Pfadabhängigkeit auf Organisationsebene wurde auf Basis der zentralen pfadtheoretischen Annahmen ein zweischrittiges Modell zur Pfadanalyse entwickelt (Schritt 1: Rigiditätsnachweis; Schritt 2: Pfaddiagnose), dessen einzelne Komponenten die Messung von strategischer Rigidität und Pfadabhängigkeit ermöglichen.

Der empirische Teil wandte in einer holistischen Einzelfallstudie dieses Modell auf die strategische Entwicklung des deutschen Bertelsmann Buchclubs von 1950 bis 2007 an.

In Schritt 1 ließ sich ein kontinuierlicher Strategieprozess auf Ebene des Geschäftsmodells, der Corporate sowie der Business Strategy nachweisen. Um die Notwendigkeit zu Wandel und strategischer Anpassung darzulegen, wurde die seit den 80er Jahren einsetzende Dynamik des Buchmarkts als Systemumwelt anhand der Elemente Wettbewerber, Kunden und Substitutionsprodukte nachgezeichnet.

In Schritt 2 wurde anhand der Komponenten Historizität, Critical Juncture, positive Rückkopplung und Lock-In untersucht, ob die festgestellte Rigidität durch pfadabhängige Prozesse zu erklären ist.

Eine historische Vorprägung konnte sich noch vor der Clubgründung durch den Erfolg früher Geschäftsstrategien wie den Volks- und Feldpostausgaben, die der späteren Buchclubidee bereits ähnelten, feststellen lassen.

Als Critical Juncture und zugleich ,Big Event' ließ sich die Clubgründung als zweistufige Buchgemeinschaft benennen.

Zur Ermittlung der Komponente der positiven Rückkopplung wurde die strategische Entwicklung des Bertelsmann Buchclubs auf Selbstverstärkungsmechanismen hin untersucht. Aufgrund der Vielzahl theoretisch möglicher Mechanismen und selbstverstärkender Effekte zwischen unterschiedlichen Elementen innerhalb des Buchclubs einerseits sowie zwischen dem Buchclub und Umweltfaktoren andererseits wurde induktiv vorgegangen und das erhobene Datenmaterial zu Hinweisen auf Selbstverstär- 
kung überprüft. Es ließen sich mehrere Mechanismen der Selbstverstärkung ausmachen, die zu unterschiedlichen Zeitpunkten im Entwicklungsprozess der Pfadabhängigkeit anhand unterschiedlicher Effekte beobachtbar waren. Für den Entstehungsprozess der Pfadabhängigkeit konnten selbstverstärkende Effekte in Form von Skalenerträgen und Risikominimierung ausgemacht werden, die auf Basis von steigender Ertragskraft in der Wachstumsphase des Unternehmens das Entscheidungsverhalten bestätigten. Parallel dazu bildeten sich Komplementaritätseffekte zwischen dem Buchclub und den Kontextfaktoren Druckerei und Logistik, Eigenverlagen sowie den externen Vertriebsfirmen bzw. Verpächterorganisationen heraus. Diese steuerten wie schon die steigenden Skalenerträge zum Erfolg des Buchclubs bei, bewirkten jedoch bei nachlassendem Erfolg die Fortsetzung der strategischen Entscheidungstrajektorie trotz Änderungsbedarfs. Ähnlich wirkten Lerneffekte in Form von Ergebniserwartungen, die sich in der Wachstumsphase ausprägten und im weiteren Verlauf eine Einschränkung des strategischen Entscheidungsspielraums zur Folge hatten.

Als letzte Komponente der Pfaddiagnose ließ sich ein Lock-In ermitteln, das sich durch ökonomische Ineffizienz, Immunität gegenüber Wandlungsversuchen, unterschiedliche Formen von Ignoranz gegenüber Handlungsalternativen sowie stabilisierende Effekte auszeichnete.

Schließlich wurden die empirischen Befunde auf das Drei-Phasen-Modell der Pfadentstehung übertragen, um die einzelnen Stufen von der Pfadentstehung bis hin zur Pfadabhängigkeit im Fall des Bertelsmann Buchclubs in einen prozessualen Gesamtzusammenhang zu stellen.

Vor dem Hintergrund der empirischen Befunde ist abschließend über die Verlässlichkeit der Analyseergebnisse zu urteilen. Dies soll im folgenden Kapitel anhand der Gütekriterien der Konstruktvalidität, der internen Validität sowie der Reliabilität geschehen. 


\subsection{Zuverlässigkeit der Ergebnisse}

Im Rahmen einer kritischen Würdigung der Analyseergebnisse müssen vor allem zwei wesentliche Problemfelder offen angesprochen werden: der zu messende Forschungsgegenstand und das erhobene Datenmaterial.

Wie bereits intensiv im Theorieteil erläutert, lassen sich Pfadabhängigkeiten im Sinne von ARTHUR (1989) gemäß des stochastischen Modells der Polya-Urne metrisieren und den einzelnen Phasen vom Critical Juncture bis zum Lock-In zuordnen. Die Übertragung der Pfadtheorie auf strategische Entscheidungsprozesse entzieht sich dieser klaren analytischen Trennung, da strategische Entscheidungsverläufe in der Praxis hoch komplexe Prozesse darstellen. Wie erörtert, entstehen Entscheidungen sowohl intentional als auch emergent, können einander überlagern, auf unterschiedlichen Ebenen ablaufen und sind faktisch erst in Form von getroffenen Entschlüssen zu beobachten, obwohl sich die Phase der Entscheidungsfindung unter Umständen über einen langen Zeitraum erstreckt hat. Besonders bei der Erhebung von Selbstverstärkungsmechanismen z. B. in Form von Lerneffekten offenbart sich die Schwierigkeit, einen verlässlichen Kausalzusammenhang herzustellen, der auf den dahinter liegenden Mechanismus positiver Rückkopplungen schließen lässt.

Um das Phänomen strategischer Pfadabhängigkeit dennoch möglichst zuverlässig erheben zu können, wurde das zweischrittige Modell der Pfadanalyse entwickelt. Hierzu wurden zunächst die zu bestimmenden Konstrukte der strategischen Rigidität und Pfadabhängigkeit in einzelne Komponenten bzw. Elemente zerlegt, aus denen sich wiederum konkrete Indikatoren zur empirischen Erhebung ableiten ließen. Durch diese stufenweise Operationalisierung sollte eine möglichst breite Übereinstimmung der Erhebungsergebnisse mit den zu bestimmenden Konstrukten der Rigidität und Pfadabhängigkeit erreicht und ein Höchstmaß an Konstruktvalidität gewährleistet werden. 
Neben dem hohen Abstraktionsgrad des Untersuchungsgegenstandes ist die Zuverlässigkeit der Analyseergebnisse zusätzlich vor dem Hintergrund der Datenlage kritisch zu diskutieren. Allein der zeitliche Analyserahmen erstreckte sich über einen sehr langen Zeitraum von 57 Jahren. Hinzu kommt, dass der Bertelsmann Buchclub mittlerweile Teil eines multinationalen Medienkonzerns ist, dessen strategische und strukturelle Verflechtungen mit dem Buchclub für die Pfadanalyse gleichermaßen relevant waren. Das zu erhebende Datenmaterial musste somit sowohl zeitlich als auch inhaltlich einen sehr großen Umfang abdecken, um zuverlässige Schlüsse auf die strategische Entwicklung des Buchclubs ziehen zu können.

Im Gegensatz zu einer Vielzahl an verfügbaren Presseartikeln, Beiträgen in Fachzeitschriften und Imagebroschüren, welche die wesentlichen Meilensteine der Unternehmensgeschichte nachzeichnen, gestaltete sich die Recherche nach Dokumentationen über interne strategische Entscheidungsprozesse schwieriger. Eine große Hilfe bot vor allem das umfangreiche Unternehmensarchiv der Bertelsmann AG, das zahlreiche Akten und Dokumente zur Verfügung stellte. Während z. B. für die 60er und 70er Jahre eine sehr breite Datenbasis vorhanden und einsehbar war, gestaltete sich die Datensichtung aufgrund der einzuhaltenden Sperrfrist von 30 Jahren für vertrauliche Dokumente als problematisch, so dass sich vor allem die strategischen Entscheidungsprozesse ab den 80er Jahren nicht lückenlos durch das Archivmaterial erschließen ließen.

Selbst das einsehbare Archivmaterial ließ sich nicht unkritisch als repräsentativ für die tatsächlichen Entscheidungsprozesse übernehmen, da der vorhandene Aktenbestand des Bertelsmann Unternehmensarchivs zwar sehr umfangreich und abteilungsübergreifend vorliegt, jedoch auf freiwilliger Basis der einzelnen Abteilungen archiviert wurde. Vor diesem Hintergrund ist davon auszugehen, dass eine Vielzahl weiterer wichtiger Dokumente erst gar nicht in den Archivbestand aufgenommen wurde. 
Um die Reliabilität der Erhebungsergebnisse zu erhöhen, wurde daher auf eine möglichst breite Datenbasis aus unterschiedlichen Quellen geachtet (Triangulation). Vor allem durch Interviews mit direkt betroffenen ehemaligen und aktuellen Entscheidungsträgern des Buchclubs und der Bertelsmann AG ließen sich erste Ergebnisse der Dokumentenauswertung rückspiegeln und somit verifizieren bzw. verwerfen. Ein weiterer Beitrag zur Erhöhung der Reliabilität ließ sich durch die transparente Dokumentation des erhobenen Datenmaterials und der jeweiligen Untersuchungsergebnisse erreichen.

Schließlich sind die Resultate der Fallstudie auf interne Validität hin zu überprüfen, um zu klären, ob sich die beobachtete Rigidität im Fall des Bertelsmann Buchclubs nicht passender durch alternative Erklärungsansätze begreifen lässt. Um dem Anspruch von interner Validität möglichst gerecht zu werden, wurden bereits im Theorieteil der Arbeit weitere Ansätze zu organisationaler Trägheit mit der Pfadtheorie abgeglichen und Gemeinsamkeiten bzw. Unterschiede herausgearbeitet. Vor diesem Hintergrund wurde die strategische Entwicklung des Bertelsmann Buchclubs aus einer pfadtheoretischen Perspektive analysiert. Auf Basis der Analyseergebnisse sollen im nun folgenden Kapitel die strategischen Beharrungstendenzen und Wandlungswiderstände des Buchclubs aus der Perspektive alternativer Theorien diskutiert werden, um die Verlässlichkeit der pfadtheoretischen Erklärungskraft im vorliegenden Fall zu überprüfen. Hierzu bieten sich die bereits theoretisch behandelten Ansätze des Imprintings, Structural Inertia, Kernkompetenzen und Lerntrajektorien sowie kognitive Verfestigungen an.

\subsection{Weiterführende Diskussion}

Der Ansatz des Imprintings eignet sich insofern zur Erklärung der strategischen Kontinuität des Bertelsmann Buchclubs, als dass er zentrale Entscheidungen vor allem in der Gründungsphase eines Unternehmens als Ursache für einen kontinuierlichen Un- 
ternehmensverlauf heranzieht. Die Tatsache, dass die Gründung des Buchclubs im Jahr 1950 zugleich den Startpunkt des Analyserahmens bildet, lässt eine kontinuierliche Beobachtung der zum Zeitpunkt der Gründung terminierten Säulen des Geschäftsmodells zu. Die Analyseergebnisse zeigten, dass an den Ursprungsentscheidungen, eine mitgliedsgebundene zweistufige Buchgemeinschaft im Direktvertrieb zu gründen, kontinuierlich festgehalten wurde. Der Gründungseinfluss scheint im Fall des Bertelsmann Buchclubs folglich immanent. Unklar bleibt jedoch, aus welchen Gründen die ursprüngliche Strategie repliziert wurde und wie die einzelnen Entscheidungen im Zeitverlauf miteinander zusammenhängen. So lässt sich zwar eine Korrelation zwischen den frühen Gründungsentscheidungen und der späteren strategischen Ausrichtung feststellen, allerdings kann nicht erklärt werden, ob die strategischen Entscheidungen mehr als fünfzig Jahre nach der Gründung des Clubs in einem kausalen Zusammenhang mit dessen Konstitution stehen. Gegen eine Kausalität würde z. B. sprechen, dass die zentralen Gründungspersonen selbst nur noch bedingt Einfluss auf die operativen Geschäftsaktivitäten ausüben und die Geschäftsführung im Zeitverlauf sehr häufig wechselte.

In engem Zusammenhang mit der Pfadtheorie verweist auch die Theorie des Structural Inertia auf die Historiziät von Entscheidungen und die kontinuierliche Durchsetzung von Standards. So wird auf die Institutionalisierung von Normen und die Etablierung klarer Regeln in Unternehmen verwiesen, die ein Aufbrechen alter Strukturen erschweren und mit hohen Wechselkosten verbunden sind. Im Fall von Bertelsmann lassen sich die an den Club gerichteten rigiden Ergebniserwartungen als institutionelle Normen auffassen, die der Ausweitung des Programmangebots entgegenwirkten und die Refokussierung auf das Buch als Produkt und den ursprünglichen Clubgedanken unterstützten. In diesem Sinne kann die strategische Persistenz auch auf strukturelle Trägheit zurückgeführt werden. Allerdings bietet der Ansatz keine Erklärung, auf welche Art die rigiden Ergebniserwartungen und Zielvorgaben entstan- 
den sein könnten. Mit anderen Worten: Die Theorie des Structural Inertia ist hilfreich bei der Suche nach Gründen für die Wandlungswiderstände und Handlungsstarre des Clubs, besitzt allerdings nur begrenzte Aussagekraft zu der Art und Weise des Entstehungsprozesses.

Die theoretischen Ansätze zu Kernkompetenzen und zum ,Exploitation'-Lernen beziehen sich auf spezifische Lernprozesse, in denen ein Unternehmen auf der einen Seite bestimmte, vor allem zu Erfolg führende Fähigkeiten ausbildet, auf der anderen Seite jedoch andere Fähigkeiten oder Kompetenzen vernachlässigt und nicht aufbaut, so dass die Möglichkeit zum Wandel sukzessive verlernt wird. Im Fall des Bertelsmann Buchclubs lässt sich der Aufbau einer starken Vertriebskompetenz zum Massenabsatz nachzeichnen. Durch die Zweistufigkeit wurde bereits zum Gründungszeitpunkt das bestehende Netz des Reise- und Versandbuchhandels genutzt. Später folgten der Werbende Buch- und Zeitschriftenhandel und schließlich die stationären Sortimentsbuchhändler. Im weiteren Verlauf der Unternehmensgeschichte wurden auch eigene Werbemaßnahmen geschaltet und intensiv ausgebaut. Vertreterwerbung wurde durch Freundschafts-, schriftliche und Ladenwerbung ergänzt, mit dem Ziel, den Mitgliederbestand und die Buchverkaufszahlen zu steigern. Im Laufe der Zeit wurden umfassende Kundendatenbanken mit Informationen zu Soziodemographie, Kaufverhalten und -präferenzen entwickelt, um den sich erhöhenden Mitgliederbestand überdies gezielter ansprechen und die Kauffrequenz steigern zu können. Die klare Fokussierung auf die Kernkompetenz ,Vertrieb', die konsequente Zielverfolgung, den Mitgliederbestand zum Massenabsatz auszudehnen, lässt sich somit anhand der kontinuierlichen Ausweitung der Vertriebskompetenz ablesen.

Dass diese Kernkompetenz sich zunehmend zu einer Kernrigidität verfestigt hat, wird daran deutlich, dass die sich bereits in den 80er Jahren abzeichnende Notwendigkeit zum strategischen Wandel und zum Aufbau alternativer Geschäftsstrategien nicht wahrgenommen wurde. Unklar und durch den Ansatz nicht erklärbar bleibt je- 
doch, wie es zur Ausbildung der Rigidiät kam und durch welche Mechanismen die einzelnen Schritte der Trajektorie miteinander verknüpft sind. Nicht zu erklären ist ebenso wenig, dass der Buchclub trotz der eindeutigen Vertriebsausrichtung und der damit verbundenen Kompetenzausstattung (z. B. Kundenbindung in Mitgliedschaftssystemen) schließlich dennoch die Fähigkeit besaß, mit Boulevard, BOL und Best'Seller alternative Geschäftsstrategien zu entwickeln. Auch wenn es sich nicht um reine Diversifikationsstrategien, sondern um Markt-Erweiterungen handelte, bewegte sich der Club durch den Aufbau von eigenen Buchhandelsketten, dem Online-Handel sowie der mitgliedschaftsfreien Versandidee in bislang fremden Marktsegmenten. Der Ansatz der Kernrigidität greift somit in diesem Fall nur bedingt, da die prozessualen Verknüpfungen in der Ausbildung der Rigidität unberücksichtigt bleiben und strategische Abweichungen schwer erklärbar sind.

Schließlich beschreiben kognitive Verfestigungen Phänomene von selektiver Wahrnehmung und geteilter Denkschemata, die eine eingeschränkte, subjektive Sichtweise und das Ausblenden relevanter Markt- und Wettbewerbsveränderungen zur Folge haben können. Als Konsequenz steigt die Gefahr, notwendige strategische Anpassungen nicht zu erkennen. Im Fall des Bertelsmann Buchclubs ist es zwar prinzipiell möglich, die langjährige Missachtung der Marktsättigungstendenzen und Risikoprognosen durch Prozesse von selektiver Wahrnehmung zu erklären. Spätestens Mitte der 90er Jahre lässt sich die Argumentationslinie jedoch nicht mehr durchhalten, da die strukturellen Schwierigkeiten angesichts erodierender Mitgliederzahlen und Verlusten klar gesehen und benannt wurden. Dass dennoch keine strategischen Anpassungen erfolgten, ist schwer mit dem Ansatz der selektiven Wahrnehmung zu erfassen.

Mit ähnlichen Schwierigkeiten ist die Anwendung des Ansatzes der geteilten Denkmuster auf die Strategieentwicklung des Buchclubs verbunden. Selbst wenn sich kognitive Verfestigungen im Form von systemspezifischen Interpretationsschemata 
und Denkschablonen im Managementteam ausgebildet hatten, so dürften diese durch den mehr als zehnfachen Austausch der Geschäftsführung sowie der hohen Fluktuation im gesamten Managementteam aufgebrochen bzw. gelockert worden sein.

Es lässt sich festhalten, dass einzelne Phänomene der strategischen Entwicklung des Bertelsmann Buchclubs durch die Ansätze des Imprintings, Structural Inertia, Kernkompetenzen und Lerntrajektorien sowie kognitiven Verfestigungen erklärt werden können, diese jedoch zu kurz greifen, um die strategische Rigidität vollkommen erschließen zu können. Der partiellen Erklärungskraft der alternierenden Ansätze wird durch die Theorie der Pfadabhängigkeit insofern Rechnung getragen, als dass die Ansätze nicht im Gegensatz zu den pfadtheoretischen Annahmen stehen, sondern zentrale Argumente integriert und durch den Mechanismus der positiven Rückkopplung um eine Prozessperspektive erweitert werden.

Abschließend ist zu diskutieren, ob der Bertelsmann Buchclub „fast schon ein Auslaufmodell “"663 und die vorerst letzte Stufe des Produktlebenszyklus erreicht worden ist. Sicherlich hat sich gerade angesichts der aktuellen Wettbewerbssituation im Buchmarkt die Form eines mitgliedschaftsgebundenen Clubsystems dem Vorwurf auszusetzen, kein zeitgemäßes Geschäftsmodell mehr zu sein. Im Gegensatz dazu bekräftigt Bertelsmann immer wieder die Hoffnung auf zukünftiges Wachstum und die Möglichkeit zum Turnaround: „Denn dass Clubs überhaupt nicht funktionieren, gilt nicht mehr als Ausrede. Konkurrent Weltbild hat in Deutschland gezeigt, dass es besser geht, und auch bei Bertelsmann haben fast alle ausländischen Clubs, so jedenfalls der Chef der DirectGroup, Ewald Walgenbach, mittlerweile die Gewinnschwelle erreicht." ${ }_{664}$

Die Tatsache, dass das clubfreie Versandunternehmen Weltbild, Buchhandelsketten wie Hugendubel und Thalia sowie der Online-Versender Amazon.de jährliche 
Zuwachsraten verbuchen, beweist zumindest, dass es möglich ist, auch in einem hoch kompetitiven deutschen Buchmarkt erfolgreich agieren zu können. ${ }^{665}$

Inwiefern es dem Bertelsmann Buchclub gelingt, sich trotz der wirtschaftlichen Schwierigkeiten langfristig am Markt zu behaupten, wird sich in den nächsten Jahren zeigen. Ein erster wichtiger Schritt auf dem Weg zur nachhaltigen Erfolgssicherung ist sicherlich, zunächst die Fesseln der Pfadabhängigkeit zu erkennen und durch gezielte Maßnahmen den verstärkenden und stabilisierenden Faktoren entgegenzuwirken.

665 Vgl. Börsenblatt Online (2006, 28.12.); Börsenblatt Online (2007, 17.01.); Börsenblatt Online (2007, 20.08.). 



\section{Literaturverzeichnis}

ACKermanN, R. (2003): Die Pfadabhängigkeitstheorie als Erklärungsansatz unternehmerischer Entwicklungsprozesse, in: Schreyögg, G./Sydow, J. (Hrsg.): Strategische Prozesse und Pfade, Managementforschung 13, Wiesbaden, S. 225-255.

ACKERMANN, R. (2001): Pfadabhängigkeit, Institutionen und Regelreform, Tübingen.

Adron, L. (1983): Wissenswertes von A bis Z. 150 Jahre Bertelsmann. Die Bücher unserer Welt, Berlin.

AMAZON.DE (2008A): ,Amazon.com jetzt auch auf dem deutschen Buchmarkt vertreten', Pressemitteilung v. 15.10.1998, online, URL: http://www.amazon.de/ gp/press/pr/19981015/ref=amb_link_34364365_1?pf_rd_m=A3JWKAKR8XB7XF \&pf_rd_s=center-2\&pf_rd_r=18CEGHMDVNMGZBT5EET3\&pf_ rd_t=2701\&pf_rd_p=112870091\&pf_rd_i=home-1998, [10.02.2008].

AMAZON.DE (2008B): ,Amazon.de bietet persönliche Buch-Empfehlungen im Internet', Pressemitteilung v. 01.12.1999, online, URL:

http://www.amazon.de/gp/press/pr/19991201/ref=amb_link_34364265_1?pf_rd_ $\mathrm{m}=$ =A3JWKAKR8XB7XF\&pf_rd_s=center2\&pf_rd_r=1S3S5MEKNYGGEXYNC SXN\&pf_rd_t=2701\&pf_rd_p=112899591 \&pf_rd_i=home-1999, [10.02.2008].

AMAZON.DE (2008C): ,Über uns', online, URL: http://www.amazon.de/gp/press/info/ home, [10.02.2008].

AMAZON.DE (2008D): ,Amazon.de eröffnet Marketplace', online, URL:

http://www.amazon.de/gp/press/pr/20020320/ref=amb_link_34363965_34?pf_rd_ $\mathrm{m}=$ A3JWKAKR8XB7XF\&pf_rd_s=center2\&pf_rd_r=02C9VT1N15TEZYS72W7 T\&pf_rd_t=2701\&pf_rd_p=112899391\&pf_rd_i=home-2002, [10.02.2008].

AMAZON.DE (2008E): ,Zeitschriften und Magazine bei Amazon.de', online, URL: http://www.amazon.de/gp/press/pr/20021107/ref=amb_link_34363965_7?pf_rd_ $\mathrm{m}=$ A3JWKAKR8XB7XF\&pf_rd_s=center2\&pf_rd_r=02C9VT1N15TEZYS72W 7T\& pf_rd_t=2701\&pf_rd_p=112899391\&pf_rd_i =home-2002, [10.02.2008].

AMAZON.DE (2008F): ,Amazon.de startet DVD-Verleih in Deutschland', online, URL: http://www.amazon.de/gp/press/pr/20050623/ref=amb_link_34363565_20?pf_rd_ $\mathrm{m}=$ =A3JWKAKR8XB7XF\&pf_rd_s=center-2\&pf_rd_r=0ZT4H15PQFSHTETCR XRC\& 2 p_rd_t=2701\&pf_rd_p=112865191\&pf_rd_i=home-2005, [10.02.2008]. 
AMAZON.DE (2008G): , Amazon.de bringt Kreditkarte heraus: Bei jedem Einkauf Amazon.de Punkte sammeln - online und offline', Pressemitteilung v. 03.11.2006, online, URL: http://www.amazon.de/gp/press/pr/20061103/ref=amb_link_3436346 5_9? pf_rd_m=A3JWKAKR8XB7XF\&pf_rd_s=center-2\&pf_rd_r=19B4A2CMX27 B6MDGRD07\&pf_rd_t=2701\&pf_rd_p=132742291\&pf_rd_i $=$ home-2006, [10.02.2008].

ANSOFF, H.I. (1968): Corporate Strategy, Bungay.

ARrow, K.J. (2003): Path dependence and competitive equilibrium, in: Guinnane, T. et al. (Hrsg): History matters. Essays on economic growth, technology, and demographic change, Chicago, S. 23-35.

ARTHUR, W.B. (1989): Competing technologies, increasing returns, and lock-in by historical events, in: Economic Journal, Vol. 99, S. 116-131.

ARVATO.COM (2008): ,Bereiche', online, URL: http://www.arvato.com/index.php? LANG=de\&PAGE=bereiche, [04.01.2008].

ASHBY, W.R. (1965): An introduction to cybernetics, London.

BECKER, A. (1996): Rationalität strategischer Entscheidungsprozesse: Ein strukturationstheoretisches Konzept, Wiesbaden.

BEINSEN-RUf, H.-L. (1980): Kalkulation der Buchgemeinschaft, dargstellt an den Clubs des Bertelsmann-Konzerns. Diplomarbeit an der Fachhochschule für Druck Stuttgart im Studiengang Verlagswirtschaft und Verlagsherstellung im WS 1979/80.

BERTELSMANN AG (1985): 1835-1985. 150 Jahre Bertelsmann, München.

BERTELSMANN AG (1985A): Wirtschaftliche Eckdaten des Lesering-Geschäfts, Diskussionsgrundlage für die außerordentliche Beiratssitzung, 30.09.1985.

BERTELSMANN.DE (2008A): ,Bertelsmann-Chronik', online, URL: http://www.bertelsmann.de/bertelsmann_corp/wms41//customers/bmcorp/pdf/Be rtelsmann-Chronik_1835-2007_.pdf, [05.01.2008].

BERTELSMANN.DE (2008B): ,Gruner+Jahr: Qualitätsjournalismus Seite für Seite', online,URL:http://www.bertelsmann.de/bertelsmann_corp/wms41/bm/index.php ?ci=25\&language $=1$, [05.01.2008].

BERTELSMANN.DE (2008C): ,RTL Group: Gute Unterhaltung auf allen Kanälen', online,URL:http://www.bertelsmann.de/bertelsmann_corp/wms41/bm/index.php? $\mathrm{ci}=168$ \&language $=1$ \&pagesize $=\mathrm{A} \&$ pagecolor $=$ normal, [07.01.2008] .

BERTELSMANN.DE (2008D): ,Ein Unternehmen - sechs Bereiche', online, URL: http://www.bertelsmann.de/bertelsmann_corp/wms41/bm/index.php?ci=25\&lang uage $=1,[10.01 .2008]$. 
BERTELSMANN.DE (2008E): ,Die Hühnerfarm Hennengold', online, URL: http://www.bertelsmann.de/bertelsmann_corp/wms41//customers/bmcorp/pdf/Di e_H_hnerfarm_Hennengold.pdf, [17.01.2008].

BEYER, J. (2006): Pfadabhängigkeit. Über institutionelle Kontinuität, anfällige Stabilität und fundamentalen Wandel, Frankfurt/M.

BEYER, J. (2005): Pfadabhängigkeit ist nicht gleich Pfadabhängigkeit! Wider den impliziten Konservatismus eines gängigen Konzepts, in: Zeitschrift für Soziologie, Jg. 24, Heft 1, S. 5-21.

BG (2006): Geschäftsbericht 2006, Bertelsmann AG (Hrsg.).

BG (2004): Geschäftsbericht 2004, Bertelsmann AG (Hrsg.).

BG (2003): Geschäftsbericht 2003, Bertelsmann AG (Hrsg.).

BG (2002): Geschäftsbereicht 2002, Bertelsmann AG (Hrsg.).

BG (2000/01): Geschäftsbericht 2000/01, Bertelsmann AG (Hrsg.).

BG (1999/2000): Geschäftsbericht 1999/2000, Bertelsmann AG (Hrsg.).

BG (1998/99): Geschäftsbericht 1998/99, Bertelsmann AG (Hrsg.).

BG (1997/98): Geschäftsbericht 1997/98, Bertelsmann AG (Hrsg.).

BG (1994/95): Geschäftsbereicht 1994/95, Bertelsmann AG (Hrsg.).

BG (1993/94): Geschäftsbericht 1993/94, Bertelsmann AG (Hrsg.).

BG (1992/93): Geschäftsbericht 1992/93, Bertelsmann AG (Hrsg.).

BG (1991/92): Geschäftsbericht 1991/92, Bertelsmann AG (Hrsg.).

BG (1988/89): Geschäftsbericht 1988/89, Bertelsmann AG (Hrsg.).

BG (1987/88): Geschäftsbericht 1987/88, Bertelsmann AG (Hrsg.).

BG (1985/86): Geschäftsbericht 1985/86, Bertelsmann AG (Hrsg.).

BG (1984/85): Geschäftsbericht 1984/85, Bertelsmann AG (Hrsg.).

BG (1977/78): Geschäftsbereicht 1977/78, Bertelsmann AG (Hrsg.).

BG (1973/74): Geschäftsbericht 1973/74, Bertelsmann AG (Hrsg.).

BI (1970/01): ,Technische Betriebe', BUA-UP2/1, Multhaupt, H. (Verf.), [25.01.2006].

BI (1969/04): ,Der Bertelsmann Sachbuchverlag', BUA-UP2/1, Schmoeckel, P. (Verf.), [23.01.2006].

BI (1969/03): ,Das Verlagsportrait: Der Bertelsmann Jugendbuchverlag', BUAUP2/1, Scholz, J. (Verf.), [23.01.2006]. 
BI (1969/02): ,Das Verlagsportrait: Der Bertelsmann Lexikon-Verlag', BUA-UP2/1, Lenz, W. (Verf.), [25.01.2006].

BI (1969/01): ,Das erste Jahr Verlagsgruppe', BUA-UP2/1, Wendorff, R. (Verf.), [24.01.2006].

BI (1968/01): ,Verlagsgruppe Bertelsmann', BUA-UP2/1, Wendorff, R. (Verf.), [23.01.2006].

BI (1966/03): ,Besuch aus Wien', BUA-UP2/1, o. V., S. 2f., [23.01.2006].

BI (1966/01-02): ,Bericht über das Geschäftsjahr 1965', BUA-UP2/1, Mohn, R. (Verf.), [23.01.2006].

BI (1964/01-02): ,1964: Die vierte Phase unserer Entwicklung beginnt', Mohn, R. (Verf.), [23.01.2006].

BI (1963/03-04): ,Betriebsrat hörte Bericht über Lage und Entwicklung der Bertelsmann-Firmen', BUA-UP2/1, o. V., [25.01.2006].

BI (1962/08-09): ,Wie geht es weiter mit der Ariola', BUA-UP2/1, Interview mit Rudolf Engleder, [23.01.2006].

BI (1962/01): ,Bertelsmann GmbH', BUA-UP2/1, Wixforth, F. (Verf.), S. 3, [24.01.2006].

BI (1961/09): ,Europaring stellt sich vor', BUA-UP2/1, o. V., S. 7, [25.01.2006].

BI (1960/09): ,Zeittafel', Sonderausgabe 125 Jahre Bertelsmann, BUA-UP2/1, [23.01.2006].

BI (1960/01): ,Bertelsmann GmbH', BUA-UP2/1, o. V., [24.01.2006].

BI (1959/07): ,Geschäftsbericht II. Quartal 1958', BUA-UP2/1, Köhnlechner (Verf.), [23.01.2006].

BI (1959/04): ,Geschäftsbericht I. Quartal 1959', BUA-UP2/1, Mohn, R. (Verf.), [23.01.2006].

BI (1956-57/JAHRESWENDE): ,Jahresbericht 1956', BUA-UP2/1, Mohn, R. (Verf.), [24.01.2006].

BI (1956/05): ,Heim und Buch', BUA-UP2/1, o. V., [25.01.2006].

Blana, H. (1993): Die Herstellung, in: Göhler, W./Merzbach, J. (Hrsg.):

Grundwissen Buchhandel - Verlage, Band 5, 3. Aufl., München u. a.

BLUMBERG, B. ET AL. (2005): Business research methods, Maidenhead.

BöCKelmanN, F./FIsCHLER, H. (2004): Bertelsmann. Hinter der Fassade des Medienimperiums, Frankfurt/M. 
BOEKER, W. (1989): Strategic change: The effects of founding and history, in: The Academy of Management Journal, Vol. 32, Nr. 3, S. 489-515.

BÖRSENBLATT (2003/38): ,Wie aus einem Guss', Interview mit Geschäftsführer Rainer Wittenberg, Nr. 38, S. 18-21.

BÖRSENBLATT (2002, 26.07.): ,Romanik auch für Club-Mitglieder', Nr. 59, S. 5.

BÖRSENBLATT (2001, 30.11.): ,Der Club Bertelsmann “Wir mussten einfach reagieren"', Interview mit Geschäftsführer Wulf Böttger, Nr. 96, S. 8-11.

BöRSENBLATT (1999, 16.11.): ,Bertelsmann: Clubs stehen wieder besser da', Nr. 91, S. 5.

BÖRSENBLATT (1996, 12.11.): ,Neue Welt der Bücher', Nr. 91, S. 5.

BÖRSENBLATT (1983, 23.12.): ,Bertelsmann: Wössner gewählt', Nr. 107, S. 2763.

BÖRSENBLATT $(1983,31.03):$. Das Buch bleibt Schwerpunkt Nummer eins', Interview mit Lesering-Chef Dr. Hans Zopp, Nr. 26, S. 817.

BÖrSENBLATT (1983, 08.03.): ,Clubs expandieren mit Videos', Nr. 19, S. 539.

BÖrSENBLATt (1977, 27.05.): ,Von Eduscho lernen ...', Jaspert, F. (Verf.), Nr. 42, S. 79-82.

BöRSEnblatT (1976, 25.06.): ,Todsicheres Rezept', Opitz, A. (Verf.), Nr. 51, S. $951 f$.

BÖRSENBLATT $(1975,12.09):$. ,Spricht Bertelsmann bald mit dem Schah?', Nr. 73, S. 1198.

BÖRSENBLATT (1974, 17.05.): ,Lesekultur in Deutschland 1974', Schmidtchen, G. (Verf.), Nr. 39, S. 707-770.

BÖrSENBLATT (1973, 20.02.): ,Wachsende Märkte für das Buch?', Lucius, W. D. v. (Verf.), Nr. 14, S. 204-211.

BÖRSENBLATT (1972, 03.11.): ,Andere Leser - andere Bücher?’, Nr. 88, S. 2458.

BÖRSENBLATT $(1970,12.05$.$) , ,Der befragte Leser', Muth, L. (Verf.), Nr. 38,$ S. 1015-1034.

BörSEnblatT (1969, 20.08.): ,Viele Hunde sind des Hasen Tod', Ulmer, R. (Verf.), Nr. 69, S. 1844-1854.

BÖrSENBLATT (1966, 07.01.): ,Was bedeutet Demoskopie für den Buchhandel?', Muth, L. (Verf.), Nr. 2, S. 20-24.

BÖRSENBLATT $(1963,26.04$.$) : ,Das Schallplattengeschäft 1962', Nr. 34, S. 660 f$.

BÖRSENBLATT $(1959,17.03$.$) , ,Die Umsatzentwicklung des Sortimentsbuchhandels$ im Jahre 1958', Abt. Marktanalyse (Verf.), Nr. 22, S. 278. 
BÖRSENBLATT (1954, 04.05.): ,Vorausschau auf das Jahr 1954', Ruf, K. (Verf.), Nr. 35, S. 255-257.

BörSEnblatt Online (2007, 08.08.): , Thalia legt um 25,9 Prozent zu'.

BÖRSENBLATT ONLINE (2007, 17.01.): ,Douglas-Holding setzt Wachstumskurs fort'.

BÖRSENBLATT ONLINE (2007, 28.12.): ,Amazon mit bestem Weihnachtsgeschäft aller Zeiten'.

BÖRSENBLATT ONLINE (2006, 23.08.): ,Sorgenkind Buchclub’.

BÖRSENVEREIN DES DEUTSCHEN BUCHHANDELS E.V. (HRSG.) (1950-2007): Buch und Buchhandel in Zahlen, Frankfurt/M., jährliche Erscheinungsweise, 1950-2007.

BörSENVEREIN (2006): Buchhandlungen und Neue Medien, Studienreihe Marktforschung, Börsenverein des Deutschen Buchhandels e.V. (Hrsg.), Frankfurt/M.

BÖRSENVEREIN (1963): Buch und Buchhandel in Zahlen, Börsenverein des Deutschen Buchhandels e.V. (Hrsg.), Frankfurt/M., Ausgabe 1963.

BOERSENVEREIN.DE (2008A): ,Titelproduktion 2006', online, URL: http://www.buchnrw.de/404_index.php, [14.01.2008].

BOERSENVEREIN.DE (2007A): ,Der Börsenverein des Deutschen Buchhandels e.V.', online, URL: http://www.boersenverein.de/de/64629, [09.12.2007].

BOERSENVEREIN.DE (2007B): ,Branchen-Monitor BUCH', 3. Quartal 2007, online, URL: http://www.boersenverein.de/de/169469, [09.12.2007].

BÖRSEN-ZEITUNG (1992, 27.02.): ,Bertelsmann übernimmt Deutschen Bücherbund', Nr. 40.

BÖRSEN-ZEITUNG (1990, 11.12.): ,Bertelsmann auf der Erfolgsschiene’, Nr. 238.

BÖRSEN-ZEITUNG (1989, 02.12.): ,Bertelsmann-Verlage expandieren', Nr. 233.

BÖTTGER, W. (2002, 11.09.): ,Kundenorientierung im Medienclub', Vortrag gehalten CRM-World-Kongress 2002.

BOGNER, A./MENZ, W. (2005): Das theoriegenerierende Experteninterview. Erkenntnisinteresse, Wissensformen, Interaktion, in: Bogner, A. et al. (Hrsg.): Das Experteninterview. Theorie, Methode, Anwendung, Opladen, S. 33-70.

BRACKER, J. (1980): The historical development of the strategic management concept, in: Academy of Management Review, Vol. 5, No. 2, pp. 219-224.

BRAMANN, K.-W. ET AL. (1993): Sortiments- und Verlagskunde. Grundwissen Buchhandel-Verlage, Band 2, München u. a. 
BRAMANN.DE (2007): ,Gesetz über die Preisbindung für Bücher', online, URL: http://www.bramann.de/down/Gesetz_Preisbindung.pdf, [12.12.2007].

BRONNER, R. (1992): Komplexität, in: Frese, E. (Hrsg.): Handwörterbuch der Organisation, 3. Aufl., Stuttgart, Sp. 1121-1130.

BUA (2006): Vorwort Findbücher Bestände des Leserings, [24.08.2006].

BUA-0006/15 (BERTELSMANN CLUBARBEIT: CHRONIK, 1977): ,Chronik des Aufbaus der Bertelsmann Clubarbeit dargestellt am Beispiel des Leserings A mit Kurzverweisen auf die anderen Clubs für die Jahre 1950-1977’, o. D., Oeltze, O. (Verf.), [23.01.2006].

BUA-0006/30 (LESERING: VerTRAG, 1970): Dokument v. 25.06.1970, o. V., [24.08.2006].

BUA-0006/73 (MARKTENTWICKLUNG, 1968): ,Entwicklung der Vertreterwerbung für Clubmitgliedschaften', Dokument v. 29.07.1968, Türnau (Verf.), [22.08.2006].

BUA-0006/75(2) (BEIRATSPROTOKOLL, 1968, 04.11.): Dokument v. 22.11.1968, Ringkamp/Oeltze, O. (Verf.), [26.07.2006].

BUA-0006/91 (VerlagSARBEIT, 1968): ,Zur Arbeit der Verlage', Dokument v. 01.07.1968, Wendorff, R. (Verf.), [24.08.2006].

BUA-0006/115 (MONDPROJEKT, 1969): ,Buch über Mondprojekt', Dokument v. 23.01.1969, Wendorff (Verf.), [22.08.2006].

BUA-0006/129 (ARBEIT DER BUCHGEMEINSCHAFTEN, 1970): Dokument v. 16.04.1970, Zopp, H. (Verf.), [27.07.2006].

BUA-0006/131 (ZehnjahresPlanUNG, 1970): Brief an Herrn Dr. Türnau, o. D., o. V., [24.08.2006].

BUA-0006/218(2) (LESERING: MitgliEDERUNTERSUCHUNG, 1971): ,Vergleichende Mitgliederuntersuchung LR/A und EBG', Dokument v. 07.10.1971, Abteilung BZK (Verf.), [24.08.2006].

BUA-0006/218(2) (RINGgESChäFT: AKTUELle Probleme, 1969): ,Aktuelle Probleme unseres Ringgeschäfts', Aufzeichnungen zu einem Vortrag vor Mitarbeitern, Dokument v. 13.05.1969, Oeltze, O. (Verf.), [27.07.2006].

BUA-0006/219(2) (VOLKSLEXIKON, 1971): Dokument v. 26.11.1971, Oeltze, O. (Verf.), [28.08.2006].

BUA-0006/272 (Programmbeirat, 1977): Dokument v. 12.10.1977, Bauer, J. (Verf.), [27.07.2006].

BUA-0006/455 (SONDEROBJEKTE, O.D.): ,Erweiterung des Angebots von Sonderobjekten in den Club-Illustrierten', o. D., o. V., [31.08.2006]. 
BUA-0006/464 (ZAHLENMATERIAL, 1974): ,Zahlenmaterial für Gespräche mit Fernsehleuten', Dokument v. 07.01.1974, o. V., [24.08.2006].

BUA-0006/464 (DEUTSCHE BUCHGEMEINSCHAFT, 1970): Dokument v. 20.04.1970, Mohn, R. (Verf.), [31.08.2006].

BUA-0006/464 (RING-WERBUNG, 1968): Dokument v. 26.08.1968, Hennig (Verf.), [31.08.2006].

BUA-0006/465 (HENNIG, 1977): o. D., o. V., [24.08.2006].

BUA-0039/1 (LESERING: GRÜNDUNG, 1950): Dokument v. 31.05.1950, Hornberg (Verf.), [24.08.2006].

BUA-0041/12 (LESERING: ANHANG ZUR BEITRITTSERKLÄRUNG, 1956): Dokument v. 01.06.1956, o. V., [25.08.2006].

BUA-0041/12 (LESERING: ANLEITUNG FÜR DIE VERTRIEBSFIRMEN, 1958): Dokument v. 1958, Bertelsmann GmbH (Verf.), [24.08.2006].

BUA-0041/6 (LESERING: UMFRAGE, 1956): ,Leseringmitglieder. Ergebnisse einer Umfrage, DIVO Markt- und Meinungsforschung GmbH, Frankfurt/M. (Hrsg.), [28.07.2006].

BUA-0041/2 (LESERING: ENTWICKLUNG UND AUfGABE, 1961): Dokument v. Juni 1961, Oelze, O. (Verf.), [27.07.2006].

BUA-0204/5 (BETRIEBSFORM DER BUCHGEMEINSCHAFT, O. D.): o. V., [24.08.2006].

BUA-0041/82(1) (BUCHGEMEINSCHAFTEN: FÜR UND WIDER, O. D.): o. V. [27.08.2006].

BUA-0041/82(1) (BUCHGEMEINSCHAFTEN: HISTORIE, 1959): Dokument v. 19.11.1959, o. V., [24.08.2006].

BUA-1000/27 (MITGLIEDERENTWICKLUNG, 1982): ,Mitgliederentwicklung bei deutschen Clubs', Dokument v. 14.10.1982, o. V., [27.07.2006].

BUA-UP2/128-1955 (LESERING-RUNDBRIEF, 1955): ,Bertelsmann Jugend-Lesering', o. D., Oeltze, O./Hennig, M. (Verf.), [25.07.2006].

BUA-UP2/129-169 (RUNDSCHREIBEN, 1969): ,Stand der Werbung für die Bertelsmann Ringe, Dokument v. 17.02.1969, Oeltze, O. (Verf.), [22.08.2006].

BUA-UP3/44-7 (LESEFAMILIE, 1988): Die größte Lesefamilie der Welt. Das Unternehmen Bertelsmann und seine Buchclubs, Dokument v. 1988, Bertelsmann AG (Hrsg.), [23.01.2006].

BUA-UP3/26-7 (BERTELSMANN ProsPeKT, 1959): Dokument v. 1959, o. V., [25.01.2006]. 
BUCHMARKT (2004/04): ,Club Bertelsmann testet im Internet und per Katalog ,Best!Seller' über 500 MA-Artikel', Dokument v. 16.04.2004, Online-Archiv, URL: www.buchmarkt.de, [05.12.2007].

BUCHMARKT (2003/02): ,Jetzt offizielle Pressemeldung zum „Boulevard“-Verkauf in Bielefeld', Dokument v. 05.02.2003, online-Archiv, URL: www.buchmarkt.de, [05.12.2007].

BUCHMARKT (2002/02): ,Boulevard-Buchhandlung Bielefeld startet am 28. Februar', Dokument v. 13.02.2002, Online-Archiv, URL: www.buchmarkt.de, [05.12.2007].

BUCHMARKT (2001/10): ,Bertelsmann Club geht in Marketing Offensive / Neue Buchkette geplant / Partner Jens Grüttefien', Dokument v. 04.10.2001, onlineArchiv, URL: www.buchmarkt.de, [05.12.2007].

BUCHMARKT (1999/09): , Weltbilds neuer Katalog „Jokers“", Dokument v. 02.09.1999, online-Archiv, URL: www.buchmarkt.de, [05.12.2007].

BuCHMARKT (1978/11): ,Weg vom Seifenplatz, das neue Gesicht der Club-Center', S. 42 .

BUCHREPORT (2006, 17.04.): ,Mit der Maus ins Kino', Nr. 17.

BUCHREPORT (2005, 13.10.): ,Clubs müssen Kasse machen', Nr. 41.

BUCHREPORT (2004, 08.07.): ,Bertelsmann Club und Weltbild im Vergleich', Nr. 28, S. 7.

BUCHREPORT (2003, 25.09.): ,Club-Pannen in den letzten 12 Jahren', Nr. 39.

BUCHREPORT (2002, 18.12.): ,Alle Jahre wieder', Nr. 51/52, S. 8.

BÜCHER, K. (1910): Das Gesetz der Massenproduktion, in: Zeitschrift für die gesamte Staatswissenschaft, S. 429-444; abgedruckt in Bücher (1920): Die Entstehung der Volkswirtschaft II, S. 89-106.

BurTON, M.D./BECKMAN, C.M. (2005): Leaving a legacy: Role imprints and successor turnover in young firms, paper presented at the ' $21^{\text {st }}$ EGOS colloquium' 2005, Berlin.

CHANDLER, A.D. (1962): Strategy and structure. Chapters in the history of industrial enterprise, Cambridge, MA.

Clubkatalog (2008/JANuar, Februar, MärZ): RM Buch und Medien Vertrieb $\mathrm{GmbH}$ (Hrsg.).

DANNEELS, E. (2002): The dynamics of product innovation and firm competences, in: Strategic Management Journal, Vol. 23, S. 1095-1121. 
DAVID, P.A. (1985): Clio and the Economics of QWERTY, in: American Economic Review, Vol. 75, S. 332-337.

DEARBORN, D.C./SimON, H.A. (1958): Selective perception: A note on the departmental identifications of executives, in: Sociometry, Vol. 21, Nr. 2, S. 140-144.

Der Club BerTelsmann (2008): Mitgliedschaftsdaten zum Bertelsmann Buchclub der Jahre 1995-2003, Abteilung Presse- und Öffentlichkeitsarbeit (Verf.), Daten elektronisch empfangen [28.04.2008].

DERCLUB.DE (2008A): ,Historie', online, URL: http://www.derclub.de/isbin/INTERSHOP.enfinity/eCS/Store/de/-/EUR/DEPresse-Chronik; sid=1ZFWNU6Mt5xWrgkFA72CCwCB_un_CiMvHfE=, [13.01.2008].

DeRCLUB.DE (2008B): 'Clubvereinbarungen', online, URL: http://www.derclub.de/, „Hilfe', [13.01.2008].

DERCLUb.DE (2008C): ,Viele Wege führen zum Club', online, URL: http://www.derclub.de/is-bin/INTERSHOP.enfinity/eCS/Store/de//EUR/DEPresseEinkaufsmoeglichkeiten;sid=n69jr5j6wGJjo99 wps23kdb3g9zHX ZZwP-Y=, [13.01.2008].

DeRCLUB.DE (2008D): ,Das Versprechen an unsere Kunden', online, URL: http://www.derclub.de/is-bin/INTERSHOP.enfinity/eCS/Store/de//EUR/DEPresseVersprechen;sid=LuBdCmvRfPNdHCxbF3yJNCXcF2HwjgZy5o $\mathrm{A}=$, [14.01.2008].

DERCLUB.DE (2008E): ,Home', online, URL: http://www.derclub.de/isbin/INTERSHOP.enfinity/eCS/Store/de/-/EUR/DEStorefrontStart;sid=IICZbxBxlKqZ2Vf_nUpNUV58iPM9nR77NMk=, [16.01.2008].

Der SPIEGEL (1998, 26.10.): ,Marketingmaschine Middelhoff, Nr. 44.

Deutsche NationalbibliotheK (2006): ,Börsenverein des Deutschen Buchhandels, Archiv und Bibliothek', Merkblatt.

DiEDRICH, H. (1992): Allgemeine Betriebswirtschaftslehre, 7. Auflage, Stuttgart u.a.

DiEVERNICH, F.E.P. (2007): Pfadabhängigkeit im Management, Stuttgart.

DIE WeLT (2006, 07.10.): 'Sorgenkinder gibt es nicht mehr', S. 14.

DiE WeLT (1998, 20.11.): ,Buchclub bremst Bertelsmann'.

DiE Welt (1989, 20.05.): ,Bertelsmann schließt die Buchclubs in Südamerika', Nr. 115.

DIE WeLt (1988, 29.02.): ,Bertelsmann lässt sich weltweit in die Bücher gucken’, Nr. 50.

DIE Welt (1986, 26.06.): ,Wahrung der Kontinuität bleibt die Maxime', Nr. 145.

DIE WelT (1983, 22.11.): ,Rückzug vom Gemischwarenhandel', Nr. 272. 
DiE Welt (1981, 01.11.): ,Mit dem Flexcover aus der Flaute', Nr. 254.

DIE ZeIT (1981, 22.05.): ,Vorrang für Gewinne’, Nr. 22.

DireCTGROUP-BERTELSMANN.DE (2008): ,Zahlen und Fakten zur DirectGroup', online, URL: http://www.directgroup-bertelsmann.de/cd/directgroup/ facts_figures.js,p [17.01.2008].

DöRRICH, S. (1991): Die Zukunft des Mediums Buch. Eine Strukturanalyse des verbreitenden Buchhandels, Bochumer Studien zur Publizistik- und Kommunikationswissenschaft, Band 67, Bochum.

DOUGHERTY, D. (1995): Managing your core incompetencies for corporate venturing, in: Entrepreneurship Theory and Practice, Vol. 19, Nr. 3, S. 113-135.

DudEN (1989): Deutsches Universalwörterbuch, 2. Aufl., Mannheim u.a.

EMERSON, R.M. ET AL. (1995): Writing ethnographic fieldnotes, Chicago.

FAS (2006, 01.10.): ,Hurra, wir lesen noch!', Nr. 39, S. 44.

FAuLSTICH, W. (1979): Buch, in: Faulstich, W. (Hrsg.): Kritische Stichwörter zur Medienwissenschaft, München, S. 48-84.

FAZ (2006, 23.08.): ,Mannschaftsleistung', Nr. 195, S. 16.

FAZ (2006, 27.06.): ,Reinhard Mohn 85 Jahre', Nr. 146, S. 16.

FAZ (2002, 19.10.): ,Bertelsmann stellt das Musikgeschäft auf den Prüfstand', Nr. 243, S. 14

FAZ (1992, 29.02.): ,Kirch gibt Bücherbund an Bertelsmann ab', Nr. 51.

FAZ (1989, 21.07.): ,Bertelsmann kooperiert mit VISA', Nr. 166.

FAZ (1986, 17.09.): ,Jetzt ist Bertelsmann der Welt größter Medienkonzern', Nr. 224.

FAZ (1986, 10.09.): ,Bertelsmann rückt auf Platz drei des internationalen Musikmarkts', Nr. 209.

FAZ (1985, 15.06.): ,Vielfalt als geistige Leitwährung', Nr. 136.

FAZ (1983, 22.11.): , Bertelsmann stellt sich auf eine Marktberuhigung ein’, Nr. 271.

FAZ (1983, 04.03.): ,Videoverleih auch bei Bertelsmann', Nr. 53.

FR (1989, 28.06.): , Buch ist Buch ist Buch bei Bertelsmann', Nr. 146.

Franzen, H. (1987): Die Preisbindung des Buchhandels, 3. Aufl., München.

FTD (2006, 26.09.): ,Bertelsmann sucht Rezepte für Buchclubs', S. 6.

FTD (2006, 06.06.): , Bertelsmann ringt um Buchklub-Profite', S. 6.

FTD (2002, 22.10.): ,Bertelsmann schließt BOL Deutschland', Nr. 204, S. 6. 
FTD (2002, 29.07.): ,Middelhoff verlässt Bertelsmann', Nr. 144, S. 1.

FTD (2002, 12.06.): ,Bertelsmann stärkt Position im Musikgeschäft', Nr. 111, S. 3.

GARTLAND, M.P. (2005): Interdisciplinary views of sub-optimal outcomes: Path dependene in the social management sciences, in: The Journal of SocioEconomics, Nr. 34, S. 686-702.

GARUD, R./RAPPA, M. (1994): A socio-cognitive model of technology evolution: the case of cochlear implants, in: Organization Science, Vol. 5, Nr. 3, S. 344-362.

GeOrge, A.L./BENNETT, A. (2005): Case studies and theory development in the social sciences, Cambridge, MA.

GEORGI, D. (2003): Kundenbindungsmanagement im Kundenbeziehungslebenszyklus, in: Bruhn, M./Homburg, C. (Hrsg.): Handbuch Kundenbindungsmanagement, 4. Aufl., Wiesbaden, S. 222-243.

GöÖCK, R. (1968): Bücher für Millionen, Gütersloh.

GoHR, S. (2006): Der Club Bertelsmann: „Mit ganzem Herzen ein DirektmarketingUnternehmen“, in: Direkt Marketing, 42. Jahrgang; Nr. 8, S. 32-35.

GoMm, R. ET AL. (2000): Case study and generalization, in: Gomm, R. et al. (Hrsg.): Case study method, London u.a., S. 98-115.

GranT, R.M. (2002): Contemporary strategy analysis. Concepts, techniques, applications, 4. Aufl., Oxford u. a.

Grant, R.M./NIPPA, M. (2006): Strategisches Management. Analyse, Entwicklung und Implementierung von Unternehmensstrategien, 5. Aufl., München.

GRESOV, C. ET AL. (1993): Organizational design, inertia and the dynamics of competitive response, in: Organization Science, Vol. 4, Nr. 2, S. 181-208.

GuinNANE, T. ET AL. (HRSG.) (2003): History matters: Essays on ecnomic growth, technology, and demographic change, Stanford.

GUJ.DE (2008): ,Gruner+Jahr, Portraits', online, URL: http://www.guj.de/index2.php4, [05.01.2008].

HaLl, D.J./SAIAS, M.A. (1980): Strategy follows structure!, in: Strategic Management Journal, Vol. 1, S. 149-163.

HAMEL, G. (2000): Leading the revolution, Boston.

HAMEL, J. (1992): Introduction: New theoretical and methodological issues, in: Hamel, J. (Hrsg.): Current Sociology, The case method in sociology, Vol. 40, Nr. 1, Bristol, S. 1-7. 
Hammersley, M./Gomm, R. (2000): 'Introduction', in: Gomm, R. et al. (Hrsg.): Case study method, London u.a., S. 1-16.

HANDELSBlatT (2006, 26.09.): ,Der schwarze Panther aus Gütersloh', Nr. 186, S. 10.

HaNDElSBlaTt (2006, 18./19./20.08.): ,Hugendubel bläst zum Angriff, Nr. 159, S. 11.

HANDELSBLATT (2005, 22.03.): ,Fast schon ein Auslaufmodell', Nr. 57, S. 21.

HANDELSBLATT (2004, 16.12.): ,Bertelsmann greift beim Buchclub durch', Nr. 245, S. 14.

HANDElSblatt $(2003$, 18.09.): ,Buchclub in neuem Glanz', Nr. 180, S. 17.

HandelsblatT (2003, 10.03.): ,Das Ende der Beschaulichkeit', Nr. 193, S. 2.

HANDELSBLATT $(2002,12.12$.$) : ,Last chance, last dance', Nr. 240, S. 11$

HANDELSBLATT (1998, 24.03.): ,Bertelsmann stärkt US-Buchgeschäft', Nr. 58.

HANDELSBLATT (1995, 25.11.): ,Bücher bringen die höchste Rendite’, Nr. 228, S. 15.

HANDELSBLATT (1995, 23.06.): ,Vorstandschef Marc Wössner beklagt unbefriedigende Geschäftslage im Inland. Das Buchclub-Geschäft ist ausgereizt.“, Nr. 119, S. 12.

HANDELSBLATT (1992, 02.11.): ,Umorganisation beim Buch und beim Entertainment', Nr. 212, S. 18.

HANDELSBlATT (1988, 29.02.): ,Kooperation mit Quelle verlängert. Clubgeschäft verspricht keine weitere Expansion.', Nr. 41, S. 25.

HANDELSBLATT (1983, 12.12.): ,Fingerübung eines Meisterschülers', Nr. 238.

HANDELSBLATT (1982, 07.12.): ,Bei Umsatzrückgang hierzulande brachten die Auslandstöchter weiteres Wachstum', Nr. 234.

HANDElsblatT (1981, 04.06.): ,Programm reduziert', Nr. 106.

HANNAN, M.T. ET AL. (1996): Inertia and change in the early years: Employment relations in young, high technology firms, in: Industrial and Corporate Change, Vol. 5, Nr. 2, S. 503-536.

Hannan, M.T./FreEMAN, J. (1989): Organizational ecology, Cambridge u.a.

HANNAN, M.T./FrEEMAN, J. (1984): Structural inertia and organizational change, in: American Sociological Review, Vol. 49, Nr. 2, S. 149-164.

HarTley, J. (2004): Case study research, in: Cassell, C./Symon, G. (Hrsg.): Essential guide to qualitative methods in organizational research, London u. a., S. 323-333.

HeINOLD, W.E. (2007): Bücher und Buchhändler, 5. Aufl., Frankfurt/M.

HEINOLD, W.E. (1990): Bücher und Buchhändler, 2. Aufl., Frankfurt/M. 
HelfFERICH, C. (2005): Die Qualität qualitativer Daten. Manual für die Durchführung qualitativer Interviews, 2. Aufl., Wiesbaden.

HiLLER, H. (1966): Der Verlagsbuchhandel, in: Hiller, H./Strauß, W. (Hrsg.): Der deutsche Buchhandel. Wesen, Gestalt, Aufgabe, 3. Aufl. Gütersloh, S. 53-162.

HILLER, H./FüSSEL, S. (2002): Wörterbuch des Buches, 6. Aufl., Frankfurt/M.

HöSCHGEN, H. (1992): Grundbegriffe der Betriebswirtschaftslehre, 3. Aufl., Stuttgart.

HOFER, C.W./SCHENDEL, D. (1978): Strategy formulation: Analytical concepts, St. Paul.

HOPF, C. (1991): Qualitative Interviews in der Sozialforschung. Ein Überblick, in: Flick, U. et al. (Hrsg.): Handbuch Qualitative Sozialforschung, Grundlagen, Konzepte, Methoden und Anwendungen, München, S. 177-182.

HORN, J.T. ET AL. (2006): Learning to let go: Making better exit decisions, in: The McKinsey Quarterly, Nr. 2.

HUFF, J.O. ET AL. (1992): Strategic renewal and the interaction of cumulative stress and inertia, in: Strategic Management Journal, Vol. 13, S. 55-75.

HUGENDUBEL.DE (2008A): ,Unternehmen', online, URL: http://www.hugendubel.de/ unternehmen/hugendubel/unternehmen/Default.aspx [10.02.2008].

HUGENDEUBEL.DE (2008B): ,Chronik', online, URL: http://www.hugendubel.de/ unternehmen/hugendubel/chronik/Default.aspx

HULTÉN, S. (2003): Reconstructing the early history of path-dependency theory, in: Backhaus, J.G. (Hrsg.): Evolutionary economic thought, Northampton, S. 114-124.

HutTeR, M./LANGENBUCHER, W.R. (1980): Buchgemeinschaften und Lesekultur. Studie zum Programmangebot von sechs Buchgemeinschaften (1972-1977), Berlin.

INDUSTRIEMAGAZIN (1987/06): ,Bücher, Bits und Bares'.

INDUSTRIEMAGAZIN (1984, 15.10.): ,Der neue Geist von Gütersloh', Nr. 10.

INTERVIEW BE-07175 (2007, 21.09.): Buchmarktexperte, telefonisch, anonymisiertes Transkript.

INTERVIEW BE-07033 (2007, 17.07.): Buchmarktexperte, persönlich, anonymisiertes Transkript.

INTERVIEW BM-08522 (2008, 05.02.): Aktueller Entscheidungsträger des Bertelsmann Buchclubs, persönlich, anonymisiertes Transkript.

INTERVIEW BM-07410 (2007, 02.07.): Aktueller Entscheidungsträger des Bertelsmann Buchclubs, persönlich, anonymisiertes Transkript. 
INTERVIEW BMA-08593 (2008, 14.02.): Aktueller Verpächter des Bertelsmann Buchclubs, telefonisch, anonymisiertes Transkript.

INTERVIEW BME-07969 (2007, 16.04.): Ehemaliger Entscheidungsträger der Bertelsmann AG, telefonisch, anonymisiertes Transkript.

INTERVIEW BME-07963 (2007, 24.04.): Ehemaliger Entscheidungsträger der Bertelsmann Direct Group, telefonisch, anonymisiertes Transkript.

INTERVIEW BME-07356 (2007, 03.09.): Ehemaliger Programmverantwortlicher des Bertelsmann Buchclubs, persönlich, anonymisiertes Transkript.

INTERVIEW BME-07221 (2007, 18.12.): Ehemaliger Programmverantwortlicher des Bertelsmann Buchclubs, telefonisch, anonymisiertes Transkript.

INTERVIEW BME-07010 (2007, 07.03.): Ehemaliger Programmverantwortlicher des Bertelsmann Buchclubs, persönlich, anonymisiertes Transkript.

INTERVIEW BME-06021 (2006, 21.12.): Ehemaliger Strategieverantwortlicher des Bertelsmann Buchclubs, telefonisch, anonymisiertes Transkript.

JANIS, I.L. (1982): Groupthink: Psychological studies of policy decisions and fiascos, 2. Aufl., Boston.

JOHNSON, J. (2002): How conceptual problems migrate: Rational choice, interpretation, and the hazards of pluralism, in: Economics and Philosophy, Nr. 6, S. 207-234.

JunGERMANN, H. (1976): Rationale Entscheidungen, Bern.

JUNIUS, K. (1997): Economies of Scale: A survey of the empirical literature, Kieler Arbeitspapiere des Instituts für Weltwirtschaft an der Universität Kiel, Nr. 813.

Kelly, D./AMBURGEY, T.L. (1991): Organizational inertia and momentum: A dynamic model of strategic change, in: The Academy of Management Journal, Vol. 34, Nr. 3, S. 591-612.

KIMBERY, J.R. (1987): The study of organization: Toward a biographical perspective, in: Lorsch, J.W. (Hrsg.): Handbook of Organizational Behavior, Englewood Cliffs, S. 223-237.

KOCH, J. (2007): Strategic paths and media management. A path dependency analysis of the German newspaper market of high quality journalism, paper submitted at 31. Workshop der Kommission Organisation', 1./2. März 2007, München.

KoCH, J. (2007A): Strategie und Handlungsspielraum: Das Konzept der strategischen Pfade, in: Zeitschrift für Organisation, 76. Jg., Nr. 5, S. 283-291.

KOLLMANNSBERGER, M. (1995): Buchgemeinschaften im deutschen Buchmarkt. Funktionen, Leistungen, Wechselwirkungen, Wiesbaden. 
KoONTZ, H./O'DonNELl, C. (1955): Principles of management: An analysis of managerial functions, New York.

Kreikebaum, H. (1993): Strategische Unternehmensplanung, 5. Aufl., Stuttgart u. a.

KubiceK, H./ThOM, N. (1976): Umsystem, betriebliches, in: Grochla, E./

Wittmann, W. (Hrsg.): Handwörterbuch der Betriebswirtschaft, Stuttgart, Sp. 3977-4017.

LAATZ, W. (1993): Empirische Methoden. Ein Lehrbuch für Sozialwissenschaftler, Thun u.a.

LANGBEIN, H.B. (1989): Die Buchpreisbindung in der EWG, Europäische Hochschulschriften, Reihe 2, Rechtswissenschaft, Bd. 803, Frankfurt/M.

LANGENBUCHER, W.R. (1985): Bertelsmann nach 1945. Ein Kapitel deutscher Verlags- und Unternehmensgeschichte, in: Bertelsmann (Hrsg.): 1835-1985. 150 Jahre Bertelsmann, München, S. 37-58.

LANGENBUCHER, W.R./TRUCHSEß, W.F. (1974): Buchmarkt der neuen Leser. Studien zum Programmangebot der Buchgemeinschaften (1962-1971), Berlin.

LANT, T.K./MILLIKEN, F.J. (1992): The role of managerial learning and interpretation in strategic persistence and reorientation: An empirical exploration, in: Strategic Management Journal, Vol. 13, S. 585-608.

LAWSON, T. (1997): Economics and reality, London.

LEHNING, T. (2004): Das Medienhaus. Geschichte und Gegenwart des BertelsmannKonzerns, München.

LEVITT, B./MARCH, J.G. (1988): Organizational learning, in: Annual Review of Sociology 14, S. 319-340.

LuCIUS, W.D.v. (1995): Probleme des Verlagsgeschäfts, in: Altenhein, H. (Hrsg.): Probleme des Verlagsgeschäfts. Beiträge zur Entwicklung des Literaturmarktes, Wiesbaden, S. 9-19.

LUDWIG, J. (1997): Zur Ökonomie der Medien: Zwischen Marktversagen und Querfinanzierung, Berlin.

Luhmann, N. (1990): Risiko und Gefahr, Aulavorträge Nr. 48, St. Gallen.

Luhmann, N. (1985): Soziale Systeme, 2. Aufl., Frankfurt/M.

MAHONEY, J. (2000): Path dependence in historical sociology. Theory and society, Vol. 29, S. 507-548.

MARCH, J.G. (1991): Exploration and exploitation in organizational learning, in: Organization Science, Vol. 2, Nr. 1, S. 71-87. 
MARCH, J.G./OLSEN, J.P. (1979): Ambiguity and choice in organizations, Bergen.

MARCH, J.G./SimON, H.A. (1958): Organizations, New York u.a.

MAYNTZ, R. (2005): Soziale Mechanismen in der Analyse gesellschaftlicher MakroPhänomene, in: Schimank, U./Gesoff, R. (Hrsg.): Was erklärt die Soziologie? Methoden, Modelle, Perspektiven, S. 204-227.

MAYRING, P. (2003): Qualitative Inhaltsanalyse. Grundlagen und Techniken, 8. Aufl., Weinheim u. a.

MAYRING, P. (1990): Einführung in die qualitative Sozialforschung, München.

MEUSER, M./NAGEL, U. (2005): ExpertInneninterviews - vielfach erprobt, wenig bedacht. Ein Beitrag zur qualitativen Methodendiskussion, in: Bogner, A. et al. (Hrsg.): Das Experteninterview. Theorie, Methode, Anwendung, 2. Aufl., Wiesbaden, S. 71-93.

MEY, G. (2000): Erzählungen in qualitativen Interviews: Konzepte, Probleme, soziale Konstruktion, in: Sozialersinn, Nr. 1, S. 135-151.

MeYer-DoHm, P. (1966): Das Buch - wirtschaftlich gesehen, in: Hiller, H./ Strauß, W. (Hrsg.): Der deutsche Buchhandel. Wesen, Gestalt, Aufgabe, 3. Aufl., Gütersloh, S. 386-402.

MiLler, D. (1994): What happens after success: The perils of excellence, in: Journal of Management Studies, Nr. 31, S. 325-358.

MiLler, D. (1993): The architecture of simplicity, in: Academy of Management Review, Nr. 18, S. 116-138.

Miller, D. (1990): The Icarus Paradox. How exceptional companies bring about their own downfall, New York.

MintzBerg, H. (1977): Patterns in strategy formulation, in: Management Science, Nr. 24, S. 934-148.

MONGE, P.R. (1995): Theoretical and analytical issues in studying organizational processes, in: Huber, G.P./Van de Ven, A.H. (Hrsg.): Longitudinal field research methods. Studying processes of organizational change, London u. a., S. 267-298.

NORTH, D.C. (1990): Institutions, institutional change and economic performance, Cambridge.

O. V. (2007A): Entwurf eines Gesetzes zur Regelung der Preisbindung bei Verlagserzeugnissen, Stand 20. März 2002, online, URL: http://www.bezregduesseldorf.nrw.de/BezRegDdorf/autorenbereich/Vergabekammer/PDF/Begruend ung_Preisbindungsgesetz.pdf [09.12.2007].

O. V. (2003A): Info. Gesetz über die Preisbindung für Bücher (BUCHPRG) vom 2. September 2002, in: WIC, 09/2003. 
Pearce, J.A/Robinson, R.B. (2007): Formulation, implementation and control of competitive strategy, 10. Aufl., New York.

PIERSON, P. (2000): Increasing returns, path dependence, and the study of politics, in: American Political Science Review, Vol. 94, S. 251-267.

PORTER, M.E. (1999): Wettbewerbsvorteile, 5. Aufl., Frankfurt u. a.

Pralahad, C.K./BeTtis, R.A. (1986): The dominant logic. A new linkage between diversity and performance, in: Strategic Management Journal, Vol. 7, S. 458-501.

PRALAhad, C.K /Hamel, G. (1990): The core competence of the corporation, in: Harvard Business Review, Vol. 68, Nr. 3, S. 79-91.

Preußner, N. (1989): Geschäftskonzept des Clubbereichs, in: Bertelsmann (Hrsg.), Meilensteine für die Controlling-Arbeit des Club-Bereichs 1970-1994, Gütersloh, S. 229-248.

RAUTENBERG, U. (2006): Das Buch in der Alltagskultur: Eine Annäherung an zeichenhaften Buchgebrauch und die Medialität des Buches, in: Delp, Ludwig (Hrsg.): Das Buch in der Informationsgesellschaft. Ein buchwissenschaftliches Symposium, Buchwissenschaftliche Forschungen 06/2005, Wiesbaden, S. 7-30.

RÉGNER, P. (2005): Identifying inertia - Processes and practices in the development of strategy lock-in. Paper presented at the ' $21^{\text {st }}$ EGOS colloquium' 2005, Berlin.

RöTTGERS, J. (2003): Mix, Burn \& R.I.P. Das Ende der Musikindustrie, Hannover.

Ross, L. (1977): The intuitive psychologist and his shortcomings. Distortions in the attribution process, in: Berkowitz, L. (Hrsg.): Advances in Experimental Social Psychology, Nr. 10, New York, S. 173-220.

RosZINSKY-TERJUNG, A. (1981): Imperien auf dem Hauptvorschlagsband, in: Arnold, Heinz-Ludwig (Hrsg.): Literaturbetrieb in der Bundesrepublik Deutschland. Ein kritisches Handbuch, 2. Aufl., München, S. 112-124.

Rohloff, A. (2003): UFA - eine starke Marke im Wandel der Zeiten, Potsdam.

RTL-TELEVISION.DE (2008): ,Chronik', online, URL: http://www.rtltelevision.de/chronik.html [07.01.2008].

RÜHLE, G. (1985): Die Büchermacher. Von Autoren, Verlegern, Buchhändlern, Messen und Konzernen, Frankfurt/M.

RÜRUP, B. ET AL. (1997): Ökonomische Analyse der Buchpreisbindung, Frankfurt/M.

SCHAPER, F.-W. (1977): Handbuch des Buchhandels, Sonderdruck, Band IV, Übrige Formen des Bucheinzelhandels - Zwischenbuchhandel und Buchgemeinschaft, Wiesbaden u. a. 
SCHMALEN, H. (1996): Grundlagen und Probleme der Betriebswirtschaft, 10. Aufl., Köln.

SCHMIDT, L. (2006): Technologie als Prozess. Eine empirische Untersuchung organisatorischer Technologiegestaltung am Beispiel von Unternehmenssoftware, Online-Dissertation, abrufbar über URL: http://www.diss.fuberlin.de/2006/346/index.html, [04.03.2008].

SCHÖNSTEDT, E. (1991): Der Buchverlag. Geschichte, Aufbau, Wirtschaftsprinzipien, Kalkulation und Marketing, Stuttgart.

SCHREYÖGG, G./KLIESCH-EBERL, M. (2007): How dynamic can organizational capabilities be? Towards a dual-process model of capability dynamization, in: Strategic Management Journal, Vol. 28, S. 913-933.

SCHREYÖGG, G. (2006): Organisation. Grundlagen moderner Organisationsgestaltung, 4. Aufl., Wiesbaden.

SCHREYÖGG, G. ET AL. (2003): Organisatorische Pfade - Von der Pfadabhängigkeit zur Pfadkreation?, in: Schreyögg, G./Sydow, J. (Hrsg.): Managementforschung 13, Strategische Prozesse und Pfade, S. 257-294.

SCHREYÖGG, G. (1993): Umfeld der Unternehmung, in: Wittmann, W. et al. (Hrsg.): Handwörterbuch der Betriebswirtschaft, Teilband 3, 5. Aufl., Sp. 4232-4248.

SCHREYÖGG, G. (1991): Der Managementprozess - neu gesehen, in: Staehle, W.H./Sydow, J. (Hrsg.): Managementforschung 1, Berlin u. a., S. 255-289.

SCHREYÖGG, G. (1984): Unternehmensstrategie: Grundfragen einer Theorie strategischer Unternehmensführung, Berlin u. a.

SCHULER, T. (2005): Die Mohns, Frankfurt/M.

SCHUlze, B. ET AL. (2005): The Bertelsmann AG: An exploratory case study on synergy management in a globally acting media organization, in: The International Journal of Media Management, Vol. 7, Nr. 3/4, S. 138-147.

SChumanN, M./Hess, T. (2006): Grundfragen der Medienwirtschaft, 3. Aufl., Berlin u. a.

SCHumpeTER, J.A. (1939): Business cycles, 1. Aufl., New York.

SiEBEN, G./SCHILDBACH, T. (1994): Betriebswirtschaftliche Entscheidungstheorie, 4. Aufl., Düsseldorf.

SIGGELKOW, N. (2007): Persuasion with case studies, in: Academy of Management Journal, Vol. 50, Nr. 1, S. 20-24.

SKINNER, B.F. (1938): The behavior of organisms, New York. 
SNOW, C.C./HAMBRICK, D.C. (1980): Measuring organizational strategies: Some theoretical and methodological problems, in: The Academy of Management Review, Vol. 5, Nr. 4, S. 527-538.

StAeHLe, W.H. (1991): Management. Eine verhaltenswissenschaftliche Perspektive, 6. Aufl., München.

STAW, B.M. (1981): The escalation of commitment to a course of action, in: Academy of Management Review, Vol. 6, Nr. 4, S. 577-587.

STAW, B.M. (1976): Knee-deep in the big muddy: A study of escalating commitment to a chosen course of action, in: Organizational Behavior and Human Performance, Vol. 16, S. 27-44.

STEINMANN, H./SCHREYÖGG, G. (2005): Management. Grundlagen der Unternehmensführung, 6. Aufl., Wiesbaden.

STIEHL, U. (1993): Der Verlagsbuchhändler. Ein Lehr- und Nachschlagewerk, Stuttgart.

STIGLER, G.J./BECKER, S.B. (1977): De gustibus non est disuptandum, in: The American Economic Review, Vol. 67, Nr. 2, S. 76-90.

STINCHCOMBE, A.L. (1965): Social structure and organizations, in: March, J.G. (Hrsg.): Handbook of Organizations, Chicago, S. 142-193.

STOCK, M. (1992): Der neue Rundfunkstaatsvertrag, in: Rundfunk und Fernsehen, 40. Jhg., Nr. 2, S. 189-221.

STRAUß, W. (1974): Bemerkungen zur gesellschaftlichen Relevanz von Buchgemeinschaften, in: Swierk, A. (Hrsg.): Beiträge zur Geschichte des Buches und seiner Funktion in der Gesellschaft. Festschrift für Hans Widmann zum 65. Geburtstag, Stuttgart, S. 280-290.

STRAUß, W. (1966): Die deutschen Buchgemeinschaften, in: Hiller, H./Strauß, W. (Hrsg.): Der deutsche Buchhandel. Wesen, Gestalt, Aufgabe, 3. Aufl. Gütersloh, S. 266-278.

SUDMAN, S./BLAIR, E. (1998): Marketing research. A problem solving approach, Boston, u. a.

SUßMANN, W. (1967): Der Wettbewerb im westdeutschen Bucheinzelhandel, Berichte des Instituts für Buchmarkt-Forschung, Nr. 35/36, Hamburg.

SYDOW, J. ET AL. (2005): Organizational paths: Path dependency and beyond, paper presented at the '2 2 st EGOS colloquium' 2005, Berlin.

SZ (2006, 26.09.): ,Aktion Sorgenkind', Nr. 222, S. 17.

SZ (1989, 05.12.): ,Bertelsmann hofft auf ,Marketing-Mix', Nr. 279.

SZ (1989, 23.05.): ,Klubs bringen Bertelsmann 3 Milliarden DM ein’, Nr. 16. 
SZ (1988, 21.10.): ,In der Vielfalt liegt die Kraft', Nr. 227.

SZ (1984, 25.06.): ,Neue Ufa-Mediengesellschaft', Nr. 144.

SZ (1982, 07.12.): ,Bertelsmann strafft weiter das Bücherangebot', Nr. 281.

TEECE, D.J. ET AL. (1997): Dynamic capabilities and strategic management, in: Strategic Management Journal 18, S. 509-533.

TeECE, D.J. (1988): Technological change and the nature of the firm, in:

Dosi, G. et al. (Hrsg.): Technological change and economic theory, London, S. 256-281.

Thalia.DE (2008): ,Geschichte des Unternehmens', online, URL: http://www. service.thalia.de/index.php?content=geschichte\&chapter=holding, [10.02.2008].

THELEN, K. (1999): Historical institutionalism in comparative politics, in: Annual Review of Political Science, S. 369-404.

TIETZ, B. (1985): Königsidee Buchgemeinschaft. Neue Leser durch eine neue Marktpolitik, in: Bertelsmann (Hrsg.): 1835-1985. 150 Jahre Bertelsmann, München, S. 339-356.

TIETZEL, M. (1995): Literaturökonomik, Tübingen.

Tripsas, M./GaVETTI, G. (2000): Capabilities, cognition, and inertia: Evidence from digital printing, in: Strategic Management Journal, Nr. 21, S. 1147-1161.

UFA (2007): Träume Bilder - Bilder Träume, UFA Film \& Produktion GmbH (Hrsg.), Berlin.

UlRICH, P./FLuRI, E. (1995): Management, 7. Auflage, Bern u. a.

UlRICH, H. (1970): Die Unternehmung als produktives soziales System, 2. Aufl., Bern/Stuttgart.

VAUS, DE DAVID (2003): Research design in social research, London u. a.

WALSH, J.P. (1988): Selectivity and selective perception: An investigation of managers' belief structures, in: Academy of Management Journal, Vol. 31, Nr. 4, S. 873-896.

WathieU, L. (2004): Conusmer habituation, in: Management Science, Vol. 50, Nr. 5, S. 587-596.

WeICK, K.E./BougON, M. (1986): Organizations as cognitive maps. Charting ways to success failure, in: Sims, jr. H.P./Gioia, D.A. et al. (Hrsg.): The thinking organization, San Francisco, S. 102-135.

WeLTBILD.COM (2008A): ,Über uns', online, URL: http://www.weltbild.com/110ueberuns.html [10.02.2008]. 
WeLTBILD.COM (2008B): ,Chronik', online, URL: http://www.weltbild.com/170chronik.html [10.02.2008].

WIC (2006/01): ,Menschen für Bücher begeistern', BUA-UP2/82, Interview mit Anita Offel-Grohmann, [24.08.2006].

WIC (2005/06): ,Editorial', BUA-UP2/82, Carro, F. (Verf.), [24.08.2006].

WIC (2005/04): ,Neues Club-Finanz-Magazin ,Club Extra Geld \& Mehr', BUAUP2/82, o. V., [28.08.2006].

WIC (2005/04A): ,Junge Entdecker im Visier', BUA-UP2/82, o. V., [29.08.2006].

WIC (2005/04B): ,Viel in Bewegung', BUA-UP2/82, o. V. [24.08.2006].

WIC (2005/03): ,Für eine Club-Mitgliedschaft begeistern', BUA-UP2/82, o. V. [24.08.2006].

WIC (2005/02): ,Das Buch im Fokus', BUA-UP2/82, o. V., [28.08.2006].

WIC (2005/02): ,Editorial', BUA-UP2/82, Sommer, M.-O. (Verf.), [24.08.2006].

WIC (2004/10): ,Weihnachtsworte des Geschäftsführers', BUA-UP2/82, Sommer, M.-O. (Verf.), [29.08.2006].

WIC (2004/07): ,Editorial', BUA-UP2/82, Wittenberg, R. (Verf.), [29.08.2006].

WIC (2004/07): ,Guter Kompromiss. Club und Börsenverein verständigen sich auf neues Potsdamer Protokoll', o. V., BUA-UP2/82, [29.08.2006].

WIC (2004/03): ,Neues Konzept zur stationären Mitgliederwerbung', BUA-UP2/82, o. V., [25.08.2006].

WIC (2004/01): ,Schnäppchen für alle: Neues Angebot für lesefreudige Sparfüchse', BUA-UP2/82, o. V., [24.08.2006].

WIC (2004/01A): ,Das Image des Clubs weiter aufladen!', BUA-UP2/82, Interview mit Mathias Winter, [24.08.2006].

WIC (2003/09): ,Suche nach einem fairen Kompromiss', BUA-UP2/82, Interview mit Peter Schaper, [24.08.2006].

WIC (2003/09): ,Info: Gesetz über die Preisbindung für Bücher (BUCHPRG) vom 2. September 2002', BUA-UP2/82, o. V., [25.08.2006].

WIC (2003/07): ,Editorial', BUA-UP2/82, Wittenberg, R. (Verf.), [24.08.2006].

WIC (2003/03): ,Der Club als gern gesehener Partner', BUA-UP2/82, o. V., [29.08.2006].

WIC (2003/03A): ,Das volle Programm', BUA-UP2/82, o. V., [24.08.2006]. 
WIC (2003/02): , Rainer Wittenberg im Interview mit der „Wir im Club“", BUAUP2/82, [30.08.2006].

WIC (2002/08): ,Lagebericht des Vorstandsvorsitzenden Gunter Thielen: ,Die Bertelsmann AG steht zum Club!“, BUA-UP2/82, [30.08.2006].

WIC (2002/04): ,Vorwort', BUA-UP2/82, Böttger, W. (Verf.), [23.08.2006].

WIC (2001/05): Vorwort der Geschäftsführung, BUA-UP2/82, Böttger, W. (Verf.), [24.08.2006].

WIC (2001/04): , "Operation Sonnenblume“. Der Spezialclub als Ergänzung zum universalen Vater', BUA-UP2/82, o. V. [23.08.2006].

WIC (2001/03): ,Pressekonferenz: Den Club neu erleben!', BUA-UP2/82, o. V., [24.08.2006].

WIC (2001/02-03): ,Ansprache', BUA-UP2/82, Böttger, W. (Verf.), [24.08.2006].

WIC (1998/02): ,Club Premiere', BUA-UP2/82, o. V., [23.08.2006].

WIC (1997/11): ,Es geht voran', BUA-UP2/82, o. V., [30.08.2006].

WIC (1997/11): ,Editorial', BUA-UP2/82, Refäuter, D. (Verf.), [28.08.2006].

WIC (1997/02): ,Eröffnung des Buchclubs China', BUA-UP2/82, o. V., [28.08.2006].

WIC (1996/11): ,Bücherwürmer, Autoren und Wohlfühlatmosphäre', BUA-UP2/82, o. V., [28.08.2006].

WIC (1996/08): ,Best!Seller', BUA-UP2/82, o. V., [24.08.2006].

WIC (1996/01): ,Reihenweise gute Bücher', BUA-UP2/82, o. V., [24.08.2006].

WIC (1995/11): ,"Flöti“" spielt die erste Geige', BUA-UP2/82, o. V., [25.08.2006].

WIC (1995/10): ,Ansprache', BUA-UP2/82, Greiner, G. (Verf.), [25.08.2006].

WIC (1994/06): ,40 Jahre VG Rheda', BUA-UP2/82, o. V., [29.08.2006].

WIC (1994/01): ,Editorial', BUA-UP2/82, Krümmer, S. (Verf.), [29.08.2006].

WIC (1992/06): ,Der Bücherbund wird wieder profitabel', BUA-UP2/82, Schäfer, T. (Verf.), [24.08.2006].

WIC (1992/02): ,Unser Club übernimmt Deutschen Bücherbund', BUA-UP2/82, o. V., [25.08.2006].

WIC (1991/07-08): ,Aktuelles', BUA-UP2/82, Wössner, F. (Verf.), [25.08.2006].

WIC (1991/04): ,Das Geschäft boomt: Der Club-Foto-Dienst', BUA-UP2/82, o. V., [25.08.2006].

WIC (1991/02): ,Spielzeug ist kein Kinderspiel', BUA-UP2/82, o. V., [29.08.2006]. 
WIC (1990/05): ,Der Buchmarkt - ein Buch mit 7 Siegeln?', BUA-UP2-82, o. V., [29.08.2006].

WIC (1989/04): ,Deutsche Buchgemeinschaft', BUA-UP2/82, o. V., [29.08.2006].

WIC (1988/08): ohne Titel, BUA-UP2/82, o. V., [24.09.2006].

WIC (1988/01): ,Der internationale Konzern-Führungskreis', BUA-UP2/82, o. V., [24.08.2006].

WIC (1987/06-07): ,Unsere Spezialclubs', BUA-UP2/82, o. V., [24.08.2006].

WIC (1986/11): ,Funktionsorganigramm des deutschen Clubgeschäfts', BUAUP2/82, o. V., [24.08.2006].

WILlKE, H. (1996): Dimensionen des Wissensmanagements - zum Zusammenhang von gesellschaftlicher und organisatorischer Wissensbasierung, in: Schreyögg, G./ Conrad, P. (Hrsg.): Managementforschung 6: Wissensmanagement, Berlin u. a., S. 263-304.

WIRTSCHAFTSWOCHE (1998, 29.10.): ,Alte Grenzen überwinden', Nr. 45.

WiRTSCHAFTSWOCHE (1985, 14.06.): ,Aus Fehlern gelernt', Nr. 25.

WiTTE, E. (1993): Entscheidungsprozesse, in: Wittmann, W. et al. (Hrsg.):

Handwörterbuch der Betriebswirtschaft, Teilband 1, 5. Aufl., Stuttgart, S. 910-920.

WiTTE, E. (1992): Entscheidungsprozesse, in: Frese, E. (Hrsg): Handwörterbuch der Organisation, 3. Aufl., Stuttgart, S. 552-565.

WiTZEL, A. (2000): Das problemzentrierte Interview, in: Forum Qualitative Sozialforschung, Online-Journal, Nr. 1, abrufbar über URL: http://qualitativeresearch.net/jqs, [04.03.2008].

WÖHE, G. (2002): Einführung in die allgemeine Betriebswirtschaftslehre, 21. Aufl., München.

WygODA, S. (2005): Risiko als Chance, Berlin.

W\&V (2005, 15.09.): ,Ein Koffer nach Berlin', Nr. 37, S. 30.

W\&V (2005, 28.04.): ,Kunden verzweifelt gesucht', Nr. 17, S. 10.

YIN, R.K. (1994): Case study research. Design and methods, 2. Aufl., Thousand Oaks u. a. 


\section{Anhang 1: Stichwortkataloge}

Abbildung 23: Stichwortkataloge als Leitfaden während der Archivrecherche

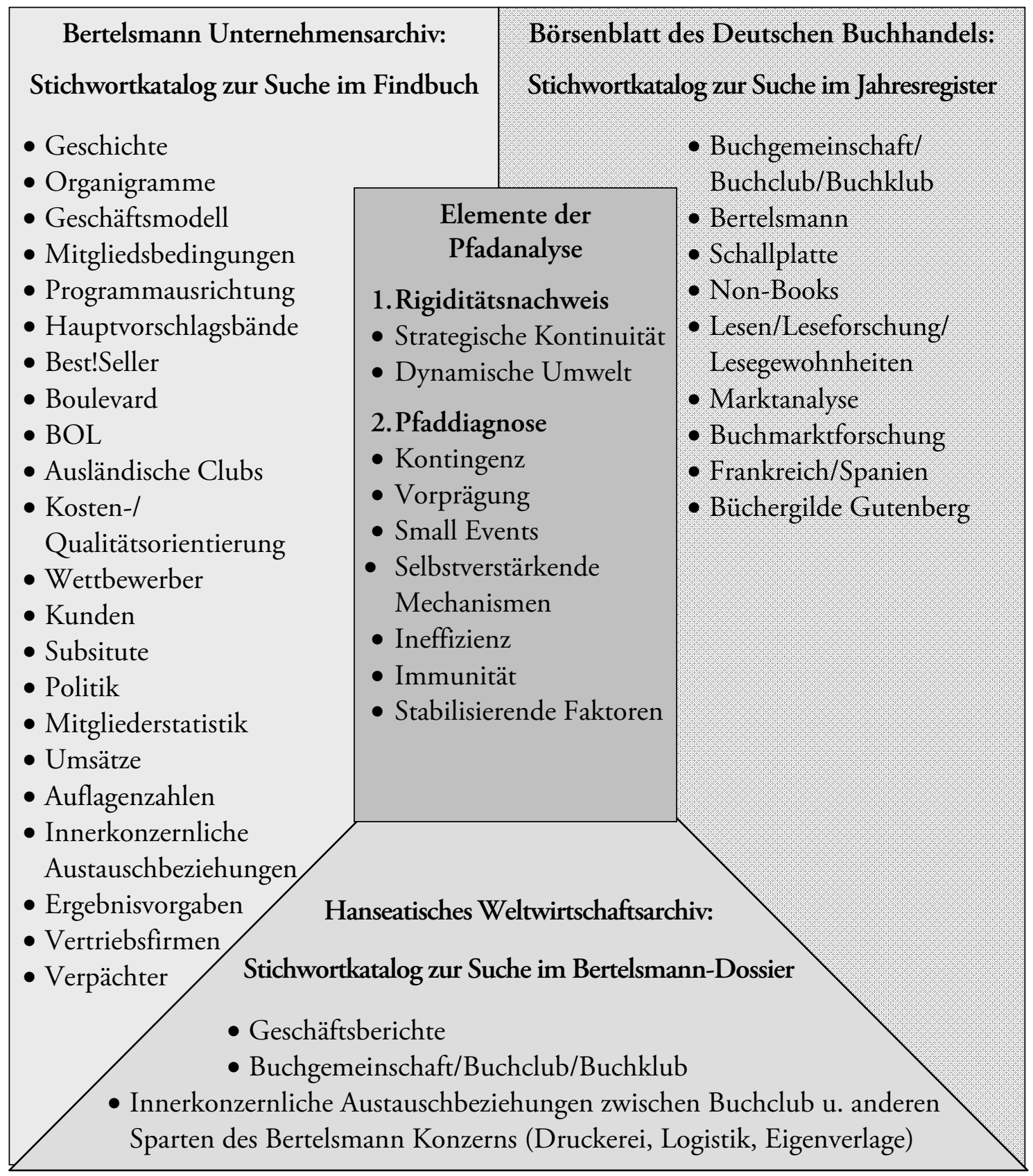

Quelle: Eigene Darstellung. 



\section{Anhang 2: Beispiel eines Interviewleitfadens}

\section{Interviewleitfaden für Interview BME-07963}

Zeit:

$$
\text { 24.04.2007, } 60 \text { Min. }
$$

Interviewer: Philip Holtmann, FU Berlin

Interviewter: Ehemaliger Entscheidungsträger der Bertelsmann Direct Group

Art: $\quad$ telefonisch

\section{Informationen zur Person}
A Position im Unternehmen (In- und Ausland)
B Zugehörigkeit zum Unternehmen
- Welche Stelle hatten Sie während Ihrer Tätigkeit bei Bertelsmann wie lange?
C Aufgabengebiete
- Was waren Ihre Aufgaben in den einzelnen Positionen?

\section{Strategische Ausrichtung des Clubs}

\section{A Definition des Geschäfts}

- Beschreiben Sie das Geschäftsmodell des Bertelsmann Buchclubs in Deutschland!

- Wie lässt sich das Geschäftskonzept definieren?

- Haben sich die strategischen Ziele verlagert? (Preis, Qualität, Auswahl, Mitgliedschaftsverpflichtung)?

- Wenn Sie sich einmal das Geschäftsmodell zur Gründungszeit ansehen und den Buchclub von heute - welche wesentlichen Unterschiede sehen Sie?

B Wirtschaftliche Situation des Buchclubs

- Wie beurteilen Sie die wirtschaftliche Situation des Buchclubs?

- Seit Anfang der 70er liest man immer wieder von der Krise des Buchclubs. Wie schätzen Sie die Situation ein?

C Kontinuität vs. Wandel des Geschäftsmodells

- Wie flexibel ist die strategische Ausrichtung des Buchclubs?

- Ausgehend von der Gründung in der Nachkriegszeit bis heute hat sich der Buchmarkt auf Angebots- wie auf Nachfrageseite stark gewandelt.

Wie hat Bertelsmann darauf reagiert?

- Wird das bestehende Modell hinterfragt und diskutiert? 
- Welche Versuche wurden unternommen, den Club zukunftsfähig zu gestalten?

- Wie beurteilen Sie deren Erfolg? Wie konsequent wurden die Alternativen durchgeführt?

\section{Mögliche Einflussfaktoren auf strategische Entscheidungen}

- Wie werden strategische Entscheidungen im Buchclub gefällt?

- Wer fällt sie? Wer hat noch Einfluss darauf?

- Wie groß ist der Spielraum für die verantwortlichen Geschäftsführer bzw. Programmverantwortlichen?

- Gibt es ein Beispiel von strategischen „Experimenten“, die ausprobiert, jedoch nicht konsequent weiterverfolgt wurden?

- Wie stark wirken die Profitabilitätvorgaben des Managements auf die Flexibilität für strategische Entscheidungen?

- Welche Rolle spielt der Buchclub für die anderen Sparten des Gesamtkonzerns Bertelsmann?

- Gibt es Kuppelprodukte/Verbundvorteile/Synergieeffekte?

- Gibt es Querfinanzierungen durch den Buchclub? (Hintergrund: bspw. zwischen dem Buchclub und eigenen Verlagen bzgl. Lizenzvergaben, Rechte der Autoren)

- Welche weiteren Konzernsparten profitieren von den Aktivitäten des Buchclubs?

- Wie hoch ist die persönliche (emotionale) Bindung an den Club (bspw. durch die Eigentümerfamilie)?

- Wie stark beeinflusst das Image des Buchclubs bei Kunden die strategische Handlungsfähigkeit des Buchclubs? Welche Rolle spielen die Kundenerwartungen bei der strategischen Ausrichtung des Clubs?

- Nach welchen Kriterien wird die Personalauswahl vorgenommen?

\section{E Ausländische Bertelsmann Buchclubs (Frankreich / Spanien)}

- Werden strategische Entscheidungen für die ausländischen Bertelsmann Buchclubs von Deutschland aus gefällt oder agieren die ausländischen Clubs autonom?

- Wie bezeichnen Sie das Geschäftsmodell von France Loisirs/Circulo de Lectores?

- Was ist das Kerngeschäftsfeld?

- Welche wesentlichen Unterschiede bestehen zum deutschen Club?

- Was macht France Loisirs Ihrer Meinung nach besser oder schlechter?

- Kann der deutsche Club von France Loisirs lernen und erfolgreiche Strategien übernehmen?

\section{F Abschlussfragen}

- Können Sie mir weitere Kontaktpersonen nennen, die für meine Thematik interessant wären?

- Darf ich Ihnen - sofern bei der Auswertung des Interviews noch Fragen auftreten sollten - Anmerkungen bzw. Fragen per Email zusenden? 


\section{Anhang 3: Chronologie des Bertelsmann Buchclubs Deutschland}

Tabelle 3: Historische Entwicklung des Bertelsmann Buchclubs

\begin{tabular}{lll}
\hline Jahr & Ereignis & Quelle \\
\hline 1950 & $\begin{array}{l}\text { Gründung der Buchgemeinschaft ,Lesering Das Ber- } \\
\text { telsmann Buch }\end{array}$ & BI (1960/09) \\
\hline 1950 & Gründung des Schloß Verlags & Tietz, B. (1985), S. 346 \\
\hline 1951 & Einführung der Freundschaftswerbung & $\begin{array}{l}\text { BUA-0006/15 (Bertelsmann- } \\
\text { Clubarbeit: Chronik, 1977) }\end{array}$ \\
\hline 1952 & Aufbau der Lexikonredaktion & Bertelsmann (1985), S. 403. \\
\hline 1952 & Einführung der Buswerbung & Gööck, R. (1968), S. 131 \\
\hline 1953 & Umbenennung in ,Bertelsmann Lesering' & BUA (2006). \\
\hline 1953 & Einführung der Treuebände & BUA-0006/15 (Bertelsmann- \\
\hline 1954 & Gründung Verlagsgemeinschaft Rheda (VR) & Clubarbeit: Chronik, 1977) \\
\hline 1955 & Gründung der ,Bertelsmann Lesering GmbH' & BUA (2006) \\
\hline
\end{tabular}

1955 Erweiterung des Druckereibetriebes Mohn \& Co. Gööck, R. (1968), S. 154 $\mathrm{GmbH}$

1955 Gründung des Versandhauses Heim \& Buch

BUA-0006/15 (Bertelsmann-

Clubarbeit: Chronik, 1977)

1955 Gründung des Jugend-Leserings BUA-0006/15 (Bertelsmann-

Clubarbeit: Chronik, 1977)

1956 Umbenennung der ,Bertelsmann Lesering GmbH’ in BUA (2006) ,Bertelsmann GmbH'

1956 Auflösung des Versandhauses Heim und Buch $\quad$ BI (1956/05)

1956 Gründung der Vertriebsgemeinschaft Buch und Wissen, BI (1960/09) Rheda

1956 Gründung des Schallplattenrings $\quad$ BI (1960/01)

1956 Gründung des Kartographischen Instituts Bertelsmann BUA-0006/15 (Bertelsmann-

Clubarbeit: Chronik, 1977) 


\begin{tabular}{lll}
1958 & Gründung von Sonopress GmbH & BI (1960/09) \\
\hline 1958 & Gründung der Ariola GmbH & BI (1960/09) \\
\hline 1959 & Neugliederung des Verlagsbereichs & Bertelsmann (1985), S. 403 \\
\hline 1959 & Erwerb des Mosaik Verlages & Bertelsmann (1985), S. 403 \\
\hline 1959 & Gründung des Instituts für Buchmarktforschung & Bertelsmann (1985), S. 403 \\
\hline 1959 & Gründung des Kommissionshaus Buch und Ton & $\begin{array}{l}\text { BI (1959/04); BI (1959/07); } \\
\text { Adron, L. (1983) }\end{array}$ \\
\hline
\end{tabular}

1961 Gründung des Europarings der Buch- und Schallplat- $\quad$ BI (1961/09), S. 7; tenfreunde BI (1962/01), S. 3

1962 Gründung der Firma Vergara Circulo de Lectores S.A. BUA-0006/15 (BertelsmannClubarbeit: Chronik, 1977)

1962 Ausdehnung der Clubarbeit auf Schweiz und Österreich BUA-UP3/44-7 (Lesefamilie, 1988)

1963 Übernahme des Rings der Musikfreunde (P.P. Kelen) BUA-0006/15 (BertelsmannClubarbeit: Chronik, 1977)

1963 Übernahme des Europäischen Buchclubs und des Euro- BUA-0006/15 (Bertelsmannpäischen Phonoclubs Clubarbeit: Chronik, 1977); Adron, L. (1983)

1964 Start des Club-Reisedienstes für Lesering-Mitglieder Adron, L. (1983)

1964 Eröffnung der ersten Bertelsmann-Bücherstube in Kiel BUA-0006/15 (BertelsmannClubarbeit: Chronik, 1977)

1965 Jugend-Lesering wird in Bertelsmann Lesering überführt Gööck, R. (1968), S. 204

1965 Gründung des Christlichen Bildungskreis Gööck, R. (1968), S. 202

1965 Gründung des Leserings mit dem großen Schallplatten- BUA-0006/15 (Bertelsmannprogramm Clubarbeit: Chronik, 1977)

196640 \% Beteiligung an der österreichischen Buchgemein- BUA-0006/15 (Bertelsmannschaft Donauland Clubarbeit: Chronik, 1977); BI (1966/03), S. 2 f.

1966 Eröffnung des ersten Partner-Club-Centers BUA-0006/15 (BertelsmannClubarbeit: Chronik, 1977)

1966 Gründung der ,Reinhard Mohn oHG’ als Nachfolge- Gööck, R. (1968), S. 211 firma der ,Bertelsmann GmbH' 
1967 Gründung des Europaclub voor boeken en grammofoonplaten N.V., Holland
BUA-0006/15 (BertelsmannClubarbeit: Chronik, 1977);

Gööck, R. (1968), S. 212

1967 Übernahme des Reise- und Verkehrsverlags

Gööck, R. (1968), S. 214

1967 Gründung des Central-Buchversand

Gööck, R. (1968), S. 216

1968 Gründung der Verlagsgruppe Bertelsmann

BI (1969/01), S. 6;

BI $(1968 / 01)$, S. 7

\begin{tabular}{lll}
1968 & Gründung des Bertelsmann Jugendbuchverlag & BI (1969/03), S. 8 \\
\hline 1968 & Gründung des Bertelsmann Lexikon-Verlags & BI (1969/02), S. 4 \\
\hline 1968 & Gründung des Bertelsmann Sachbuchverlags & BI (1969/04), S. 2 \\
\hline 1969 & Umbenennung der Bücherstuben in Club-Center & Buchmarkt (1978/11), S. 42 \\
\hline 1969 & Deutscher Bildungskreis & $\begin{array}{l}\text { BUA-0006/15 (Bertelsmann- } \\
\text { Clubarbeit: Chronik, 1977) }\end{array}$ \\
\hline
\end{tabular}

1969 Gründung der ersten Buchclubs in Lateinamerika

Bertelsmann (1985), S. 404

1970 France Loisirs in Frankreich

BUA-UP3/44-7 (Lesefamilie, 1988)

197050 \% Beteiligung an der Deutschen Buchgemeinschaft (DBG)

BUA-0006/464 (Deutsche BuchGemeinschaft, 1970)

1971 Umbenennung des, Kommissionshaus Buch und Ton' Adron, L. (1983) in 'Vereinigte Verlagsauslieferung' (VVA)

1971 Beginn der Clubarbeit in Südamerika und Portugal BUA-UP3/44-7 (Lesefamilie, 1988)

1971 Umwandlung in Bertelsmann AG BUA-0006/15 (BertelsmannClubarbeit: Chronik, 1977)

1972 Erstangebot von Spielen als Programm 0006/465 (Hennig, 1977), S. 5

1973 100\% Übernahme der Europäischen Bildungsgemein- Roszinsky-Terjung, A. (1981), schaft (EBG)

S. $112-124$

1973 Gründung "Club top 13": Neuer Club für Schlagerfans

BUA-0006/465 (Hennig, 1977),

S. 5

1974 Erstangebot von Foto-Kameras

BUA-0006/15 (Bertelsmann-

Clubarbeit: Chronik, 1977)

1975 Erstangebot von kunstgewerblichen Erzeugnissen in limitierten Auflagen

BUA-0006/465 (Hennig, 1977),

S. 5 
1975 Euroclub in Italien

1975 Erstangebot eines Fotolabor-Service
BUA-UP3/44-7 (Lesefamilie, 1988); SZ (1989, 23.05.)

BUA-0006/465 (Henning, 1977),

S. 5
Bertelsmann (1985), S. 46

BUA-0006/465 (Henning, 1977), S. 5

BUA-UP3/44-7 (Lesefamilie, 1988)

1977 Errichtung der Bertelsmann Stiftung

BUA-0006/15 (Bertelsmann-

Clubarbeit: Chronik, 1977)

Börsenblatt (1983, 08.03.), S. 539;

FAZ (1983, 04.04.)

Ladenketten (Deutschland)

Bertelsmann (1985), S. 56

1984 Gründung des Geschäftsbereichs Fernsehen

WIC (1988/01), S. 2f.;

1986 Übernahme des US-Verlags Doubleday;

Übernahme der Doubleday-Buchlubs in USA, England, BUA-UP3/44-7 (Lesefamilie, 1988)

Kanada, Australien und Neuseeland

1989100 \% Übernahme der Deutschen Buch-Gemeinschaft WIC (1989/04) (DBG)

1989 Schließung der Buchclubs in Südamerika Die Welt $(1989,20.05$.

1989 Kooperation mit VISA $\quad$ FAZ $(1989,21.07$.

1991 24,9 \% Beteiligung am Deutschen Bücherbund (DBB) WIC (1992/02), S. 3

1992100 \% Übernahme des Deutschen Bücherbunds (DBB) WIC (1992/06), S. 2;

WIC (1992/02), S. 3

1993 Gründung der Buch AG BG (1993/94), S. 4-7

1993 Einführung der Club Premieren WIC (1998/02), S. 4

1996 Start der Medienversandfirma Best!Seller $\quad$ WIC (1996/08)

1996 Eröffnung erste Filiale der Boulevard-Buchhandlung in WIC (1996/11) Gelsenkirchen

1997 Eröffnung des Buchclubs China in Shanghai WIC (1997/02)

1997 Repositionierung des Clubs: Werbekampagne, Branding, WIC (1997/11); WIC (1998/02) Katalogformat, Buch als Schwerpunkt, Exklusivität 


\begin{tabular}{lll}
1999 & Best!Seller wird eingestellt & BuchMarkt (1999/09) \\
\hline 2000 & Gründung der Direct Group & BG (1999/2000), S. 33 \\
\hline 2001 & Start des Internet-Auftritts: www.derclub.de & WIC (2001/03) \\
\hline 2001 & Testclub Moments & WIC (2002/04), S. 3. \\
\hline 2001 & Integration von BOL in die Buchclubs in Europa und Asien & WIC (2001/05), S. 1 \\
\hline 2002 & Eröffnung der Boulevard-Buchhandlung Bielefeld & BuchMarkt (2002, 13.02.) \\
\hline 2002 & Einstellung des Spezialclubs ,Moments' & Buchreport (2002, 18.12.), S. 8 \\
\hline 2002 & Trennung von BOL & WIC (2002/08) \\
\hline 2003 & Verkauf der Boulevard-Buchhandlung, Bielefeld & BuchMarkt (2003, 05.02.) \\
\hline 2003 & $\begin{array}{l}\text { Test des Club-TV-Magazins 'Leben und Genießen' } \\
\text { (Filialmagazin) }\end{array}$ & WIC (2003/03a), S. 5 \\
\hline
\end{tabular}

2003 Bisher größte Medienkampagne (TV-Spots, Anzeigen) WIC (2004/01a), S. 5

2004 Test von Club-Centern in Warenhäusern Buchreport $(2005,13.10$.)

2004 Testlauf des neuen Best!Seller-Katalogs (Modernes An- WIC (2004/01), S. 2; tiquariat) BuchMarkt (2004/04)

\begin{tabular}{lll}
\hline 2004 & Einführung von SmartShops (Promotion Shops) & WIC (2004/03); WIC (2005/03) \\
\hline 2005 & Einführung der Preis- und Qualitätsgarantie & WIC (2005/03), S. 4 \\
\hline
\end{tabular}

2005 Erste Ausgabe des Club Finanz-Magazins, Club Extra WIC (2005/04), S. 2; Geld \& Mehr' Gohr, S. (2006), S. 32-35

2005 Test Mini-Shops WIC (2005/04b), S. 9

2005 Test Integration von Cafes in Buchclub-Ladenfläche WIC (2005/04b), S. 9

2005 Start von, Columbus' für Kinder und Jugendliche WIC (2005/04a), S. 4

2006 Strategische Partnerschaft mit CreditPlus-Bank Gohr, S. (2006), S. 32-35

2006 Erste Ausgabe des Familienmagazins, Club Extra Family' Gohr, S. (2006), S. 32-35

2006 Kooperation mit AXA Konzern zum Vertrieb von Versi- Gohr, S. (2006), S. 32-35 cherungen

2006 Erste Ausgabe des Supplements, Club Extra 50 aktiv' Gohr, S. (2006), S. 32-35

2006 Kooperation mit DiViDi (Online DVD-Verleih) Gohr, S. (2006), S. 32-35;

Buchreport $(2006,27.04$.) 


\title{
K Ö L N E R W I S S E N S C H A F T S V E R L A G
}

\author{
Wirtschaftswissenschaften: Themenbereich Unternehmensführung
}

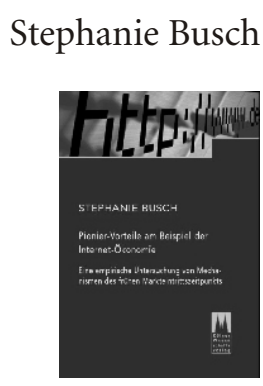

Oliver Reichel

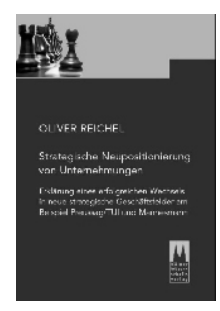

Sascha Albers

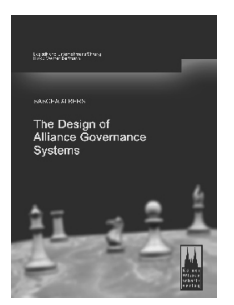

Tobias Braun

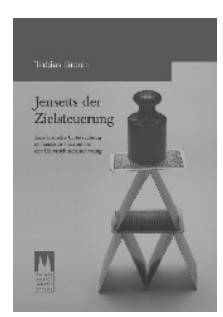

\author{
Pionier-Vorteile am Beispiel der Internet-Ökonomie
}

Nachdem Ende der 9oer Jahre die Vorteilhaftigkeit eines frühen Markteintritts und damit die Wirkung von Pionier-Vorteilen proklamiert wurden, widersprachen die ab dem Jahr 2000 auftretenden Probleme in Internet-Unternehmungen dieser Ansicht. Aufgrund offensichtlicher Pionier-Nachteile stellt sich die Frage, ob vorsichtiges Abwarten und Lernen aus den Fehlern des Pioniers die aussichtsreichere Markteintrittsstrategie in neuen Märkten darstellen kann.

Das vorliegende Werk von Stephanie Busch erweitert den bisher unzureichenden Erkenntnisstand zu Pionier-Vorteilen in der wissenschaftlichen Diskussion. Ferner beinhaltet es die empirische Überprüfung von Pionier-Vorteilen mit Hilfe qualitativer Fallstudienuntersuchungen von sechs deutschen Startup-Unternehmungen in der Internet-Ökonomie.

2005, Paperback, 346 Seiten, Preis 29,90 Euro; ISBN 3-937404-13-9

Strategische Neupositionierung von Unternehmungen

Der Wandel vom traditionellen Industriekonzern zum Touristikdienstleister oder zum Telekommunikationsanbieter stellt einen fundamentalen (und seltenen) Einschnitt in die Entwicklung einer Unternehmung dar. Die Fälle Preussag/TUI und Mannesmann zeigen jedoch, dass das Phänomen der "Strategischen Neupositionierung" (SNP) in der Praxis durchaus relevant ist. Oliver Reichel untersucht Faktoren, die zum Erfolg eines Wechsels in neue, unverbundene Geschäftsfelder bei Aufgabe aller bisherigen Aktivitäten beitragen. Dabei wird der ressourcenbasierte Ansatz in Verbindung mit dynamischen Fähigkeiten herangezogen, ergänzt durch eine explorative Untersuchung. Dabei zeigt der Autor neben den erfolgsrelevanten Faktoren auch Motive für eine SNP sowie deren Ablauf und Realisierungswege auf.

2005, Paperback, 379 Seiten, Preis 34,80 Euro; ISBN 3-937404-17-1

\section{The Design of Alliance Governance Systems}

Strategic alliances have emerged as an important element of firms' strategies. Following suit, research on alliances has blossomed. However, most contributions which address the alliance governance problem are yet rather vague and selective in their conception of alliance governance structures as well as the factors which influence their suitability. The aim of this book is to provide recommendations on the problem of alliance governance design. Following the configurational approach, Sascha Albers develops a comprehensive model of alliance governance systems.

Band 3 der Reihe "Logistik und Unternehmensführung",

hrsg. v. Prof. Dr. Dr. h.c. Werner Delfmann

2005, Hardcover, 350 Seiten, Preis 59,9o Euro; ISBN 3-937404-16-3

Jenseits der Zielsteuerung - Eine kritische Untersuchung zielbasierter Instrumente der Unternehmenssteuerung

Balanced Scorecard, Management by Objectives, Kennzahlensteuerung, Hoshin Kanri - die Idee einer Unternehmenssteuerung durch Ziele existiert in vielen Varianten. Gemeinsam ist ihnen eine auffällige Diskrepanz zwischen versprochenen und eingelösten Steuerungserfolgen: Trotz verheißungsvoller Versprechungen ist der Praxiseinsatz dieser Instrumente vor allem durch Probleme und Mißerfolg geprägt. Tobias Braun zeigt, daß es die Steuerungslogik der Zielsteuerung selbst ist, die für das Versagen in der Praxis verantwortlich ist. Auf Basis psychologischer und systemtheoretischer Erkenntnisse werden der Steuerungsanspruch der Zielsteuerung und die Verwendung des Konstrukts "Ziel" sowohl auf der Ebene der Mitarbeiterführung als auch auf der Ebene der Unternehmensführung untersucht und zentrale Mängel identifiziert.

2004, Hardcover, 432 Seiten, Preis 54,80 Euro; ISBN 3-937404-06-6 